\title{
Report to the American Physical Society by the study group on light-water reactor safety*
}

The issue of light-water reactor (LWR) safety has been the subject of a part-time, year-long study sponsored by the American Physical Society. The goal of the study was the assessment of some of the technical aspects of the safety of large light-water nuclear power reactors typical of present commercial practice in the Unted States. The report examines issues related to safe operation of LWRs; the research and development program responsible for establishing and enhancing safety; and the consequences of accidents for public health and welfare. The report in no way deals with the need for nuclear power or its benefits, and should not be considered as a net assessment of the risks versus the benefits of nuclear reactors. Since the risks of ecological impacts of other energy technologies are not addressed, no recommendations are made concerning the specific reactor program which should be followed in the immediate future. Among the areas covered in the report are primary pressure-vessel integrity; quality assurance; accident initiation from operator error, transients, and sabotage; the adequacy of present emergency core-cooling system designs; the calculation of long-term consequences to health of one particular low-probability accidental release of radioactivity; and the experimental and calculational (computer-code-development) aspects of the present reactor safety research program. A number of recommendations are contained with the report, mainly addressed to ways in which the safety of the present LWRs can be improved or better understood.

\section{APS Study Group Participants}

H. W. Lewis, Chairman

University of California, Santa Barbara, California 93106

R. J. Budnitz

Lawrence Berkeley Laboratory, University of California, Berkeley, California 94720

A. W. Castleman

Brookhaven National Laboratory, Upton, Long Island 11973

D. E. Dorfan

University of California, Santa Cruz, California 95064

F. C. Finlayson

Aerospace Corporation, Los Angeles, California 90009

R. L. Garwin

IBM Thomas J. Watson Research Center, Yorktown Heights, New York 10598

L. C. Hebel

Xerox Corporation, Rochester, New York 14644

S. M. Keeny, Jr.

The Mitre Corporation, McLean, Virginia 22101

R. A. Muller

University of California, Berkeley, California 94720

T. B. Taylor

International Research and Technology Corporation, Arlington, Virginia 22209

\section{G. F. Smoot}

University of California, Berkeley, California 94720

F. von Hippel

Princeton University, Princeton, New Jersey 08540

APS Council Review Committee

Hans Bethe

Cornell University, Ithaca, New York 14850

W. K. H. Panofsky

Stanford Linear Accelerator Center, Stanford University, Stanford, California 94305

V. F. Weisskopf

Massachusetts Institute of Technology, Cambridge, Massachusetts 02139

(Received 28 April 1975)

\footnotetext{
*Supported by the National Science Foundation and the Atomic Energy Commission. Contract EN-43893.
} 
Rev. Mod. Phys., Vol. 47, Suppl. No. 1, Summer 1975 


\section{CONTENTS}

List of Abbreviations Used in the Text ............ S4

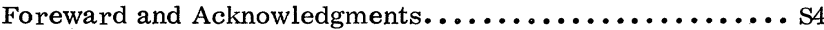

I. Summary of Conclusions and Major Recommendations.. S5

A. Safety through careful design, construction, and operation

B. Primary engineered safety features

C. Accident containment and consequences

D. Major recommendations

II. Introduction. ......................... S9

A. Coverage of report $\quad$ S9

B. The nature of the hazard $\quad$ S9

$\begin{array}{ll}\text { C. The nature of safety } & \text { S9 }\end{array}$

D. Evaluating the safety of complex systems $\quad \mathrm{S10}$

E. The role of licensing, regulation, and research in safety

F. Organization of the report

III. Primer on Light-Water Nuclear Reactors and Related Safety Issues............................. 13

A. Nuclear reactor physics $\quad \mathrm{S} 13$

B. Description of a commercial light-water reactor S15

C. Safety considerations $\quad \mathrm{S} 22$

D. The roles of various organizations involved in assuring safety in the nuclear power industry $\quad$ S23

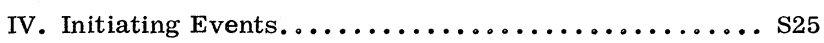

A. Primary system integrity $\quad$ S25

$\begin{array}{ll}\text { B. Transients } & \mathrm{S} 27\end{array}$

C. Quality assurance $\quad \mathrm{S} 28$

D. Operator error $\quad \mathbf{S 3 0}$

E. Sabotage $\quad$ S31

V. Course of an Accident........................... 33

A. Description of a loss of coolant accident (LOCA) S33

B. ECCS analysis and evaluation methods-Acceptance Criteria

C. ECCS evaluation

D. Containment behavior in a LOCA

E. The release of radionuclides and consequences of a core melt

F. Synopsis of the reference accident consequences due to ${ }^{137} \mathrm{Cs}$ alone

G. Other radiation doses from radionuclides released to the atmosphere

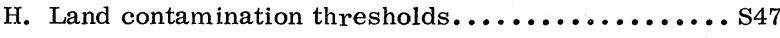

I. Comparison with analysis in Draft WASH-1400 of reference accident (PWR2) consequences

J. Mitigating the consequences of a large release of radioactivity

VI. The Light-Water Reactor Safety Research Program. . S53

A. Introduction $\mathbf{S 5 3}$

B. Program resources and emphases $\quad$ S54

C. Research to improve primary system integrity $\quad$ S54

D. Separate effects tests . $\$ 55$

E. Power Burst Facility (PBF) program $\quad 559$

F. LOFT and Semiscale integral test experimental programs

G. Containment research

H. Research on radiological consequences, accident $\begin{array}{ll}\text { mitigation, and recovery } & \mathrm{S} 71\end{array}$

$\begin{array}{ll}\text { I. Research techniques } & \mathrm{S} 72\end{array}$

J. Overall evaluation of the light-water reactor safety program

K. Organization and management of reactor safety research

Appendix I: The Loss-of-Coolant Accident. ........... S81

Appendix I. A: Generalized description of loss-ofcoolant accident for PWRs and BWRs S81

Appendix I. B: Loss-of-coolant accident without effective emergency core cooling S84

Appendix I. C: Relationship of LOCA phenomena to the ECCS Acceptance Criteria $\quad$ S85

Appendix II: Containment and Consequences. ...........S95

A. Radioactive release from core $\quad \mathbf{S 9 5}$

B. Atmospheric dispersion $\quad$ S96

$\begin{array}{ll}\text { C. Dose effect } & \mathbf{S 1 0 0}\end{array}$

D. Land and property contamination $\quad \mathrm{S102}$

E. Consequence calculations $\quad \mathrm{S103}$

F. Mitigating the consequences $\quad \mathrm{S109}$

G. Improved containment systems $\quad \mathrm{S110}$

H. Siting policies $\quad$ S111

Appendix III: Description of the ECCS Acceptance

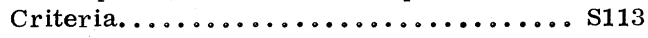

References $\ldots \ldots \ldots \ldots \ldots \ldots \ldots \ldots \ldots \ldots \ldots \ldots \ldots \ldots \ldots$. 121 


\section{LIST OF ABBREVIATIONS USED IN THE TEXT}

\begin{tabular}{|c|c|}
\hline $\mathrm{AC}$ & $\begin{array}{l}\text { Acceptance Criteria (promulgated by the AEC } \\
\text { for ECCS designs) }\end{array}$ \\
\hline $\mathrm{AEC}$ & U. S. Atomic Energy Commission \\
\hline APS & American Physical Society \\
\hline ASME & American Society of Mechanical Engineers \\
\hline BWR & Boiling-Water Reactor \\
\hline $\mathrm{CHF}$ & Critical Heat Flux \\
\hline DBA & Design Basis Accident \\
\hline DNB & Departure from Nucleate Boiling \\
\hline $\mathrm{ECC}$ & Emergency Core Coolant \\
\hline ECCS & Emergency Core Cooling System \\
\hline EPA & U. S. Environmental Protection Agency \\
\hline EPRI & Electric Power Research Institute \\
\hline ERDA & $\begin{array}{l}\text { U. S. Energy Research and Development } \\
\text { Administration }\end{array}$ \\
\hline FY & Fiscal Year \\
\hline GI Tract & Gastro-Intestinal Tract \\
\hline ICRP & $\begin{array}{l}\text { International Commission on Radiological } \\
\text { Protection }\end{array}$ \\
\hline INEL & Idaho National Engineering Laboratory \\
\hline LOCA & Loss-of-Coolant Accident \\
\hline LOFT & Loss-of-Fluid Test Facility \\
\hline LPCI & Low-Pressure Coolant Injection System \\
\hline LWR & Light-Water Reactor \\
\hline NAS & National Academy of Sciences \\
\hline $\mathrm{NRC}$ & U. S. Nuclear Regulatory Commission \\
\hline NSF & National Science Foundation \\
\hline NSSS & Nuclear Steam Supply Systems \\
\hline PAHR & Post-Accident Heat Removal System \\
\hline PBF & Power Burst Facility \\
\hline PWR & Pressurized-Water Reactor \\
\hline QA & Quality Assurance \\
\hline REG & AEC Directorate of Regulation \\
\hline RSR & AEC Division of Reactor Safety Research \\
\hline SCRAM & $\begin{array}{l}\text { Emergency shut-down system for terminating } \\
\text { the reactor chain reaction }\end{array}$ \\
\hline S.T.P. & Standard temperature and pressure \\
\hline
\end{tabular}

\section{FOREWORD AND ACKNOWLEDGMENTS}

The American Physical Society has engaged over the past few years in activities beyond those traditional for the Society. In 1973 the Society explored mechanisms by which it could contribute to the alleviation of the energy crisis. In addition to other activities, it was decided to sponsor a study of reactor safety, an important subject with substantial scientific and technological content. This is the report of that year-long study by a dozen part-time participants with various levels of prior experience in the reactor field. The group met in Los Alamos during the month of August, 1974, and also had approximately a dozen two-day meetings, many phone calls, and much correspondence.

The group is grateful to all the genuine experts who gave liberally of their time in educating us about this intricate subject. In turn, we hope that our report will help inform the scientific and technical community about some of the technical issues of reactor safety.

We particularly acknowledge the contributions of S. Johnson, G. Brockett, and P. Davis, who served as consultants and who provided continuing support to our study. Their patient exposition of the fine points of reactor design, operation, and regulation was invaluable.
We also particularly acknowledge the contributions of D. H. Coward, who helped organize the group, and of H. A. Bethe, W. K. H. Panofsky, and V. F. Weisskopf, who served as the APS Council Review Committee.

The cooperation of the reactor community and of experts in related fields such as biological effects of radiation was outstanding, and we acknowledge the help of the many representatives of the vendors, reactor designers, safety analysts, and others who provided us with information. In particular, members of the staffs of the $\mathrm{AEC}^{1}$ Division of Reactor Safety Research and of the AEC Office of Regulation gave willingly of their time and resources; the individuals involved are too numerous to mention by name. Also, the group is grateful for briefings and cooperation from all four American vendors of light-water reactors: Babcock \& Wilcox, Combustion Engineering, General Electric, and Westinghouse. It is fair to say that we do not feel that we have been excluded from any information necessary to our task. $^{2}$

We were fortunate that early in our study we were given copies in preliminary draft of the AEC-sponsored study of reactor safety (chaired by Professor Norman Rasmussen of M.I.T., and known as WASH-1400). WASH-1400 is a detailed event-tree and fault-tree analysis of light-water reactor accident sequences. Its purpose was to make a quantitative estimate of the likelihood of accident consequences of a given severity. We did not undertake a review of that study as such, although it will be mentioned frequently in our report.

The gracious hospitality of the Los Alamos Scientific Laboratory and the administrative assistance of the staff of the American Physical Society are also acknowledged. This study was supported by the National Science Foundation and the U. S. Atomic Energy Commission.

The study participants have all agreed on both the broad conclusions and the more detailed individual recommendations contained in the body of the report. We believe this is significant in view of the diverse backgrounds of the group. Our individual technical expertise ranges widely, covering theoretical and experimental physics, chemistry, and engineering. While a few of the group had some background in reactor safety, the majority of the group had not previously considered these issues. Some of the group had participated in previous technical assessments of broad national issues; for sev-

\footnotetext{
${ }^{1}$ Since this work was begun the Atomic Energy Commission has been split in two parts: the Energy Research and Development Administration (ERDA) and the Nuclear Regulatory Commission (NRC), and the exact distribution between ERDA and $\mathrm{NRC}$ of the responsibility that formerly resided in the AEC is still not clear. For this reason we have referred throughout the report to the AEC with the understanding that the reader will interpret all forward-looking references to the AEC as really directed to the relevant components of ERDA or NRC.

${ }^{2}$ There is one exception. Early in our study we became interested in the safety record (in particular primary system integrity) of naval reactors, which, though smaller, have accumulated more reactor years of service than have the civilian reactors of comparable design. We made a major effort to obtain sufficient information, with due regard for questions of classification and national security, to help us in our study. We were refused any access by Admiral H. G. Rickover.
} 
eral others, this study was a first experience. We are pleased with the degree of consensus that we have achieved, albeit regretful that more time was not available for further investigation of some of the important issues involved.

\section{SUMMARY OF CONCLUSIONS AND MAJOR RECOMMENDATIONS}

A central issue in the operation of light-water reactors is the prevention of a major release and widespread dispersal of radioactivity, which could have serious consequences to the public. The safety record of light-water reactors to date has been excellent, in that there has been no major release of radioactivity. These reactors have been designed with numerous safety features, engineered to prevent foreseeable accidents. These safety features are backed up by other safety features intended to prevent major release of radioactivity in the event of an accident. Moreover, very conscientious efforts have been made in developing the procedures and practices involved in licensing, quality assurance, operation, and inspection of these reactors to insure sound construction and operation within specified safety limits.

In the course of this study, we have not uncovered reasons for substantial short-range concern regarding risk of accidents in light-water reactors. While at present a complete quantitative assessment of all important aspects of reactor safety and behavior under unusual circumstances cannot be made, we are confident that a much better quantitative evaluation and consequent improvements of the safety situation can be achieved over the next decade if certain aspects of the safety research program are substantially improved and the results of the research are implemented. Because of the serious potential consequences of a major release of radioactivity, and in view of existing safety-related technological opportunities, we believe that there should be a continuing major effort to improve light-water reactor safety as well as to understand and mitigate the consequences of possible accidents. Our recommendations are directed towards these objectives.

\section{A. Safety through careful design, construction, and operation}

The safety philosophy of the nuclear industry has emphasized design which can provide tolerance against malfunctions. This approach has laid a good foundation for reactor safety, and it has resulted in reactors designed, constructed, and operated for safety, not only under normal operating conditions but also in a wide range of abnormal circumstances. A great deal of research, development, and quality control has gone into guaranteeing the integrity of the fuel elements and cladding, the integrity of the enclosing primary system, the general structural soundness of the entire reactor, and the ability to control the reactor under both normal and abnormal conditions.

Although we have not been able to analyze all of the many possible failure sequences for light-water reactors, one which we have studied in detail is the possible failure of the integrity of the primary reactor pressure vessel. We find that reactor vessels are constructed of materials chosen with care and are designed with substantial safety factors. The reactor vessel is subject to careful scrutiny and testing. Based on our study, we believe that catastrophic rupture of the primary pressure vessel is not likely to be an important contributor to accident initiation; however, this is dependent upon maintaining a strong quality assurance program.

Primary system piping is also subject to careful scrutiny and testing. The well-known cases of cracks in pipes and failures of valves in reactor operation, on the one hand, reflect deficiencies in fabrication or design; but, on the other hand, they demonstrate the success of the overall safety system and procedures which identified their existence early enough to prevent more serious consequences. Continued open discussion and analysis of such failures can lead to improvements in safety and can provide the data base for a more accurate estimate of the probability of more serious incidents. These defects underline the ongoing need for the nuclear industry and the regulatory bodies to continue improvement of inspection and test techniques. It is important that licensing and regulation be conducted in such a way as to continue to ensure openness in the quality assurance program and to provide better-quantified evaluation of the success of the program. We also note that each year human error on the part of reactor operators seems to initiate or aggravate at least a few incidents of potential safety significance. In fact, unless diligence is maintained, quality assurance and human error may well represent a limiting factor in maintaining safe operation.

It is difficult to quantify accurately the probability that any accident-initiating event might occur. Many aspects need to be better understood through experience and research before such calculations are tractable. Although the probabilities of major accidents seem small, their quantification deserves more attention within the reactor safety community than it has received up to now. We did not have the resources to carry out an independent evaluation of this aspect of the recent AEC Reactor Safety Study (Draft WASH-1400), but we recognize that the event-tree and fault-tree approach can have merit in highlighting relative strengths and weaknesses of reactor systems, particularly through comparison of different sequences of reactor behavior. However, based on our experience with problems of this nature involving very low probabilities, we do not now have confidence in the presently calculated absolute values of the probabilities of the various branches.

We have reservations about the present almost exclusive emphasis in the licensing process on the "design basis accident" concept in which certain highly stylized accidents are used as yardsticks against which the performance of various systems is evaluated. While we agree that analysis of such accidents is an important check upon the general safety of reactor designs, we are concerned that other types of possible accidents may consequently receive insufficient attention in design, construction, licensing, and operation. 


\section{B. Primary engineered safety features}

In our study we centered much attention on the "engineered safety features." Because these features are not used in normal operation but are specifically intended to prevent an abnormal incident from becoming an accident, there is only limited operating experience with them. In addition, because of the complexity of the phenomena involved, these features are very difficult to simulate on a computer or to test in simulated accident conditions.

Therefore, there is a lack of well-quantified understanding of the performance of some of these special systems under some severe accident conditions.

One of the most important of the engineered safety features is the fast-acting $\mathrm{SCRAM}^{3}$ system for shutting down the chain reaction in the event of an emergency. Certain transients which are anticipated to occur from time to time (pressure, temperature, reactivity) might play an important role in accident initiation. It is very important to shut down the chain reaction during a large transient. While the SCRAM designs, as now prescribed, seem to us to be highly reliable, not enough is known about the effects of transients in the extremely unlikely event that the reactor does not SCRAM. We believe that insufficient attention has been given to the analysis of transients, although it is encouraging that these areas are now being given intensive study. In addition, we are concerned about transient behavior which might occur simultaneously with a massive electrical failure. While there are redundant off-site power sources, the emergency on-site (diesel) power sources are a recognized weak point.

The emergency core cooling system (ECCS) is the engineered safety feature that has received the most publicity, attention, and research. The ECCS is intended to provide emergency cooling to present the reactor fuel from melting or losing structural integrity in the event there is a loss of primary system fluid.

We have no reason to doubt that the ECCS will function as designed under most circumstances requiring its use. However, no comprehensive, thoroughly quantitative basis now exists for evaluating ECCS performance, because of inadequacies in the present data base and calculational codes. In addition, it is not clear that the present approximate calculations, even though based on generally conservative detailed assumptions, will in all cases yield conservative assessments of ECCS performance.

We have examined the AEC reactor safety research program intended to resolve these uncertainties. Expanded experimental tests and advanced calculational code development are now under way, with the goal of accomplishing a sufficient quantitative comparison between calculation and experiment so that the technical community can reach consensus on ECCS effectiveness. That consensus can only be reached through several years of effort, using improved research techniques, and with

\footnotetext{
${ }^{3} \mathrm{SCRAM}$ is not in fact an acronym. Although its precise origins are shrouded in mystery, it seems to have come into use as a term for emergency shutdown procedure around 1942, at the first reactor built by the University of Chicago. As the name implies, the reactor personnel who shut down the chain reaction were also to get out as quickly as possible.
}

more open publication and review of the results. We doubt that a complete quantitative evaluation of ECCS effectiveness can be achieved through the present program. We recommend below several possible approaches for improvement.

\section{Accident containment and consequences}

The last line of defense in preventing or mitigating the release of radioactivity is a further set of engineered safety features designed as a backstop in case of significant failure of the safety features discussed above. The greater part of this last safety umbrella is the containment machinery and building which enclose the entire reactor primary system. These containments, which have worked well in controlling routine and minor radioactive emissions, have not yet been subjected to test by a large-scale controlled or accidental release. More research toward increasing the effectiveness of containment devices would be prudent, along with more vigorous pursuit of the possibilities for major improvements in containment design.

Although a major release of radioactivity is unlikely, it is important to calculate the types and extent of consequences of releases under various circumstances. We have found that these calculations are very difficult. There are significant uncertainties in nearly every category of potential consequences: immediate deaths, latent cancers, and property damage/denial. We have made no independent studies of acute effects, the estimates of which are particularly dependent upon details of local siting, weather, and population, and upon important uncertainties in acute biological effects of radiation. However, for the same releases and the same basic references for the biological effects as taken in Draft WASH-1400, we estimate substantially larger long-term consequences, particularly concerning land damage/denial and possible latent cancers from exposures to individuals who live in areas which are contaminated below the evacuation thresholds used in Draft WASH-1400. ${ }^{4}$ The social significance of the long-term consequences depends in part upon the probability of the assumed release, regarding which we have made no independent assessment. However, the uncertainties in estimates of consequences need to be resolved because they have important implications in reactor design, siting policy, and protection against potential sabotage. In analyzing the societal risk-benefit balance of commercial nuclear reactors, one must be able to estimate with reasonable confidence both the probability and consequences of system failure; research must continue on both.

Considering the great social importance of reactor safety and the large present and future capital investment in light-water reactors, the current funding of safety research is relatively small. We believe that the many technological opportunities for the enhancement of reactor safety warrant the investment of additional funds in safety research.

${ }^{4} \mathrm{We}$ understand that substantial revisions are being considered before publication of the final WASH-1400 report (private communication, NRC, 17 March 1975). 


\section{Major recommendations}

Many recommendations are made in the body of this Report. A few of the major ones are summarized here, but in each case the reader is referred to the main text for detailed discussions of the background and rationale. Our major recommendations, which have not been ranked according to their importance, include the following:

(1) Human engineering of reactor controls, which might significantly reduce the chance of operator errors, should be improved. We also encourage the automation of more control functions and increased operator training with simulators, especially in accident-simulation mode.

(2) Measures should be taken to quantify the effectiveness of the present quality assurance program, using both the analysis of experience already reported and new measurements on the quality assurance system.

(3) The techniques used in Draft WASH -1400 for the calculation of accident sequences and their probabilities should be:

- employed to estimate quantitatively whether assumed subsystem failure data are compatible with the observed individual small accidents;

- used to provide parametric studies of the effects of phenomena which are ill-understood in the identified sequences;

- refined so that they can be used for continuing risk assessment on a routine basis with a growing data base of failure data.

(4) The Draft WASH-1400 analysis of accident consequences should be redone taking into account the modifications discussed in our report, in order to obtain corrected consequence estimates. The results will help to determine the magnitude of the benefits which might be obtained from the introductions of design changes and means of mitigation of accident consequences.

(5) The problem of sabotage and its effect on increasing the risk of radioactivity release should be studied carefully. We have no way of estimating the present likelihood of sabotage; however, we believe that reactor security can be improved and have specific recommendations for studies that go beyond those already underway.
(6) The ECCS safety margin should be quantified, and if necessary, improved through one or more of the following approaches:

- the substitution of more easily analyzable or more effective ECCS concepts;

- a much stronger theoretical and calculational development effort combined with a much improved experimental program, the results of which must be published openly for evaluation by the technical community;

- a series of large-scale experiments along with some standardization of reactors. Detailed planning and analysis for this approach should begin immediately in case it should be decided in the future that it is needed. There should be increased emphasis on realistic calculations and experiments as opposed to those which merely attempt to set upper limits on the behavior of a reactor in an accident. In view of the number of reactors now operating and being planned, we believe it is important that the reactor safety research program quickly take major steps to bring about a convincing resolution of the uncertainties in EECS performance.

(7) In the area of safety research, more emphasis should be placed on seeking improvements in containment methods and technology. In particular, controlled venting of the containment building in case of overpressure should be studied. A careful assessment should also be made of the benefits and costs of alternative siting policies, such as remote, underground, and nuclear-park siting.

(8) There should be more effort to resolve major uncertainties in estimating consequences, including improvement of the biological-effects data base. Techniques for mitigation of consequences should be developed, especially in connection with the problems of decontamination after a large accident.

(9) While we strongly endorse the substantial improvements that have been made in the safety research programs and in the openness to scrutiny by the technical public in the last two years, additional measures should be taken to continue to improve the research program and techniques and to assure that the results of both experimental and computer code development work related to safety are openly published. 


\section{INTRODUCTION}

\section{A. Coverage of report}

This report examines the safety issues related to the accidental release of radioactivity from commercial light-water reactors. The original charter of the group, "Reactor Safety," was so broad that we decided to confine our attention to light-water-moderated reactors, which are presently the reactor type used almost exclusively for commercial electrical service in the United States. Even "light-water-reactor safety" was too broad for the modest effort we were able to mount. We have therefore tried to isolate some of the critical technical matters and have studied the safety research and development program. On these restricted subjects, we have endeavored to provide a technical analysis in this report.

We did not study the issue of plant effluents in normal operation or other aspects of the nuclear fuel cycle, such as reprocessing and transport of radioactive materials and storage of radioactive wastes; nor did we study the question of the protection of fissionable materials from theft or misuse. We also made no study of any other reactor types, such as high-temperature gas-cooled reactors or liquid-metal fast-breeder reactors. We felt that as members of the technical community, we could make our most significant contribution by investigating the technical issues involved in the safe operation of light-water reactors and by evaluating the research and development program concerning these issues. We hope, through the medium of the report, to provide both the American Physical Society membership and the larger technical community with a technical foundation on the safety of light-water reactors and a review of the current safety research program.

\section{B. The nature of the hazard}

The important potential hazard in a reactor incident is the release of radioactivity, not energy. It is physically impossible for a light-water reactor to explode in the same fashion as a nuclear weapon (as explained in Sec. III). However, it is physically possible for large amounts of radioactive materials, accumulated within the reactor core, to be released into the environment. Even after the power-producing chain reaction is shut down, fissionproduct decay still generates heat at an initial rate of about $225 \mathrm{MWt}$ in a $1000 \mathrm{MWe}$ reactor. ${ }^{5}$ If a loss-ofcoolant accident should occur, this residual heat production would be sufficient to melt the core and release a large amount of radioactivity. Because such accidents could conceivably result in the release of large amounts of radioactivity, much design and construction effort has been devoted to reducing the probability of such an event and to limiting the escape of radioactivity to the environment.

\footnotetext{
${ }^{5} \mathrm{MWt}$ is the rate of production of thermal energy (heat) in megawatts. MWe is the rate of production of electrical energy; taking into account the efficiency (usually about 1/3) of the reactor-generator system, 1000 MWe corresponds to about 3300 MWt.
}

\section{The nature of safety}

The amount of radioactivity in a reactor is sufficient to cause serious damage if released to the environment. Like other systems, natural or man made, one cannot expect reactors to be "perfectly safe," but rather one must decide whether the overall risks to society from accidental release are sufficiently small so that reactors are "safe enough." Defining what is "safe enough" is clearly a very difficult problem.

In practice, decisions on what is "safe enough" are made almost unconsciously by the public and by various licensing bodies. Despite the lack of a common measure of costs and benefits, all manner of costs are accepted by the general public to achieve perceived benefits (for example, in the United States each year over a thousand people are accidentally electrocuted, yet there is no pressure for the abolition of electricity and not much for improvements which would decrease the electrocution rate). It is even more difficult to define what is "safe enough" in situations involving potentially serious accidents with extremely low probabilities because costs are replaced by potential risks and because there are no agreed figures of merit. Not only do different people place different emphasis on such values as the preservation of life and land, but they even measure benefits and risks in different units.

Another problem which arises in dealing with the spectrum of risks (potential costs) of a reactor accident is that a dichotomy syndrome sometimes manifests itself. The spectrum of possible accidents may become characterized by the "maximum credible accident," which will/ will not happen. Similarly, the performance of safety systems in a multitude of situations is sometimes simplified to "the emergency system will/will not work" or even "reactors are/are not safe." In assessing safety, one must avoid this fallacy of reducing a complicated situation to the simple black-and-white picture of yes/no.

Similarly, there is a natural tendency continually to improve the safety of a system to assure that it is "safe enough." Any system can be made safer, and there is usually some additional cost. It is essential to balance the increased safety against the increased costs. As an example, the recently required new safety features and air pollution control featu res for automobiles have entailed new costs, among them higher car prices, reduced gas mileage and performance, and increased nuisance in use. Some of these new features have been perceived as beneficial and retained, while others, such as the safety belt interlock, have been rejected. For reactors, the two chief costs of increased safety are increased cost of electric power and its possible decreased availability. It is therefore a relevant public concern to determine at what level of safety reactors are "safe enough."

The issues of public concern about the safety of lightwater reactors may then be generalized into three broad questions:

1. Are existing light-water reactors "safe enough?"

2 . Can the safety level of existing reactors be maintained adequately in the future?

3. Will our confidence in the safety of light-water reactors designed and built in the future be greater than 


\section{for today's reactors?}

We have not studied the benefits of nuclear power, much less attempted to weigh them against the risks; therefore, we cannot answer these three broad questions. However, it is helpful to keep them in mind while approaching the technical issues.

\section{Evaluating the safety of complex systems}

One approach to designing safety into large systems (and reactors are typical examples) is by making the systems sufficiently redundant and diverse that several relatively independent failures are necessary to generate a serious accident sequence. The low accident probabilities for reactors rest on the multiplication of probabilities of several independent events. However, such small probabilities are often overshadowed by socalled "common-mode" failures, which are single failures compromising several elements of a complex system at once. At a certain level of complexity, these common-mode events probably become the dominant accident-initiating mode, but they are particularly difficult to estimate.

In discussing the way complex systems are designed so as to minimize the possibility of accidents, it is important to recognize that accidents never derive from violations of the laws of nature, but always depend on phenomena such as unforeseen loading of a system, unforeseen deterioration of the materials, inadequate design, or faulty operations. In assessing, for example, the probability that a pipe may rupture, it is just these difficult-to-calculate fluctuations in either loading or material properties that may contribute to a failure.

It is generally recognized thai the safety of complex systems is difficult to estimate for the reason that, in addition to the reasons above, their very complexity makes it inherently difficult to calculate their behavior for a significant failure. Therefore, complex system designs generally include large safety factors and safety features specifically engineered to prevent a failure from becoming a serious accident. These safety factors, in many instances, have become standardized in engineering codes. For reactors there are special codes set up by organizations such as the American Society of Mechanical Engineers (ASME), Institute of Electrical and Electronic Engineers (IEEE), American Welding Society (AWS), and American National Standards Institute (ANSI).

Examples of specially engineered safety features are the watertight bulkheads, lifeboats, and life preservers on ocean-going ships. Large ships are designed with as many as a thousand water tight compartments, so that a single rupture below the water line will not sink the ship. In the event of a large number of underwater failures, resulting in the sinking of the ship, there are lifeboats and life preservers to save the passengers and crew. In reactors there are engineered safety features which serve the same functions: protecting the facility and protecting lives and property if part of the facility is accidentally destroyed. These include a rapidly acting SCRAM system to shut down the chain reaction, an emer- gency cooling system in case of loss of ordinary coolant, and the containment building designed to prevent the escape into the environment of radioactivity if it is accidentally released from the primary coolant system.

There is another procedure which can be used to reach an acceptable level of safety, and which is used in the aircraft industry. Although it is virtually impossible to design and build an aircraft without defects, commercial aircraft are extremely safe, and many of the safety issues are shared with reactors. The procedure in the case of aircraft, now highly standardized and bureaucratized, rests upon a careful analysis and investigation of every accident that does in fact occur, even after a detailed licensing and preproduction procedure. Corrective design modifications are then applied to all other aircraft of the same type, so that the accident is less likely to happen again. Over a period of decades this steady improvement has resulted in a generation of aircraft which could not have been designed in the beginning, but which are in fact safe enough to achieve social acceptability.

The philosophical basis of this procedure is that in a complex system there is a very wide spectrum of accidents, with the minor ones most likely, a fact borne out through experience. A rapid decrease of reactor accident probability with increasing accident severity is one of the main findings in the draft of the AEC's Reactor Safety Study (Draft WASH-1400). It is of great importance to assess the correctness of this simple result. If the decrease is rapid and if the probability of major consequences is sufficiently small, then the philosophy of learning about reactor safety through experience is sound.

\section{E. The role of licensing, regulation, and research in safety}

The $\mathrm{AEC}^{6}$ has the responsibility for the licensing and regulation of the nuclear industry. The AEC issues permits for construction of nuclear power plants and the licenses for their operation after a series of reviews to ensure that the proposed design meets standards adequate for public safety. The AEC monitors construction, fabrication, testing, and plant operation to assure compliance with the permits and licenses.

In general, these standards and criteria are well defined and clearly conservative. Many are verified through extensive industrial experience, and have been incorporated in the codes of professional societies (such as those mentioned above), while other standards and criteria have been developed through the reactor safety research program. All these criteria are published in Title 10 of the Code of Federal Regulations.

Unfortunately, it has been more difficult to establish criteria as to the adequacy of the major engineered safety features, particularly the emergency core cooling system (ECCS). These safety features are rarely used and are very difficult to test under conditions such as might occur in an accident, so there is little experience

${ }^{6}$ See Footnote 1. 
on which to base criteria. Therefore, in order for a utility to acquire construction permits and operating licenses, the reactor design must satisfy certain acceptance criteria prescribing, in many cases, rather elaborate calculational methods of evaluating the projected course of an accident, upon which the adequacy of the safety-related equipment is judged.

The course of a projected accident involves the behavior of materials and fluids in abnormal and ill-understood circumstances. Consequently, the AEC licensing organization has required very specific methods of calculation intended to be conservative at every point of uncertainty (for example, heat flow is presumed not to occur in places where it probably does). The upshot is an emphasis on calculating system behavior (purportedly conservatively) for an extremely severe hypothetical "design basis accident," rather than on making realistic assessments of the reactor behavior under abnormal conditions and requiring a conservative safety margin. The AEC has defined several design basis accidents for evaluating the designs of the engineered safety features.

Research on the course of one of these hypothetical design basis accidents, the loss-of-coolant accident (LOCA) following a break in a large cooling-water pipe, now consumes the lion's share of the budget for reactor safety research. This is largely because it is believed that emergency systems which successfully meet the requirements of this design basis accident will also provide protection against most other accidents.

We believe it particularly important that the reactor safety research program concentrate on realistic calculations and experiments and not merely on the setting of upper limits on the reactor behavior in a particular accident sequence. The latter approach would turn the research program into a demonstration program, and the reby deprive it of much of its value. Many of these questions come into focus most sharply below in connection with the hydrodynamic computer codes used to describe a large LOCA.

Perhaps a more serious problem is associated with the philosophical relationship between the design basis accident concept and incentives to reactor manufacturers for improvements in safety. The design basis accidents represent by definition the most severe accidents which the system is designed against in order to prevent serious consequences in terms of equipment damage and potential release of radioactivity. It has been observed frequently by reactor manufacturers that, if they were to provide additional safety-related equipment such as a "core catcher." or a second containment structure, the AEC might possibly redefine a design basis accident to include failure of this added equipment-thus making reactor design even more difficult than is the case with the current design basis accident. Another problem is that, if a manufacturer adds a safety feature designed to ameliorate further the consequences of a core melt (e.g., a core catcher), the AEC may not allow any credit for such an engineered feature in the safety analysis.

We believe that this situation presents a dilemma in our safety policy. Serious consideration should be given to the development of a philosophy of design standards which will provide to reactor manufacturers greater incentives to seek out methods for improving safety than does the current philosophy. While we do not attempt to spell out such a philosophy in detail, we expect that it will depend much more heavily on realistic calculations of events than does the present design-basis-accident approach and would need a strong tie to the safety research program.

\section{F. Organization of the report}

The bulk of our conclusions and recommendations are found in the main text of the report. Several of the more important appear in the Summary. The body of the report begins with Sec. III, a brief primer on light-water reactors and relevant safety considerations and procedures. Section IV contains a set of discussions on the integrity of reactor primary systems; transients (excursions from normal operating conditions); quality assurance; the contribution of human error in initiating or aggravating an incident; and sabotage.

Section V contains a discussion of some of the issues involved in the analysis of accident sequences, once some initiating event has occurred. We have examined some of the engineered safety systems in considerable detail, in particular the ECCS (emergency core cooling systems) designed to provide engineered protection to avoid the meltdown of the reactor core in the event of a loss-of-coolant accident (LOCA). Section V also contains a discussion of the consequences of a major coremelt accident, including the case where the containment is assumed to fail. This discussion provides a scale which measures the need for accident prevention, containment improvement, and mitigation of consequences and proposes possible new initiatives in these areas.

The nub of our report is Sec. VI, an evaluation of the present reactor safety research program, which is in principle designed to answer the important questions raised about reactor safety. We have studied this program in detail and are favorably impressed by the rate at which it is improving, - but are not convinced that it is as yet adequate.

Following the body of the report are Appendices, which embody supplementary detailed discussion of several important issues, as well as a list of abbreviations commonly used in the report and a full list of references. 


\section{PRIMER ON LIGHT-WATER NUCLEAR REACTORS AND RELATED SAFETY ISSUES}

This section is intended as abrief primer for those unfamiliar with nuclear power reactors and as a refresher and reference for those with some knowledge. The primer begins with a brief discussion of the nuclear reactor physics and parameters appropriate to a $1000 \mathrm{MWe}$ light-water power reactor. Following this section are brief descriptions of (i) a pressurized-water reactor (PWR) and (ii) a boilingwater reactor (BWR). These descriptions are followed by a discussion regarding the consensus that the central issue in light-water reactor safety is the prevention of a major release and dispersal of the radioactivitycontained in the reactor's fuel core. Here it is pointed out why events leading to loss of core cooling, resulting in core melt with failure of the containment, potentially have the most severe consequences. The primer then concludes with a description of the various roles of the government, nuclear reactor vendors, and utilities in the design, fabrication, and operation of nuclear power plants.

This primer draws heavily from AEC Report WASH1250 for its description of the two types of power plants and the roles of the various organizations. ${ }^{7}$

\section{A. Nuclear reactor physics}

In present day nuclear power reactors, the energy released from the fission of uranium and plutonium is used to heat water, which then runs a steam turbine which, in turn, powers an electric generator. A typical fission reaction is the following:

$$
{ }_{0}^{1} n+{ }_{92}^{235} \mathrm{U} \rightarrow{ }_{92}^{236} \mathrm{U}^{*} \rightarrow{ }_{57}^{147} \mathrm{La}+{ }_{35}^{87} \mathrm{Br}+2{ }_{0}^{1} n .
$$

There are over 40 possible fission reactions through which the intermediate excited state of ${ }_{92}^{236} \mathrm{U}^{*}$ proceeds. In Fig. 1 is shown the "fission yield," i.e., the percentage of fissions that result in fission products of a given mass number.

Most of the energy released comes from the kinetic energy of the fission products, but significant amounts come from gamma rays and the further decay of fission products, as shown in Table I. Thus the fissioning of one atom of ${ }_{92}^{235} \mathrm{U}$ leads to the release of about $212 \mathrm{MeV}$ of energy; about $200 \mathrm{MeV}$ of this is eventually turned into heat. Putting this into more practical units, complete fissioning of $1 \mathrm{~g}$ of ${ }_{92}^{235} \mathrm{U}$ releases about 1 megawatt-day of thermal energy.

In order to sustain a chain reaction, one of the neutrons emitted in the fission must go on to cause another fission, before it is captured by some nonfission process or leaks out of the reactor core. The number of neutrons emitted in a fission is given in Table $\Pi$. The average energy of the emitted neutrons is about $2 \mathrm{MeV}$, spread out over the spectrum shown in Fig. 2. The energy of these

\footnotetext{
${ }^{7}$ For more detailed descriptions of the physics and engineering of reactors there are several good texts available, e.g., S. Glasstone and A. Sesonske, Nuclear Reactor Engineering (Van Nostrand Co., 1963); D. J. Bennet, The Elements of Nuclear Power (Wiley \& Sons, 1973).
}

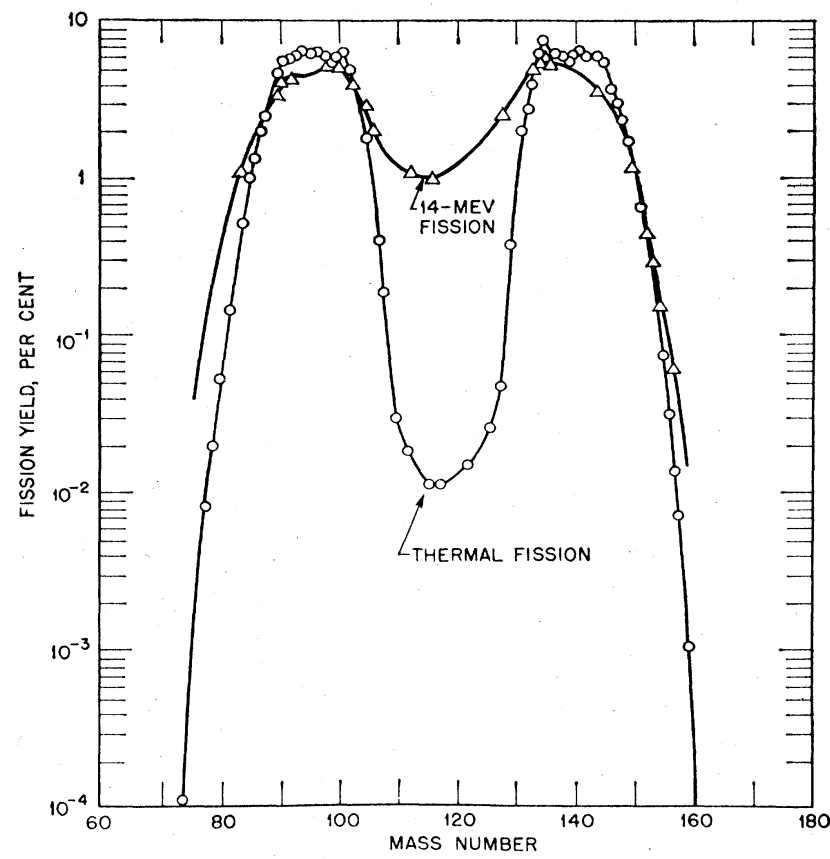

FIG. 1. Fission yield as a function of mass number (from Glasstone and Sesonske, 1963).

neutrons is too high for them to be efficient progenitors of fission. Figure 3 shows the fission cross section for ${ }^{235} \mathrm{U}$ and ${ }^{238} \mathrm{U}$ as a function of neutron energy. Note the logarithmic scale on the ordinate; a reduction in the neutron energy from $1 \mathrm{MeV}$ to $0.025 \mathrm{eV}$ (thermal energy) increases the fission cross section by nearly three orders of magnitude. Also note that the fission cross sections for ${ }^{238} \mathrm{U}$ are negligible compared to those for ${ }^{235} \mathrm{U}$ with thermal neutrons.

To take advantage of the increased fission cross section at thermal energies, many reactors (and all the reactors discussed in this report) contain a "moderating" material, chosen because of its ability to slow down neutrons through elastic collisions, while at the same time having a very low cross section for neutron absorption. The average logarithmic energy decrease per collision is called $\xi$

$$
\xi=\overline{\ln \left(E_{1} / E_{2}\right)},
$$

where $E_{1}$ is the energy of the neutron before the collision

TABLE I. End products and energies from fission of ${ }^{235} \mathrm{U}$ (from Bennet, 1973).

\begin{tabular}{lc}
\hline \multicolumn{1}{c}{ End-product } & $\begin{array}{c}\text { Emitted energy } \\
(\mathrm{MeV})\end{array}$ \\
\hline Fission products & 168 \\
Fission neutrons & 5 \\
Prompt $\gamma$ radiation & 7 \\
Fission product decay & \\
$\beta$ radiation & 8 \\
$\gamma$ radiation & 7 \\
neutrinos & 12 \\
Capture $\gamma$ radiation & 5 \\
\hline Total & 212 \\
\hline \hline
\end{tabular}


TABLE II. The number of neutrons emitted per fission (from Bennet, 1973).

\begin{tabular}{lll}
\hline \hline Isotope & $\begin{array}{c}\text { Incident } \\
\text { neutron energy }\end{array}$ & $\begin{array}{c}\text { Number of neutrons } \\
\text { emitted per fission }\end{array}$ \\
\hline${ }^{235} \mathrm{U}$ & $0.025 \mathrm{eV}$ & 2.44 \\
${ }^{239} \mathrm{Pu}$ & $1 \mathrm{MeV}$ & 2.50 \\
& $0.025 \mathrm{eV}$ & 2.87 \\
${ }^{233} \mathrm{U}$ & $1 \mathrm{MeV}$ & 3.02 \\
${ }^{232} \mathrm{Th}$ & $0.025 \mathrm{eV}$ & 2.48 \\
${ }^{238} \mathrm{U}$ & $1 \mathrm{MeV}$ & 2.55 \\
\hline \hline
\end{tabular}

with the moderating nucleus, and $E_{2}$ is the energy after the collision. Here $\xi$ can be calculated from the kinematics of the collision. The most useful figure of merit for moderators is the "moderating ratio," which is $\xi \cdot \Sigma_{s} /$ $\Sigma_{a}$, where $\Sigma_{s}$ is the macroscopic scattering cross section, and $\Sigma_{a}$ is the macroscopic absorption cross section. The moderating ratio for several moderators is shown in Table III. The first reactor built by Fermi at Chicago used graphite; Canadian power reactors use heavy water; power reactors in the U. S. use ordinary "light" water (hence the terminology "light-water reactor," LWR). The average number of collisions necessary to reduce the energy of a neutron from energy $E_{a}$ to energy $E_{b}$ is $N=\ln \left(E_{a} / E_{b}\right) / \xi$, if binding effects are neglected. Thus to reduce $2 \mathrm{MeV}$ neutrons to $0.025 \mathrm{eV}$ when using light water $(\xi \simeq 1)$ requires about 18 collisions. This will take a "slowing down time" of about $10^{-5} \mathrm{sec}$. After slowing down, the neutron will continue to diffuse (scatter) until it is absorbed by a nucleus or until it leaks out of the reactor. The time spent slowing down and diffusing in various moderators is shown in Table IV.

It is worthwhile to point out here that the preceding numbers imply that it is physically impossible for a moderated reactor to "explode like an atomic bomb." The chain reaction in normal light-water reactor operation relies on the $\approx 1 \%$ fraction of the fission neutrons which are emitted by some of the fission fragments a few seconds after fission. Thus there is a built-in delay time of several seconds between the absorption of a neutron to cause fission, and the emission of $100 \%$ of the additional

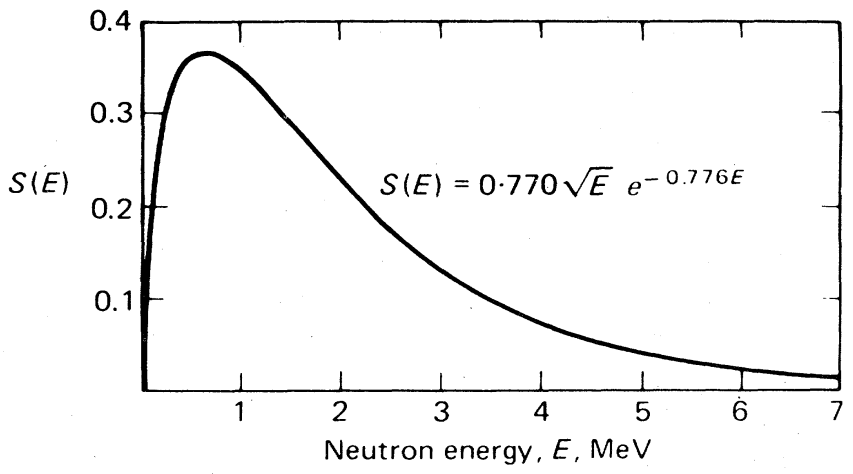

FIG. 2. The fission neutron energy spectrum (from Bennet, 1973).

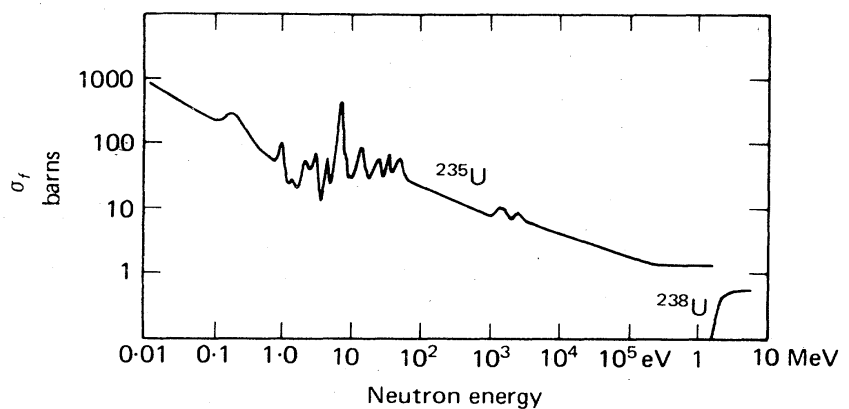

FIG, 3. Fission cross sections of ${ }^{235} \mathrm{U}$ and ${ }^{238} \mathrm{U}$ (from Bennet, 1973).

neutrons necessary to sustain fission. If the moderator is suddenly removed (due, for example, to a major pipe rupture, a "loss-of-coolant accident") then the chain reaction will stop. In addition, if an "explosion" began, the shock wave would disperse the nuclear material faster than the velocity of thermal neutrons (about $2000 \mathrm{~m} / \mathrm{s}$ ); this lack of inertial constraints means that the release of energy would fizzle. Furthermore, the decrease in density of water with temperature helps stabilize reactor operations. A surge in reactivity by increasing the temperature decreases the density and hence the moderating power of the water, which then leads to a reduction in reactivity. The conceivable dangers from a large nuclear reactor arise not from a release of energy, but from the possible release of the large amount of radioactivity in the fission fragments present in such a reactor.

The chain reaction can be halted at any time by inserting neutron-absorbing materials into the core of the reactor. The capture cross section for thermal neutrons for several materials is given in Table $V$. The reactivity of the reactor can be adjusted by the controlled insertion of materials such as cadmium. One of the emergency shutdown systems, SCRAM, involves the injection of borated water into the core of the reactor.

Natural uranium consists of about $0.715 \%$ of ${ }^{235} \mathrm{U}$ and $99.285 \%$ of ${ }^{238} \mathrm{U}$. As discussed above, the ${ }^{238} \mathrm{U}$ is useless for sustaining a chain reaction (although it is a useful target for neutrons for the production of plutonium). ${ }^{238} \mathrm{U}$ has several resonances in its neutron absorption cross section that rapidly reduce the number of neutrons passing through those energy regions while being thermalized. Unless a moderator with unusually low absorption

TABLE III. Slowing down properties of moderators (from Glasstone and Sesonske, 1963).

\begin{tabular}{lcr}
\hline \hline Moderator & $\begin{array}{c}\text { Slowing } \\
\text { down power } \\
\xi \cdot \Sigma_{s}\end{array}$ & $\begin{array}{c}\text { Moderating } \\
\text { ratio }\end{array}$ \\
\hline Water & 1.28 & 58 \\
Heavy water & 0.18 & 21000 \\
Helium a & $10^{-5}$ & 45 \\
Beryllium & 0.16 & 130 \\
Graphite & 0.065 & 200 \\
\hline \hline
\end{tabular}

${ }^{a}$ At atmospheric pressure and temperature. 
TABLE IV. Slowing down and diffusion times for thermal neutrons in an infinite medium (from Glasstone and Sesonske, 1963).

\begin{tabular}{lccc}
\hline \hline Moderator & $\Sigma_{s}\left(\mathrm{~cm}^{-1}\right)$ & $\begin{array}{c}\text { Slowing down time } \\
(\mathrm{sec})\end{array}$ & $\begin{array}{c}\text { Diffusion time } \\
(\mathrm{sec})\end{array}$ \\
\hline Water & 1.40 & $7.1 \times 10^{-6}$ & $2.4 \times 10^{-4}$ \\
Heavy water & 0.35 & $5.0 \times 10^{-5}$ & $6.0 \times 10^{-2}$ \\
Beryllium & 0.75 & $5.7 \times 10^{-5}$ & $4.2 \times 10^{-3}$ \\
Graphite & 0.41 & $1.4 \times 10^{-4}$ & $1.6 \times 10^{-2}$ \\
\hline \hline
\end{tabular}

cross section is used (such as heavy water in the Canadian power reactors), it is necessary to enrich the ${ }^{235} \mathrm{U}$ content of the fuel. In light-water power reactors in the U. S. uranium is enriched to contain $2 \%$ to $4 \%{ }^{235} \mathrm{U}$. In addition, the uranium fuel is spatially separated from the water moderator, allowing the fission neutrons to escape into the moderator and slow down, with a smaller chance of absorption, before diffusing back to the fuel.

\section{B. Description of a commercial light water reactor}

There are two basic types of light-water reactors in common use in the United States today: pressurizedwater reactors (PWR) and boiling-water reactors (BWR). Figures 4 and 5 show, in schematic form, the elemental components of BWR and PWR power generating systems. As indicated in the figures, the principal difference between the two reactor systems is related to the relative isolation of the nominally weakly radioactively contaminated working fluid of the reactor from the turbine-generator steam supply.

In the BWR, as shown in Fig. 4, steam generated by direct boiling in the nuclear core passes directly to the turbine generators. It carries along with it whatever radioactivity may be normally produced in the core either from neutron interactions with trace contaminants in the water or from leakage of fission products from pinhole openings in the fuel rods. The BWR working fluid is continually operated on by radioactive cleanup processes to remove these trace contaminants.

In the PWRs, as shown in Fig. 5, steam for the turbines is produced in a secondary heat exchange loop isolated from the nominally radioactively contaminated primary system of the reactor core. High-pressure water, maintained at temperatures at which bulk boiling will not occur, circulates through the primary loop of the reactor core and steam generator. At relatively lower temperatures and pressures, steam is generated for the turbines and as an isolated fluid circulates in the secondary loop of the steam generator.

In a commercial light-water reactor (LWR), the enriched uranium fuel is in the form of uranium dioxide $\left(\mathrm{UO}_{2}\right)$ pellets encased in tubular metal cladding. Clusters of this tubing form the core of the LWR. Water circulates between these fuel elements to provide both moderation and cooling. The fuel rod and water combination is contained in a heavy reactor vessel, with large inlet and outlet nozzles for the coolant water. Also contained
TABLE V. Capture cross sections in various materials.

\begin{tabular}{lc}
\hline \multicolumn{1}{c}{ Material } & Capture cross section $\left(10^{-24} \mathrm{~cm}^{2}\right)$ \\
\hline Natural uranium & 7.6 \\
Water & 0.66 \\
Heavy water & 0.001 \\
Boron & 759 \\
Cadmium & 2450 \\
\hline \hline
\end{tabular}

in the reactor vessel are control rods, which can be inserted into the core of the reactor to absorb neutrons and thus control the chain reaction. The water coolant is driven from the reactor vessel to the heat exchanger (or, for a BWR, to the turbines) by large pumps. The pumps are necessary to remove from the core the intense heat from the continuing chain reaction. However, if the chain reaction is stopped, natural convection will remove sufficient heat from the core if the power to the pumps should fail.

A 1000 megawatt commercial power reactor will typically contain 87 metric tons of uranium fuel. Operating parameters for such a reactor are shown in Table VI. In the sections that follow we will describe commercial PWRs and BWRs in more detail, with emphasis on those elements relating to safety.

\section{Pressurized-water reactor (PWR) description}

A cross-sectional view of a typical PWR is shown in Fig. 6. The figure provides perspective on the relative sizes of nuclear steam supply (NSS) components and their layout in a typical operational configuration. Figure 7 presents a schematic diagram of the NSS and its associated emergency core cooling systems (ECCS) equipment, designed to minimize damage to the reactor from a loss-of-coolant-accident (LOCA). The internal fluid pressure of a typical large PWR is maintained at about 2200 pounds per square inch (psi), at which pressure water can be heated to nearly $650^{\circ} \mathrm{F}$ without boiling. To allow high heat transfer rates without bulk boiling, this high-pressure water is heated to an average temperature of only about $600^{\circ} \mathrm{F}$. It is then piped from the reactor vessel to two or more devices called steam generators. Here the high-pressure reactor coolant water circulates through tubes whose outer surfaces are in contact with a second stream of water returning from the turbine condenser. The latter water stream is at considerably lower pressure and temperature than the former, and heat transferred from the hot water inside the tubes causes the water of the secondary stream to boil and produce steam for the turbine at typical pressures of 1000 psi or less and temperatures of about $500^{\circ} \mathrm{F}$.

All PWRs employ this dual system for transferring energy from the reactor fuel to the turbine and are called "indirect cycle" systems. The high-pressure circuit, comprising the reactor vessel, piping, the necessary pumps, and the inner tube side of the steam generators, is termed the "primary system"; the lower pressure circuit which delivers steam to the turbines is called the "secondary system." 
FIG. 4. Schematic idealization of boiling-water reactor. power system components.

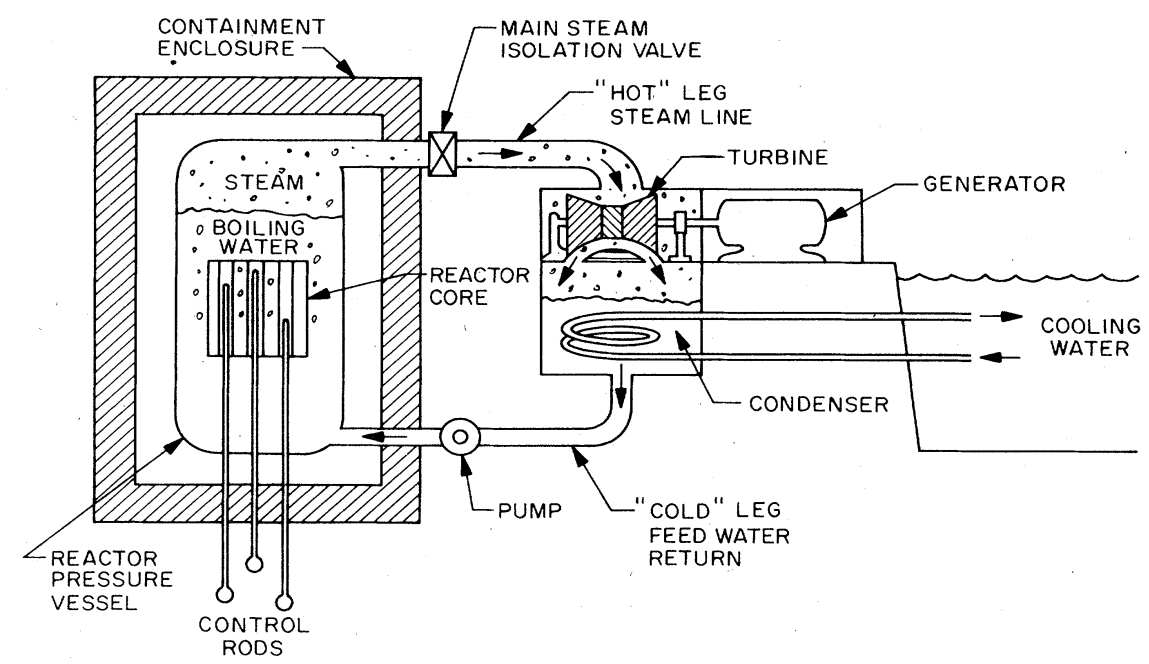

\section{a. Reactor vessel}

PWR reactor vessels are cylindrical in shape, some being over 40 feet high, with an inside diameter of over $14 \mathrm{ft}$ and wall thickness in excess of 8 inches. The vessels have removable top heads (for refueling) provided with fittings to accommodate the mechanisms for driv ing neutron-absorbing rods into and out of the core to control the nuclear chain reaction.

\section{b. Fuel}

Large PWRs contain nearly 40000 fuel rods, totaling about 100 tons of slightly enriched uranium dioxide. For the larger PWRs, 176 to 220 fuel rods are assembled into a bundle of square cross section which is typically about 8.5 inches on a side. PWR fuel assemblies are relatively open arrays allowing radial mixing of the primary fluid as it passes through the reactor.

\section{c. Steam generators}

There are various designs for steam generators. One common design is called a vertical U-tube heat exchanger (shown schematically in Fig. 7) in which the heated water from the reactor enters near the bottom, passes through literally thousands of U-shaped tubes, and finally leaves near the same elevation at which it entered. The upper section of these steam generators contains equipment to separate small quantities of liquid water droplets from the raw steam; such droplets are invariably carried along with the steam from the boiling fluid. The lower section of the steam generator in which boiling occurs is called the evaporator section, and the upper section in which the steam is separated from the suspended water droplets is called the steam drum section.

To give an idea of steam generator size and complexity, the following data apply to the steam generators used in a typical large PWR plant. Each of the steam generators

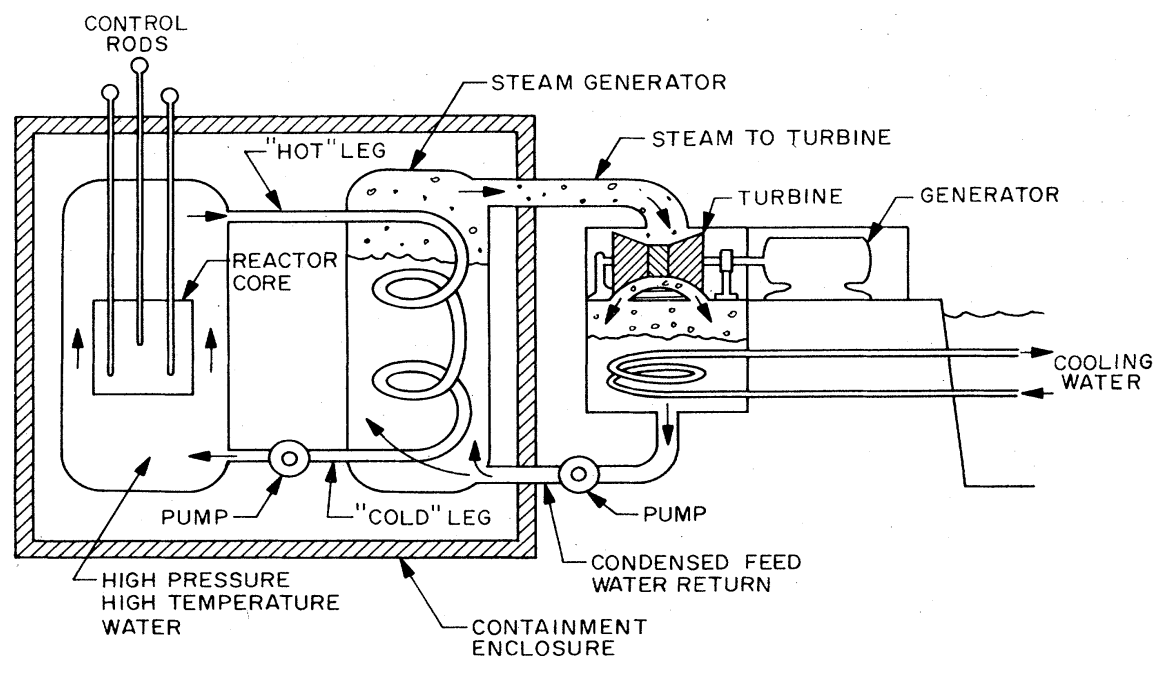

FIG. 5. Schematic idealization of pressurized-water reactor power system components. 
TABLE VI. Typical operational parameters for 1100 MWe light-water reactors.

\begin{tabular}{|c|c|c|}
\hline Parameters & PWR & BWR \\
\hline Thermal power rating (MWt) & 3400 & 3300 \\
\hline Electrical power output (MWe) & 1100 & 1100 \\
\hline \multicolumn{3}{|l|}{ Reactor } \\
\hline System pressure (psi) & 2200 & 1000 \\
\hline Inlet temperature $\left({ }^{\circ} \mathrm{F}\right)$ & 550 & 420 \\
\hline Outlet temperature $\left({ }^{\circ} \mathrm{F}\right)$ & 600 & 550 \\
\hline Coolant flow (millions of $1 \mathrm{~b} / \mathrm{hr}$ ) & 140 & 110 \\
\hline \multicolumn{3}{|l|}{ Turbine operating conditions } \\
\hline Inlet pressure (psi) & 800 & 1000 \\
\hline Inlet temps. $\left({ }^{\circ} \mathrm{F}\right)$ & 500 & 550 \\
\hline \multicolumn{3}{|l|}{ Heat transfer at rated power } \\
\hline Active surface area $\left(\mathrm{ft}^{2}\right)$ & 53000 & 66000 \\
\hline Avg. heat flux (Btu/hr-ft ${ }^{2}$ ) & 210000 & 160000 \\
\hline Max. heat flux $\left(\mathrm{Btu} / \mathrm{hr}-\mathrm{ft}^{2}\right)$ & 560000 & 420000 \\
\hline Avg. linear heat rate $(\mathrm{kW} / \mathrm{ft})$ & 7 & 7 \\
\hline Max. linear heat rate $(\mathrm{kW} / \mathrm{ft})$ & 19 & 18 \\
\hline \multicolumn{3}{|l|}{ Initial core loading } \\
\hline \multicolumn{3}{|l|}{ Initial charge (metric tons } \\
\hline of uranium) & 87 & 149 \\
\hline Assemblies/core & 193 & 764 \\
\hline Rods/assembly & 204 & 49 \\
\hline Annual discharge & $\frac{1}{3}$ Core & $\frac{1}{4}$ Core \\
\hline Fuel (metric tons of uranium) & 29 & ${ }^{4} 37$ \\
\hline Assemblies (number) & 64 & 190 \\
\hline Fuel rods (number) & 13000 & 9300 \\
\hline \multicolumn{3}{|l|}{ Fuel rods } \\
\hline Number & 39000 & 37000 \\
\hline Outside diam (in) & 0.43 & 0.56 \\
\hline Cladding thickness (in) & 0.025 & 0.03 \\
\hline Fuel pellet diam (in) & 0.37 & 0.49 \\
\hline Diametral gap (in) & 0.01 & 0.01 \\
\hline Active length (in) & 144 & 144 \\
\hline
\end{tabular}

has an overall height of 63 feet with an upper shell (where the steam drum and drying equipment are located) 174 inches (14.5 feet) in diameter, and lower shell (where the evaporator section is located) with a diameter of 135 inches (11.25 feet). There are $3260 \mathrm{U}$-tubes through which the primary coolant is pumped in each steam generator. Since the steam generators are so large and operate under high pressure, they must be fabricated from thick steel plate in order to have adequate structural strength. This makes the steam generators among the most massive and sizeable objects in the plant.

\section{d. Primary coolant pumps}

The primary coolant pumps circulate the water through the primary coolant system, that is, between the reactor and the steam generator. High reliability and performance are demanded of the primary system reactor coolant pumps since they (a) provide vital coolant to the reactor and (b) are critical to operation of the steam generators. Because the pumps must operate at high temperatures and pressures, and because they circulate water which is somewhat radioactive, the stringent design and manufacturing criteria used for the other primary components must apply to the pumps. In a typical 1000 MWe PWR each of the four primary pumps is about $30 \mathrm{ft}$ high, is driven by a 6600 horsepower electric motor, and has a pumping capacity of about 88,500 gallons per minute. The pump casings are designed for operation at $650^{\circ} \mathrm{F}$ at a pressure of 2500 psi. Each PWR employs more than one recirculation loop and pump to meet its cooling requirements (i.e., redundancy in number though not in capacity) and each pump has a capacity greater than that required to accommodate removal of the decay heat load immediately following a reactor shutdown.

\section{e. Pressurizers}

PWR steam supply systems are equipped with pressurizers to maintain constant primary coolant pressure during operation and to limit pressure changes caused by coolant thermal expansion and contraction as plant loads change.

A typical pressurizer for a large plant contains immersion-type electric heaters of about $2000 \mathrm{~kW}$ capacity, multiple safety and relief valves, and a spray nozzle, plus appropriate valves and instruments. The lower portion of the pressurizer contains liquid water and the upper portion contains steam. The pressurizer is connected by a surge line (pressure stabilizer) joining the pressurizer to the hot side (leg) of one of the reactor coolant loops. When the plant electric load is decreased, the temperature of the primary coolant rises and a positive pressure surge in the primary system results in automatic operation of the spray system in the top of the pressurizer; this condenses some of the steam and keeps the pressure below that which would operate the pressure relief valves. During a negative pressure surge, caused by an increased plant electrical load, the electric heaters are turned on and sufficient steam is generated within the pressurizer to keep the primary system pressure above the minimum allowable limit. Like the reactor vessel, the steam generators, the pumps, and all other parts of the primary system, the pressurizer is also located within the containment. The pressurizer is shown schematically in Fig. 7.

\section{f. PWR emergency core cooling system (ECCS)}

Each nuclear plant has many different features that are provided to compensate for the possibility of operator errors, equipment defects, abnormal occurrences, and a variety of accidents. In addition, each plant has a number of backup safety devices and systems to protect against damage to the plant or harm to operators and to the general public. In a nuclear power reactor, the fission reaction that produces most of the heat can be turned off by inserting the control rods into the reactor core. However, the radioactive decay of the fission products produced within the fuel during normal operation continues to produce substantial amounts of heat, and this source cannot be turned off. The fission product decay heat amounts to approximately $7 \%$ of the rated thermal output of the plant at shutdown, but decays rather rapidly for a short time thereafter. The decay heat output has decreased to $5 \%$ by 10 seconds after shutdown, and drops to $2 \%$ after 10 minutes, $1 \%$ after 2.25 hours, and within 24 hours has fallen to only about $0.5 \%$ of the rated thermal output of the plant (assuming extended normal plant operation at or near its rated capacity prior to shutdown). A system must be provided 
FIG. 6. Typical PWR containment, from Unit 2 Diablo Canyon, Pacific Gas and Electric Company.

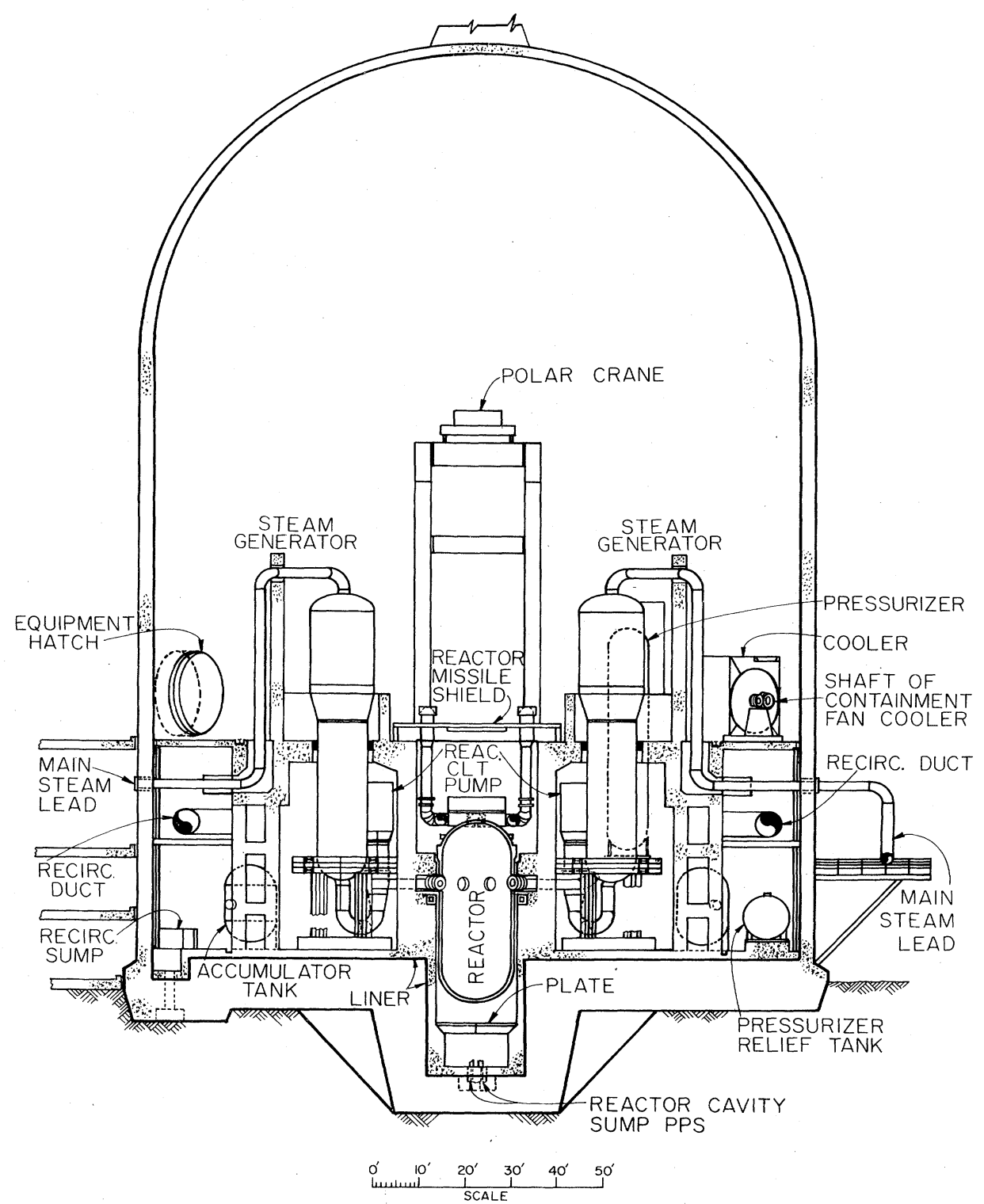

to cool the core and to prevent the fuel rods from melting if normal cooling is interrupted. This emergency core cooling system is one of the more important of the protective systems and is described briefly here.

The major function of the emergency core cooling system of a PWR is to supply sufficient water to cool the core in the event of a break that might leave the core uncovered. The ECCS must be capable of supplying fluid for any break from a small crack to a rupture of the largest coolant pipe in the system. PWR emergency core cooling systems consist of several independent subsystems, each characterized by redundancy of equipment and flow path. This redundancy is intended to assure reliability of operation and continued core cooling even in the event of failure of any single component.

The primary element of the ECCS is an accumulator injection system. It consists of two or more large tanks that are connected through check valves and piping to the main coolant pipes between the primary coolant pumps and the reactor vessel or to the reactor vessel itself. These tanks contain cool borated water stored under nitrogen gas at a pressure of 200 to 650 psi. If a large break were to occur in the reactor primary system, the hot water would be expelled through the break and the pressure in the primary system would decrease rapidly.

If the pressure were to become less than the gas pressure in the accumulators, the check valves would open and discharge water from the accumulators into the reactor vessel to cool the core. The accumulator injector system is a passive system. That is, it functions automatically without activation of pumps, motor driven valves, or other equipment.

Two active systems are also incorporated to provide emergency core cooling. One is a low-pressure system for use in the event of large breaks, which could produce rapid depressurization and ejection of the bulk of the 


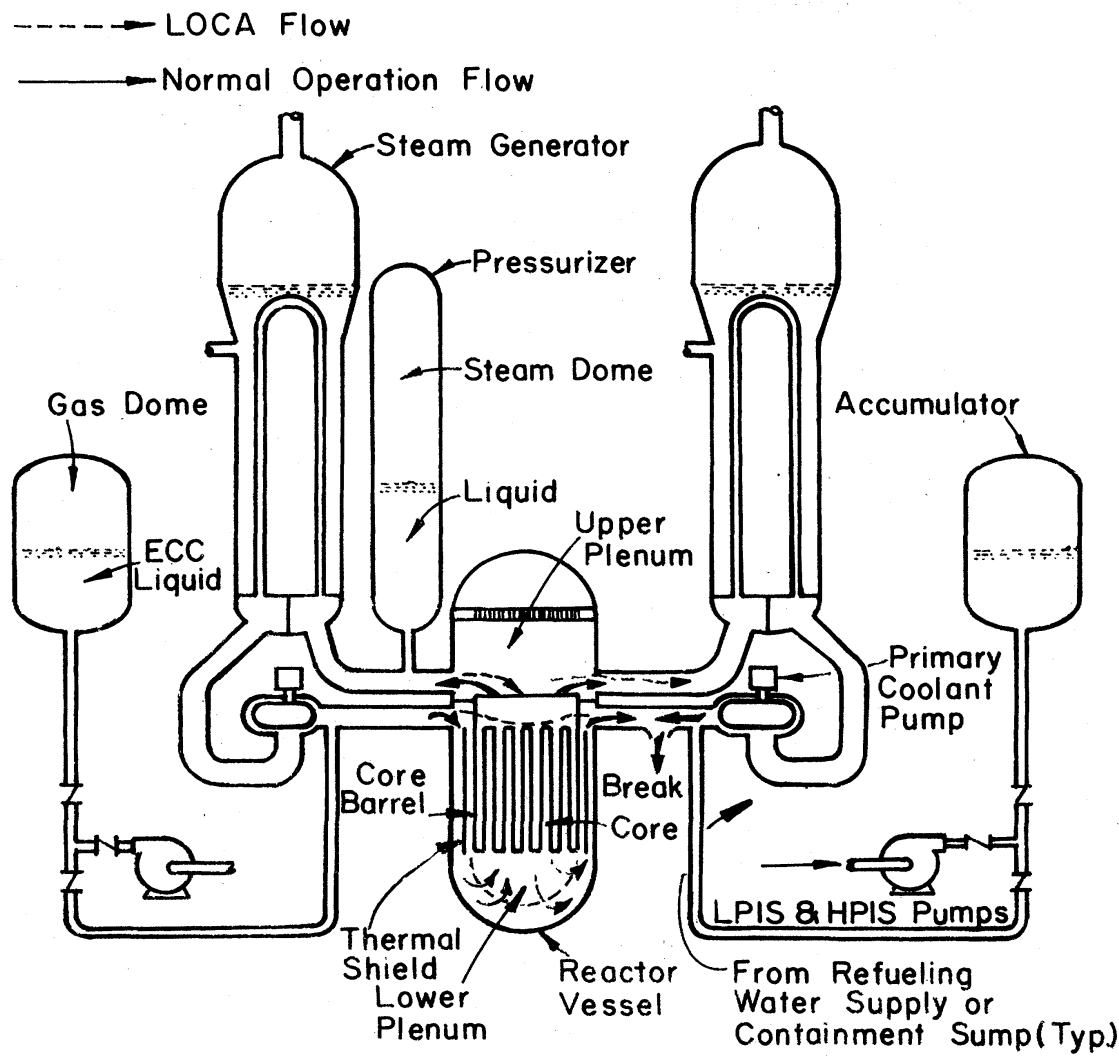

FIG. 7. Schematic diagram of PWR steam supply system showing emergency core cooling system (ECCS) elements.

primary coolant. This low-pressure system would continue to inject emergency core coolant (ECC) from the refueling water storage tank and ultimately from pumps within the drywell of the containment vessel into the primary system for long times after the above-mentioned accumulators are empty. The other is a high-pressure system to supply supplemental coolant if the break is small and the primary coolant pressure remains high. The active systems have pumps and valves that must operate if the systems are to function properly. Power to operate the active ECC systems is obtainable from several sources. An active system is energized automatically by coolant pressure sensing and level sensing switches whose operation will connect power (normal or emergency) to the appropriate pumps and valves and thereby initiate emergency core cooling.

Although the arrangements and designs of PWR emergency core cooling systems vary from plant to plant, depending on the vendor of the steam supply system, all modern PWR plants employ both accumulator injection systems and pump injection systems, with redundancy of equipment which is designed to assure reliability of operation.

\section{g. Containment}

The primary system of a PWR contains large amounts of radioactive fission products. A leak in a pipe could the refore result in the release of radioactive material to the surroundings, so that the entire primary system must be installed in a special building that is capable of holding the fission products. Such a building is called the containment building.

In normal plant operation, the containment building retains the low-level routine radioactive emissions for processing in hold-up tanks for decay, and then releases them through filters. Since PWR containment buildings are typically 200 feet high by 125 feet in diameter and have hundreds of penetrations, they are never perfectly gastight but are required not to exceed a specified (very low) leak rate. Refinements in containment technology are still being made and containment systems vary widely from plant to plant. For example, in some PWR plants, the containment space is kept at slightly below atmospheric pressure so that normal leakage through the containment walls is inward; other plants have double barriers against escape of material from the containment. Most present day PWR containments are constructed of reinforced concrete with a steel liner, and are what the public usually sees when viewing a nuclear reactor from off-site.

In the event of a loss-of-coolant accident (LOCA), the expelled primary coolant would flash to steam and pressurize the containment volume. Containment buildings are sized and designed to withstand the maximum temperature and pressure expected from the steam generated, although some PWR containments may not be able to 
withstand the pressure generated in an accident involving loss of both primary and secondary cooling water.

Two kinds of additional measures are taken in PWR plants to minimize the potential for escape to the environment of any accidental release of radioactivity. In some plants cold-water sprays are provided to condense the steam if there should be a major escape of primary system coolant into the containment; in others stored ice is used. By condensing the steam, and thus lowering the containment pressure, the driving force for outward leakage is reduced. This is important since in a major accident the significant radioactivity held in the primary coolant would be released into the containment, and if the core should melt, large quantities of radioactive materials would become airborne in the containment.

Another safety measure provides blowers to recirculate the containment atmosphere through filters and absorption beds, to remove airborne radioactive materials. When sprays are used in the containment, chemicals are usually added to the spray solution to increase the retention of airborne radioactive materials that dissolve in and become entrained by the spray. Systems for the control of hydrogen from both metal-water reactions and radiolytic decomposition of the water are also provided to assure that flammable gas concentrations are not reached within the containment.

\section{Boiling-water reactor (BWR) description}

A cutaway drawing of a BWR is shown in Fig. 8. A more detailed, but schematic, diagram of the BWR nuclear steam supply system is presented in Fig. 9. The nuclear steam supply system consists primarily of the reactor vessel and equipment inside the vessel. The nuclear fuel assemblies are arranged inside a core shroud in the reactor vessel. Water boils in the core and a mixture of steam and water flows out the top of the core and through steam separators at the top of the core shroud. Steam from the separators passes through dryers to remove entrained water and then leaves the reactor vessel through pipes to the turbine generators. Water from the steam separators mixes with the water returned from the turbine condenser, flows downward through the annulus between the core shroud and the reactor vessel, and returns to the bottom of the core. Because the energy supplied to the reactor coolant water from the hot fuel is transported directly (without an intermediate heat exchange process) as steam to the turbine, the BWR system is termed a "direct cycle" system. The pressure in a typical BWR is maintained at about 1000 psi. At this pressure water boils and forms steam at about $545^{\circ} \mathrm{F}$.

\section{a. Nuclear steam supply system}

Except for size, the nuclear steam supply systems (NSSS) of all BWR plants in the United States are essentially similar. Typically, a BWR of 1000 MWe size has a reactor vessel about 21 feet in diameter, over 72 feet in height (about three times the volume of a typical PWR pressure vessel), with a wall thickness of 6 to 7 inches. Details of the operational characteristics of a typical BWR are given in Table VI. Steam flows from the reactor vessel to the turbine generator in multiple main steam lines. The head of the vessel and the steam separators and dryers are removable for refueling the core. Neutron absorption control and safety elements in the reactor core are connected to rods that pass through fittings in the bottom head of the vessel and are operated by hydraulic drives mounted below the vessel. Because the reactor heat output is sensitive to the rate of flow of coolant through the core, partial control of the power is effected by varying the driving flow to the jet pumps and thereby the rate that the jet pumps recirculate water through the core.

\section{b. Fuel}

Fuel rods for BWRs are similar to those for PWRs. They are about one-half inch in diameter with about 12 feet of active fuel length sealed within zircaloy tubular cladding. Forty-nine fuel rods (64 rods in fuel assemblies for the most recent BWR/6 system) are installed in a metal channel of square cross section to form a fuel assembly. The channel is open at the top and bottom to permit coolant to flow upward through the as sembly; however, the closed sides prevent lateral flow of coolant between adjacent assemblies in the reactor core. The core of a large BWR of current design may contain as many as 764 fuel assemblies (at 49 rods per assembly this is more than 37,000 fuel rods per reactor) with a total weight of uranium dioxide of more than 149 tons.

\section{c. Primary coolant pumps}

The rate at which heat can be extracted from a reactor core of a given size depends on the rate of recirculation of water through the core. In large BWRs, jet pumps are provided in the annulus outside the core shroud to greatly increase the circulation rate over the natural circulation induced by the boiling in the core. Water is withdrawn from the annulus by motor driven recirculation pumps outside the reactor vessel and is returned to provide the driving flow for the jet pumps. The jet pumps deliver water from the annulus to the bottom of the core and provide the pressure to drive the flow through the fuel assemblies.

\section{d. BWR emergency core cooling system}

BWRs, like PWRs, have multiple provisions for cooling the core fuel in the event of an unplanned depressurization or loss of coolant from the reactor. The provisions may differ from plant to plant, but all plants have several independent systems to achieve flooding and/or spraying of the reactor core with coolant upon receiving a signal of either high drywell pressure or low reactor vessel water level.

Typical BWR emergency core cooling systems consist (in part) of a high-pressure core spray system (early BWRs) or a high-pressure coolant-injection system (later BWRs) which are provided to assure adequate cooling of the core in the event of a small leak that results in slow depressurization of the reactor system. If such a leak should occur, the reactor would shut down quickly on receipt of a signal of low water level in the reactor vessel. Failure of the feedwater pumps or other 


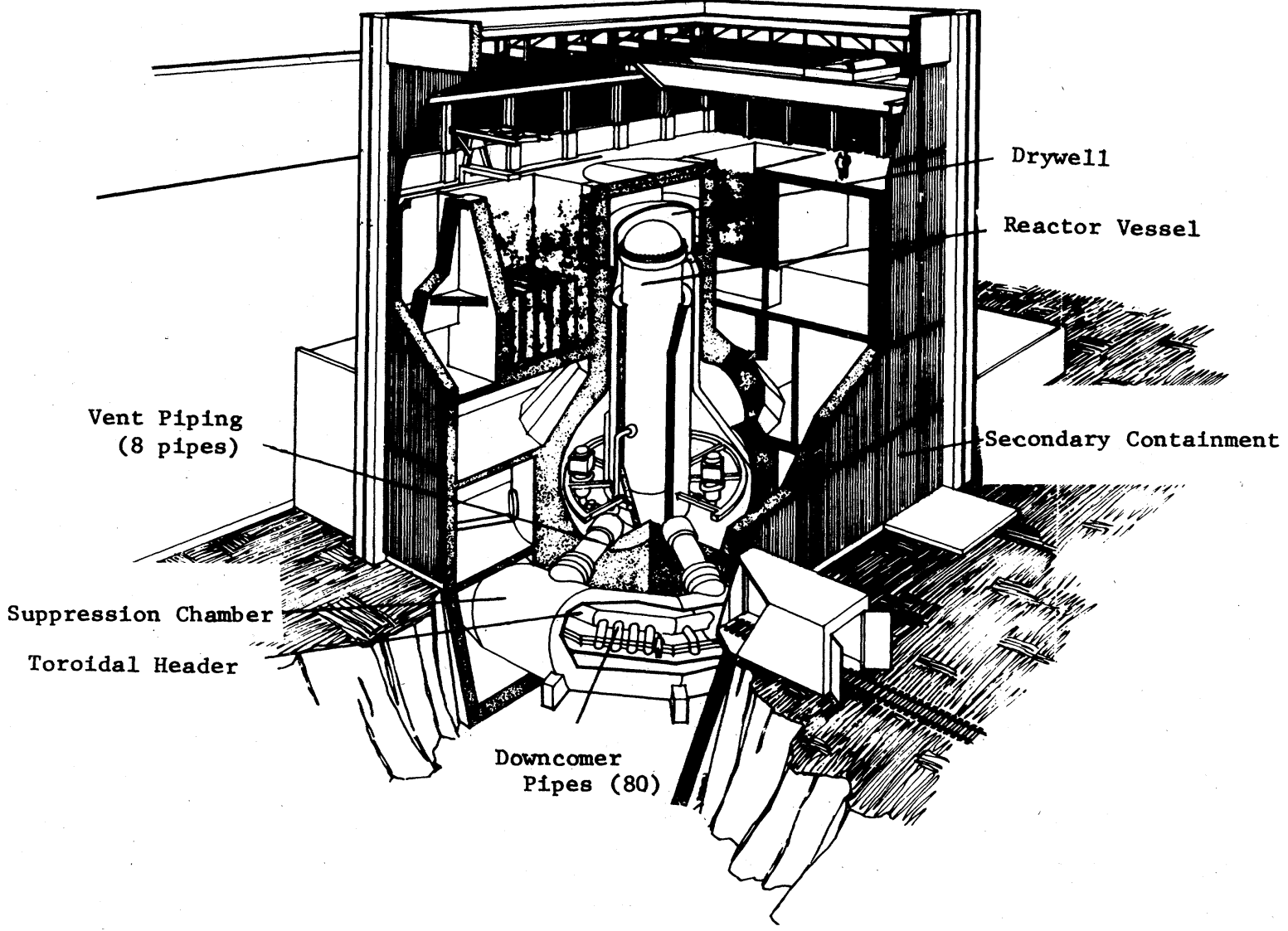

FIG. 8. BWR secondary containment building showing primary containment system enclosed (from AEC, WASH-1250, 1973).

minor systems which supply water to the reactor during normal operation to maintain the water level above a preselected height above the core, or the detection of high pressure in the drywell containment outside the reactor pressure vessel, would initiate automatic action to bring the high-pressure injection system into operation. The high-pressure coolant pumps are driven by steam turbines. Full pump speed and flow can be developed in 25 seconds.

If, for any reason, the feedwater pumps and the highpressure emergency cooling systems should be incapable of maintaining the desired reactor water level, an automatic depressurization system would operate to discharge steam through pressure relief valves into the pressure suppression pool system, thereby lowering the pressure in the reactor so that operation of the lowpressure emergency cooling systems could be initiated. (The pressure relief valves would automatically open upon coincident signals of low water level in the reactor vessel and high pressure in the primary containment.)

A low-pressure core spray system uses two independent loops to provide emergency cooling for use after the reactor has been depressurized. Each loop has two electrically driven (with power available from both normal and emergency sources) centrifugal pumps, and each connects through separate piping to a separate spray header above the core. These systems spray water onto the fuel assemblies at flow rates sufficient to cool the fission product decay heat of the core unassisted. Another independent system, the low-pressure coolant-injection system, is provided to supplement the low-pressure core spray system providing the principal fluid source to reflood the core. This latter coolant injection system utilizes independent pumps and has adequate capacity to protect the core following even a large break.

\section{e. Containment}

Containment systems of current design BWRs generally provide both "primary" and "secondary" containment. The former is a steel pressure vessel surrounded by reinforced concrete and designed to withstand peak transient pressures which might occur in the most severe of the postulated, though unlikely, loss-of-coolant accidents. This primary containment employs a "drywell," enclosing the entire reactor vessel and its recirculation pumps and piping, which is connected through large ducts to a lower-level pressure-suppression chamber which stores a large pool of water, as shown in Fig. 8. Under accident conditions, valves in the main steam lines from the reactor to the turbine generators would automatically close, and any steam escaping from the 
FIG. 9. Schematic diagram of BWR steam supply system showing emergency core cooling system (ECCS) elements.

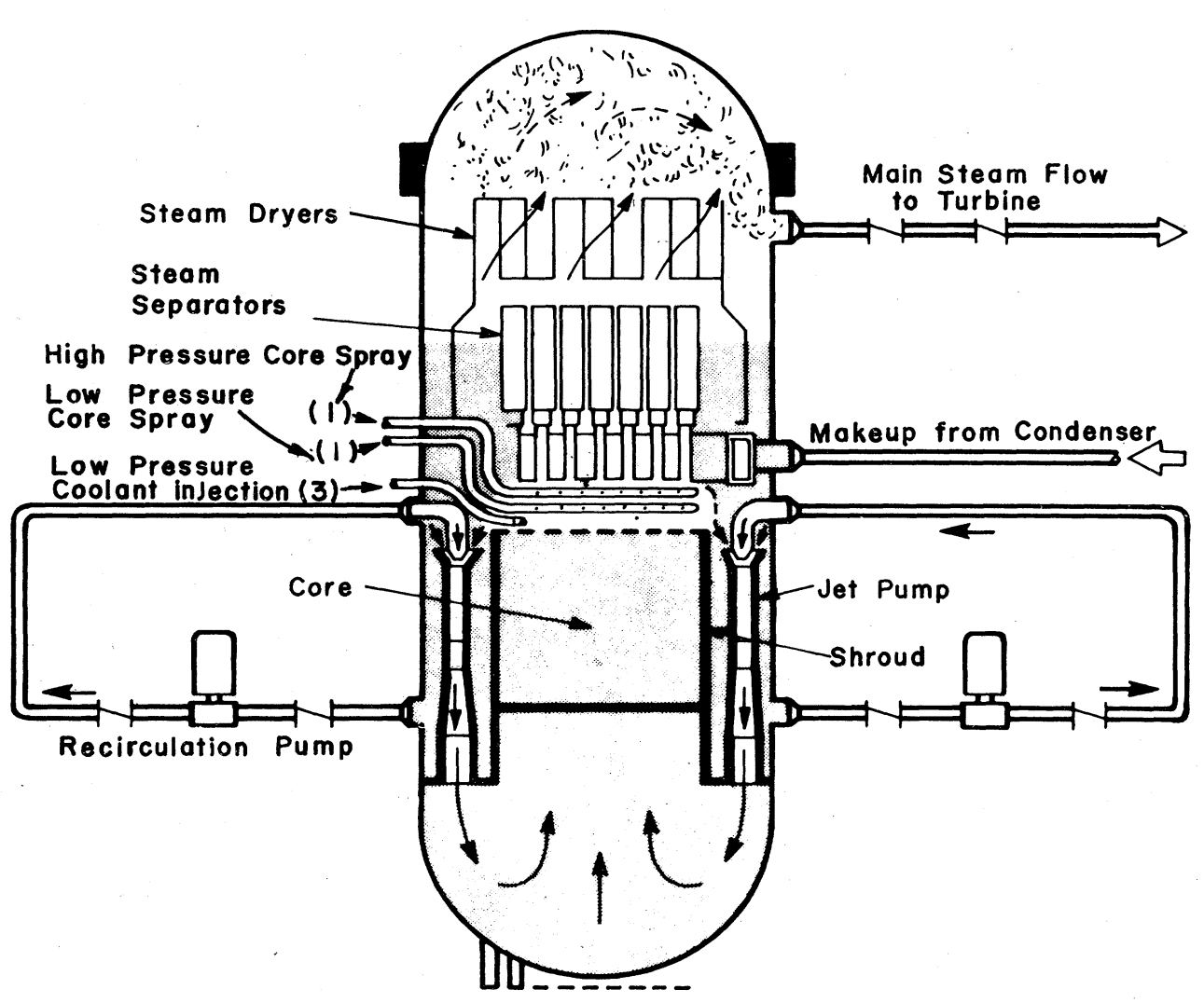

reactor system would be released entirely within the drywell. The resulting increase in drywell pressure would force the air-steam mixture in the drywell down into and through the water in the pressure-suppression chamber, where most of the steam would be condensed. Steam released through the pressure relief valves of the automatic depressurization system would also be condensed in the pressure-suppression pool, and this pool serves as one source of water for the emergency core cooling system. Systems for the control of combustible gases from metal-water reactions and radiolytic decomposition of the water are also provided to assure that flammable concentrations are not reached in the containment.

The secondary containment system is the reactor building which houses the reactor and its primary containment system; a typical on-line system appears in Fig. 8. The buildings, substructures, and exterior walls up to a level above the top of the drywell are of poured-in-place reinforced concrete. The secondary containment of operating BWR plants is designed for low leakage, and has sealed joints and interlocked double door entries. Under postulated accident conditions, the normal building ventilation system would automatically shut down and the building would be exhaust-ventilated (so as to maintain a slight negative pressure therein) by two parallel standby systems which discharge through the plant stack or roof exhaust system, to minimize ground-level exfiltration possibilities. These gas treatment systems include high- efficiency particulate air filters and solid absorbents for trapping radioactive halogens, particularly iodine, that might have leaked from the primary containment.

\section{Safety considerations}

As discussed above, it is not possible for a light-water reactor to explode like a nuclear bomb. The unique aspect of a nuclear power plant is the accumulation of a large amount of radioactivity. The central issue in lightwater nuclear reactor safety is the prevention of a major release and dispersal to the environment of this radioactivity.

\section{Accumulated radioactivity-amount and location}

The total amount of stored radioactivity in a nuclear power plant depends upon the duration and power level of its operation. When a light-water nuclear power plant in the 1000 MWe size is first placed into operation, it is loaded with unirradiated, but naturally radioactive fuel (enriched to about $3 \%{ }^{235} \mathrm{U}$ ) having an agg regate activity of about 150 curies in a typical PWR loading and about 300 curies in a BWR loading. Table VI lists some typical PWR and BWR fuel loadings and discharge data for reference.

This fresh fuel is only slightly radioactive; however, during reactor operation, the fission process produces 
large amounts of radioactivity. If the reactor is operated at full power, the amount of radioactivity increases to the order of $1.7 \times 10^{10}$ curies between refueling operations, which occur about once a year. The largest fraction of this radioactivity is due to the fragment radioisotopes produced by the fissioning of the uranium, and is contained in the fuel rods. Another fraction is due to neutron capture in ${ }^{238} \mathrm{U}$ to form ${ }^{239} \mathrm{U}$ and other radioactive actinides. Finally, a lesser amount of radioactivity is induced in the reactor structural material and in the primary coolant by the absorption of neutrons produced in the fission process. Since only a fraction, one-third to one-quarter, of the core is replaced during refueling, a large inventory of radioactive material is retained in the reactor core after initial power operations (see Table VI).

When the reactor is shut down, the generation of radioactivity ceases, and the quantity of radioactivity in the spent fuel decreases, initially at a very rapid rate due mostly to the decay of short-lived fission products. This radioactivity continues to generate substantial quantities of heat in the spent fuel. This heat production initially amounts to some $225 \mathrm{MWt}$ in a $1000 \mathrm{MWe}$ reactor but decreases with time as radioactive decay progresses.

Table VII provides some calculated values for the quantities of radioactivity and heat associated with an 1100 MWe PWR after a sustained period of operation. The table indicates how these quantities would decrease with time after reactor shutdown. To a good approximation, these values would also apply to a comparable BWR core under the same circumstances.

\section{Prevention of release and dispersal of radioactivity}

During normal operation, the public is protected from the release of large amounts of radioactivity at three levels.

i. Nearly all radioactivity $(>98 \%)$ is contained inside the uranium dioxide fuel pellet matrix which is itself encapsulated as rods in zircaloy cladding. ii. The fuel rods that make up the reactor core are sealed in the primary coolant system, the system that removes heat from the reactor core.

iii. A containment structure houses the reactor coolant system and is designed to confine and remove any radioactive material that escapes from the primary system.

The only known way that a large fraction of the radioactivity in the fuel rods can be released is to melt the uranium fuel pellets. (The zircaloy cladding would fail before the pellets melt.) It is, therefore, crucial to maintain adequate cooling to prevent melting of the fuel core, since, if the core does not melt, most of the radioactivity will remain confined.

\section{The roles of various organizations involved in assuring safety in the nuclear power industry}

Several different organizations, with quite distinct roles and responsibilities, work together in the implementation of nuclear power. We will summarize these considerations for four organizations: power utilities, architect-engineering firms, reactor system vendors, and the AEC. ${ }^{8}$ Our aim is to provide the reader with important background needed to understand how reactor safety is actually implemented.

It is important to recognize that the safe design, construction, and operation of a proposed plant is the responsibility of the licensee (i.e., the utility). The AEC's responsibility is to ensure that the licensee and his contractors take all necessary steps to protect the health and safety of the public. Both responsibilities extend throughout the life of the plant, from the time that initial planning begins to the time the plant is ultimately decommissioned. The degree of expertise concerning nuclear power varies widely among U. S. electrical utilities. Some have built and operated several power reactors and have professional staffs who have familiarity with the

TABLE VII. Calculated radioactivity of 1100 MWe PWR ${ }^{a}$ at shutdown and as a function of decay time. ${ }^{b}$

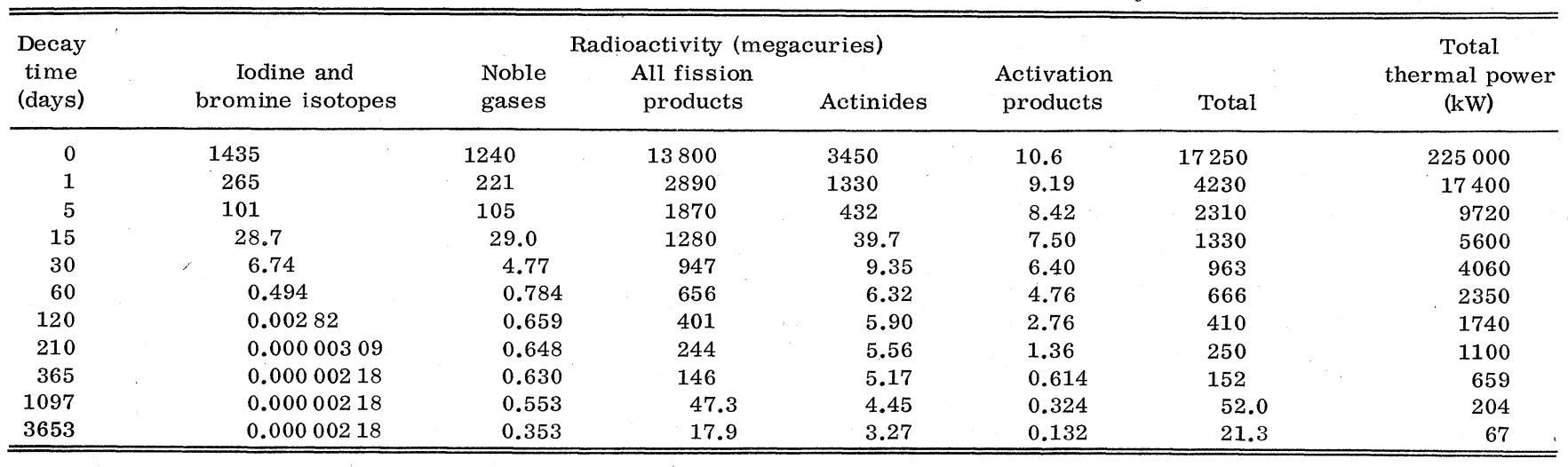

${ }^{\text {a }}$ Reactor is assumed to be shut down just before refueling after a sustained (293-day) period at a specific power of $37.5 \mathrm{MW} /$ metric ton. The time average specific power over the previous 1100 days is $30 \mathrm{MW} / \mathrm{metric}$ ton. The reactor is fueled with $3.3 \%$ enriched uranium totaling 82 metric tons of enriched uranium fuel.

b Table from Report WASH-1250.

${ }^{8}$ See Footnote 1. 
technology; in other cases, especially with smaller utilities, there may be minimal experience. In any case, the utility usually hires one of several well-recognized architect-engineering firms, who are experienced at carrying out the detailed selection, design, and planning for the site, the various buildings, and the many subsystems to suit the needs of a particular utility.

The architect-engineer must keep the AEC Regulatory staff informed during this early period, so that the AEC is cognizant of the plans as they develop, and so that a wide variety of AEC regulations can be satisfied. Thus, while the initiative and ultimate responsibility for safety rest with the utility, the architect-engineering firm normally plays a crucial role in the design process under the overall safety umbrella furnished by the procedures of the AEC Office of Regulation.

Once the utility's plans have undergone AEC review and approval, construction can begin. The nuclear part of the power station is manufactured by one of four U. S. light-water reactor vendors: Babcock \& Wilcox, Combustion Engineering, General Electric, and Westinghouse (a fifth manufacturer, General Atomic, sells hightemperature gas-cooled reactors). Each vendor firm has been in the business since the early days of commercial nuclear power and carries out its own extensive analysis and product development efforts, consistent with a philosophy that has emphasized strong commercial participation. In the process of convincing the AEC Office of Regulation that a license is justified, the vendor in question submits a "Preliminary Safety Analysis Report," containing many safety analyses and calculations to support his application for a safe design that satisfies the AEC regulations. The vendor will have analyzed a wide variety of hypothetical accident situations, as well as many aspects of the local site that bear on safety questions. The plans for quality assurance programs also receive attention. The AEC Regulatory staff performs an independent evaluation of the main points presented by the architect-engineer and the reactor vendor, a process that generally takes at least a year, often more, even though the architect-engineer and reactor vendor follow very specific methods of regulation and documentation laid down by the AEC. During construction and installation there are also extensive inspection procedures and practices in which the AEC satisfies itself that the various buildings and power reactor subsystems are being constructed and installed according to plan. It is quite common for discrepancies and minor problems to arise and be corrected during this period, which typically lasts five or more years.

When reactor construction is completed, the utility applies for an operating license, submits a "Final Safety Analysis Report," and another set of procedures and regulations is enforced. The AEC must be satisfied that the utility has an adequate and properly trained staff, with plans to maintain its quality, and that there are adequate plans to maintain quality assurance of all the components during their lifetime. When the AEC is satisfied and the many required inspections have been completed, the fuel may be loaded and officially licensed operation may be$\mathrm{g}$ in. Again, it is common during the initial shakedown period to uncover and correct minor problems, occasionally even major ones which were unforeseen or which might arise because of parts found to be defective or inadequate. In any case, an AEC inspector watches over the situation and ascertains that all regulations have been satisfied and that full operation can be approved. The AEC continues to carry out periodic inspections of both hardware and procedures to ascertain that the utility, which bears formal responsibility, is maintaining safe operation.

It can be seen that there are many organizational interfaces involved in order that private initiative can be stimulated and, at the same time, controlled through regulation intended to ensure public safety. While the AEC provides the overall safety umbrella in the form of licensing and regulatory procedures and inspectors, it is no simple task for the utility and its principal contracting organizations to assume the initiative and to bear the ultimate responsibility for safe operation. Many safety issues rest on how effectively the process is carried out by all parties. 


\section{INITIATING EVENTS}

In this report we have focused on events leading to a loss of core cooling followed by failure of the containment and the subsequent consequences. This chapter is concerned with possible causes of a loss of the normal core cooling for an operating nuclear reactor. Section $\mathrm{A}$ is a discussion of the integrity of the primary cooling system. Section B deals with certain events other than rupture of the primary system which might lead to a loss of cooling. Section C discusses the importance of good quality assurance in maintaining high system reliability against the failures outlined in Secs. $\mathrm{A}$ and B. Finally we consider the possible safety problems brought about by human intervention: through either operator error (Sec. D) or sabotage (Sec. E).

\section{A. Primary system integrity}

The integrity of the primary system is of central importance to the safety of the reactor, since breaks in the primary system could result in loss of cooling. An accident of this sort is called a LOCA (loss-of-coolant accident), and may range in severity from a catastrophic rupture of the reactor pressure vessel (almost certain to lead to core melt) to rather minor leaks in pipes, which could almost surely be handled by the engineered safety features of the reactor.

The most serious event by far, considered virtually inconceivable by the AEC and the industry, would be a catastrophic rupture of the reactor pressure vessel, below core level. Such a rupture would leave no defense against core melt-down, in that cooling water could not be contained near the core. The pressure vessel is made of steel, normally six to twelve inches thick, roughly a dozen feet in diameter, and three to four times as high. It is made of carefully chosen material, meticulously welded, and inspected both before and during operation. The question of the extent to which this care can provide assurance against pressure vessel rupture is the subject of this section, along with some discussion of pipes.

Reactor pressure vessels and primary piping are designed to conform to American Society of Mechanical Engineers (ASME) standards, which are cautiously evolutionary developments of other standards appropriate to non-nuclear boilers and piping. For this reason we will discuss non-nuclear experience below, as an indicator of the efficacy of these standards. We believe the standards to be sound, but they must be backed by meticulous conformance at every stage of materials preparation, fabrication, and testing.

All pressure vessels belonging to light-water cooled reactors made in the United States are currently made of a particular steel (A 533B) which has been selected because of its ductility and resistance to brittle fracture at the operating temperatures of the reactor. The weld materials are similarly chosen, the combination specifically designed to provide a material with as high a "fracture toughness" as is possible, according to current understanding of the mechanism of fracture.

The understanding of fracture mechanisms has advanced considerably in recent years. In the case of brittle fracture, crack growth is governed by the balance of the extra energy required to create new surfaces and the stress relief energy released through the relaxation of the material. When the former is larger the crack does not grow, while it will if the latter is dominant. Since the two energies scale differently, there is a minimum crack size for rapid growth, and any crack larger than this size will cause disruptive failure of the material at a given stress level. Similar phenomena appear in the more complicated situations in which part of the material is undergoing bulk plastic deformation while the rest of it is involved in linear elastic behavior, and criteria have been developed for determining minimum crack size from basic material parameters determined by standardized tests. The genealogy of a disruptive crack would be: (1) an inevitable small crack due to manufacturing imperfections; (2) a period of slow growth determined by fatigue, cycling effects, corrosion, etc.; (3) a final disruptive failure when the crack reaches critical size for the conditions of the material and the stress situation at that time.

The steel used for reactor pressure vessels has relatively brittle behavior at low temperatures $\left(100^{\circ} \mathrm{F}\right.$ or less), but is extremely ductile and tough at the reactor operating temperatures $\left(500-600^{\circ} \mathrm{F}\right)$. The technical specifications for nuclear plant operation require a careful track from a cold start to operating pressure and temperature, so that the vessel will not be stressed when it is below ductile temperatures during startup. The critical crack size at operating temperature is of the order of inches, a size that ought to be detectable by the test procedures to be discussed below.

All of these considerations presuppose that the materials of the vessel and the piping possess the specified fracture toughness; that the stresses do not exceed those for which the system is designed; and that inspection procedures ensure that embrittlement of the materials through fatigue, radiation, and corrosion is held to a safe level.

Failures could follow from departures of materials or loadings from specifications, either through changes in the materials as a result of use or misuse, or as a consequence of overloading. Clearly an inspection and maintenance program is important in assuring the continued high quality of the pressure vessel and piping.

During and after fabrication, reactor vessels are subjected to ultrasonic and radiographic testing which now has enough sensitivity in principle to detect flaws of a size that might threaten catastrophic rupture of the vessel. In addition, an overpressure test serves as a double check on the vessel integrity. The problem is, therefore, to maintain a monitoring and testing program dur ing the life of the reactor of sufficient intensity and persistence to guarantee that the main embrittling mechanisms (i.e., radiation, fatigue, and corrosion) will not compromise the system. Such a program is required by Sec. XI of the ASME Code.

Current steels for reactor pressure vessels are rather insensitive to radiation embrittlement, if attention is paid to limiting the concentration of certain trace impurities such as copper. It is estimated that no substantial embrittlement will occur during the assumed 40-year life of the pressure vessel. In addition, samples 
of the materials are kept in the pressure vessel in regions which receive larger neutron irradiation than the most heavily irradiated portion of the vessel itself, and are removed for analysis from time to time during the life of the vessel. It can therefore be anticipated that embrittlement of the test specimens is certain to occur before the vessel itself is compromised, and the likelihood of unexpected radiation embrittlement is small.

Fatigue embrittlement of the primary system piping is much more difficult to estimate, depending as it does on the fluctuations which are the normal accompaniments of regular operation of the reactor. We think it important that these fluctuations be quantified, along with their likely effect on the primary system materials. We simply do not have the information available to make a judgment on the importance of these effects, nor can we judge the importance of corrosion embrittlement, of which there have been some instances.

In view of the remaining uncertainties, it is important that continued attention be devoted to the development of improved radiographic and ultrasonic testing techniques, to reduce further the likelihood that a critical flaw may escape detection. These inspections have recently been extended to the more LOCA-sensitive parts of the primary system piping. In addition, the acoustic emissions technique for monitoring slow crack growth, though still unproven, should receive continued development.

One point which needs mention, especially since much was made of it during the recent British controversy over the selection of a nuclear electric system, is the question of "leak-before-break." In relatively thinwalled pressure vessels it may well be the case that the critical flaw size for disruptive failure is more than the thickness of the vessel itself, so that one can have substantial confidence that the vessel will display a noticeable leak before major failure occurs. Indeed, the experience with thin-walled pressure vessels in non-nuclear service bears out this presumption. In the extremely thick vessels used in nuclear service, however, the critical flaw size is likely to be rather less than the thickness of the vessel, so that the possibility of an embedded flaw large enough to cause failure, but not yet large enough to cause a leak, cannot be ignored. For such flaws, the nondestructive inspection techniques mentioned above must be entirely trustworthy, and meticulously and redundantly implemented. What uneasiness we have about primary system integrity derives from the concern that, if there are 10 or 20 years without any major reactor accidents, inspection and maintenance procedures may be allowed to relax.

In the end we have to ask whether boiler vessels and piping do in fact fail in normal service. Unfortunately, nuclear experience is not sufficient to provide much guidance on the issue, since only just over a hundred reactor years of U.S. commercial LWR history has been accumulated. The U.S. Navy experience with smaller systems is approximately ten times that, but it is not known to us whether naval designs, procedures, and experience are relevant to large commercial LWRs. However, if one were to include naval experience, one could say that there have been no major violations of primary system integrity in roughly a thousand reactor years of experience. Even this is simply not enough information on which to base a sanguine prognosis.

A number of organizations have therefore undertaken to collect boiler and piping statistics from non-nuclear sources, recognizing that the codes under which these vessels were built and operated are somewhat less demanding than those for nuclear components. Nonetheless, the data are expected to provide something of an upper limit on failure rates to be expected in nuclear experience, and are valuable in that regard. There have been some 750000 vessel years of non-nuclear experience, with a disputed number of relevant failures varying from zero to two or three (the definition of relevance is at issue here) (AEC, WASH-1285, 1974). For those vessels, these statistics indicate that the likelihood of unexpected disruptive failure is of the order of, or less than, one part in a few hundred thousand per vessel year. The Advisory Committee on Reactor Safeguards (AEC, WASH -1285, 1974) has added another factor of ten for the higher quality and maintenance standards in nuclear plants.

For all piping smaller than 6 inches in diameter, a reasonable estimate of the chances of a LOCA would be a factor of 10 higher, but the consequences of a failure in such a pipe are mitigated by the engineered safety features of the reactor, in particular the emergency core cooling system, discussed below.

Special mention must be made of the tubes in the steam generator of a PWR, because these are present in very large numbers, are a part of the primary system (comprising, in fact, $50 \%$ to $75 \%$ of the surface area of the primary system), and have historically been subjected to corrosive failure. Under normal operating conditions, it is possible to sustain the loss of a few tubes in the steam generator without a major threat to the integrity of the reactor, but the loss of large numbers could constitute a serious problem. Various measures are now being implemented to reduce the rate at which these tubes fail. On the other hand, behavior of the tubing in the steam generator under accident conditions, especially if partly corroded, is an uncertainty.

Finally, though this is a discussion of primary system integrity, it is important to note that the integrity of the secondary system in a PWR is also of great importance. The secondary system is normally responsible for ultimately disposing of heat from the reactor, and a multiplicity of independent secondary coolant loops is standard design for a PWR.

In summary, we believe that assurance of the integrity of the primary system of a commercial LWR is best guaranteed by continuous and meticulous attention to inspection, maintenance, and operation. There are still unknowns in the basic theory of crack growth, and the program to resolve these will be discussed below (see Sec. VI-Reactor Safety Research Program). Nonetheless, given the meticulous engineering, design, and quality assurance that are required, we believe that the integrity of the pressure vessel is one of the strong points in reactor safety. It is clear, however, that further work toward better methods for nondestructive testing and in situ monitoring of the primary system would strengthen the quality assurance and inspection programs considerably, a point that will be amplified in our review of the safety research program. 


\section{B. Transients}

During the course of routine reactor operation, departures from normal operating conditions are expected to occur, and these are called anticipated transients. Obviously, there may also be unanticipated transients. Examples of transients may be: reactivity changes caused by inadvertent control rod withdrawal, cold water injection from an inactive coolant loop, etc.; equipment failures such as failure of a main feed water pump, failure of a recirculating pump, turbine trip, secondary coolant loop failure in a PWR, etc.; loss of off-site or control power, fire; and so forth.

The reactor control system can automatically adjust for the majority of transients without service interruption, but some will require rapid termination of the chain reaction, or SCRAM, for safety reasons. SCRAM itself is not normally a major event and occurs in the average reactor about ten times a year; but the sequence of events from an anticipated transient to a safe shutdown of the reactor involves the performance of a number of systems whose failure could lead to serious problems, including loss of cooling.

The primary requirement in bringing about a safe shutdown is to maintain an adequate supply of coolant to the core. This requires among other things that there are no pressure transients severe enough to cause a rupture in the primary coolant system, and that there be an adequate heat sink to dispose of decay heat. Failure to provide such a heat sink would lead to the boiling out of primary coolant through the pressure relief valves and would result in a core melt, typically in a couple of hours. It is important that the system be reliable, redundant, and invulnerable to those common mode failures which we can foresee.

It is not always necessary to bring the reactor all the way to the cold shutdown condition in the event of a transient, but, if it is, the essential steps are the cessation of the chain reaction (SCRAM or poisoning of the coolant water ${ }^{9}$ ) and the removal of decay heat from the primary system for a sufficient time. The AEC has recognized certain transients as "anticipated" since they occur about ten times a year in the average reactor. The AEC estimates the probability of SCRAM failure as $10^{-7}$. Assuming that these transients do not affect the SCRAM capability in any way, the probability of transients without SCRAM is thus calculated as about $10^{-6} /$ reactor year (AEC, WASH-1270, 1973). The industry is required to show that the reactor can survive a transient with SCRAM failure, assuming that nothing else fails. Studies are currently under way at the vendors and at Brookhaven National Laboratory to investigate the question.

Removal of decay heat requires the availability and mobility of coolant, as well as adequate heat sinking. While electrically powered pumps are normally used in a PWR to move the coolant, it is important to note that

\footnotetext{
${ }^{9}$ In the event of a loss-of-coolant accident, the boiling away of the primary coolant in the initial stages of the accident also stops the chain reaction, since the water is the moderator for the reactor.
}

convective transfer is sufficient within the primary system to transfer decay heat to the secondary system. Thus, in the event of complete electrical failure in a PWR, for example, convective cooling would be suf ficient to transfer the decay heat from the primary coolant to the secondary coolant until the latter boiled away. To get a feel, therefore, for the safety systems provided against a severe transient, we will discuss in somewhat more detail the cooling systems and their electrical requirements, for both a PWR and a BWR.

In a PWR in normal operation, the secondary coolant loop consists of the main feedwater pump which is driven by either steam or electricity, the condenser, and the electric condensate pumps. In the event of failure of this system, there is an auxiliary feedwater system, normally consisting of one steam-driven pump, which is driven by steam from the reactor in the event of massive electrical failure, and two electric pumps which are on the emergency power bus. Any one of these pumps is sufficient for decay heat removal. The normal water inventory for this system is sufficient for approximately eight hours of operation, and it can be manually replenished from much larger supplies. Failure of this system would occur only after an extended loss of ac power, together with the failure of the auxiliary feedwater system steam pump.

For a BWR the normal cooling loop consists of the main feedwater pump, the condenser, and the condensate pumps. If the condenser is not available as a heat sink, then heat in some BWRs is channeled through the drywell to the torus, which has the heat capacity to absorb about one day of decay heat. It is in turn cooled by the high-pressure service water system, which operates from the emergency ac bus, and such cooling can continue indefinitely.

The above illustrates the importance of maintaining an electrical supply to the reactor (even though one can get by for a while with complete loss of power), and for this reason there is redundancy in the electrical system.

The emergency system consists of two completely independent power trains, either of which is sufficient to ensure proper operation. All safety-related equipment is duplicated, one element being fed from the first pow er train and the other from the second. Physical separation and redundancy are required. Control power for the instrumentation is derived from duplicated dc buses, which are normally fed by rectified ac. In addition there are backup batteries floating on the buses, which are sufficient to supply the control needs of the station for approximately six hours, although this varies considerably from plant to plant. Loss of dc power could lead to very serious consequences, since it would result in many control functions being lost.

If both sources of off-site power fail, emergency ac power for the plant is provided by auxiliary diesel generators, whose arrangement varies from plant to plant. Some two-unit plants have as many as six diesel generators, one assigned to each emergency train in each unit, and backups in the event of failure of one of the other four.

Surprisingly, diesel performance is one of the recognized weak points in the safety system: statistics show 
that about $3 \%$ of diesels fail to start when asked (AEC/O0E, 1974a). In addition, there is a finite probability (approximately 1\%) that a diesel, properly started, may trip when asked to assume full emergency load. Clearly, improvements in diesel reliability are necessary, since off-site power failures do occur, and the AEC appears to be moving in the direction of more stringent requirements on diesel backup systems.

If there were an extended loss of off-site power and diesel failure, which could result in a core melt, there might well be a large radioactive release as the containment spray systems, heat removal system in a BWR, etc. would not function. Further, if the batteries run down, control systems would be lost.

It is extremely difficult to estimate the probability that some sort of transient will cause a core melt. It is much easier to analyze a LOCA caused by a break in a defective pipe, when the reactor is otherwise operating essentially normally. Here the pipe failure is the dominant event and the probability of core melt depends mainly on this single failure rate. In addition, the sequences themselves are not as complicated, since other equipment is presumably largely unaffected.

Some classification of transient-initiated accidents is possible, and there are approaches which we recommend be explored. For example, a large class of transients could lead to both the loss of the heat sink for the primary coolant, and the loss of ECCS. This would result in the boil-off of the primary coolant in a period of hours. In this class are fires and massive electrical failures. The remedy here might be to provide an alternate heat sink, such that convective circulation is sufficient to remove decay heat. It might well be possible to provide a secondary heat exchanger to a passive, manually actuated auxiliary coolant system, made large enough to remove decay heat. If this could be done economically, it would protect against a large class of transients, whose likelihood might be significant but is extremely difficult to estimate.

Since 1969 the AEC has been actively working on another class of transient accident, the "anticipated transient without SCRAM." Work is actively under way to complete the analyses of failure to SCRAM, and preliminary indications are favorable.

There are transient accidents which fall outside of these two classes, in particular transients involving operator error. It is difficult to be certain that one has been able to identify and protect against all possible transients. Although it is possible to improve the independence of engineered safety features, transient accidents remain a serious concern.

\section{Quality assurance}

In the study of the primary system integrity and transients, we recognized that the safe operation of a reactor depends heavily upon maintaining the high quality of components and the high reliability of reactor systems. Quality assurance (QA) encompasses those procedures necessary to assure that the specifications written into the design and operating plans of a facility are met in practice, throughout the sequence from design to fabrication, testing, operation, and maintenance. It is the role of the AEC Office of Regulation to ensure that QA procedures are adequate to protect the public. This role is important but difficult because large commercial reactors are among the most complex devices ever built, and it is not possible for even a trained expert to inspect such a facility fully. The difficulty will be exacerbated by the tremendous growth anticipated in the nuclear industry, since the AEC will need more and more inspectors but will be competing for qualified people with the reactor industry itself. It is our opinion that possible future problems of QA may be an important limitation on safety in the nuclear power industry, especially if we are lulled into complacency by a long period of safe operation of reactors.

Two issues must be addressed: (1) what is the adequacy of the present level of QA, and (2) what should be done to guarantee a persistently high level during the planned expansion of the industry? For the latter issue, we can do little more than underline the potential problem. For the former, we have found that, to our knowledge, no objective and quantitative measurements of the present QA system's effectiveness exist, even though there are a number of possible ways to go about making such measurements. We strongly recommend that such an objective measurement program be instituted, and below we suggest some forms that such a program might take.

Because of widespread understanding of the importance of QA, the nuclear industry has a multifaceted QA program, designed to assure a reliably high level of human performance. The AEC in turn, through its regulations, attempts to insure that each sector of the nuclear industry maintains adequate QA procedures. In the AEC philosophy there are four levels of QA: (1) Each person has the responsibility to assure the quality of his own work. (2) The organization in which the individual works has the responsibility to assure his quality of workmanship through management, inspection, training programs, and supervision. (3) The ultimate user of a piece of equipment bears the responsibility for its quality (usually, this means the utility which owns and operates the plant). The utility has responsibility not only for its own QA program, but also for the QA programs of its suppliers, contractors, and subcontractors. For example, the utility must assure itself that the QA program of each manufacturer of important components is satisfactory, must take ultimate responsibility for QA failures of the architectural and engineering firm that constructs much of the plant, and must incorporate a description of the reactor vendor's QA program as a part of its Safety Analysis Report. (4) The AEC, through its Regulatory staff, has an inspectorate charged with the responsibility of making the entire system work well. The AEC philosophy places the AEC inspectors more in the role of administrative auditors than of physical inspectors. An AEC inspector, upon visiting a site on either an announced or unannounced inspection, typically examines a representative sample of the QA records kept by the utility. These records provide the written documentation of each inspection and QA check performed, and include reports of all abnormal situations found. Besides this inspection of records, the AEC inspector performs certain physical 
inspections himself, usually in a random spot-check approach but with special attention to areas which have given recent trouble. For example, the instructions for a surprise inspection might include the following: "Inspect any four of the control room panel voltmeters, including among the four at least two which required repair or recalibration within the past three months (if at least two such exist)." Such physical inspections are supplemented by interviews, some specified and some randomly chosen, with various utility personnel at different levels of responsibility.

This system will work well if there are few errors with safety significance, and if the licensee's program uncovers and corrects these few errors with sufficiently high probability. If both of these conditions were not met, assurance of public safety would require a far more thorough AEC inspection. It is clearly important to have a quantitative objective measure of how frequently significant errors now escape detection. In fact, it is necessary that the poorest licensee QA program acceptable to the AEC be good enough to protect the public safety at some reasonable level of risk. There are many sources of information that can cast some light on this question.

(1) The AEC auditing and spot checking program finds errors from time to time (the majority of which do not have significant safety implications). There is information in these data which, if compiled and analyzed well, might enable a quantification of how well the present QA system is working.

(2) The licensees report abnormal occurrences as they happen. It may be possible to make a more thorough analysis of these occurrences.

(3) Measurements of the error detection efficiency of the inspection staff, obtained by double inspection procedures, could provide indications of the probability that a significant error escapes detection.

(4) The individuals who work within the QA system should know how well it is working, although such information is hard to quantify.

(5) Study of specific incidents may be a source of information which could not be uncovered in statistical studies.

(6) Studies of comparable or related systems can provide insight into how well the nuclear QA system should be expected to function, and may help to indicate whether significant improvements can reasonably be expected. Examples include nuclear QA programs in other countries, as well as non-nuclear QA programs of comparable complexity.

Unfortunately, none of these various approaches to quantification is developed sufficiently to enable us to pass quantitative judgment. Therefore, what we have to say rests upon our own qualitative judgments, combined with input from individuals who are in the system. Such inputs are not hard to come by. They cover the entire range from full confidence to expressions of serious doubt, a range which is in itself troublesome to us. This may mean that QA performance is nonquantifiable; it surely means that it has not yet been quantified in a way which is understood in the QA community.
Our approach has been to analyze some of the data available on QA failures, including the summaries of abnormal occurrences reported to the AEC, a few specific incidents in which QA failure has played a role, and informal information from AEC staff members and consultants. We have also considered the analysis in the draft of the recent AEC Reactor Safety Study (WASH 1400 ), in which potential QA failures seem to play a significant role in some important accident sequences. It is clear to us that the present QA program misses at least a few errors per year which have actual or potential safety significance, but it is not at all clear whether it is necessary, prudent, or even possible to improve the system significantly. We simply do not know whether the undetected errors really represent the bare minimum, given human frailty; whether they are important; or whether the expenditures necessary to eliminate them might not be more fruitfully spent in other safety-related areas. However, we do believe that the efficiency is in some measure quantifiable, and we strongly recommend that measures be taken toward this end, both from the analysis of experience already reported and from new measurements on the QA system.

The quantification of QA performance, using one or more of the methods we have outlined above, has some specific, identifiable benefits. The most important is that better data will be available concerning whether the present system is now sufficient to protect the public safety, and whether the quality of the QA program is maintained as time passes. Another important benefit is that it provides a basis for the more efficient use of available QA resources, by revealing which areas are weak and which are strong. Still a nother is that quantification enables more direct comparisons among QA programs at different reactor installations.

Among the more promising approaches to quantification are more thorough analyses of data which now exist or are being taken now but for other reasons; and a program of partial double inspection by the AEC. In such a double inspection program, two inspectors would independently inspect the same items at the same installation in succession but without communication. At a level of about $10 \%$ of the total of all inspections, this might go far to determine how well the present QA sys tem works. If done in a controlled way, such a double inspection process might fulfill the basic need under discussion here, by determining in a statistical sense to what extent significant errors may truly escape detection, thus calibrating the last level of QA. To our knowledge, there is now no such double inspection program within the AEC.

In summary, we feel that attention to QA issues is important because lapses in QA may be important contributors to the overall risk to the public from nuclear reactors. We have not been able to find quantitative measurements of how well the present QA program is working, and this concerns us, because without such measurements there is no good way to keep tabs on whether the overall QA program is (and will continue in the future to be) adequate for safety. We have suggested a number of possible approaches to obtaining better information, among them a double inspection program and better analyses of the data which now exist. 


\section{Operator error}

In studying the compilations of abnormal occurrences reported to the AEC by nuclear licensees, it is apparent that a significant number of them were either caused or aggravated by operator errors. For example, of the 861 such abnormal occurrences contained in the AEC compilation for 1973 (AEC/O0E, 1974b), 132 (about 15\%) were attributed to "personnel error." Of course, most of these incidents seem to have had little or no significance for safety, but a few had either potential or direct safety significance. Also, analysis of a few of the more significant incidents reveals that operators frequently make errors of both commission and omission (AEC/ 00E, 1972). From these and other data we conclude that from time to time the mistakes made by operators can have safety significance.

We limit this discussion to Reactor Operators and Senior Reactor Operators, both of which are well-defined positions requiring licensing under AEC regulations. A prospective operator must undergo extensive training prior to being licensed; a license must be renewed every two years; and recently the AEC has imposed the requirement that each reactor licensee (utility) establish a requalification program (lectures, operational training, and written examination) in order to maintain and upgrade the competence of its operators. These requalification programs, whose technical content is generally of high level, appear to have been designed more for upgrading than for mere enforcement, a philosophy which seems appropriate to us.

When we inquired into the overall quality of operators, we found a consensus in the industry that the training, level of overall competence, and motivation of the operator staffs are excellent, and that their training is appropriate for the job. This general feeling of confidence seems to be widespread. We have not come to an independent judgment on this issue.

How is the high community opinion of the operators to be reconciled with the fact that operator error contributes to a significant fraction of the abnormal occurrences? Two important effects help to account for the operator errors. The first is a general inadequacy in human engineering of the control room and control consoles, and the second is that in emergency situations there are limits on what can reasonably be expected of operators.

On the human engineering issue, we concluded, during visits to several nuclear plants, that the control consoles were generally inadequate compared to similar consoles familiar to several of us (accelerator control rooms, air traffic control rooms, etc.). This point has also been noted in WASH-1400 Draft:

\footnotetext{
"In general, the design of controls and displays and their arrangements on operator panels... deviate from human engineering standards specified for the design of man-machine systems and accepted as standard practice for military systems." (Appendix III, p. 136.)
}

WASH-1400 Draft also notes that the quality of written instructions provided to operators is low.

The other important observation is that in emergency situations, one should not expect consistently high performance from operator crews. This observation is the result of several classic studies on human performance in emergencies, and should come as no surprise to anyone familiar with reactions of individuals under high pressure or in severe emergencies.

Considering the vast number of possible accident situations, it is clear that neither training nor written manual procedures can anticipate them all. Hence, some operator intervention based on decisions in real time must necessarily be allowed for. Yet we have concluded that the typical reactor control room has certainly not been automated to the extent desirable. The reasons are many and even conflicting. Perhaps the foremost is historical, since acceptance of control sequence automation of routine functions has always been a slow process. Another consideration is that manual control of a certain number of parameters keeps the operators alert and allows them to feel that they are indeed operating the reactor. Finally, it is argued that automatic systems may be less reliable than manual systems, may be more costly to install and maintain, and may be more difficult to license. A good overview of problems with automation has been given by Epler and Oakes (1973).

We feel that increased control sequence automation will have significant impact not only on safety but also on plant reliability, which has its own indirect safety implications. Our confidence is based in part on recent studies of the subject within the nuclear control community itself, for example as reviewed in the recent "Symposium on Power Plant Dynamics, Control, and Testing” (ANS, 1973).

Discussion of this point is now widespread in the community, and the American Nuclear Society has sponsored a committee whose charter is to study computer usage in the control of nuclear power plants and to recommend a standard for the design of such computer systems, if appropriate. This group has considered the full range of possible modes of automation, from nearly complete computer control of emergency functions to the use of computers in a purely advisory (data-presentation) capacity. As of late 1974 the committee has not yet completed its work. One difficulty which has been recognized by the committee as contributing to slow acceptance of computer usage is that the licensing of such systems requires a demonstration that they are at least as "safe" as the existing systems... a demonstration which is essential but difficult. We recommend that this issue be given prompt, high-priority attention in all sectors of the industry. There seems to be inadequate incentive for automation, possibly because of the added capital expense but also because of perceived difficulties in getting such systems approved for licensing. One reactor vendor who has offered an automated data-presentation system as part of its control room package for some time has had only limited success in convincing utilities to buy this option.

A very important part of operator training and retraining is the use of simulators. Within the past few years, the number of control-console simulators in use has expanded greatly, and their role in operator training has been strongly endorsed by the AEC (Code of Federal 
Regulations, 10 CFR 55, Appendix A). We applaud this trend. Unfortunately, only recently has much thought been given to programming these simulator consoles for a variety of abnormal, accident-type situations. These accident-simulator programs, which we endorse and encourage, could serve not only the purpose of operator training, but three other purposes as well: to help the industry to understand whether or not actual consoles are human-engineered to enable operators to cope with accidents; to provide a training bench for control-sequence automation programs, by testing them against simulated accidents; and to provide some data (albeit an upper bound) on how well operators respond in various accident situations.

In summary, we recommend improvements in the human engineering of reactor consoles, encourage further control-sequence automation, and suggest that there be a more vigorous use of simulators for training operators to cope with abnormal and even accident conditions.

\section{E. Sabotage}

It is conceivable that a group of people might threaten or carry out sabotage of a nuclear power plant in such a way as to cause great damage and loss of life. Potential reservoirs of radioactivity that might be released by acts of sabotage include liquid and gaseous waste storage facilities, spent fuel, and the reactor core itself. We see no way to estimate the probability that such acts might be attempted either for coercive or outright terrorist purposes, but it is possible to estimate credible ranges of possible damage, in terms of fatalities, illnesses, and property denial and decontamination costs. It is also possible to a nalyze specific plants and existing or hypothetical plant physical security measures to determine their vulnerability to sabotage attempts of various kinds.

It is difficult to conceive of acts of sabotage at a nuclear plant that could kill substantially more people or cause more property damage than would result from the worst type of accident that could be caused by equipment failure-i.e., a core meltdown soon after shutdown of the reactor, accompanied by failure of the containment structure. Nevertheless, we have seen no thorough and realistic analyses designed to establish credible ranges of damage (in terms of immediate fatalities, latent cancers, short-and long-term denial of the use of property, decontamination costs, and possible effects of public panic) from successful sabotage at specific nuclear power plants located near densely populated areas under the worst credible weather conditions. The estimate of approximately 2000 fatalities and 5 billion dollars in property damage resulting from "worst case" accidents presented in Draft WASH-1400, for example, is an average for several reactors close to especially densely populated areas, under the "worst" weather conditions for those areas. Even this estimate, furthermore, may underestimate the number of delayed casualties and the property damage for the case that was considered (See Sec. V of this report).

We are also unable to judge whether or not reactor sabotage, under the worst conditions, would present a more severe threat to society than some other conceivable types of nonmilitary acts of violence that might be accomplished by non-nuclear means (e.g., large-scale, systematic arson in a densely populated area; dispersal of chemical or biological poisons in enclosed areas or water supplies).

It is nevertheless imprudent, we believe, to assume that there is negligible probability that an inadequately protected nuclear power plant might be used as means for purposeful acts or threats of large-scale violence. Furthermore, it is possible to conceive of circumstances in which the use of explosions, for example, to disable a nuclear power plant, without an explicit intention to release dangerous amounts of radioactivity, might actually result in a significant hazard to the public. If such an event occurred in a foreign country where acts of terrorism are fairly common, this might stimulate similar action in the United States, either for terrorist or coercive purposes.

In the course of our study, we considered several possible ways to cause intentionally a LWR core meltdown and containment rupture. A subgroup of our working group was given a classified briefing by an AEC contractor that had arrived at some preliminary results of a study of sabotage of a specific LWR power plant. Based on our findings we recommend that detailed studies be carried out to determine the consequences of various acts of sabotage at specific power plants near heavily populated areas, the costs and effectiveness of countermeasures designed to prevent such acts, and the effectiveness of alternative contingency plans for reaction to such acts or threatened acts. Although the AEC has recently sponsored studies of this subject, we believe that more detailed studies are likely to suggest further actions that would reduce the vulnerability of nuclear plants to sabotage, at reasonable cost and without excessive interference with normal plant operations. In carrying out such studies, we suggest that particular attention be given to

- means to assure that the reactor is shut down before unauthorized people can gain control of any area immediately adjoining any item of vital equipment.

-high confidence methods of communication to off site law enforcement control centers immediately following any indications of either unauthorized entry into the plant or unauthorized actions in the vicinity of items of vital equipment.

-detailed analyses of the range of credible off-site consequences (for specific sites) of actions designed to cause core meltdown, as a function of the time after shutdown at which the actions are accomplished.

-determination not only of the direct costs of additional countermeasures but also of their effects on normal plant operations and on-site safety.

-assessment of the use of protective security personnel who are federal employees and have full law-enforcement authority, rather than personnel provided by private security services or armed plant employees who do not normally have such authority.

-determination of the total costs of plant security systems as functions of their overall effectiveness as measured either by the reduction in the probability that 
sabotage could take place or by the reduction in the maximum consequences of sabotage.

- involvement in the studies of people with extensive professional experience in the fields of physical security protection and sabotage-counter sabotage operations.
Preliminary looks at several possible improvements of current physical security at nuclear power plants indicate to us that significant counter-measures to sabotage could be implemented at reasonable cost and without interfering with normal operations. 


\section{COURSE OF AN ACCIDENT}

The preceding discussions focused on events and causes that could lead to a loss of the normal core cooling for an operating nuclear reactor. This portion of the report is concerned with what happens if there is a loss of normal cooling. Analyses have indicated that a large break in the primary system piping resulting in a loss of coolant could have large potential radiological consequences to the public. We will discuss the development of such an accident, beginning with a discussion of how such an accident might progress with an effective emergency core cooling system (ECCS).

The ECCS is intended to prevent serious damage to the reactor in the event of a loss-of-coolant accident (LOCA), and all operating power reactors are equipped with them. If the ECCS were to function adequately, fuel cladding temperatures would be maintained well below the melting point. Although some fuel swelling and rupturing may take place (with consequent release of stored gaseous and some volatile fission products), the reactor core will be maintained in a "coolable geometry" so that massive core distortion will not take place. In principle, brittle failure of the fuel rods during the quenching process, as the rods are cooled in the hypothetical accident, would also be prevented by successful ECCS operation. The ECCS is also designed to provide the long-term cooling necessary to remove the slowly decaying energy production of the reactor's fission product inventory for as long a period as is necessary following the LOCA.

As part of its reactor licensing and evaluation responsibilities, the AEC has formulated a set of "Acceptance Criteria" which specify minimum acceptable standards for ECCS performance. In the absence of completely definitive test procedures for system performance, these criteria specify methods by which the analysis and evaluation of ECCS function must be performed. The criteria prescribe required and acceptable features of evaluation models which are used to calculate the cooling performance of the ECCS. We will discuss and qualitatively evaluate the Acceptance Criteria; review several methods for quantifying the evaluation of their adequacy; and discuss briefly the possibility that alternate or modified ECCS concepts may result in greater confidence in the adequacy of emergency core cooling system performance.

We will discuss containment behavior for LOCAs with and without effective emergency core cooling, and investigate the potential radiological consequences to the public if the containment should fail. This includes an analysis of one of the more severe reference PWR accidents treated by Draft WASH-1400. Ways in which the consequences of a large release of radioactivity could be mitigated will be suggested in conclusion.

\section{A. Description of a loss of coolant accident (LOCA)}

As discussed in Sec. III, the LOCA is the principal design basis accident against which the effectiveness of the ECCS is evaluated. Although the ECCS is intended to cope with a wide range of possible breaks in the primary system piping, most attention has focused on the sudden severance of the large diameter cold leg pipe for the PWR or of the recirculation jet pump inlet pipe for the
BWR. Postulated breaks of these lines have generally been found to impose the most severe demands on ECCS performance for both types of reactors.

In evaluating ECCS performance during a LOCA, the Acceptance Criteria prescribe that the reactor is assumed to be operating at $102 \%$ of full power before the hypothetical accident, thus including a marg in for such factors as instrument error. The temperature of the zircaloy cladding at this time would be near that of the adjacent water, in the neighborhood of $600^{\circ} \mathrm{F}$. The average temperature of the hottest $\mathrm{UO}_{2}$ pellet would be well above $2000^{\circ} \mathrm{F}$ with a peak temperature in the center greater than $4000^{\circ} \mathrm{F}$. The excess heat content of the $\mathrm{UO}_{2}$ at this average temperature, called the "stored heat," is approximately proportional to the power density and is affected by the thermal resistance of the "gap" between the $\mathrm{UO}_{2}$ pellets and the cladding. The stored heat is important because it would contribute significantly to the early temperature history of the cladding, in the event of a LOCA.

Since the reactor system response, and hence ECCS requirements, would be fundamentally different for small breaks than for large ones, we will describe both LOCA phenomena separately below.

\section{Small LOCA}

If a small break or leak were to develop, sensors would note the drop in reactor system pressure and automatically cause (i) the chain reaction to be shut down rapidly by SCRAM and more slowly through the addition of poisoning boron compounds to the coolant and (ii) the operation of high-pressure injection systems (discussed in Section III) which inject coolant at or near the actual reactor operating pressure. The fuel in the core would then cool down as the stored heat of the reactor core, primary system internal energy, and a decreasing amount of fission product decay heat were removed by the flow of the coolant as driven by either pumps or natural convection.

\section{Large LOCA}

LOCAs could range in size up to a break of the largest pipe in the primary system (which may be approximately one meter in diameter). As the primary design basis accident by which the adequacy of the engineered safety features (especially the ECCS) of each plant is measured, the AEC has chosen the hypothetical severance of the largest pipe in the primary system. The break is assumed to occur instantaneously, in such a way that the reactor coolant would discharge unimpeded from both ends of the severed pipe. This type of break is referred to as a "double-ended guillotine break." The sequence of events describing the core history in a large LOCA of this type with effective emergency core cooling can be divided into four periods: blowdown, refill, reflood, and long-term cooling. ${ }^{10}$ This assumed sequence is de-

\footnotetext{
${ }^{10}$ See Appendix I for a more complete description of a LOCA with an effective ECCS.
} 
scribed in more detail below. The successful resolution of a large LOCA, from pipe break until control of the initial thermal transient is achieved and long term cooling begins, might take as long as 3 to 5 minutes. Of that time period, blowdown (the period of rapid pressure decay and coolant expulsion in the reactor) might take from 20 to 30 seconds in a PWR, or as long as 30 to 60 seconds in a BWR. Peak temperatures during reflooding of the reactor core would probably occur 1 to 2 minutes after a LOCA initiation for both PWRs and BWRs. Following termination of the initial temperature transient, long-term cooling would be required for an indefinite period, until active recovery procedures were instituted for the plant facilities.

\section{Period 1: Blowdown}

Following the hypothetical severance of the large cold leg pipe, there would be a violent pressure release, or blowdown, during which most of the water originally in the reactor pressure vessel would be expelled into the containment structure. Very early in the LOCA, the prompt fission heating would stop as the density of the water moderator decreased. At the same time, the cooling of the outside surface of the zircaloy cladding would diminish sharply because of the altered hydrodynamic flow. Under these conditions, the temperature variation across the uranium dioxide and the zircaloy due to the stored energy of the fuel would tend to even out, dropping the peak temperature at the center of the $\mathrm{UO}_{2}$ and increasing the temperature near the $\mathrm{UO}_{2}$ surface and the zircaloy cladding. If there were no heat removal from the outer surface of the zircaloy, its temperature would quickly approach the average temperature of the $\mathrm{UO}_{2}$, approximately $2000^{\circ} \mathrm{F}$. Fortunately an appreciable amount of the stored heat would be removed during blowdown by the rushing water and steam as they escape from the reactor vessel, thus limiting the initial rise in temperature of the zircaloy. Even so, estimates made with approved ECCS evaluation models of peak temperature during blowdown run as high as $1700^{\circ} \mathrm{F}$.

In addition to stored heat, the fuel pellets would continue to be heated by the radioactive decay of the fission products and of the short-lived actinide elements that were produced during reactor operation. At shutdown, decay heat amounts to approximately $7 \%$ of the rated thermal output of the plant, but decreases rather rapidly for a short time thereafter. Thus, a plant with an electrical capacity of $1000 \mathrm{MW}$-with consequent thermal capacity of about $3300 \mathrm{MW}$-would produce about $230 \mathrm{MW}$ which must be dissipated immediately after shutdown. Assuming plant operation prior to the LOCA at or near its rated capacity for at least a period of several months, the decay heat output would decrease to $5 \%$ of rated thermal output 10 seconds after shutdown, drop to $2 \%$ after 10 minutes, and reach $1 \%$ after 2.25 hours. Within 24 hours after shutdown, the decay heat would fall to about $0.5 \%$ of the rated thermal output of the plant.

Though the decay heat would be a substantial contribution to the energy which must be dissipated during the initial thermal transient of the LOCA, it is well to keep in perspective the relative magnitudes of the stored heat and decay heat released during this period. Stored heat released during the first minute of a large LOCA might be equal to, or greater than (by as much as factors of 2 to 4 ), the energy generated by the decay heat during the same period. Moreover, if the zircaloy cladding reached temperatures of about $1800^{\circ} \mathrm{F}$ or above, additional energy would be released through the reaction of the zircaloy with steam to form hydrogen and zirconium dioxide. For cladding temperatures of $2000^{\circ} \mathrm{F}$, or greater, the energy released from this metal-water reaction could also be of the same order of magnitude as the decay heat-at least for periods of the order of one minute. These heat sources would cause the average temperature of the fuel rods to start to increase after the cooling effect of blowdown ceased, with the temperature of the zircaloy keeping pace with that of the fuel pellets.

As the pressure decreased in the ruptured nuclear steam supply system during blowdown, ECC fluid would be injected into the reactor in order to replace the lost coolant. ECC coolant would generally be injected into the cold legs of a PWR, or directly into the pressure vessel head above the core for a BWR (see figures in the Primer, Sec. III). In the most severe LOCA scenarios, all of the emergency core coolant that would be injected into the reactor during blowdown is assumed to be entrained by escaping steam and liquid (expelled during flow reversal through the core) and carried directly out the break without ever collecting in the bottom of the pressure vessel.

Hydraulic forces that would result from the blowdown are not expected to be significantly disruptive to the core. However, the greater potential for disruptive changes in the core geometry during blowdown would be associated with possible swelling and rupture of the fuel rods. During fabrication, fuel rods are pressurized with helium to approximately the operating pressures of the reactors. During normal reactor operation, gaseous and volatile fission products are generated and retained within the fuel rods, increasing internal pressures. If the fuel rod cladding becomes too hot during the LOCA, the internal pressures would exceed the yield stresses of the zircaloy and localized fuel rod swelling would occur. If temperatures got above $1400^{\circ} \mathrm{F}$ during blowdown, substantial swelling and rupture of the cladding would be expected.

\section{Period 2: Refill}

Refill is defined as the period following blowdown after the flow of fluid though the break is negligible. During refill, the ECC fluid would no longer be expected to bypass the core-as it might during blowdown-but would begin to fill the primary pressure vessel. The refill period would continue until the coolant reached the bottom of the core. Fuel rod cooling during this period would occur only as a result of natural convective currents in the steam-filled core; and consequently cooling during refill would be the poorest of any period in the LOCA.

As a result of the poor cooling capacity during the refill period, core temperatures at the hottest portion of the core would begin to rise, because of decay heat, at 
rates of approximately $15^{\circ}-20^{\circ} \mathrm{F} /$ second for PWRs and $9^{\circ}-12^{\circ} \mathrm{F} /$ second for BWRs. If they started from $1500^{\circ} \mathrm{F}$, local core regions would reach temperatures greater than $2000^{\circ} \mathrm{F}$ within 30 to 60 seconds. At these temperatures, the zircaloy-steam reaction begins to become a significant energy source. It is therefore important that the ECCS begin cooling the core as rapidly as possible, with reflooding being initiated before such elevated temperatures are reached.

\section{Period 3: Reflood}

Reflooding is defined to begin when the ECC fluid reaches the bottom of the core. The temperature excursion would eventually be terminated during this period as fluid refills the core. At the beginning of the LOCA, the reactor's power-producing chain reaction would be shut down by SCRAM and/or the loss of the coolant-moderator. During reflood, steps would be taken to assure that the chain reaction would not be reinitiated. SCRAM, which would normally already have occurred, would provide such insurance and neutron absorbing poisons (such as boron) would also be injected along with the emergency coolant.

Both PWRs and BWRs have ECC systems in which water would reflood the reactor. In BWRs the reflood would be provided by water from the low-pressure injection system and the core spray system. In a successful reflood, the rate at which the emergency cooling water filled the core (the reflood rate) would have to be high enough to remove the decay heat and heat produced by zircaloy oxidation. In order to give confidence in ECCS performance, the calculated reflood rate should clearly have a substantial margin over the rate that is just sufficient to turn the temperature excursion around in a short time.

As the cooling water reached the hot core, much of it would be converted to steam; and it is this steam together with entrained water droplets that would provide the initial cooling of the hotter regions of the core. For the reflood water to continue entering the core, it must displace the steam, which would have to escape from the reactor vessel and find its way into the containment atmosphere. In the pressurized-water reactors, part of the steam would have to flow through the steam generators and pumps to escape through a cold leg break. The reduction of the reflood rate by the relatively high resistance to flow in this path is called "steam binding." Steam binding is a potentially serious threat to adequate reflood rates. The phenomenon will be discussed at greater length below.

Boiling-water reactors (BWR) would not be subject to steam binding, because their system design provides a more direct path for the steam to escape, but the same requirement for rapid reflooding would have to be met if excessive cladding damage were to be avoided. Boiling-water reactors do have a core spray system that would start about 30 seconds after the occurrence of the break, but its cooling effect on the central rods of a fuel bundle might be insufficient, in itself, to prevent the fuel from exceeding acceptable temperature limits. Without the support of the low-pressure injection system, the performance adequacy of the ECCS would be in serious doubt for the BWRs.

As the thermal transient terminated and the fuel rods began to reach temperatures at which quenching could occur (from about $700^{\circ}$ to $1000^{\circ} \mathrm{F}$ ), the fuel rods would undergo very large stresses. At this time, if the metalwater reaction had induced significant oxidation of the zircaloy cladding, brittle failure of the fuel rods might occur. Such failures migh release fuel pellets and cladding fragments which could block coolant passages and reduce the cooling hazardously, if the failures occurred in significant numbers.

\section{Period 4: Long-term cooling}

In this period cooling would be maintained to ensure the long-term dissipation of the decay heat.

\section{B. ECCS analysis and evaluation methods-Acceptance Criteria}

On June 29, 1971, after a review by the AEC Regulatory Staff and the Advisory Committee on Reactor Safeguards established that the current procedures for evaluating ECCS performance were of uncertain adequacy, the AEC published a set of "Interim Acceptance Criteria" establishing minimum standards for analyzing and evaluating all ECCS designs used by reactor manufacturers. These criteria were the subject of lengthy public rulemaking hearings during 1972 and 1973. The hearings required a total of 125 days of oral testimony and generated over 22000 pages of transcript and thousands of pages of written testimony and exhibits. Participating in the hearings were the AEC Regulatory Staff, all four U.S. LWR manufacturers, a consolidated group of electric utilities, and the Consolidated National Intervenors-a group of about 60 organizations and individuals whose principal technical representatives were Henry W. Kendall and Daniel F. Ford. As a consequence of the hearings, a revised set of Acceptance Criteria ${ }^{11}$ were promulgated by the AEC on December 28, 1973. These criteria, supported by a lengthy and detailed statement of the opinion of the Commission, were substantially more conservative and comprehensive than the Interim Acceptance Criteria.

\section{Acceptance Criteria elements}

The Acceptance Criteria are quite general in nature, specifying only five items. These five criteria elements, which are calculated consequences of the most serious LOCA in which the ECCS is assumed to function, are:

(1) Peak Cladding Temperature - the calculated maximum fuel element temperature must not exceed $2200^{\circ} \mathrm{F}$.

(2) Maximum Cladding Oxidation-in order to limit oxidation-induced fuel rod embrittlement, the calculated equivalent stoichiometric oxidation of the zirconium cladding for the hottest rod shall not exceed $17 \%$ of the

\footnotetext{
${ }^{11}$ The Acceptance Criteria have been reproduced in full in Appendix III.
} 
total cladding thickness before oxidation.

(3) Maximum Hydrogen Generation-the calculated total amount of hydrogen generated from the chemical reaction of the zirconium cladding with water or steam throughout the entire reactor must not exceed $1 \%$ of the total amount which could possibly be generated if all of the metal in the fuel cladding were to react.

(4) Coolable Geometry - all calculated changes in the core geometry shall leave the core amenable to cooling.

(5) Long-Term Cooling-assuming successful initial operation of the ECCS, the system shall be capable of maintaining calculated core temperatures at acceptably low levels by removing the long-lived fission product decay heat remaining in the core over whatever extended periods of time are required.

\section{Criteria-specified evaluation models}

As is readily apparent, the above criteria prescribe only the calculated system response to a LOCA. Consequently, the criteria also include by specification " Required and Acceptable Features of the Evaluation Models." These elements of the criteria provide more detailed enumeration of the features of the calculational codes which must be used for ECCS performance evaluation. Only a summary outline of this portion of the criteria will be included here as Table VIII. A complete listing of the Acceptance Criteria is presented as Appendix III. The outline given in Table VIII does show, however, the kind of detail necessary to provide the required standards for the adequacy of the calculational procedures.

The complexity of modeling LOCA response/ECCS performance will be discussed in more detail below. However, it should be noted that the principal problem of the criteria formulators has been to assure that the overall system response has been conservatively predicted. Many elements of the evaluation models are uncertain and no large-scale system tests exist against which to evaluate overall system modeling; consequently the AEC has attempted to prescribe each of the discrete parts of the evaluation models conservatively. The implicit assumption behind this endeavor is that if all parts of the evaluation models are prescribed conservatively, then the resulting calculated system performance must also be conservatively prescribed. If this assumption is valid, it could clearly lead to over-designing of the system. However, the complexity of the evaluation models and the ranges of uncertainty in their elements make it extremely difficult to provide a conclusive demonstration of the validity of this vital assumption. In fact, though many reactor design experts are qualitatively satisfied that ECCS performance will be adequate, a quantitative evaluation of the conservatism of the ECCS performance evaluation methods may be nearly unachievable, as we will discuss below.

\section{ECCS evaluation}

In this section we shall discuss the nominal adequacy of the ECCS to meet the apparent short- and long-term heat removal requirement for reactors. We shall also present a qualitative evaluation of the relative conservatism of the elements of the Acceptance Criteria. Quan- titative evaluation of the performance adequacy of the ECCS proved to be beyond our scope (and beyond that of all others who have examined the question to date). However, we shall discuss the several methods which might be used to perform such an evaluation. Finally, we shall discuss the possibility of development of alternate or modified ECCS concepts which might improve ECCS performance.

\section{Nominal adequacy of ECCS design elements}

Physical descriptions of the basic elements of the PWR and BWR ECCS have been presented in Sec. III. We shall not repeat that description here except to note that care has been taken to assure that ECCS fluid delivery systems for all power reactors of current design are quite

TABLE VIII. Required and acceptable features of the evaluation models.

I. Detailed Specifications are Given for the Following Model Parameters. ${ }^{\text {a }}$

A. Sources of heat during the LOCA

1. Initial stored energy in the fuel

2. Fission heat

3. Decay of actinides

4. Fission product decay heat

5. Metal-water reaction rate

6. Reactor internals heat transfer

7. PWR primary-to-secondary heat transfer

B. Swelling and rupture of the cladding and fuel rod thermal parameters

C. Blowdown phenomena

1. Break characteristics and flow a. Spectrum of pipe breaks

b. Discharge model (Moody)

c. End of blowdown (PWRs only)

d. Noding near break and ECCS injection points

2. Frictional pressure drops

3. Momentum equation

4. Critical heat flux (CHF)

5. Post-CHF heat transfer correlations

6. Pump modeling

7. Core flow distribution during blowdown

D. Post-blowdown phenomena; heat removal by the ECCS

1. Single failure criterion

2. Containment pressure

3. Calculation of reflood rate for PWRs

4. Steam interaction with ECC water in PWRS

5. Refill and reflood heat transfer for PWRs

6. Convective heat transfer coefficients for BWR fuel rods under spray cooling

7. BWR channel box under spray cooling

II. Required Documentation

Detailed and Specific Documentation of Each Reactor Manufacturer's Evaluation Model is Required under the Criteria, Including:

1. Description (with a complete computer program listing)

2. Solution convergence demonstration

3. Sensitivity study performance requirements

4. Comparisons of model predictions with applicable experimental information required

5. Specification of "General Standards for Acceptability" of models

\footnotetext{
${ }^{a}$ See Appendix III for a complete reproduction of the acceptance criteria.
} 
redundant. For example, PWRs have not only separate pump-driven high- and low-pressure injection systems, but also have a passive pressurized accumulator fluid delivery system. All are designed to meet varying needs for the spectrum of LOCAs which must be considered in ECCS design. Similarly the BWR has not only high- and low-pressure core spray systems, but also has a third large capacity low-pressure coolant injection system. The independence and redundancy of these systems are very beneficial design attributes.

Similarly, the nominal capacity of the pump-driven low-pressure injection systems for both PWRs and BWRs has been adequately sized to meet the demands of longterm decay heat removal for the reactors. For example, the PWR low-pressure injection systems have sufficient capacity to deliver a flow of $1 \%$ to $5 \%$ of normal coolant flow. As discussed in the I.OCA description, such flow capacity in the ECCS should be completely adequate within a few seconds after shutdown. Similarly, the combined outputs of the BWR low-pressure core spray and injection systems provide a coolant flow of $3 \%$ to $12 \%$ of that normal for a BWR. Moreover, both BWR and PWR low-pressure systems are supplied by several pumps on independently wired electrical circuits so that even if one or more should fail, satisfactory long-term operation of the system can be maintained. Therefore it appears that long-term steady-state capacity of the systems has been well and conservatively designed. Moreover, the designs provide sufficient redundancy in the system so that single failures of ECCS pumps will not cause the entire system to break down.

\section{Evaluation of Acceptance Criteria elements}

Thus the more critical questions are associated with the adequacy of the phenomenological treatment of the elements of the Acceptance Criteria, for which we shall present a qualitative evaluation of some of the more important elements.

The sequential events of the critical LOCA phenomena have been discussed briefly above and are presented in more detail in Appendix I. An outline of the more significant elements of LOCA phenomena is presented in Table IX, along with qualitative estimates of the conservatism of corresponding elements of the ECCS Acceptance Criteria. Again, a more detailed discussion of the conservatism of the criteria is presented in Appendix I.

\section{a. Energy sources}

The energy sources during the LOCA, as outlined in Table IX, are of critical importance to the coupled thermo-hydrodynamics of the reactor response to emergency core cooling. As noted, methods for the treatment of each individual element of the energy sources are prescribed in the ECCS Acceptance Criteria in at least a moderately conservative fashion. Although the prescription of the individual elements of the energy sources may not be strongly conservative when considered separately, when taken as a whole the conservatism of all the energy source elements appears to be adequate.
Only one reservation exists in our minds with respect to the treatment of LOCA energy sources in the ECCS Acceptance Criteria. The potential for LOCA-induced failure of steam generator tubes is not covered by the criteria. Based upon evidence of substantial steam generator tube corrosion with frequent consequent leaking under normal operating conditions for most PWRs (Babcock \& Wilcox reactors appear to be exceptional cases with few or no steam generator tube corrosion problems), it appears that steam generator tube failure during the LOCA is an event of significant probability. Failure to consider steam injected from the PWR secondary into the primary reactor coolant loop is potentially a very serious weakness in the overall conservatism of the specification of energy sources in the Acceptance Criteria.

\section{b. Structure behavior}

Specifications for methods of analyzing the structural response of most elements of the nuclear supply systems are not included in the ECCS Acceptance Criteria. Nevertheless, calculations for the design of the structural response of the system during a LOCA are apparently regularly and carefully conducted. However, it seems that the omission of structural response criteria is an oversight which may be nonconservative. Specification of criteria in this area would be desirable.

Two special cases of structural behavior are included in the Criteria which should be discussed: (1) those pertaining to fuel rod cladding brittle failure due to thermal shock during reflood and (2) massive core migration and pressure vessel melt-through in the event of fuel melting due to ECCS failure.

The ECCS Acceptance Criteria specifications of peak cladding temperature limits of $2200^{\circ} \mathrm{F}$ and metal-water reactions limits of $17 \%$ equivalent stoichiometric oxidation have been specifically designed to preclude thermalshock-induced brittle failure as a cladding failure mechanism. However, the experimental results upon which the criteria are based do not entirely assure ductile response of the cladding when subject to the limiting temperature-oxidation conditions. The results suggest, instead, that the cladding may be left in a partially ductile condition for which brittle failure during quench cannot be precluded. Thus there may be a possibility of fuel rod failure from this mechanis $m$ which could contribute to impaired cooling.

However, assuming nominal power peaking factors (local power distribution relationships) within the core, rods which might reach the $2200^{\circ} \mathrm{F}$ criteria limit are likely to be localized to a relatively small region of the core-from $5 \%$ to $15 \%$ of the total cross-sectional area of the core. If cladding exposure is limited to within criteria levels of less than $17 \%$ equivalent zircaloy oxidation, then massive melting and core geometry changes resulting from brittle failure of fuel rods appear unlikely. Since fuel pellets are likely to be relatively cool at the time brittle failure might occur (on the order of $1000^{\circ} \mathrm{F}$ ), relatively small amounts of material would be likely to be dispersed when cladding failure occurred. Consequently, additional mechanical damage induced 
through melting in the core should be relatively light, and coolability would probably not be seriously perturbed.

\section{c. Coolant fluid dynamics and core thermal behavior}

Of the remaining parameters affecting the core thermal response, i.e., the heat transfer characteristics and coolant fluid dynamics, the latter is the more uncertain. The ECCS Acceptance Criteria specification of heat transfer parameters during blowdown and refill periods appears to be adequately conservative, though data bases for postcritical heat flux heat transfer, swelling and rupture of cladding and fuel, and cladding thermal properties need improvement. (AEC programs

TABLE IX. Critical LOCA phenomenological behavior and conservatism of related ECCS criteria treatment. Criteria adequacy in relevant ILOCA period.

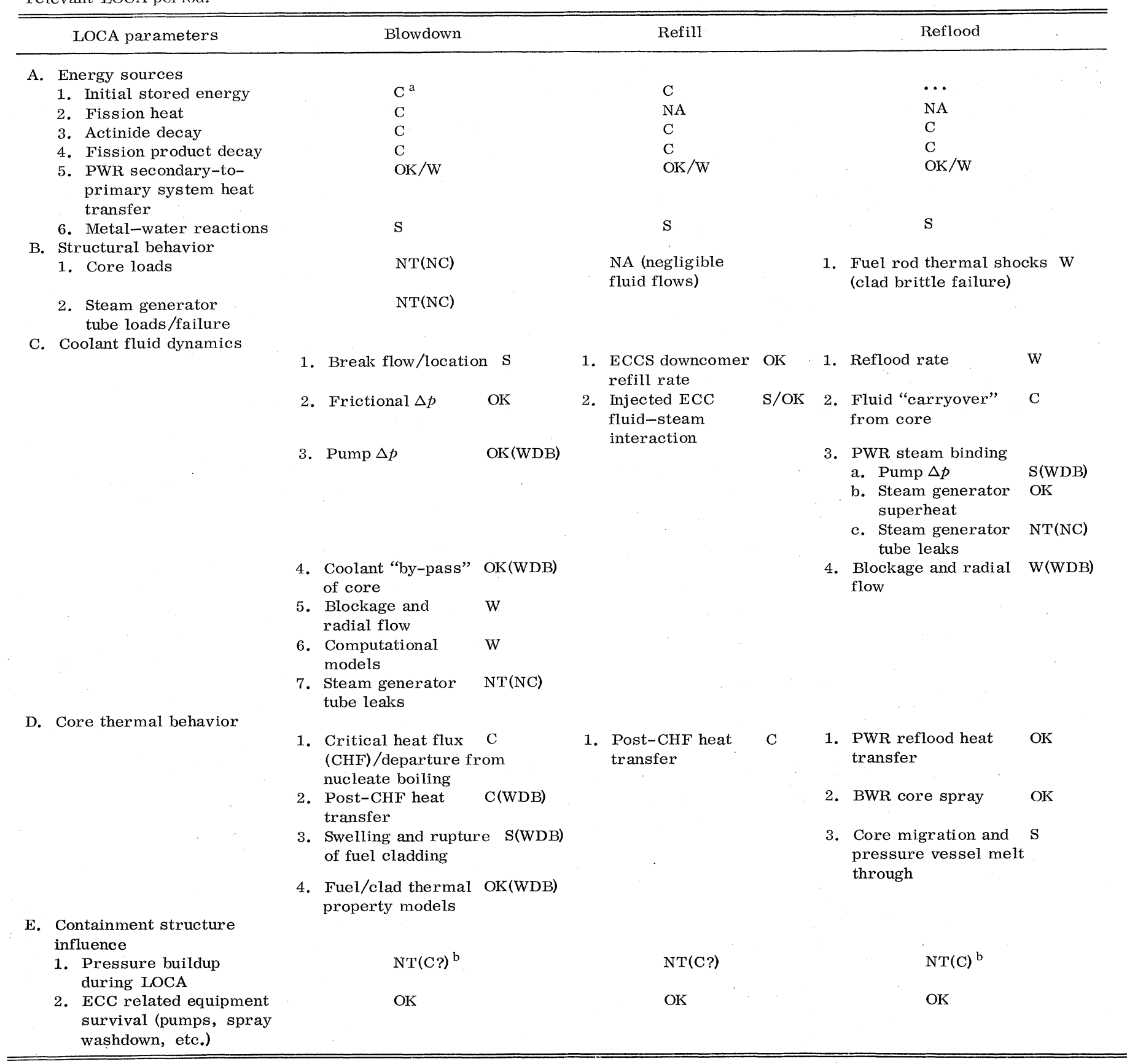

a Definition of symbols: S, strongly conservative; C, conservative; OK, adequate or "realistic;" W, weak; NT(NC) not treated in criteria-nonconservative omission; NA, not applicable; WDB, weak data base.

b Definition of symbols: NT(C?), not treated in criteria-conservatism of omission uncertain; NT(C), not treated in criteriaomission is conservative. 
are underway to provide better definitions of these parameters.)

Heat transfer during reflood has, however, been defined empirically in the criteria in terms of the results of two experimental programs: the BWR and PWR Full Length Emergency Cooling Heat Transfer (FLECHT) tests. These extensive, but controversial, test series are being utilized, in accordance with ECCS Acceptance Criteria specifications, in a manner which probably gives results which are closer to mean values of the data than they are to a clearly conservative interpretation of the data. Consequently heat transfer coefficients in the very important reflood period can only be considered adequate and not truly conservative.

Uncertainty in the conservatism of heat transfer coefficients is exacerbated by even greater questions about the conservatism of estimates of the quantities of fluid flowing through the core throughout the LOCA, but especially during reflood. No adequate empirical data exist by which the validity of predicted reflooding rates can be established. The validity of predictions of reflood rates depends entirely upon the adequacy of the calculational methods by which the results are obtained. It is generally agreed that the current generation of calculational methods for ECCS fluid dynamics needs substantial improvement. Consequently the conservatism of predicted fluid flow rates throughout the LOCA, and especially during the critical reflood period, must be considered uncertain.

\section{d. Steam binding}

As previously mentioned, the very real potential for steam binding in the conventional sense, exacerbated by steam generator tube leaks in PWRs, may seriously compound the heat transfer problem during the LOCA. It appears that rupture of a few (on the order of one to ten) tubes releasing secondary steam into the depressurized side of the reactor could increase the steam binding pressure barrier against the injection of emergency cooling water and could severely limit the rate at which reflooding can occur in a PWR. It should be noted that if a substantial number of steam generator tubes were to rupture, the large resulting flow of steam from the secondary side of the reactor might actually aid cooling. However, it appears more probable that, if there were any steam generator tube failures at all, they would occur in relatively small numbers. Consequently, with the present PWR designs, adequate cooling may not be possible in the event of steam generator tube failure during a severe LOCA.

A unique feature of the BWR-ECCS is that all elements of the ECC system inject their fluid directly into the reactor pressure vessel above the core. This feature of BWR design-together with the reduced complexity for primary system steam flow (no steam generators are used in BWR designs)-essentially eliminates steam binding as a problem for BWRs.

\section{Methods of evaluating ECCS performance adequacy}

On the basis of the above discussions of the Acceptance Criteria and the qualitative evaluation of their elements, it is clear that the specified evaluation models are regarded as a key element in evaluating ECCS performance. The need for high confidence in predicting the performance of the ECCS has led the AEC to require use of evaluation models which they feel are adequately conservative. Two major questions are raised by this specification. First, is the AEC's definition of "adequate" or "substantial" conservatism acceptable? Second, can a quantitative value be determined for the degree of conservatism of ECCS performance, as is customary in the design of many other pieces of equipment where public safety is involved?

If the degree of conservatism of the ECCS codes is to be established (assuming they are indeed conservative), then codes which predict the LOCA phenomena "realistically" must also be available for comparison. Unfortunately, the theoretical evidence for the adequacy of either realistic or conservative models is poor. Moreover, the experimental work which is needed to test the adequacy of the models has not yet been completed (and perhaps has not even been planned). Consequently, the quantitative evidence for evaluating ECCS code adequacy is not available.

In this section we will discuss methods by which the quantitative evidence might be obtained. We will begin by discussing the basis for "realistic" modeling methods and comparing them with evaluation models. The need for analyses of the sensitivity of the codes to the various system parameters, as a mechanism for investigating model conservatism, and for test programs to provide quantitative measurements of code adequacy will be discussed.

\section{a. Computer codes}

Because of the great complexity of phenomena involved in a LOCA, it is necessary to incorporate the various models of these phenomena into computer codes in order to calculate the dynamic fluid and thermal behavior for the reactor system. There will be some discussion below of the validity of these codes, in terms of the adequacy of their predictions of ECCS performance. However, it should be remembered that the ECCS codes are not really three-dimensional hydrodynamic calculations of the flow of two-fluid systems in complicated geometries. There is a major program underway for the improvement of these codes, and the following is addressed to their current and near-current status.

The magnitude and complexity of attempting to describe a LOCA in rigorous detail throughout a reactor makes it clearly out of the question to do conventional hydrodynamics by prescribing a flow field consisting of a defined velocity, density, temperature, at each point in the reactor and then connecting them with the differential equations familiar from the study of hydrodynamics in other contexts. This is a three-dimensional flow problem, involving two phases of matter (gas and liquid), which would instantly exhaust the potential of the largest computers. The compromise that is made in ECCS codes is to use a lumped parameter approach to describe the system in which the reactor is "hodalized" by dividing it into volumes with a prescribed connectivity. Such 
a description is, of course, a very simplified model of the flow in a reactor, and the extent to which one thereby simulates the flow is dependent to some extent upon the complexity of the nodalization. Further, some features such as the behavior of pumps must in the end be treated empirically. Similarly, the jet flow from an orifice is modeled according to a theoretical upper limit to the flow. Also, the development of two-phase system models is really just in its infancy.

In this relatively simple approach to system modeling, the integrated conservation equations for each nodalized element of the reactor are obtained by utilizing the usual stream tube approximation for fluid flow, which assumes that geometry changes are gradual. Gradual geometry changes are, of course, not the case in a real reactor. Consequently, the credibility of applying the stream tube approximation must rest upon an extensive program of testing these simplified codes against a wide variety of experimental flow situations resembling the real situation. The degree to which the credibility of this and other code approximations is being established is a function of the adequacy of the AEC research program, which will be discussed in greater detail in Sec. VI.

In any case, it is important to recognize that no computer code now exists which will adequately describe the detailed flow field in a reactor in abnormal circumstances. This description, if it is to come in the foreseeable future, must be derived from a sensitive and interactive parallel development of code and experiment, the latter providing the framework in which one can resolve the hydrodynamic problems into components that are tractable. Such success is not to be expected in the very near future, but is not out of the question.

\section{b. Experimental support to code development}

For PWRs, some attempts have been made to demonstrate the validity of "realistic" codes and the conservatism of "evaluation models" against experiments conducted on the so called "Semiscale" apparatus at the Idaho National Eng ineering Laboratory. These experiments with a relatively small-scale (of order $1 / 1000$ in power), electrically heated model of a PWR are discussed in more detail in connection with the AEC reactor safety research program presented in Sec. VI. However, several of the experiments have been selected as "Standard Problems" against which to demonstrate the validity of the AEC and manufacturers ECCS performance codes. To date, correlations of calculations and experimental results for the "Standard Problems" have been poor. They have not provided the anticipatedand sought for-demonstration of the general adequacy of LOCA/ECCS numerical methods. These and other problems have caused some people, both within and outside the AEC, to question the credibility of the current generation of codes. ${ }^{12}$

An extensive program is being sponsored by the AEC to overcome the lack of quantitative evidence for evaluation model adequacy through a new and improved generation of LOCA computational methods and an extensive experimental program. However, the current experimental program appears to be heavily aimed at resolving the problems on a "separate-effects" basis, rather than with systems analysis.

The LOFT program described in more detail in the discussion of the reactor safety research program (Sec. VI) is the only major U.S. experimental systems-effects investigation being conducted (or even planned) to integrate all the elements of the system. Though LOFT is clearly an important program, its scale is small in comparison to PWRs of current design (about $1 / 60$ th power). In Sec. VI, we will discuss the ability of LOFT to provide answers to the questions of ECCS adequacy. It will be seen that we consider it unlikely that the AEC program, as presently conceived, will lead to an assured quantitative demonstration of the ECCS safety margin.

\section{c. Parametric sensitivity studies}

The AEC agrees that without adequate system test results, systematic parametric studies, or codes adequate to validate the conservatism of the ECCS designs, no adequate quantitative basis exists at this time for evaluating the marg in of conservatism for reactor performance under LOCA conditions (AEC/REG. 1973, p. 140). In the absence of quantitative experimental support, a complementary approach for evaluating the overall conservatism of the ECCS Acceptance Criteria would be to conduct an extensive numerical parametric sensitivity analysis of system effects. This investigation would involve evaluating the codes' predictions of system response to systematic variations through the range of uncertainties for each of the separate LOCA model elements. One major goal of a study of this type would be to verify the assumption that prescribing individual elements of the models conservatively will results in a conservative evaluation of total system performance. Although such a program would be admittedly expensive and the present computer codes are not very satisfactory, we feel that such a parametric study would provide more insight into the relative importance of ECCS/LOCA parameters and better consequent understanding of which ones are most important for con-

\footnotetext{
${ }^{12}$ There is, however, a legitimate maturation process customarily associated with all large system level codes-the ECCS codes are no exception to this process. Comparison of recent results of Aerojet Nuclear Corporation calculations with related Semiscale experiments have shown greatly improved correlations in comparison with the earlier "Standard Problems。" Though these experiments were not conducted with the same rigorous separation of experimental team members and numerical code operators that are customary with the "Standard Problems," the coincidence of results seems to indicate that actual code improvements have been made.

Though this is very encouraging, it should be observed that maturing the codes against results obtained with the Semiscale apparatus (about $1 \mathrm{MWt}$ vs $3300 \mathrm{MWt}$ for a large power reactor) may not assure that the codes will work equally adequately when used to analyze a LOCA in a full-scale PWR. If, however, the current code versions do equally well when applied to LOFT (a 55 MWt experimental PWR) then there will be grounds for significant encouragement with respect to their adequacy. If they require additional maturation when applied to the LOFT experiments (which seems probable), then our confidence in the adequacy of the ECCS/LOCA analysis methods may not be substantially greater than it is now.
} 
servative performance estimates. Thus we feel that this type of project would be worth undertaking.

\section{d. Qualitative vs. quantitative judgments of ECCS adequacy}

The above discussion should not be taken as implying that we are terribly pessimistic about the possibility of developing adequate models and combining them into equally adequate systems analysis codes. Moreover, the inability to quantify the adequacy of ECCS performance does not necessarily imply that a safety margin does not exist. Most nuclear reactor experts are convinced that a strong qualitative bas is exists for judgments that the safety margin for the system is adequate (Cottrell, 1974). In fact, many (if not most) of the scientists and engineers involved with reactor design feel

\section{Alternate or modified ECCS concepts}

It seems apparent that alternative solutions to the ECCS problem need to be investigated. The problem would be resolved if a mechanism could be found for overwhelming the uncertainties in the current ECCS design and modeling methods. One example of such a method might be to require (through revision of the ECCS Acceptance Criteria) reactor reflooding rates with values nearer those which were originally planned for the ECCS, 6 inches/second or greater, as opposed to the marginally effective 1 to 2 inches/second estimated for current designs.

Increasing the reflooding rate to this magnitude would evidently require redesign of the ECCS in order to relieve the PWR steam binding problem. We have made no serious attempt to invent alternative ECCS designsalthough such alternatives exist and some are already under investigation. The AEC is currently funding programs to investigate alternative ECCS methods which may produce fruitful results. Moreover, LOFT has been designed to test a number of alternative ECCS concepts for PWRs which appear promising. Results of the LOFT investigations should permit at least qualitative evaluation of some alternative ECCS delivery mechanisms.

We thoroughly support the AEC's recommendation concerning PWRs that: "the calculated reflood rate should have a substantial margin over the rate that is just sufficient to turn the (LOCA) temperature excursion around in a short time... Steam binding would severely limit the rate of reflooding the core, reducing it from an intended 6 to 11 inches per second to from 1.0 to 2.5 inches per second, depending upon the reactor design... . Thus the Commission urges the pressurized-water reactor manufacturers to seek out design changes that would overcome steam binding” (AEC Commissioners, 1974, p. 1092).

Boiling-water reactors (BWRs) do not appear to be subject to the problem of steam binding, "because their system design provides a more direct path for steam to escape" (AEC Commissioners, 1974, p. 1092). Nevertheless, the same requirement exists for rapid reflooding in the BWR if high confidence in system performance is to be assured. Reflooding rates for BWRs are currently estimated to be quite high, "typically 3.7 inches per second" (AEC Commissioners, 1974, p. 1125). However, the physical size of the BWR pressure vessel introduces a rather substantial delay between the time of the accident and the time that water reaches the bottom of the core and reflooding begins (of the order of 3 minutes for the BWR as opposed to approximately one minute for a PWR). An increase in BWR flooding rates to values of 6 inches/second or more would also help to reduce the significance of uncertainties in their ECCS design.

\section{Summary}

In summary, review of the elements of Table IX shows a considerable effort on the part of the AEC to develop conservative Acceptance Criteria for the ECCS. The listed phenomena, together with the associated criteria, show the magnitude of the effort to which the AEC has gone to prescribe the individual separate effects of the LOCA in a conservative manner. Though several of these separate effects could and should be more fully understood, the really difficult question to answer is whether the individually prescribed ECCS Acceptance Criteria for the LOCA separate effects can be integrated into a meaningful system analysis with a demonstrable overall margin of conservatism. Despite qualitative indications of general conservatism with in the ECCS Acceptance Criteria, we feel that the experimental data are not adequate to demonstrate convincingly that the integrated ECC systems effects are conservatively prescribed, even if all of the individual pieces were demonstrated to be independently conservative (which they have not been). Therefore, any meaningful quantitative evaluation of system effectiveness, or the ECCS safety margin, must depend upon the adequacy of the system analysis codes. At this time, none of us has been convinced that the current generation of codes is adequate to this purpose.

We recognize that our investigation of the conservatism of the ECCS design was brief and far from exhaustive. Nevertheless, the evidence supporting code adequacy which we examined was not convincing. Since no definitive large-scale systems tests have been conducted or are currently planned, it is essential to attain confidence in the calculational methods used for design and analysis. We feel strongly that if this goal is to be achieved, without significant changes in the present complex ECCS design, it will only be through a much stronger code development program combined with a much improved experimental data base, especially in system tests. This must be coupled with a strong program in code assessment and evaluation.

A mechanism for eliminating uncertainties in ECCS performance seems highly desirable. Such a mechanism should ideally simplify both the ECCS design and the modeling requirements for evaluating it. One such mechanism appears to be increasing reflooding rates through alternate ECCS designs. No doubt other methods of achieving the goal are also possible. We strongly recommend revision of ECCS designs in order to achieve greater simplicity - with consequent simplification of modeling requirements. The goal of such improvements would be a demonstrably conservative ECCS 
concept which was empirically verifiable. We strongly support the AEC efforts to investigate alternate ECCS concepts. Moreover, the AEC should continuously monitor the development of alternate methods and move to require their utilization as soon as it is apparent that an improved method is available. Though major changes in ECCS design appear desirable, incorporation of small improvements into the current designs, if they can be shown to be of value, should not be precluded.

\section{Containment behavior in a LOCA}

This section contains a discussion of the functioning and behavior of the containment building and systems during the course of a loss-of-coolant accident (LOCA). The first portion discusses containment behavior in a LOCA with effective emergency core cooling so that there would be no core melt. The second portion discusses what would happen in a LOCA in which the emergency core cooling did not prevent core meltdown. Finally, we offer some suggestions regarding improved containment.

\section{Containment performance with effective emergency core cooling}

Most PWR containment buildings are sized and designed to withstand the maximum temperature and pressure expected from the steam generated in a LOCA by vaporization of the expelled primary coolant. BWR containment designs depend on quenching the steam as it is formed. In a LOCA the steam would have to force its way through water into a secondary containment volume.

The primary coolant water would contain some radioactivity which would be vented into the containment volume. The containment has systems (e.g., sprays, filters, etc.) intended to remove much of this radioactivity from its atmosphere and to remove decay heat from the containment atmosphere in order to prevent overpressurization. Post-accident heat removal would be performed by systems that transfer heat from heated water inside the containment to cold water outside the containment. The water that flows through the primary side of the heat exchangers would be taken from the reactor building sump in the PWR and from the pressure-suppression pool in the BWR. Failure to perform the postaccident heat removal could lead to steam overpressure failure of the containment. Other systems would remove hydrogen which could be produced by radiolytic decomposition of water and by metal-water reactions.

The radioactivity released through the break in the primary system during a LOCA with effective emergency core cooling would mostly be retained inside the containment building if the containment atmosphere cooling and isolation systems work as designed. Although these systems appear to be straightforward in their engineering design, we feel that a review of this capability is appropriate.

\section{Containment performance in a LOCA without effective emergency core cooling}

In Appendix I. B we discuss the sequence of events in an accident without effective emergency core cooling and trace the development through seven periods. We are concerned here with the seventh period, in which an uncooled core would heat up to its melting point and melt through the bottom of the pressure vessel in a period of the order of an hour. The core material, together with the molten vessel and other material, would weigh on the order of 100-200 tons. A steam explosion might subsequently occur when the molten mass falls into the water remaining in the cavity below the pressure vessel. In any case, the water would be quickly displaced and the concrete of the containment floor beneath the molten core would spall and disintegrate, releasing $\mathrm{CO}_{2}$ and water vapor as it heated up. Since the mixture of steel and molten fuel is denser than the concrete ingredients, the concrete residue would either mix with the molten mass or float to the top. It is estimated in Draft WASH1400 (Appendix VIII, pp. A-35-A-39) that containment melt-through would occur in a period on the order of a day.

The more volatile fission products would be released from the molten mass and some would recondense on cooler containment surfaces. This would result in a significant heat source in the containment atmosphere and on its surfaces.

The containment would be breached within a day, probably by one of the following five mechanisms:

1. If the containment atmosphere cooling system and the isolation valves work in a PWR containment (an 85\% probability, according to Draft WASH-1400), the containment would not be breached until the core melted through the floor. The radioactive gases released in this manner would then only be able to reach the atmosphere after seeping through the ground under the containment where they might be substantially attenuated by the filtering action of the soil. An attenuation factor ranging from 100 to 1000 is assumed in Draft WASH 1400 to result for all fission products other than the noble gases. We believe that some filtering would occur in the soil but are concerned that "blowouts" or channeling might bypass the soil filter to some extent. The driving force for such channeling could be provided by the gas pressure in the containment or by pressure pulses from steam explosions when the molten core contacts ground water. Such channeling mechanisms are not discussed adequately in Draft WASH-1400.

The timing and manner of containment failure, particularly the height of the release from the containment to the atmosphere, would have a large influence on radiation doses near such a major accident but have little influence on the long-delayed fatalities and health effects which are the main subject of this chapter.

2 . If the containment atmosphere cooling system were to fail, the radioactive decay heat and the heat released by chemical metal-water reactions could raise the steam pressure in the containment to the containment failure threshold in a period of hours.

3. Metal-water reactions would generate hydrogen, and concrete decomposed by the heat of the core would release $\mathrm{CO}_{2}$. These noncondensible gases might by themselves raise the gas pressure in a small BWR primary containment to the containment failure threshold. 
Hydrogen could form a combustible mixture with any oxygen already in the containment atmosphere.

4. The containment isolation valves could fail to close, leaving a large leakage path to the outside.

5. A steam explosion, resulting from the autocatalytic mixing of a large portion of the molten core with water in the bottom of the reactor pressure vessel or in the pit below, might rupture the containment and disperse a significant fraction of the core into the atmosphere.

If there were a steam explosion in the pressure vessel, the danger to the containment integrity would probably be greatest if the steam explosion were severe enough to blow off the top head of the pressure vessel. The head could have sufficient kinetic energy to reach the dome of the containment building and cause failure. The containment is protected from projectiles in other directions by the missile barriers normally meant to shield the pressure vessel. The containment dome may be somewhat shielded by the overhead crane.

A steam explosion of sufficient magnitude to rupture the containment by its pressure wave would require interaction of a large fraction of the core and would be unlikely to occur in most cases. The mechanisms of steam explosions are not well understood and the likelihood of steam explosions involving even a small part of the core is unknown.

\section{Improvements in containment}

We feel it is appropriate to consider the possibility of designing increased effectiveness into the containment in case of core meltdown. Various proposals which we believe deserve further exploration are discussed briefly below and more extensively in Appendix II and Sec. VI.

\section{a. Controlled failure}

According to Draft WASH-1400, containment failure before melt-through would often occur through overpressure. It would thus be useful to have the containment designed so as to fail in a controlled way in case of overpressure, i.e., with a reliable passive pressure relief valve system and filter bed so that the containment atmosphere would be scrubbed and filtered as it is released.

\section{b. Underground siting}

Underground siting offers enough potential safety advantages to deserve more serious exploration than it has received in this country to date. Four European reactors-three research and one commercial-have been sited underground. The potential advantages include filtering by the over-burden of any radionuclides which escape the reactor cavity, less shaking by earthquakes, added protection against the accidental or delibe rate impact of aircraft and missiles, and the gravity head of surface reservoirs of emergency cooling water. The additional filtration of any release seems to be a most important aspect of underground siting as it affects the potential consequences of a large LOCA.

\section{c. Core catchers}

There has been periodic speculation during the past decade about the feasibility of a core catcher, a device which would hold the molten core and prevent it from melting through the base of the containment. The requirement of such a device was seriously considered when the current generation of 1000 MWe reactors was being introduced and also for the Fast Flux Test Facility and Clinch River Breeder Reactors. Serious engineering studies of the more promising ideas which have been proposed would appear timely.

\section{d. Pressure suppression and isolation valves}

Draft WASH-1400 indicated that following a core melt there would be a significant chance of containment failure through overpressure because of failure of the containment atmosphere cooling system and, to a lesser extent, loss of containment integrity due to penetrations and failure of isolation valves. It appears worthwhile to improve the reliability of pressure suppression, heat removal, and isolation systems.

\section{e. Effectiveness of containment spray and filtering systems}

Draft WASH-1400 concludes that in most meltdown accidents, PWR containment spray and filtering systems and BWR pressure-suppression pools would scrub a large fraction of the suspended radioactivity out of their respective containment atmospheres. These appear to be important engineered safeguards and we believe that their reliability and effectiveness should be verified.

\section{E. The release of radionuclides and consequences of a core melt}

This section discusses the radionuclides that would be released if there were a core melt, how they might be transported in the atmosphere, and the consequences of the radiation doses and of land and property contamination. (Appendix II of this report discusses these effects in more detail and gives references to the literature.)

\section{Radionuclides released from a molten core}

The radioactive inventory of an operating LWR is several billion curies of radioactive isotopes with halflives of the order of an hour or longer. (See Table XXXIV)。The extent to which these isotopes would be released from the molten core and the nature of the hazard they represent to man depends on their chemical properties. The principal radionuclides may conveniently be grouped chemically as follows:

\section{a. Noble gases $(\mathrm{Xe}, \mathrm{Kr}$ )}

Since these are gases at the temperatures of interest and are not strongly bound chemically, they would escape from the melt. The hazard from radioactive noble gases is primarily through external gamma radiation, since they are not absorbed in large amounts into the human body. 


\section{b. lodine}

The boiling temperature of iodine is about $360^{\circ} \mathrm{F}$ and in laboratory experiments it is found not to be bound to the melt effectively. The primary hazard from radioactive iodine is due to the fact that it is readily absorbed by the human body after inhalation or ingestion and is concentrated in the thyroid. The dose of radiation which would be received by this small organ after a meltdown accident in which the containment fails is, the refore, likely to be considerably larger than the dose received by any other organ. A large fraction of the iodine would be expected to be associated with the aerosol formed by the condensed metal oxides vaporized under the high temperature, and the other vapors which would be boiled off from the melt. It would take many hours for this aerosol to plate out on the containment interior by natural processes.

\section{c. Cesium and tellurium}

The boiling temperatures of these elements are about $1250^{\circ}$ and $1800^{\circ} \mathrm{F}$, respectively and, on the basis of lab-oratory experiments, it appears that they would not be retained appreciably in the melt. The principal immediate hazard from these and other isotopes to be discussed below would be through internal irradiation of the body following inhalation. Cesium-137 is also of major concern as a land contaminant due to its long half-life (30 years) and the penetrating gammas associated with its decay chain.

\section{d. Volatile oxides (Mo, Tc, Rh, Ru)}

The boiling points of these elements are well above the melting temperatures of $\mathrm{UO}_{2}$, but molybdenum and technetium have stable oxides which are volatile at much lower temperatures. In the presence of sufficient free oxygen, therefore, one would expect them to oxidize and escape the melt. Suitable conditions would exist if a steam explosion were to disperse some of the melt into the atmosphere. Whether there would be sufficient free oxygen in the melt to oxidize these elements fully under ordinary meltdown conditions is unclear. Ruthenium and rhenium oxides are less stable and their release mechanism would be more complex. The release of ruthenium from molten fuel based on laboratory experiments varies from tenths of percents to nearly complete release in a pattern which has not been adequately explained.

\section{e. Alkaline earths ( $\mathrm{Ba}, \mathrm{Sr})$}

These elements are relatively volatile but, in the presence of free oxygen, $\mathrm{CO}_{2}$, or $\mathrm{H}_{2} \mathrm{O}$, they form less volatile oxides ( $\mathrm{Ba}$ is semivolatile at $\mathrm{UO}_{2}$ melting temperatures while $\mathrm{Sr}$ is nonvolatile). The releases in laboratory experiments vary from tenths of percents for $\mathrm{Sr}$ and percents for $\mathrm{Ba}$ in oxidizing conditions to the order of ten percent under reducing conditions. ${ }^{90} \mathrm{Sr}$ is a major concern as a land contaminant due to its long half-life (28 years) and its efficient delivery to man via the ground-grass-cow-milk route.

\section{f. Nonvolatile oxides $(\mathrm{Zr}, \mathrm{Nb}, \mathrm{La}, \mathrm{Ce}, \mathrm{Pr}, \mathrm{Nd}, \mathrm{Pm}$, Sn, $Y, P u)$}

These elements all react with $\mathrm{H}_{2} \mathrm{O}$ and $\mathrm{CO}_{2}$ to form stable oxides. Both $\mathrm{H}_{2} \mathrm{O}$ and $\mathrm{CO}_{2}$ would be produced in abundance by the decomposition of the concrete in the reactor containment floor under the molten core. The oxides would mix with the $\mathrm{UO}_{2}$ and would be expected to be released roughly in proportion to the $\mathrm{UO}_{2}$ which is vaporized. Some of the transuranic isotopes with their long half-lives $\left({ }^{238} \mathrm{Pu}, 86\right.$ years; ${ }^{239} \mathrm{Pu}, 24000$ years; ${ }^{240} \mathrm{Pu}, 6500$ years; ${ }^{242} \mathrm{Pu}, 279000$ years; ${ }^{241} \mathrm{Am}, 460$ years; ${ }^{243} \mathrm{Am}, 8000$ years; ${ }^{244} \mathrm{Cu}, 18$ years), their alpha decay modes, and their multi-kilogram inventories, are of particular concern as land contaminants. In the case of plutonium, at least, the oxides are quite insoluble and would remain in the soil. If close to the surface, they might be available for resuspension and inhalation. Following inhalation, they could be retained in the lungs for long periods with the energetic alpha particles depositing all of their energy in the lung tissue.

In the absence of a good model of core boil-off, we estimate that following a core meltdown, as much as a few percent of the core (and therefore the nonvolatile oxides) could be boiled off and suspended as an aerosol in the containment volume (about $2 \times 10^{6}$ cubic feet) of a 1000 MWe PWR.

Our conclusions on release fractions are largely in agreement with those of Draft WASH-1400. We note that the data reviewed there regarding release of elements from simulated melts do not seem to preclude release fractions for some volatile and nonvolatile oxides that could be larger or smaller by approximately an order of magnitude than the values adopted in Draft WASH -1400 .

\section{Transport of released radioactivity}

\section{a. Atmospheric dispersion}

The initial behavior of any radioactive gases released into the atmosphere would depend sensitively on the height and nature of the release from the containment and on the atmospheric conditions. The heat of the released gases might result in the plume rising initially by a few hundred meters - or the turbulent wake of the containment building might prevent the plume from rising clear of the ground. Depending on the stability of the atmosphere, the plume could have a horizontal

\footnotetext{
${ }^{13}$ Compared to atmospheric motions, ground water movement is very slow. For the longer-lived fission products such as ${ }^{90} \mathrm{Sr}$, however, it can be significant. Thus, in a study of the ground water under the Chemical Processing Plant at the National Reactor Testing Station in Idaho (now the Idaho National Engineering Laboratory), it was found that ${ }^{90} \mathrm{Sr}$ injected into an aquifer through a deep well had migrated approximately 1 kilometer in 8 years. This distance is comparable to the distance of many commercial nuclear power plants from the large bodies of water which supply their cooling water. A large fraction of the radionuclides in the core might be expected to be trapped in the cooled melt when the core comes to rest under the containment, but the fraction which is boiled off and trapped in the soil would be readily accessible to the ground water.
} 
dispersion ranging from about 0.1 to 0.7 radians. Also, depending on atmospheric conditions and wind speed, the vertical dispersion could reach $100 \mathrm{~m}$ "standard deviation" as close as $0.5 \mathrm{~km}$ downwind or, under other circumstances, perhaps barely that much at distances as great as $100 \mathrm{~km}$ downwind.

The uncertainties in the modelling make it difficult to predict the consequences of an uncontained meltdown accident close in to the reactor-particularly in view of the highly nonlinear effects of large radiation doses in causing acute illness or immediate fatalities. For example, a $200 \mathrm{rem}$ whole-body dose might kill less than $5 \%$ of the exposed population within 60 days, while 350 rem might kill $50 \%$. In addition, there would be large variations in close-in consequences for a given release as a function of variations in weather and population densities.

At greater distances, however, low-dose effects would replace acute effects as the focus of concern. Here the complexities of meteorological phenomena andrelease conditions become less important. The primary reason for this relative simplicity is due to the standard assumption that for subacute doses the effects of concern (cancer and genetic defects) are linearly dependent on the population radiation dose measured in man-rem. Thus, for example, it is assumed that an equal number of cancers will result from 100000 people each receiving a whole-body dose of $10 \mathrm{rem}$ as from 50000 people each of whom receives 20 rem. We categorize effects assumed to have this simplifying property as "man-rem" consequences. This important assumption was used in Draft WASH-1400 to calculate delayed fatalities and genetic defects; it is discussed in some detail in Appendix II-C.2.

\section{b. The wedge model}

Man-rem consequences are calculated in Draft WASH-1400 in a complex computer model. In order to calculate the man-rem consequences in a transparent way, we have used the "wedge model," a simplified model of atmospheric dispersion, to examine a number of characteristic situations. The results obtained from this model compare very well with those in Draft WASH -1400 where comparison is possible.

This approximation takes advantage of the fact that the population dose is dominated by the large number of small individual doses hundreds of miles downwind from the reactor accident. At such distances, the plume will usually have diffused uniformly through the lower "mixing layer" of the atmosphere, which usually has a thickness of the order of $1000 \mathrm{~m}$. The population dose is essentially independent of the horizontal density distribution of the plume. We can, therefore, approximate the transverse density distribution of the plume as uniform within a wedge with an opening angle averaging approximately 0.25 radians and as zero outside this opening angle-hence the name "wedge model."

The wedge model is described in detail in Appendix II where it is used to calculate the various population doses associated with the important radionuclides released from the core.

In the next section we illustrate the use of the model by calculating the long-term whole-body external gamma dose from ${ }^{137} \mathrm{Cs}$. This single radionuclide is responsible for more than $80 \%$ of the whole-body population dose which would be associated with the Draft WASH-1400 "Reference Accident." This accident, also designated as PWR2, represents a meltdown at a 1000 MWe PWR with essentially complete containment failure. It is also the hypothetical accident whose consequences are presented in most detail in Draft WASH-1400. As will be shown below, we have concluded that this total population dose, most of which was neglected in Draft WASH-1400, would be of major significance in such an accident.

\section{F. Synopsis of the reference accident consequences due to ${ }^{137}$ Cs alone}

The large number of radionuclides considered in our analysis, and the many special effects of radiation on various organs, conceal the fact that a single isotope, ${ }^{137} \mathrm{Cs}$, provides more than $80 \%$ of the total whole-body man-rem dose to the population in the plume (Tables XIII; XLI). Much of this dose was not included in the dose calculated in Draft WASH-1400. We shall calculate here the effects due to ${ }^{137} \mathrm{Cs}$ alone to illustrate the more complete calculation we present in Appendix II and to draw attention to the important assumptions.

The amount of ${ }^{137} \mathrm{Cs}$ in the reactor core at the time of the assumed release is $A_{0}=5.8 \times 10^{6}$ curies, and its half-life $\tau$ is 30 years (Table XXXIV). In the Draft WASH-1400 reference accident, a fraction $F_{R}=0.50$ of the cesium present in the reactor core is assumed to be released to the atmosphere from the containment (Table XI). We assume the released radioactivity to be transported at a speed $u=3.5 \mathrm{~m} / \mathrm{sec}$ thoroughly mixed in a wedge-shaped plume of air depth $H=1100 \mathrm{~m}$ and of angular width $\theta=0.25$ radian. ${ }^{137} \mathrm{Cs}$ is assumed to deposit on any surface exposed to the plume at a rate determined by its average concentration $c(r, t)$ in the plume, together with a "deposition velocity" $v_{d}=0.2$ $\mathrm{cm} / \mathrm{sec}$ (Table XII), where $r$ is the distance downwind from the source. Thus we assume the radioactivity to be swept along the wedge at constant speed $u$ in the form of a thin "front" of thickness $\Delta r$. The deposition of radioactivity on the ground at a distance $r$ from the source, the dose given to individuals by passage of the cloud, and the inhalation dose all depend on a time-integrated concentration of radioactivity

$$
\chi(r)=\int_{t=0}^{\infty} c(r, t) d t
$$

where $\chi(r)$ is measured in curie-sec per $\mathrm{m}^{3}$. (The more general derivation of the wedge model, including depletion of the plume radioactivity by decay and deposition, is given in Appendix II.)

The initial ground contamination in curie $/ \mathrm{m}^{2}$ is

$$
\begin{aligned}
G(r) & =\left(A_{0} F_{R} / H \theta r \Delta r\right)\left(v_{d} \Delta r / u\right) \\
& =\left(A_{0} F_{R} v_{d} / H \theta r u\right) .
\end{aligned}
$$

With our assumptions, this is $G(r)=6000 / r$ microcurie/ $\mathrm{m}^{2}(r$ in $\mathrm{km})$. The initial ground contamination $G(r)$ produces a dose in air above smooth ground which is ini- 
tially $D_{0}=C_{D G} G$, where $C_{D G}$ is $0.12 \mathrm{rad} /$ year per $\mathrm{microcurie} / \mathrm{m}^{2}$ (Eq. AII-23). Because of natural attrition and movement of the ${ }^{137} \mathrm{Cs}$ in the soil, the dose rate drops off more rapidly than the natural decay and integrates instead to $D_{\mathrm{int}}=I_{D G} G$, where $I_{D G}$ is $1.55 \mathrm{rad}$ per microcurie $/ \mathrm{m}^{2}$. To obtain the integrated wholebody dose $E_{\text {wb }}$ to persons in dwellings, we assume a shielding factor $F_{s}$ of 0.33 (see Appendix II).

Thus the dose to the average person at radius $r \mathrm{~km}$ is

$$
\begin{aligned}
E_{\mathrm{wb}}(r) & =F_{s} D_{\mathrm{int}}=F_{s} I_{D G} G=(0.33)(1.55) G(r) \\
& =3120 / r \text { rem } \quad(r \text { in } \mathrm{km}) .
\end{aligned}
$$

As will be discussed below, this whole-body exposure gives rise to increased probability of death from cancer arising typically 10 to 30 years after exposure

$$
C(r)=C_{C E} E_{\mathrm{wb}}(r),
$$

where $C_{C E}$ is taken as $1.3 \times 10^{-4}$ per rem (Appendix II, Section IV). Thus,

$$
C(r)=0.4 / r, r \text { in } \mathrm{km} \text {. }
$$

It is assumed in Draft WASH-1400 that people will be evacuated within one day from the regions near the hypothesized "Reference Accident" where the contamination due to the plume exceeds a threshold $G_{\mathrm{th}}=100$ microcurie $/ \mathrm{m}^{2}$. For our assumptions this would occur at $r_{i}=60 \mathrm{~km}$, according to Eq. V-2. If no effective decontamination is achieved beyond a radius $r_{i}$, the average additional cancer death probability downwind within the narrow plume would range from an estimated $0.7 \%$ at $60 \mathrm{~km}$ to some $0.05 \%$ at $800 \mathrm{~km}$ (the cutoff we assume for our calculation). This individual risk is independent of population density but is inversely proportional to the assumed plume angular width $\theta$ (here taken to be 0.25 radian). On the other hand, the mean number of such latent cancer deaths following the reference accident is independent of $\theta$, but depends on the assumed population density which is here taken to be $N_{\text {pop }}=300$ per square mile, or $116 / \mathrm{km}^{2}$. The mean number is then

$$
\begin{aligned}
N_{C} & =\int_{r=r_{i}}^{r=R} C(r) r \theta N_{\mathrm{pop}} d r \\
& =\mathbf{1 2}\left(R-r_{i}\right),
\end{aligned}
$$

or formally

$$
N_{C}=\left(R-r_{i}\right) F_{s} N_{\text {pop }} C_{C E} I_{D G}\left(A_{0} F_{R} v_{d} / H u\right) \text {. }
$$

The corresponding man-rem dose to the exposed population is thus

$$
E_{\text {man-rem }}=\left(R-r_{i}\right) N_{\text {pop }} F_{s} I_{D G}\left(A_{0} F_{R} v_{d} / H u\right), \quad(\mathrm{Eq} \cdot \mathrm{V}-5)
$$

and under our assumption gives a total population exposure of 70 million man-rem.

This result is independent of the assumed plume width so long as only a small fraction of the population out to $R=800 \mathrm{~km}$ is evacuated. This population exposure under the assumed conditions results in an additional 9000 long-delayed cancer deaths from ${ }^{137} \mathrm{Cs}$ ground contamination.

In this brief analysis, we shall not reproduce the corresponding simple calculations of genetic defects and other damages due to whole-body irradiation dose, which are presented in Table XIV. We wish, however, to call attention to the sensitivity of our result to various assumptions and coefficients. From Eq. V-4 we see that the number of latent cancer deaths is directly proportional to:

$A_{0}$ the inventory of ${ }^{137} \mathrm{Cs}$ in
the reactor core

$F_{R}$ the fraction released in the reference accident

$5.8 \times 10^{6}$ curies

the deposition velocity of $\mathrm{Cs}$

from the plume
$I_{D G}$ the dose (rad) in air per microcurie $/ \mathrm{m}^{2}$ of ${ }^{137} \mathrm{C}$ on the ground

$0.2 \mathrm{~cm} / \mathrm{sec}$

$F_{s}$ the shielding factor between $\mathrm{rad}$ in air and rem

$N_{\text {pop }}$ the population density in persons $/ \mathrm{km}^{2}$

$R$ the distance to which the integrals are taken

$800 \mathrm{~km}$

$C_{C E}$ the number of latent cancers per man-rem and inversely proportional to:

$H$ the thickness of the atmospheric mixing layer

$1.3 \times 10^{-4}$

the mean wind speed

$1100 \mathrm{~m}$

$3.5 \mathrm{~m} / \mathrm{sec}$

The uncertainties in these assumptions are discussed in later sections of this chapter and in Appendix II.

The average cancer death risk to the individual within the narrow plume at $400 \mathrm{~km}$ as a consequence of this assumed reference accident, which has a probability of the order of $6 \times 10^{-6}$ per reactor year according to Draft WASH -1400 , would be about $0.1 \%$. The fatalities would probably not begin for 5 to 10 years and would be spread over the natural lifetimes of the inhabitants. This $0.1 \%$ additional risk of death from cancer to an exposed individual following such a low-probability event should be compared with the approximately $20 \%$ of the entire population who will die from cancer having nothing to do with nuclear reactors.

\section{G. Other radiation doses from radionuclides released to the atmosphere}

In the preceding section we considered only the ${ }^{137} \mathrm{Cs}$ dose effects. We indicate here the procedure to be used in computing other dose effects. To obtain whole-body external radiation dose from the passing cloud one needs to multiply $\chi$ of Eq. AII-16 (which is the extension of Eq. $\mathrm{V}-1$ to include depletion and decay) by a dose-exposure coefficient such as those given in Table XXXVII and by a shielding factor if one is indoors.

To obtain the doses to the whole body and various organs resulting from inhalation of the radionuclides in the cloud, one multiplies $\chi$ by a breathing rate $\left(\approx 2 \times 10^{-4}\right.$ $\mathrm{m}^{3} / \mathrm{sec}$ for an adult) and then by an appropriate dose-exposure coefficient such as those given in Tables XXXV and XXXVI.

Finally, to obtain the external radiation dose rate from ground contamination, one first multiplies $\chi$ by the appropriate deposition velocity to get the ground contamination (measured in curies $/ \mathrm{m}^{2}$ ), and then multiplies by the 
appropriate dose-exposure coefficient such as those given in Table XXXVII.

\section{H. Land contamination thresholds}

The short-term population radiation dose is dominated by radionuclides inhaled from the radioactive plume. However, in the long term, a much larger population dose may result from land and water contamination. This long-term dose may be delivered via many routes:

\section{Direct gamma irradiation from contaminated ground}

The principal concern is the 30-year half-life isotope ${ }^{137} \mathrm{Cs}$ which ordinarily would become fixed in the soil by ion exchange. For any uniform population density, we find that about $75 \%$ of the long-term ground-dose to humans would come from ${ }^{137} \mathrm{Cs}$, and some 90 percent from the combined contributions of ${ }^{137} \mathrm{Cs}$ and two-year half-life ${ }^{134} \mathrm{Cs}$. Unfortunately, it is difficult to calculate a reliable dose associated with any particular level of land contamination. For example, in calculating the dose rate in dwellings compared to that in an open field, the walls of the dwellings would offer some shielding against the gamma rays from ground contamination, but there might be some "antishielding" effects as well from buildings, since the contamination would be in the form of a fine aerosol which could cling to walls and roofs. With regard to decontamination, although $\mathrm{Cs}$ is chemically active and its salts generally soluble, it is by no means certain that it can be cleaned from walls by any means short of sandblasting. Roofs might have to be replaced. Faced with such complexities, we will follow the procedure used by the United Nations Scientific Committee on the Effects of Atomic Radiation (UNSCEAR, 1972 ) in calculating time dependence of doses from ${ }^{137} \mathrm{Cs}$ in fallout (Eq. AII-23). The U.N. Committee uses a factor of 0.40 to convert rads-in-air to whole-body dose, and a factor of 0.32 to convert rads-in-air to dose to the gonads. Draft WASH-1400 uses a factor 0.33 for both conversion factors, and for simplicity we also use this same factor in our calculations.

\section{Contamination of foodstuffs by radionuclides}

Of special concern, in addition to ${ }^{137} \mathrm{Cs}$, are the 8 -day half-life isotope ${ }^{131} \mathrm{I}$ and the 28-year half-life isotope ${ }^{90} \mathrm{Sr}$, both of which are concentrated particularly efficiently via the grass-cow-milk route. In the absence of any diversion of contaminated milk, the average thyroid dose from ${ }^{131}$ I via this route would range from a factor of 7 higher than that due to inhalation, in the case of adults, to a factor 100 higher in the case of infants (EPA, 1973, p. 127).

\section{Contamination of surface water}

By contamination of the surface water by fallout or of ground water by radioisotopes which might accompany the molten core if it melts through the base of the reactor containment, ${ }^{90} \mathrm{Sr}$ is again of special concern.

\section{Contamination of soil}

By contamination of the soil with small insoluble radioactive particles which might be resuspended by winds or human activities and subsequently inhaled, plutonium is of special concern.

All these sources of irradiation are in principle avoidable by evacuation of contaminated areas, by taking such land out of agriculture, and by condemning contaminated foodstuffs and water supplies. In practice, there would be a level of contamination below which the costs of these measures would outweigh the corresponding reduction of risk. The suggested contamination thresholds in Table $X$ from Draft WASH-1400 should be the subject of much more serious and open discussion before final values are set.

To obtain an idea of what is involved in a cost benefit calculation associated with the setting of a contamination threshold in the absence of effective decontamination techniques, consider the case of ${ }^{137} \mathrm{Cs}$. According to the calculations in Sec.V.F, the individual cancer death risk associated with the reference accident goes from $0.7 \%$ at the distance of $60 \mathrm{~km}$, corresponding to the Draft WASH-1400 evacuation threshold of $100 \mu$ curies $/ \mathrm{m}^{2}$, to $0.10 \%$ at 400 $\mathrm{km}$ where the contamination has dropped to $15 \mu$ curies / $\mathrm{m}^{2}$. Considering the standard calculation of man-rem effects to population out to $800 \mathrm{~km}, \mathrm{Eq} . \mathrm{V}-5$ shows that the total man-rem dose can be approximately halved by early evacuation out to $400 \mathrm{~km}$. Thus half the latent cancers and half the genetic defects might be eliminated at the cost of long-term (5 to 10 years or more) evacuation of an area of some 7500 square miles. With a population density of 300 people per square mile, this would mean the evacuation of some 2 million people.

The area which would have to be evacuated for a long time in the case of a major release of radioactivity from a reactor accident would be much reduced if effective decontamination techniques could be implemented. The dose rate from open ground could be reduced significantly, for example, by deep plowing. The soil would then act as a shield against the gamma rays from a significant fraction of the radionuclides. The mean free path of gamma rays from ${ }^{137} \mathrm{Cs}$ in soil is about $7 \mathrm{~cm}$. How ever, the costs and effectiveness of decontamination techniques in populated areas are not well understood.

\section{Comparison with analysis in Draft WASH-1400 of reference accident (PWR2) consequences}

In this section we compare our wedge model analysis of consequences of the reference accident (PWR2) of

TABLE X. Land contamination thresholds (Draft WASH-1400, Appendix VI, p. 66).

\begin{tabular}{lccc}
\hline \hline Isotope & $\begin{array}{c}\text { Half-life } \\
\text { (years) }\end{array}$ & $\begin{array}{c}\text { Use for human } \\
\text { occupation } \\
\left.\text { (microcurie per } \mathrm{m}^{2}\right)\end{array}$ & $\begin{array}{c}\text { Use for } \\
\text { agriculture } \\
\text { (microcurie per } \mathrm{m}^{2} \text { ) }\end{array}$ \\
\hline $\mathrm{Sr}^{89}$ & 0.14 & 50 & 10 \\
$\mathrm{Sr}^{90}$ & 27.7 & 20 & 2 \\
$\mathrm{I}^{131}$ & 0.022 & 1000 & 5 \\
$\mathrm{Cs}^{134}$ & 2 & 20 & 10 \\
$\mathrm{Cs}^{136}$ & 0.035 & 200 & 100 \\
$\mathrm{Cs}^{137}$ & 30 & 100 & 20 \\
$\mathrm{Ce}^{144}$ & 0.78 & 500 & 100 \\
$\mathrm{Pu}^{238}$ & 86.4 & 0.5 & 0.2 \\
$\mathrm{Pu}^{239}$ & 24390 & 0.2 & 0.1 \\
\hline \hline
\end{tabular}


Draft WASH -1400 with the analysis of that accident in Draft WASH-1400. This accident assumes that there is a core meltdown for a 1000 MWe PWR with a subsequent failure of containment. This accident, which can be used to set a scale for reactor accident consequences, is the accident for which the most complete information is given in Draft WASH-1400.

As discussed earlier, there are two major categories of consequences: immediate effects on relatively small areas and populations, and long-term effects on larger areas and populations. Since the prompt effects are highly sensitive to detailed assumptions as to meteorological conditions, biological thresholds, and so forth, we have made no effort to present an independent assessment of these consequences (Draft WASH-1400 estimates an average of 62 immediate deaths; a partial update of these results estimates an average of 169 immediate deaths for PWR2).

For the long-term consequences, we are able to calculate consequences from total long-term man-rem population doses in a particularly transparent way because of the linear hypothesis and the consequent averaging effect on large populations and areas.

In many areas of overlap with Draft WASH-1400, we are in agreement with the Draft, but in others there is substantial disagreement. We compare our results with those of Draft WASH-1400 as follows below: (1) computed population doses; (2) computed consequences of these doses; and (3) a review of the differences found. This comparison shows some important omissions in Draft WASH-1400, as well as differences between our analysis and that of Draft WASH-1400. We understand that these are under consideration in the revisions leading to the final WASH-1400 report (NRC, $1975 \mathrm{a}, \mathrm{b}) .{ }^{14} \mathrm{De}-$ tails of the calculations and comparisons are given in Appendix II.

\section{Calculation of population doses for the reference accident}

For purposes of comparison we have tried to use the same assumptions made in Draft WASH-1400. We have used in our calculations the release fractions from the Draft WASH -1400 as shown in Table XI. As stated above,

\footnotetext{
${ }^{14}$ In order to reduce the risk of publishing a report containing major misunderstandings, we submitted in writing to personnel of the Nuclear Regulatory Commission a set of questions covering the technical basis for the differences in our results from those of Draft WASH-1400. We received replies to these questions at a meeting in Washington, March 17, 1975, and in a communication, dated March 27, 1975. NRC is working to convert Draft WASH-1400 to a final report, and many refinements and corrections have been addressed by the group doing this work. Several of the discrepancies between our results and those of Draft WASH-1400 calculated for the same assumptions turn out to arise from a lack of consistency between the input to the computer program used in deriving the results of Draft WASH-1400 and the text of the Draft itself. In general, we continue to refer to the data presented in Draft WASH -1400 , calling attention to important changes that have been called to our attention by the indication, "(NRC, 1975a)" or “(NRC, 1975b)." Draft WASH-1400 was a Draft, and we hope that our work will help the authors in their efforts to pro-duce as accurate and authoritative a document as possible.
}

TABLE XI. Radionuclide atmospheric release fractions for the Draft WASH-1400 reference accident (Draft WASH-1400, Appendix VI, p. 9).

\begin{tabular}{lc}
\hline Principal chemical groups & $\begin{array}{c}\text { Fractional release to } \\
\text { atmosphere from core }\end{array}$ \\
\hline Noble gases & 0.9 \\
Iodines & 0.7 \\
Cesiums & 0.5 \\
Telluriums & 0.3 \\
Alkaline earths & 0.06 \\
Volatile oxides & 0.02 \\
Nonvolatile oxides & 0.004 \\
\hline
\end{tabular}

while our conclusions on these release fractions are largely in agreement with Draft WASH-1400, we note that the release fractions for some volatile and nonvolatile oxides could be larger or smaller by an order of magnitude. The meteorological parameters and population density ${ }^{15}$ which we have used are averages over the values used in Draft WASH-1400 and are given in Table XII. The comparison of our calculated population doses with those available in Draft WASH -1400 is given in Table XIII.

Our direct comparison with Draft WASH-1400 as shown in Table XIII showed some substantial discrepancies for cloud dose and one-day ground dose. More recent data (NRC, $1975 \mathrm{a}, \mathrm{b})$ bring the Draft WASH-1400 results con-

\footnotetext{
${ }^{15}$ Draft WASH-1400 (Appendix VI, p. 26) states: "To give the reader a comprehension of the numerical values involved, plots of the average (all 66 sites) cumulative population and population density are given in Figures VI-3 and VI-4. Note that beyond 30 miles the average population density is constant-and its value, approximately 300 people per square mile, is much higher than the $U$. S. average population density (excluding Alaska) of approximately 70 people per square mile. Table VI-6 gives the cumulative population at selected distances for the thirteen (siting) categories. In each category, the population from 70 to 500 miles was computed on the basis of the average population density from 0 to 70 miles, or 300 people/square mile, whichever is less."
}

Unable to determine from the above the average population density used in the Draft WASH-1400 calculation of PWR2 consequences, we requested and obtained (NRC, 1975a) this information:

For 13 synthesized site categories, population density (people $/ \mathrm{mi}^{2}$ ) beyond 70 miles was:
$\frac{\text { Minimum }}{0}$ $\frac{\text { Average }}{165}$ $\frac{\text { Maximum }}{300}$

Our initial understanding was that a population density of 300/ $\mathrm{mi}^{2}$ was used in Draft WASH-1400 calculations of man-rem consequences out to 500 miles, and we used this value in our own estimates to allow direct comparison with the results of Draft WASH-1400. Our present understanding is that Draft WASH-1400 man-rem consequences were calculated for an average density of 165 persons per square mile, and our own totals for man-rem consequences (cancers and genetic defects) should be multiplied by a factor 0.55 for direct comparison.

For any actual release, of course, as contrasted with the averaged results for the hypothetical PWR2 accident, the manrem consequences will be proportional to the existing population density. 
TABLE XII. Meteorological and other parameters used (see Appendix II for details of calculation).

\begin{tabular}{ll}
\hline \hline Wind speed $(u)$ & $3.5 \mathrm{~m} / \mathrm{sec}$ \\
Inversion height $(H)$ & $1100 \mathrm{~m}$ \\
Plume angular width $(\theta)$ & $0.25 \mathrm{radians}$ \\
Deposition velocities $\left(v_{d}\right)$ & \\
$\quad$ Iodines & $0.5 \mathrm{~cm} / \mathrm{sec}$ \\
Noble gases & $0.0 \mathrm{~cm} / \mathrm{sec}$ \\
Other isotopes & $0.2 \mathrm{~cm} / \mathrm{sec}$ \\
Uniform population density & $300 / \mathrm{sq} \mathrm{miles}$ \\
Distance cutoff & $500 \mathrm{miles}$ \\
Shielding factor for ground and cloud dose & $\frac{1}{3}$ \\
Time between end of fissioning and release & $2.5 \mathrm{hours}$ \\
\hline \hline
\end{tabular}

siderably closer to our estimates. The largest remaining discrepancies (of the order of a factor of 2 in the iodine and one-day ground doses) may be attributable to the poorer approximation of the wedge model to the Draft WASH-1400 Gaussian-plume model for the uniquely high deposition velocity assumed for the iodine aerosol.

Most of the assumptions used in the calculation of the average population whole-body dose in Draft WASH-1400 seem to us to be reasonable. We have found one extremely important omission, however, and that is the longterm population dose arising from ground contamination. Draft WASH-1400 apparently neglected the population dose associated with ground contamination after the first day on the grounds that "people will be evacuated from highly radioactive areas" (Appendix VI, p.58).

In fact, as we have already noted, evacuation of areas contaminated above the levels suggested as evacuation thresholds in Draft WASH-1400 would reduce the large population dose associated with ${ }^{137} \mathrm{Cs}$ by less than $10 \%$. The remaining ${ }^{137} \mathrm{Cs}$ ground dose neglected in Draft WASH-1400 would alone still exceed the average reference accident whole-body population dose calculated there by more than a factor of 20 . If we assume no decontamination, complete evacuation of 10000 square miles for 10 years might reduce the total exposure by only a factor of 2 , as we have noted above. We have discussed this question in Section V.F for the important case of ${ }^{137} \mathrm{Cs}$.

\section{Consequences of population doses}

The quantification of biological effects following radiation exposure is a subject full of uncertainties. Some of these uncertainties are due to the intrinsic difficulty of the subject. Thus it has not been established whether the probability of cancer induction or genetic damage increases linearly with dose at the level of tens of rems with the same coefficient that has been measured at hundreds of rems. The large sample size required at this dose level makes experiments impractical, even with small animals. Furthermore, in the case of cancer induction, it is not understood how to make quantitative predictions of human effects on the basis of animal ex-

TABLE XIII. Comparison of our estimate of average population doses with corresponding Draft WASH-1400 results for the reference accident (millions of man-rem).

A. Total whole-body doses

\section{Our results}

$\begin{array}{ll}\text { Inhalation dose } & 3.1 \\ \text { Cloud dose } & 0.19 \\ \text { 1 day ground dose } & 1.1 \\ \text { Long-term ground dose } & 80 .\end{array}$

B. Contributions to whole-body doses from particular elements

\begin{tabular}{|c|c|c|c|c|}
\hline \multicolumn{5}{|c|}{ Our results } \\
\hline Element & $\begin{array}{c}\text { Inhalation } \\
\text { (1 day) }\end{array}$ & $\begin{array}{r}\text { Ground } \\
\text { (1 day) }\end{array}$ & Cloud & Total \\
\hline $\mathrm{Kr}$ & -- & -- & 0.017 & 0.017 \\
\hline $\mathrm{Sr}+\mathrm{Ba}$ & 0.96 & 0.03 & 0.006 & 1.0 \\
\hline $\begin{array}{r}\mathrm{Ru}+\mathrm{Mo}+ \\
\mathrm{Tc}+\mathrm{Rh}\end{array}$ & 0.019 & 0.012 & 0.002 & 0.033 \\
\hline $\mathrm{Te}$ & 0.314 & 0.076 & 0.015 & 0.405 \\
\hline I & 0.613 & 0.89 & 0.08 & 1.581 \\
\hline $\mathrm{Xe}$ & -- & -- & 0.027 & 0.027 \\
\hline $\mathrm{Cs}$ & 0.715 & 0.082 & 0.014 & 0.811 \\
\hline $\mathrm{Ce}$ & 0.137 & 0.000 & 0.000 & 0.137 \\
\hline $\mathbf{P u}$ & 0.294 & 0.000 & 0.000 & 0.294 \\
\hline
\end{tabular}

C. Lung doses
Draft WASH-1400

(Draft WASH-1400, Appendix VI)

$\begin{array}{lr}2.9 & 2.9^{\mathrm{a}} \\ 0.09 & 0.16^{\mathrm{a}} \\ 0.13 & 0.46^{\mathrm{a}}\end{array}$

\begin{tabular}{cccc}
\multicolumn{2}{c}{ Our result } & \multicolumn{2}{c}{ Draft WASH-1400 result } \\
$(30$ day $)$ & $(50 \mathrm{Yr})$ & $(30$ day $)$ \\
21.1 & 31.9 & 15 & 15 \\
\hline
\end{tabular}

a Corrected for programming errors and a factor 2 increase in the Draft WASH-1400 ground dose coefficients (Appendix VI, p. 60) (NRC, 1975b). 
periments. The latent periods of radiation-induced lung and thyroid cancer in humans, for example, are of the order of 10 years. This is the same order as the life expectancy of dogs and much longer than that of the mice, rats, and hamsters which are commonly used for animal experiments. To our knowledge, it is not understood how these latent periods scale from species to species.

Risk estimates must currently be based on those (fortunately few) human experiences where a substantial number of basically healthy individuals have been exposed to relatively high levels of radiation. These include the Hiroshima and Nagasaki survivors, uranium miners, radium dial painters, the Marshallese who were exposed to fallout from the 1954 "Bravo" H-bomb test, and various groups of individuals who were exposed to high doses of radiation a few decades ago for treatment of noncancerous conditions before the medical community fully realized the limitations and the hazards of such treatments. Because the statistics are small, the exposures uncertain, and the followup incomplete, it has been impossible to establish any but the roughest cancer dose-response coefficients from these experiences. For genetic effects, even this has been impossible; and risk estimates are based on extrapolations from animal data.

Draft WASH-1400 computes long-term effects on health (cancer induction and genetic impairment) on this same man-rem basis, using the hypothesis of linearity between radiation dose and response. One of the basic documents in this field, the National Academy of Sciences Report; "The Effects on Population of Exposure to Low Levels of Ionizing Radiation" (1972), states:

At this time, the linear hypothesis, which allows the mean tissue dose to be used as the appropriate measure of radiation exposure, provides the only workable approach to numerical estimation of the risk in a population. Further, since there is no means at present of determining the value of the dose-effect slope in the low-dose region of interest, use of the linear extrapolation from data obtained at high doses and dose rates may be justified on pragmatic grounds as a basis for risk estimation. (NAS 1972, p. 89.)

The other basic document in the field (U.N., 1972, p. 431) states:

The Committee wishes to reemphasize that all the estimates apply to short-term exposures at high dose rates and, as discussed in Annex G, are likely to be overestimates of the risk percent dose that may result from protracted irradiation at low dose rates and with low-LET radiation.

In contrast to the uncertainties concerning the induction of cancer and genetic defects, we believe that other uncertainties relating to the acute short-term biological effects of ionizing radiation could be more readily resolved. For example, there appears to be great uncertainty concerning the thresholds for acute effects following the inhalation of fission products and on the dependence of these thresholds on beta ray energy. These uncertainties in acute effects could be substantially re- duced by a limited number of experiments using large mammals.

In our calculations, we used the same standard references for cancer and genetic dose-effect coefficients as were used by the authors of Draft WASH-1400; namely, the National Academy of Sciences (1972) and the United Nations (1972) studies referred to above.

3. Summary of results and comparison with draft WASH -1400

The results of our analysis of the consequences of the WASH-1400 reference accident (PWR2) are summarized and compared with those in Draft WASH-1400 in Table XIV. The two calculations differ in the following important respects:

(i) Draft WASH-1400 does not calculate the dose received from ground contamination after the first day. We have calculated the full integrated dose received to 500 miles, using the fallout-dose prescription adopted in the U.N. Committee Report (see above) for cesium and not altering any other assumption made in Draft WASH-1400, and find that the total whole-body dose to the population would be increased by a factor of about 25.

(ii) Draft WASH-1400 use the figure 100 cancer deaths per million whole-body man-rem (Appendix VI, p. 53) based on the NAS committee estimate of 50 to 165 for the first 25 to 27 years after radiation. We use the figure of about 130 cancer deaths per million man-rem, based on the extrapolation of the NAS results taking into account the age distribution of the population, latent period, etc. This change, when coupled with the factor of 25 increase in whole-body dose to the population, results in an estimate of about 10000 long-term cancer deaths due to whole-body exposure, as opposed to the 310 calculated in Draft WASH.

(iii) Draft WASH-1400 does not calculate the additional cancer death risk from the lung and thyroid tissue exposed to high doses of beta radiation but calculates cancer risk only from the average whole-body dose. We estimate the additional number of lung and thyroid cancer deaths produced by beta radiation. For lung cancer, we use dose-exposure coefficients extrapolated from the 30-day lung-dose-exposure coefficients given in Draft WASH-1400. The extrapolation is not large, as explained in Appendix $\Pi$. Table XIII shows a 30-day lung dose of 21.1 million man-rem. For the lung and thyroid cancer dose-effect coefficients, we use the ranges given in the NAS report. In the case of thyroid cancer deaths, we supplement the above procedure as follows: (See Appendix II):

- We use the AEC and EPA exposure-dose coefficients for children as the two ends of a range of uncertainty;

- To allow for the possibility argued in Draft WASH1400 that beta radiation from ${ }^{131} \mathrm{I}$ may be up to ten times less effective than $x$ rays in inducing thyroid cancer, we multiply the bottom end of the range of uncertainty of the associated NAS dose-effect coefficient by a factor of 0.3 (in view of the contribution from other radioiodines-see Table XXXVIII); 
- We take the dose-effect coefficient to be equal in adults and children;

- We use National Cancer Institute thyroid cancer relative mortality statistics;

- We count only cancer deaths which occur during the first 30 years after exposure (See Appendix II).

On the above basis, we find approximately 600-1600 additional lung cancer deaths and 500-4000 additional deaths from thyroid cancer which were not calculated in the Draft WASH-1400 numbers.

(iv) While Draft WASH-1400 does not explicitly estimate morbidity due to thyroid nodule cases, using the assumptions in Draft WASH -1400, we use the wedge model to calculate that there would be approximately 25000 cases. In view of the biological uncertainties involved, however, we also calculate a range of values for this effect that change the results implicit in Draft WASH -1400 by a factor of 0.9 to 12 . This change results in a range of $22500-300000$ thy roid nodule cases instead of the $\mathbf{2 5 0 0 0}$ cases implicit in Draft WASH-1400 (our estimate). See Appendix $\Pi-3 . b$ and Appendix $\Pi-5$ for detailed discussion of the complex problem of thyroid doses and effects. (v) Draft WASH-1400 uses the figure of 100 genetic defects per million whole body man-rem. We interpret the National Academy of Sciences report used by Draft WASH-1400 to estimate the following ranges of genetic consequences per million whole-body man-rem: 25-250 identifiable dominant genetic effects, 12.5 noninheritable genetic defects, and 0-500 additional constitutional or degenerative diseases. Taking the first two effects into account, we use a range of 40-250 genetic defects per million whole-body rem (or a correction factor of 0.4 to 2.5 in the Draft WASH-1400 figures). This change, when coupled with the factor of 25 increase in whole-body dose to the population, results in an estimate of 3000 to 20000 genetic defects instead of $\mathbf{3 1 0}$ genetic defects calculated in Draft WASH-1400.

The interpretation of these results should be approached with some caution. It is essential to recognize not only the tentative nature of consequence assessment, in light of uncertain data, but also the different values which might be attached to the risks and consequences of an accident by individuals and by society. If this particular accident (which is assigned a probability of order $6 \times 10^{-6}$ per reactor year in Draft WASH-1400) should occur, the probability that an individual in the

TABLE XIV. Effect of changed assumptions on Draft WASH-1400 reference accident average man-rem consequences.

A. Cancer Deaths

$$
\text { Assumption change }{ }^{\text {a }}
$$

1. Truncation of ground dose at one day...to... no truncation

2. 100 cancer deaths $/\left(10^{6}\right.$ whole body rem). . to. . . (about 130$) /\left(10^{6}\right.$ whole body rem).

3. Neglect of cancer induction from lung dose. . to... (20-50)/ $\left(10^{6}\right.$ lung rem) over 40 years following exposure

4. Neglect of deaths from beta-ray induced thyroid cancer... to... $(12-75$ thyroid cancers $) /\left(10^{6}\right.$ thyroid rem $)$ over 30 years following exposure, use range of AEC-EPA conversion factors, assume $4 \%$ mortality for persons exposed as children, $15 \%$ for persons exposed as adults.

B. Morbidity

Not calculated in

Draft WASH-1400

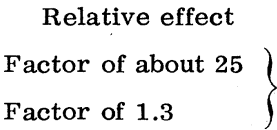

Not calculated in

Draft WASH-1400
1. Show full range of uncertainty of thyroid exposure-dose and dose consequence coefficients

Factor of $0.9-12.0$

$22500-300000$ thyroid nodule cases instead of 25000 (our estimatenot explicitly stated in Draft WASH-1400)

\section{Genetic Defects}

1. Truncation of ground dose at one day... to... no truncation

2. (100 genetic defects $) /\left(10^{6}\right.$ whole-body rem $) \ldots$ to. .. (25-250) identifiable dominant genetic defects and 12.5 noninheritable genetic defects $/\left(10^{6}\right.$ whole-body rem). Not included are $0-500$ additional constitutional or degenerative diseases $/\left(10^{6}\right.$ wholebody rem)

$\left.\begin{array}{l}\text { Factor of about } 25 \\ \begin{array}{l}\text { Factor of } 0.4-2.5 \text { in } \\ \text { genetic defects }\end{array}\end{array}\right\} \begin{aligned} & 3000-20000 \text { genetic } \\ & \text { defects instead of } 310 \\ & \text { genetic defects }\end{aligned}$

10000 cancer deaths instead of 310 cancer deaths

Add 600-1600 lung cancer deaths during following

Add 500-4 000 thyroid cancer deaths in 30 years following exposure

a ... indicate assumption change from. .. to. .. .

$\mathrm{b}$ It is essential to note that these incremental cancer deaths and morbidity would occur over natural lifetimes of a very large exposed population. These calculations are based on a population density of $300 / \mathrm{mi}^{2}$ which results in an exposure of a population of about 10 million. A population density of $165 / \mathrm{mi}^{2}$ would multiply all total consequence estimates by 0.55 (See Footnote, page $\mathrm{x} \times \mathbf{x x})$. Independent of assumed population density, there would be an additional risk of cancer to the average exposed individual of one chance in a thousand, with the risk distributed over a substantial fraction of his natural lifetime. 
exposed region (a 0.25 radian sector of radius 500 miles) will die during his natural lifetime of cancer induced by his exposure is, on the average, one chance in a thousand $(0.1 \%)$ according to our results. This individual risk would be spread over many years following the assumed accident, with the cancer manifesting itself during the decades following exposure.

Average property damage cost estimates are given in Draft WASH-1400 for the reference accident as $\$ 1.8$ billion. Our analysis of this problem is less detailed primarily because an adequate breakdown of these costs is not given in Draft WASH-1400. It appears, however, that a substantial fraction of these costs is associated with long-term land contamination. This cost of land denial is assumed in Draft WASH-1400 to increase linearly with the duration of contamination above the thresholds indicated in Table $\mathrm{X}$ up to a maximum of ten years, after which the loss is assumed to equal the total value of the land. This part of the damages would have to be increased by approximately an order of magnitude if the $U$. N. values for the contamination halflives of ${ }^{90} \mathrm{Sr}$ and ${ }^{137} \mathrm{Cs}$ were substituted for those used in Draft WASH-1400.

In conclusion, although some of the uncertainties cited above may be resolved in the favor of small effects, it appears that the long-term consequences of a postulated meltdown accident with failure of the containment would be much larger than indicated by the Draft WASH-1400, primarily because of the influence of contamination by ${ }^{137} \mathrm{Cs}$ and the large organ beta dose. The reference accident release is calçulated in Draft WASH-1400 to occur in six percent of all meltdown accidents and it is argued there that this probability could be reduced still further by relatively simple measures. However, similar considerations to those we have calculated above for the reference accident consequences would also apply to the calculated consequences for other meltdown release categories.

\section{J. Mitigating the consequences of a large release of radioactivity}

There are many ways in which the consequences of possible reactor accidents such as the above might be reduced. Draft WASH-1400 discusses three on which we have already commented: evacuation, diversion of contaminated food stuffs, and decontamination. In addition, we have emphasized the possible benefits of controlled release from the containment in case of overpressure, with resulting filtration, instead of uncontrolled containment failure. 'There are three additional possibilities for mitigating consequences which we believe deserve investigation:

\section{1. lodine blocking}

Thyroid damage could well be numerically one of the largest contributors to impaired health and mortality from a major reactor accident. A Civil Defense study (See Appendix II) has concluded that the uptake of radioiodines by adult thyroid can be reduced by an order of magnitude by taking a $100 \mathrm{mg}$ "blocking" dose of potassium iodine (KI). The Civil Defense study asserts that enough KI pills to protect the entire population of the United States for a week could be stockpiled for a cost of the order of $\$ 1$ million. The adverse side effects are described as minor. This option, which is also noted in Draft WASH-1400 (Appendix VI, p. 36), would appear to be well worth exploring.

\section{Air filtration}

The practicality of installing effective emergency aerosol air filters in the air intakes of large buildings should be explored. Such air filters could substantially reduce the population inhalation dose and the problem of decontaminating the interiors of these buildings in case of a large nuclear accident. The possibility of stockpiling effective emergency breathing masks for the population within a few tens of miles of reactor sites should also be considered. Such a measure could reduce substantially the acute effects of a meltdown accident even if evacuation proved to be impossible or too slow.

\section{Siting policy}

Distance from important population and agricultural areas can be considered as another dimension in the "containment" of a large radioactive release. We find that the distances involved in the calculation of manrem consequences are potentially so great that it would be difficult to find sites in the U. S. which would surely reduce them by an order of magnitude. However, it might be possible to site reactors so remotely that the probabilities of individuals suffering adverse consequences becomes small. If water contamination in a meltdown accident has serious consequences, as we believe may be the case, this hazard should also be evaluated in siting decisions in the same manner earthquakes and other potential dangers are taken into account.

In evaluating the problem of mitigation of consequences, particular attention should be given to evacuation and decontamination. Since contamination by relatively long-lived isotopes such as ${ }^{137} \mathrm{Cs},{ }^{90} \mathrm{Sr}$, and plutonium is potentially by far the greatest source of total population dose, the total population dose could be greatly reduced if effective decontamination techniques could be implemented. Similarly, the land area that would have to be evacuated for a long time could also be greatly reduced.

At present we feel there should be a better assessment of the natural decontamination time scale (especially of built-up areas) and of possible decontamination techniques, time scales, and costs. Information is needed on these aspects not only for decontamination of land but also for buildings. A much more careful analysis of possible evacuation thresholds and agricultural diversion thresholds is desirable.

A comprehensive strategy involving a selective combination of evacuation and decontamination procedures may make it possible to reduce considerably the health and economic consequences of a major release of radioactivity. 


\section{THE LIGHT-WATER REACTOR SAFETY RESEARCH PROGRAM}

\section{A. Introduction}

The preceding portions of the report have been concerned with a review and evaluation of the technical issues that directly influence the safety of LWRs. As we turn to an examination of the safety research program, it is useful to highlight the principal observations that have special significance for safety research. Our examination of possible accident-initiating events (Sec. IV) focuses on the integrity of the primary system, on system transients, and on initiation through possible lapses in quality assurance and through human actions, accidental or intentional. Our report next examines what is known about the course of an accident and the functions of various safety systems intended to control the accident (e.g., the ECCS) or mitigate its consequences (e.g., the containment system). With respect to reactor behavior during a LOCA with ECCS operation we find that much has been learned about the behavior of individual system components under a range of conditions, so that a conservative prescription of individual component behavior can be made in many instances. There is also considerable qualitative understanding of the behavior of the system as a whole. However, we are not convinced by the present experimental data and calculational methods that a complete quantitative assessment of transient behavior and LOCA can be made at present. Both the data base and the calculational codes are inadequate for such an assessment.

With regard to containment, we find that containments have worked well in controlling routine and minor radioactive emissions, but have not yet been subjected to definitive test by a major accident or large-scale experiment. The containment might be breached under sufficiently severe accident conditions, with consequences that are difficult to calculate with any accuracy, based on present information, but which we estimate could be severe in extreme cases.

It is clear that a great deal of research, development and quality control effort has been and continues to be directed toward safe design and operation. Although the overall safety record has been good, we find that it is certainly possible, albeit unlikely, for accidents to be initiated. However, it is very difficult to quantify accurately the probability of such occurrences on the basis of available information. Many aspects of the problem need to be better understood to make this possible.

Thus we view the safety research program to be of great importance, since its function is to resolve these issues and uncertainties and to lay the groundwork for improvements. The $\mathrm{AEC},{ }^{16}$ principally the Division of Reactor Safety Research (RSR), has an extensive program in progress. This effort is supplemented by research programs of the individual vendors and other independent organizations, especially the Electric Power Research Institute (EPRI) with its growing sponsorship of safety research in the nuclear field. We will concen-

\footnotetext{
${ }^{16}$ See Footnote 1.
}

trate on the research program of RSR, which is the most important because of its scope, size, and openness to public scrutiny. We will introduce information about other efforts where it is available and appropriate.

Research concerning reactor safety dates back to the 1940 s. Throughout the 1950 s and 1960 s it was included as an important responsibility of AEC divisions carrying out reactor research and development. The creation of RSR in May 1973, answered the need for an organization within the AEC that is divorced from development activities and centers its efforts exclusively on the research needs of reactor safety. In the words of its director, Dr. Herbert J. C. Kouts, in an article in Nuclear Safety (1974)

\begin{abstract}
"[RSR] was created because of the need for an AEC program on reactor safety research which is (1) clearly responsive to stated needs of the Regulatory Staff and of the Advisory Committee on Reactor Safeguards and (2) free from any appearance of conflicts of interest deriving from a simultaneous development responsibility. Our mission is primarily to implement this program and secondarily to provide the Commission with an assessment capability in reactor safety independent of both the developmental and regulatory responsibilities."
\end{abstract}

The RSR program on LWRs is based on four main parts:

(1) Separate effects tests, intended to give an engineering understanding of the response of the various LWR components and subsystems over wide ranges of imposed conditions.

(2) Computer code development, combining the engineering response of the components with the basic engineering and physics equations, including the equations of hydraulics and thermodynamics, in order to predict the response of the complex LWR system to hypothetical accident conditions.

(3) Integral system experiments, smaller in scale than a full LWR, but hopefully large enough to test the ability of the codes to predict system effects accurately under a range of LOCA and transient accident conditions.

(4) Primary system integrity experiments and tests, intended to improve understanding of the integrity of the primary reactor system and its ability to withstand a range of operating and accident conditions.

We will examine the strengths and possible deficiencies of this program. At the outset one should note that the research program has several goals, which sometimes conflict. In the short term, it seeks to provide to the AEC Office of Regulation (REG) an improved basis for safety evaluation of LWRs. Since previous work has not yet developed codes capable of fully predicting the course of an accident with accuracy, RSR has tried to meet this short-term goal by the development of "conservative" computer codes, in which phenomena are modeled in a way intended to overestimate the severity of undesirable effects.

The long-term goal of the research program is to be able to understand and predict those aspects of reactor behavior that are relevant to safety in a convincing quantitative manner. For this purpose, RSR is develop- 
ing advanced computer codes which are intended to give a more "realistic" calculation through more accurate treatment of results of the separate effects tests and more realistic modeling of physical phenomena. An expanded effort is now underway to study the important separate effects more realistically, usually with the aid of special facilities. Noteworthy examples are the Large Plenum-Fill experiment being conducted at the Pacific Northwest (Battelle) Laboratory under RSR sponsorship and also large LOCA heat transfer experiments.

When such an advanced code is developed, its predictive ability needs to be tested on a series of integral systems tests which hopefully will demonstrate its validity in a complete quantitative manner. The currently planned test series is centered on the LOFT (Loss of Fluid Test) facility located at the Idaho National Engineering Laboratory at Idaho Falls. LOFT is a $55 \mathrm{Mwt}$ reactor intended to simulate on a scaled basis the conditions of a $3300 \mathrm{Mwt}$ PWR representative of current large designs.

In this research program there are a number of difficult technical problems to be resolved: the design of separate effects tests with the appropriate physics and chemistry; the design and verification of complex, large-scale computer codes using information from separate and integral effects tests; the extrapolation of such "verified" calculations to understand nonlinear phenomena on the much larger scale of a commercial LWR; and the study of accident containment, consequence estimation, and accident mitigation and recovery.

Fortunately, in the formation and management of RSR the AEC has demonstrated increased sensitivity to the need for better understanding of these issues by the scientific and technical community. Again, quoting from Dr. Kouts' Nuclear Safety (Kouts, 1974) article,

"Most people who are apprehensive [about nuclear reactors] do not have the background to understand the technical issues and react to the opinions of those who supposedly do understand. The only way to settle their concerns is to convince the scientific and technical community and make the case perfectly clear to it. If we cannot convince the general scientific community that nuclear power is safe, we cannot hope to convince the populace at large."

RSR has been very cooperative in providing our group with access to whatever information we needed for our evaluation of the safety research program. In the sections which follow, a detailed review will be presented of each of its major parts. We then present our overall evaluation of the program and consider several alternative programs for the future. Finally, organizational and management issues are addressed.

\section{B. Program resources and emphases}

The resources for work directly related to light-water reactor safety come mainly from the sources indicated in Table XV. A detailed breakdown of the RSR budget for FY74-75 and the proposed Congressional Submittal for FY76 are shown in Table XVI. The corresponding EPRI resources estimated for reactor safety research
TABLE XV. Budgets for work related to LWR safety (in millions of dollars)/FY is fiscal year; CY is calendar year.

\begin{tabular}{lcc}
\hline \hline & 1974 & 1975 \\
\hline AEC/RSR & $42(\mathrm{FY})$ & $53(\mathrm{FY})$ \\
EPRI & $17(\mathrm{CY})$ & $36(\mathrm{CY})$ \\
Vendors & & $\sim 12$ \\
\hline \hline
\end{tabular}

are shown in Table XVII. The largest fraction of the RSR budget is allocated to integral systems tests, mainly LOFT. Another considerable amount, $\$ 4.2 \mathrm{M}$ in FY74 and $\$ 5.3 \mathrm{M}$ in FY75, is allocated in conjunction with the Power Burst Facility (PBF) for the purpose of testing scaled fuel elements under transient conditions. Studies of primary system integrity are budgeted at $\$ 1.6 \mathrm{M}$ in FY74 and $\$ 2.8 \mathrm{M}$ in FY75. Other separate effects tests represent $\$ 9.0 \mathrm{M}$ for FY74 and grow to $\$ 14.4 \mathrm{M}$ in FY75. Finally, the safety assessment for new reactor technologies in RSR received $\$ 1.5 \mathrm{M}$ in FY74 although it is budgeted at $\$ 5.5 \mathrm{M}$ in FY75. Additional funds are budgeted for safety-related research for advanced reactors with AEC contractors (now under ERDA) carrying out development.

It is enlightening to break the total 1975 resource allocation, including estimates from EPRI and industry, into the four safety categories discussed in this report. Approximately $\$ 100 \mathrm{M}$ total is budgeted. When roughly $\$ 29 \mathrm{M}$ is removed for "product improvement," we estimate the amount for safety research shown in Table XVIII.

The RSR budget breakdown clearly indicates the dominance of the LOCA issue and the pressures to respond to REG's need for better evaluation tools. Very little is devoted to research on better means of containment or to the estimation of accident consequences or to means for accident recovery. As can be seen from Table XVII, EPRI has instituted a growing safety research program related to LWRs and their technology. This is a new and rapidly evolving program covering a wide variety of topics ranging from system studies to an examination of pump and valve technology. We have the impression that emphasis is placed on topics of current concern to the power industry (e.g., diagnostics, earthquake studies) as well as on important topics of physics and material science that are involved in today's problems (e.g., zircaloy-steam oxidation kinetics). Some attention is also given to topics (e.g., plutonium recycle) of a long-range nature. The EPRI programs are coordinated with those of RSR by their respective managements, including complementary funding of particular aspects of some programs. These topics and budgets will continue to evolve both in scope and detail, so the reader should regard this information on EPRI programs as representative but not definitive. We will return to the resources question when we address program balance in our Overall Evaluation of the Safety Research Program, Sec. VI.J below.

\section{Research to improve primary system integrity}

The discussion in Sec. IV which centered on the primary vessel, major piping, and the steam generator, 
TABLE XVI. Reactor safety research program (in millions of dollars).

\begin{tabular}{|c|c|c|c|}
\hline & $\begin{array}{l}\text { Revised } \\
\text { FY } 1974\end{array}$ & $\begin{array}{l}\text { Mid-year plan } \\
\quad \text { FY } 1975\end{array}$ & $\begin{array}{c}\text { Congressional submittal } \\
\text { FY } 1976^{\mathrm{a}}\end{array}$ \\
\hline \multicolumn{4}{|l|}{ LWR exper. \& analysis } \\
\hline Primary system integrity & $\$ 1.6$ & $\$ 2.8$ & $\$ 4.9$ \\
\hline Semiscale & 3.3 & 2.8 & 3.6 \\
\hline Separate effects & 9.0 & 14.4 & 18.6 \\
\hline LOFT experimental program & 17.3 & 12.9 & 14.3 \\
\hline PBF experimental program & 1.6 & 2.5 & 3.6 \\
\hline Code dev. \& application & 1.5 & 2.5 & 3.6 \\
\hline Subtotal & 34.3 & 37.9 & 48.6 \\
\hline Fast reactor safety assessment & 0.7 & 3.0 & 5.4 \\
\hline Gas reactor safety assessment & 0.8 & 2.5 & 3.6 \\
\hline \multicolumn{4}{|l|}{ Nuclear safety test facilities } \\
\hline LOFT & 3.3 & 6.4 & 7.3 \\
\hline $\mathrm{PBF}$ & 2.6 & 2.8 & 6.2 \\
\hline Inel support & 0.2 & 0.3 & 0.5 \\
\hline Subtotal & 6.1 & 9.5 & 14.0 \\
\hline Total reactor safety research & $\$ 41.9$ & $\$ 52.9$ & $\$ 71.6^{\mathrm{a}}$ \\
\hline
\end{tabular}

${ }^{a}$ The FY76 estimates cover the period to July 1,1976 . An additional $\$ 21.0 \mathrm{M}$ is proposed to cover the three month "transitional" period until FY77 begins October 1, 1976.

emphasized the problems of fatigue embrittlement and cracking of pipes, radiation embrittlement, and stress corrosion. In this connection, quality assurance and construction practices are also important. Major areas currently under study are shown in Table XIX, which lists RSR, vendor, and EPRI programs, the integration of which is carried out directly by the staffs of RSRand EPRI. The discussion in Sec. IV stressed the need for additional experimental information on crack initiation and growth. We note that several of the tests listed in Table XIX are concerned with this problem with a predominantly empirical emphasis. Another main recommendation of Sec. IV concerns the importance of developing better methods for in-place nondestructive testing as well as for continuous surveillance and monitoring of acoustic emissions and vibrations that might indicate incipient failure conditions. We note an increasing amount of effort, illustrated in Table XIX, intended to explore

TABLE XVII. EPRI program planning-reactor safety (millions of dollars)/CY is calendar year.

\begin{tabular}{llc}
\hline \hline \multicolumn{1}{c}{ Program area } & CY74 & CY75 \\
\hline Water reactor safety & 6.4 & 6.2 \\
Plutonium recycle & 4.3 & 3.9 \\
Pressure boundary & 2.5 & 3.0 \\
Reliability and diagnostics & 1.5 & 3.6 \\
Fuel and fuel cycle & 1.1 & 3.6 \\
Earthquake and tornado & 0.065 & 1.0 \\
Advanced system & 1.0 & 7.5 \\
Studies and evaluation & $\ldots .$. & 0.6 \\
Operating benchmarks & 0.46 & 1.2 \\
In-service inspection & $\ldots .$. & 3.2 \\
Chemistry & $\ldots .$. & 1.6 \\
Operation and maintenance & $\ldots .9$ \\
& 17.3 & $\overline{36.3}$ \\
\hline \hline
\end{tabular}

and develop such techniques. Research directed toward the integrity of the primary vessel, pipes, and welds appears to be receiving the attention it deserves by the RSR, EPRI, and reactor vendors.

In the case of pumps, valves, and steam generators, it is mainly up to the vendors to satisfy the utilities and the AEC Office of Regulation (REG) that they are making whatever improvements may be needed. Advances in these areas are considered to be product improvement and are covered by other AEC and EPRI sponsored programs, outside the RSR safety research program. Clearly, there is a grey area here with respect to both topics and interfaces; parochial organizational outlooks can easily arise and jeopardize safety through narrow views of responsibilities. The situation would be aided a great deal by better quantification of the failure of various components. Information from quality assurance procedures and from field inspections can and should be utilized to set goals for research or product improvement programs in this vital area.

\section{Separate effects tests}

The discussion of ECCS behavior in Section V provides a detailed examination of the various ECCS phenomena and parameters that are important during a LOCA. The discussion also highlights a number of areas in which

TABLE XVIII. 1975 Resource allocation by safety categories/ FY is fiscal year.

\begin{tabular}{lc}
\hline \multicolumn{1}{c}{ Accident phase } & $\begin{array}{c}\text { Estimate } \\
(\$ \mathrm{M}) \text { FY 1975 }\end{array}$ \\
\hline A-Initiation sequences and prevention & 6 \\
B-Accident phenomena (mainly LOCA) & 65 \\
C-Containment and radiological consequences & 1 \\
D-Recovery & 0 \\
\hline \hline
\end{tabular}


more experimental information is vital in order to improve understanding of the phenomena and to enable description of them over a wide range of conditions. An important part of the safety research program deals with the exploration of these phenomena through study of each of the separate effects.

In this section we will organize the discussion around three time periods used in Section V to characterize LOCA phenomena, namely, Blowdown, Refill and Reflood, and the general group of phenomena common to the whole LOCA. We will characterize what was being studied or planned as of August 1974, along with our impression of the progress likely to be made. Then we will provide an overall evaluation of the separate effects tests along with recommendations concerning their future.

\section{a. Critical LOCA phenomena and separate effects tests}

\section{Group I-General applicability over the whole LOCA (See Table XX)}

- Decay Heat : two new determinations are underway as separate effects tests, which should significantly narrow the present range of uncertainty within the next two or three years.

- Pump Behavior is being studied in two-phase flow over a range of sizes, including some tests with large pumps. Information on scaled-size pumps ( $1 / 3$ and $1 / 5$ scale) will be available within two years. It is very important to get additional information on large pumps over the next few years.

- Fuel and Cladding Problems: zircaloy oxidation and its effects on embrittlement, creep phenomena, gap conductivity, pellet growth, and cracking all need attention and are at least being addressed. Our present understanding should be significantly improved within the next three years by work in progress sponsored by RSR and by EPRI. However, it is likely that many of these problems will need more sustained work. In addition, it is important to carry out the parametric largescale fuel and cladding studies which are planned by RSR over the next five years in the Power Burst Facility $(\mathrm{PBF})$ program (see Sec. VİE).

\section{Group /I-Blowdown time period (See Table XXI)}

- Break flow data have heretofore been limited to size, pressure, and fluid phase conditions quite atypical of a large LWR. It is important to plan better experiments, especially with metastable flow in large-diameter break flows closer to operating conditions. ${ }^{17}$

- Critical heat flux and departure from nucleate boiling are among the most crucial issues during blowdown since they strongly influence the heat carried away before core cooling diminishes. Several blowdown heat transfer experiments are in progress over a variety of conditions and should improve the present limited knowledge, but much remains to be done; LOFT experiments may also prove useful here.

TABLE XIX. Representative studies of primary system elements.

\begin{tabular}{|c|c|}
\hline Primary system element & Representative studies ${ }^{a}$ \\
\hline Primary vessel & $\begin{array}{l}\text { Heavy section steel test program (ORNL) } \\
\text { Fracture toughness tests (vendors) } \\
\text { Radiation effects on vessel matls. (NRL) } \\
\text { Crack arrest in pressure vessel - } \\
\text { steel (BCL) } \\
\text { Crack arrest tests (W) }\end{array}$ \\
\hline Pipes & $\begin{array}{l}\text { Fracture tests (Univ. of Washington) } \\
\text { Fracture tests (BCL) (GE) } \\
\text { Coolant pipe rupture study (GE) } \\
\text { Pipe whip studies (GE) }\end{array}$ \\
\hline Steam generator & $\begin{array}{l}\text { Stress corrosion cracking of steels } \\
\text { and inconel (Ohio State) } \\
\text { Stress corrosion cracking (GE) }\end{array}$ \\
\hline Nondestructive testing & $\begin{array}{l}\text { Vibration monitoring (vendors) } \\
\text { Acoustic emission vessel surveillance } \\
\quad \text { (vendors) (PNL) } \\
\text { Acoustic inspection during welding }\end{array}$ \\
\hline $\left.\begin{array}{l}\text { Pumps } \\
\text { Values } \\
\text { Steam generator }\end{array}\right\}$ & $\begin{array}{l}\text { A variety of studies treated as product } \\
\text { improvement and conducted outside research } \\
\text { programs. (See text.) }\end{array}$ \\
\hline $\begin{array}{l}\text { Acronyms for sources of representative } \\
\text { ORNL-Oak Ridge National Laboratory } \\
\text { BCL-Battelle Columbus Laboratory } \\
\text { Ohio State-Ohio State University } \\
\text { PNL-Pacific Northwest (Battelle) Lab. } \\
\text { NRL-Naval Research Lab } \\
\text { GE-General Electric } \\
\text { W-Westinghouse }\end{array}$ & e studies shown here are: \\
\hline
\end{tabular}

\footnotetext{
${ }^{17}$ Some information may be obtained at Marviken (Sweden) on a BWR; however, its conditions are quite different from those typical of U. S. BWRs, and the applicability is not clear.
} 
TABLE XX. Current separate effects tests-general applicability over whole LOCA.

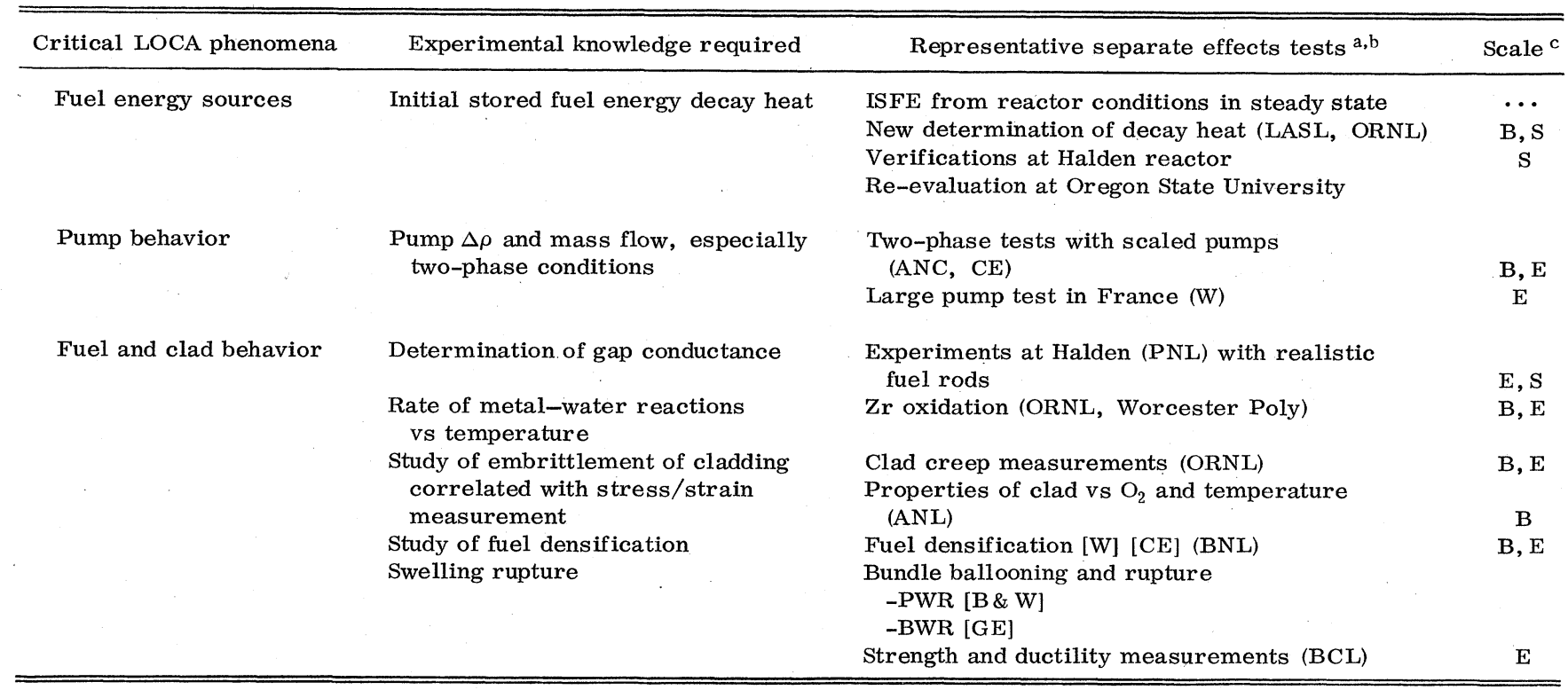

a Symbols used with representative separate effects tests indicate the following:

( ) -Open (publishable) test

[ ]-Proprietary test.

b See Table XXIII for key to contractors carrying out studies as indicated.

c Scale symbols are as follows:

B-Basic model development scale

E-Engineering test scale

S-System verification scale.

- Frictional pressure loss in two-phase flow is now receiving some attention, but more may be needed.

- Lower plenum fluid entrainment and ECC fluid bypass: These involve flow up, down, and around the downcomer in the presence of steam. The present understanding is limited to conditions far from those typical of LWRs. The experiments planned or now in progress should provide a much better understanding for basic models. Especially important is the Large Plenum-Fill experiment, which provides a test of engineering correlations where some three-dimensional effects can be examined. Again, LOFT experiments may also be enlightening.

- Core Distortion and Flow Distribution effects, especially with cross flow and blockage, remain to be addressed satisfactorily as separate effects tests.

\section{Group III-Refill period (See Table XXII)}

ECC fluid penetration: This phenomenon (i.e., the end of ECC fluid bypass) marks the beginning of refill. The same group of experiments discussed under Group II apply here and should significantly improve our present limited knowledge of this subject. But until definitive experiments are completed with three-dimensional effects at roughly operating conditions, the important problem of applicability at full LWR conditions will remain to be resolved.

- Steam fluid interactions in two-phase flow have been a difficult problem for a number of years, and the present state of knowledge is poor, especially for non- equilibrium conditions. Steam-water mixing and interaction experiments are planned or in progress, but the significance of these particular experiments is difficult to assess ahead of time. Much more experimental and theoretical work will undoubtedly be needed to be sure of the two-phase hydraulic behavior, perhaps involving special facilities which currently do not exist in the U.S.

Containment back-pressure effects are, of course, design sensitive and are being pursued to some extent by the reactor vendors.

- Heat transfer during refill is mainly a question of understanding core cooling by the steam where convection eddies may be present. No specific experiments are planned as yet, although some information may be gained from LOFT. gible.

- Break flow during this period is essentially negli-

\section{Group IV-Reflood period (See Table XXIII)}

- Break flow: Some questions remain in this time period in influencing steam binding in the presence of containment backpressure effects. However, other problems are dominant during reflood.

- Steam binding: in PWRs this is thought to be a severe and important problem leading to a possibly marginal reflood rate (see Sec. V). In our opinion, the problem is likely to be eliminated only through some kind of design improvement or through the implementation of an alternate ECCS approach. 
TABLE XXI. Current separate effects tests for blowdown period.

\begin{tabular}{|c|c|c|c|}
\hline Critical LOCA phenomena & Experimental knowledge required & Representative separate effects tests ${ }^{a, b}$ & Scale $^{c}$ \\
\hline Break flow & $\begin{array}{l}\text { Flow of high pressure, two-phase } \\
\text { through large opening as a function } \\
\text { of pipe length/diameter ratio }\end{array}$ & $\begin{array}{l}\text { 1. Critical flow test [GE] } \\
\text { 2. Large pipe break (Marviken) at } \\
\text { several hundred psi }\end{array}$ & $\begin{array}{c}\mathrm{E} \\
\mathrm{E}, \mathrm{S}(?)\end{array}$ \\
\hline Critical heat flux (CHF) & $\begin{array}{l}\text { Conditions for departure from } \\
\text { nucleate boiling (DNB); conditions } \\
\text { for return to nucleate boiling; post } \\
\text { CHF heat transfer }\end{array}$ & $\begin{array}{l}\text { PWR blowdown heat transfer (ORNL) } \\
\text { BWR blowdown heat transfer (GE) } \\
\text { Blowdown DNB [W] [B \&W] (CE) } \\
\text { Transient CHF (ANC) } \\
\text { CHF experiments (MIT) } \\
\text { Level swelling [GE] (w/wo core spray) }\end{array}$ & $\begin{array}{l}\quad \text { E } \\
\text { E, S } \\
\text { E, S(?) } \\
\text { E, S } \\
\quad \text { B } \\
\quad \text { E }\end{array}$ \\
\hline Frictional $\Delta \rho$ & Drag-in pipes in two-phase flow & Two-phase $\Delta \rho[\mathrm{GE}]$ (U. of Cinn.) & $\mathrm{B}, \mathrm{E}$ \\
\hline Lower plenum fluid entrainment & $\begin{array}{l}\text { Entrainment and carryout of lower } \\
\text { plenum fluid, as function of } \\
\text { blowdown conditions }\end{array}$ & Large plenum fill experiment (PNL) & $\mathrm{E}$ \\
\hline ECC bypass effects & $\begin{array}{l}\text { Conditions for ECC fluid bypassing } \\
\text { the downcomer and exiting through } \\
\text { the pipe break }\end{array}$ & $\begin{array}{l}\text { Large plenum fill experiment (PNL) } \\
\text { Bypass test (CREARE) } \\
\text { Bypass in small vessels (Dartmouth) }\end{array}$ & $\begin{array}{r}\mathrm{E}, \mathrm{S} \\
\mathrm{B}, \mathrm{E} \\
\mathrm{B}\end{array}$ \\
\hline $\begin{array}{l}\text { Core distortion and flow } \\
\text { distribution }\end{array}$ & $\begin{array}{l}\text { Distortions of core geometry } \\
\text { Cross flow effects inside the core }\end{array}$ & $\begin{array}{l}\text { No experiment equivalent to full scale } \\
\text { (see reflood period) }\end{array}$ & \\
\hline \multicolumn{4}{|c|}{$\begin{array}{l}\text { a Symbols used with representative s } \\
\text { ( )-Open (publishable) test } \\
\text { [ ]-Proprietary test. } \\
\text { b See Table XXIII for key to contract } \\
\text { c Scale symbols are as follows: } \\
\text { B-Basic model development scale } \\
\text { E-Engineering test scale } \\
\text { S-System verification scale. }\end{array}$} \\
\hline
\end{tabular}

- Reflood heat transfer: experiments should provide more accurate transfer coefficients. Again LOFT data should be helpful for PWR situations, especially in testing the applicability of the results of separate reflood heat transfer and ECC fluid carryover work.

- Oscillatory and transient level effects: We note unresolved questions (e.g., chugging) which can potentially arise in several ways, for example, energy exchanges between the secondary system and the main vessel combined with changes in liquid level in the main vessel, or flashing of liquid level to steam as the liquid level reaches hot core elements at the beginning of Reflood. Such effects can potentially be large and nonlinear and are difficult to study except at the systems effects level.

- Core displacement, fuel rod distortion, and blockage effects: these could be important and need study badly; some progress can be expected in additional effects tests, but parametric tests in the Power Burst Facility (PBF) are needed for a more complete evaluation.

\section{b. Evaluation}

Many separate effects experiments are now in progress on ECCS behavior, including a few large-scale ones, and more are in the planning stages. This is a marked improvement over the situation of a few years ago, and many of the sensitive effects that determine the peak core temperatures are being addressed. The limited understanding of several of these topics makes them appropriate candidates for study by members of the American Physical Society. Of special significance are (1) development of a more tractable approach to handle two- and three-dimensional effects in calculations, and (2) the study of the thermodynamics of coupled, nonequilibrium two-phase flow, which is crucial for many phases of ECCS operation and where the present state of knowledge is poor. Both better experiments and theoretical treatments are badly needed.

It is clear that larger separate effects tests, conducted over a wide range of conditions, could be very important in providing the basic understanding and engineering level tests on which more reliable calculations of ECCS behavior can be made. We recommend that more resources be devoted to such experiments, especially for ECC bypass, heat transfer with cross flow, break flow, nonequilibrium two-phase flow, and experiments to examine core blockage. We understand that AEC-RSR and EPRI are seriously considering this in conjunction with advanced code development. Further cooperation and exchange of data with foreign governments is also strongly encouraged. However, it will be several years before information from all these tests will be available and checked by integral system level experiments; this point has implications for the time scale required to obtain more realistic calculational capability and is treated more fully in Sec. VI. J. 
TABLE XXII. Current separate effects tests for refill period.

\begin{tabular}{|c|c|c|c|}
\hline Critical LOCA phenomena & Experimental knowledge required & Repres entative separate effects tests ${ }^{a, b}$ & Scale $^{c}$ \\
\hline ECC penetration of downcomer & $\begin{array}{l}\text { Conditions for ECC fluid to begin } \\
\text { to flow down the downcomer and } \\
\text { begin to fill the lower plenum }\end{array}$ & $\begin{array}{l}\text { Plenum-fill experiment (PNL) } \\
\text { Bypass test (CREARE) } \\
\text { Bypass in small vessels (Dartmouth) }\end{array}$ & $\begin{array}{l}\mathrm{E}, \mathrm{S} \\
\mathrm{B}, \mathrm{E} \\
\mathrm{B}\end{array}$ \\
\hline Steam/ECC fluid interaction & $\begin{array}{l}\text { Interaction of steam with ECC fluid } \\
\text { as it flows into the vessel, important } \\
\text { for both critical flow conditions and } \\
\text { for ECC bypass and downcomer } \\
\text { penetration (need full range of } \\
\text { equilibrium and nonequilibrium } \\
\text { conditions) }\end{array}$ & $\begin{array}{l}\text { Above set of experiments } \\
\text { Steam-water mixing (W) } \\
\text { Steam-water interaction (CE) }\end{array}$ & $\begin{array}{l}\mathrm{E} \\
\mathrm{E}\end{array}$ \\
\hline $\begin{array}{l}\text { Convection-film heat } \\
\text { transfer }\end{array}$ & $\begin{array}{l}\text { Cooling of core by steam w/wo } \\
\text { Convection eddy cooling }\end{array}$ & $\begin{array}{l}\text { Nothing specifically planned (LOFT } \\
\text { data may be useful) }\end{array}$ & \\
\hline Containment back pressure & $\begin{array}{l}\text { Back pressure effects on refill } \\
\text { conditions }\end{array}$ & $\begin{array}{l}\text { Design sensitive-no specific experiments } \\
\text { planned }\end{array}$ & \\
\hline
\end{tabular}

a Symbols used with representative separate effects tests indicate the following:

( )-Open (publishable) test

[ ]-Proprietary test.

b See Table XXIII for key to contractors carrying out indicated tests.

c Scale symbols are as follows:

B-Basic model development scale

E-Engineering test scale

S-System verification scale.

\section{E. Power burst facility (PBF) program}

Some fuel and cladding problems still need careful evaluation. Effects from pellet swelling/cracking and from variations in pellet-fuel rod conductivity can influence normal reactor operation as well as accident evaluation. As discussed more fully in Sec. V, temperatures above $2200^{\circ} \mathrm{F}$ during an accident could result in zircaloy oxidation, possibly giving rise to embrittlement and cladding creep and collapse. Most of these problems are being studied in laboratory or engineering scale separate effects tests proviously described. However, such fuel failure problems need to be studied with scaled rods under both steady-state and transient reactor conditions.

The Power Burst Facility (PBF) Program was initiated several years ago at Idaho National Engineering Laboratory as an integrated experimental and analytical program to answer this need. Experiments on single fuel rods and small clusters were to be conducted using the special PBF reactor, and analytical models were to be constructed using the experimental information, hopefully resulting in a realistic treatment valid over a wide range of conditions likely to be encountered in LWRs. The PBF reactor itself, a water-moderated burst reactor, has been constructed with a core geometry shown in Fig. 10 and having the maximum capabilities shown in Table XXIV.

A wide range of experiments are planned in 1975 and succeeding years, including tests of individual rod properties (examining fuel behavior); tests of rod-coolant interactions (examining gap conductance); and experiments on rod swelling and ballooning, phenomena important for reflood heat transfer and flow blockage questions. These data would complement the separate effects tests on fuel and cladding problems, and the results are badly needed. However, the operation of PBF has so far been limited by an undesirable oscillatory behavior that has been traced to the PBF fuel. This hitch has delayed the start of the test program, though it appears that the problem has now been corrected. It is important to bring these tests to a successful conclusion in a timely way. We recommend that this situation receive full attention with an accelerated schedule if possible. At the very least, efforts should be made to minimize any further schedule slips, so that results will be timely for input to other programs.

\section{F. LOFT and Semiscale integral test experimental programs}

\section{a. Introduction and program objectives}

The LOFT Integral Test Program is the major AEC program to examine the key phenomena of LOCA and the behavior of the ECCS at the system test level. The LOFT system is a PWR of special design to carry out LOCA experiments on a size and scale ( $55 \mathrm{MWt}$ ) intermediate between a laboratory experiment and that typical of a large PWR (3300 MWt). The stated program objectives (Aerojet, 1974; Coplen et al., 1974) are as follows:

(1) Provide data to industry and the AEC for evaluation of analytical methods and test the adequacy of analytical methods used to predict: 
TABLE XXIII. Current separate effects tests for reflood period.

\begin{tabular}{|c|c|c|c|}
\hline Critical LOCA phenomena & Experimental knowledge required & Representative separate effects tests ${ }^{a}, b$ & Scale ${ }^{c}$ \\
\hline Break flow & Influence on steam binding & $\begin{array}{l}\text { Sensitive to design because of containment } \\
\text { back pressure-general engineering infor- } \\
\text { mation may be obtained at Marviken }\end{array}$ & $\mathrm{E}$ \\
\hline Steam binding & $\begin{array}{l}\text { Pump } \Delta \rho \text { under two-phase flow; } \\
\text { Effects of steam pressure on re- } \\
\text { flood for superheated steam and/ } \\
\text { or for steam generator tube } \\
\text { breaks }\end{array}$ & $\begin{array}{l}\text { Design sensitive-can only be studied at } \\
\text { System Test level }\end{array}$ & \\
\hline $\begin{array}{l}\text { Reflood rate } \\
\text { (affected by steam binding) }\end{array}$ & $\begin{array}{l}\text { Effect of system-type conditions } \\
\text { on reflood rate }\end{array}$ & $\begin{array}{l}\text { Design sensitive-nothing specifically } \\
\text { planned-may get some information during } \\
\text { reflood heat transfer experiments }\end{array}$ & \\
\hline $\begin{array}{l}\text { Core displacement and } \\
\text { fuel rod distortion; Flow } \\
\text { distribution }\end{array}$ & $\begin{array}{l}\text { Alteration of core and fuel rod } \\
\text { geometry as a result of thermo- } \\
\text { mechanical distortion; } 3 D \text { flow } \\
\text { effects inside core }\end{array}$ & $\begin{array}{l}\text { No experimental equivalent to full scale } \\
\text { Out-pile multirod bundle tests (ORNL) } \\
\text { (See LOFT discussion) } \\
\text { (See fuel and clad behavior under General) }\end{array}$ & $\mathrm{E}$ \\
\hline $\begin{array}{l}\text { Reflood heat transfer } \\
\text { (affected by steam binding) }\end{array}$ & $\begin{array}{l}\text { Rate of cooling of core elements } \\
\text { during reflood conditions }\end{array}$ & $\begin{array}{l}\text { Reflood heating rate tests } \\
\text { - for PWRs [W] (CE) [B \& W] } \\
\text {-for BWRs [GE] (w/wo core spray) } \\
\text { (See LOFT discussion) }\end{array}$ & E \\
\hline $\begin{array}{l}\text { ECC fluid } \\
\text { (droplet carryover) }\end{array}$ & $\begin{array}{l}\text { Amount of ECC fluid carried out } \\
\text { as droplets during reflood }\end{array}$ & $\begin{array}{l}\text { Reflood heating rate tests } \\
\text { - for PWRs [W] (CE) [BW] } \\
\text { - for BWRs [GE] (W/wo core spray) } \\
\text { (See LOFT discussion) }\end{array}$ & $\begin{array}{l}\mathrm{E} \\
\mathrm{E}\end{array}$ \\
\hline $\begin{array}{l}\text { Oscillatory effects in down- } \\
\text { comer and lower plenums }\end{array}$ & $\begin{array}{l}\text { Oscillatory and transient level and } \\
\text { pressure effects during reflood- } \\
\text { conditions for unstable flow. }\end{array}$ & $\begin{array}{l}\text { Reflood heat transfer measurements con- } \\
\text { ducted by CE at Erlangen, Germany. } \\
\text { Needs examination at System Test Level }\end{array}$ & $\mathrm{E}$ \\
\hline Containment back pressure & $\begin{array}{l}\text { Effect of containment back pres- } \\
\text { sure on reflood rate }\end{array}$ & $\begin{array}{l}\text { Engineering-type measurements at ven- } \\
\text { dors Sensitive to design; no experiment } \\
\text { completely equivalent to full-scale con- } \\
\text { tainment. Tests at LOFT and at Marviken } \\
\text { may give generally applicable information }\end{array}$ & \\
\hline
\end{tabular}

a Symbols used with Representative Special Effects Tests indicated the following: ( )-Open (Publishable) Test; [ ]Proprietary Test.

${ }^{\mathrm{b}}$ Key to contractors carrying out indicated tests:

BNL-Brookhaven National Laboratory

ORNL-Oak Ridge National Laboratory

PNL-Pacific Northwest (Battelle) Laboratory

ANC-Aerojet Nuclear Corporation

MIT-Massachusetts Institute of Technology

BCL-Battelle Columbus Laboratory

GE-General Electric

$\mathrm{W}$-Westinghouse

CE-Combustion Engineering

B \& W-Babcock and Wilcox

LASL-Los Alamos Scientific Laboratory

${ }^{c}$ Scale symbols are as follows: B-Basic Model Development Scale; E-Engineering Test Scale.

(a) The transient thermal, mechanical, and nuclear response of the core and primary system components and the hydraulic behavior of the fluid under LOCA conditions.

(b) The capability of current ECCS designs to fulfill their intended function.

(c) The margins of safety inherent in the capability of the current ECCS designs.

(2) Verify the adequacy of the design criteria used to establish the capability of ECCS designs to fulfill their intended function.
(3) Reveal thresholds or unexpected phenomena which affect:

(a) The validity of the analytical models used to predict the thermal, mechanical, and nuclear response of the core and primary system components and the hydraulic behavior of the fluid.

(b) The selection of design parameters pertaining to the capability of ECCS designs to fulfill their intended function.

Accompanying the LOFT Program is the Semiscale Program, which in its present form is a scaled-down 


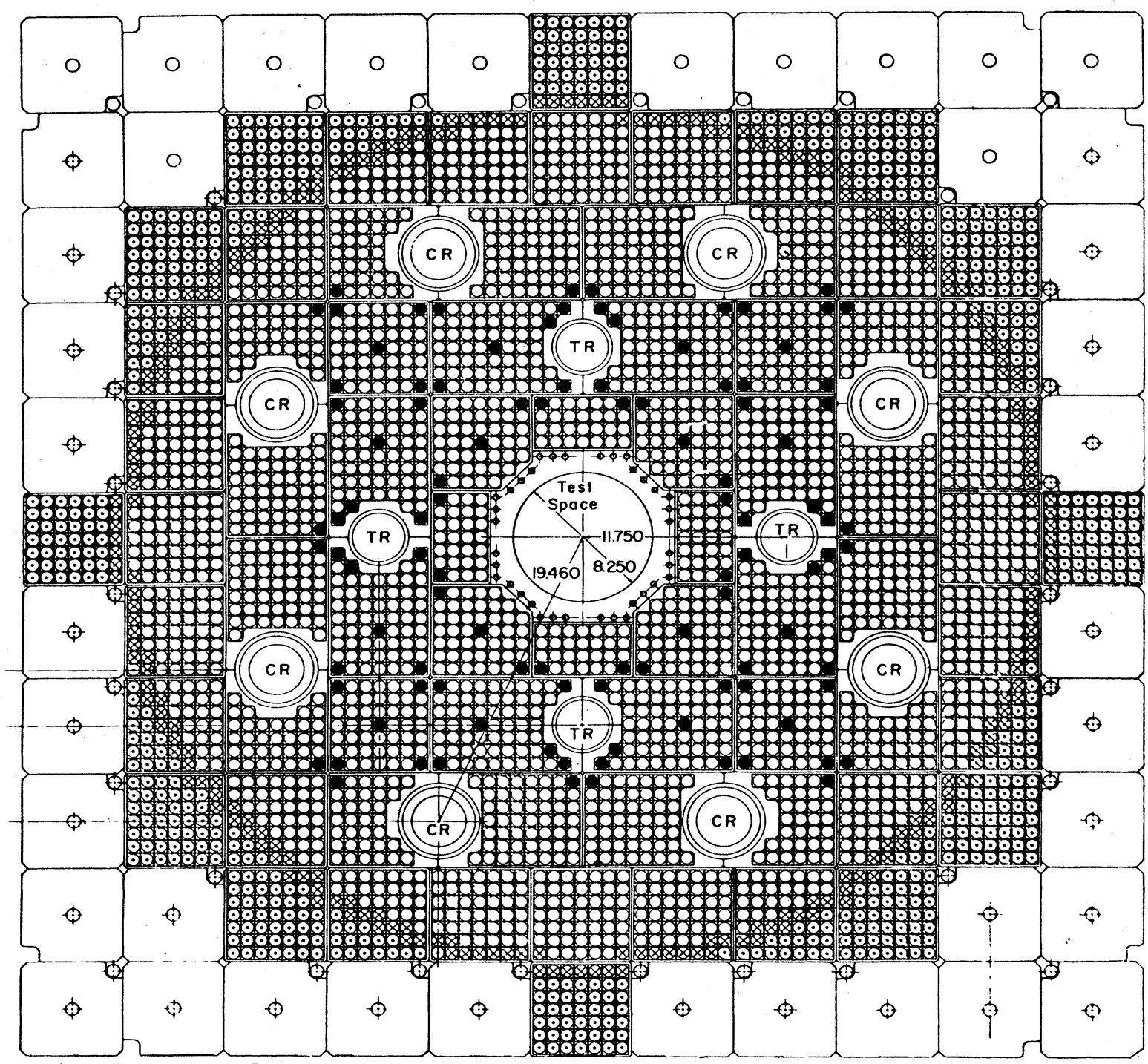

O PBF FUEL ROD STAINLESS STEEL SHIM ROD

- STAINLESS STEEL REFLECTOR ROD

O ALUMINUM DUMMY ROD

FIG. 10. Power burst facility (PBF) core plan view.

\begin{tabular}{lc} 
TABLE XXIV. Maximum capabilities of Power Burst Facility reactor. \\
\hline \hline Maximum core steady power & $40 \mathrm{MW}$ \\
Maximum core power during shaped burst & $1000 \mathrm{MW}$ \\
Maximum core power during natural burst & $240000 \mathrm{MW}$ \\
Minimum asymptotic period during natural burst & $1.3 \mathrm{msec}$ \\
Maximum test cluster size in reentrant in-pile tube & $45 \mathrm{PWR}$ rods \\
$21 \mathrm{BWR}$ rods \\
Maximum test cluster size in through in-pile tube & $\left\{\begin{array}{l}70 \mathrm{PWR} \text { rods } \\
40 \mathrm{BWR} \text { rods }\end{array}\right.$ \\
Maximum loop operating pressure & $250 \mathrm{psig}$ \\
Maximum loop operating temperature & $650^{\circ} \mathrm{F}$ \\
\hline \hline
\end{tabular}


version of LOFT with an electrically heated core in which many thermal and hydraulic phenomena can be studied as separate effects as well as scaled integral effects. Instrumentation can also be tested before the main LOFT experiments.

Thus, when augmented by code analysis, the LOFT test bed makes possible experiments in which all the LOCA phenomena, studied elsewhere as separate effects, are intended to appear and interact with each other as they would during a LOCA at an operational power reactor. The construction and operation of such a test, with major experimental as well as safety requirements, requires a number of years and consumes a substantial fraction of the RSR budget. In this section we will summarize LOFT's salient features and discuss to what extent we believe it can fulfill its role.

\section{b. System description and scaling}

The LOFT system is designed like a small PWR and is constructed on a mobile test assembly, which consists of the pressurized-water reactor system mounted on a railroad dolly that can be moved between engineering buildings and the special containment (test) chamber (see Figs. 11a and 11b). A cutaway of the LOFT reactor vessel is shown in Fig. 12. An electrical core is also available for the initial test series. A schematic layout of LOFT is shown in Fig. 13 where the reader should note that the "operating" side has one steam generator and pressurizer and a pair of pumps. The "break" side has resistive simulators for the pumps and steam generator which connect to a blowdown tank. While there is a general similarity between the LOFT and typical large PWR configurations, one should realize that for a single leg break a PWR usually has several operating (unbroken) loops instead of one and in the loop containing the break the PWR has real components whose properties are not purely resistive. Table XXV shows some important LOFT system parameters.

The crucial issue is the comparison between LOFT system parameters and those typical of a PWR. One notes from the above table that the initial pressures and temperatures are typical of PWR operation. In Table XXVI the volume distributions and volume/power distributions are compared. A more extensive volume breakdown is provided in Table XXVII; surface area comparisons and hydraulic resistance comparisons are presented in Tables XXVIII and XXIX.

A useful examination of the scaling features has recently been completed (Ybarrondo et al., 1975). The classical approach is to consider the appropriate dimen sionless numbers that characterize the problem, such as the Prandtl number (heat exchange), the Mach number (velocity), the Reynolds number (inertia and drag), the Froude number (inertia and gravitational forces), and the length-to-diameter ratio. It suffices to say that complex, transient, two-phase flow is not well enough understood to develop a useful scaling criterion on this basis. Indeed the scaling laws for some of the important phenomena (e.g., critical heat flux) are not understood.

Thus an expedient but less rigorous procedure has been used. To insure that the same relative amounts of fluid are available for energy exchange in LOFT as in a large PWR, the scaling criterion chosen is volumetric and volume/power (i.e., power density) scaling. In addition, scaling of fluid channels and fuel diameter in the core is 1:1. The break area for LOFT is chosen so that the LOCA time scale should be approximately the same in each case tested. Core volume/vessel volume ratios are also made comparable, so that gross corevessel energy transfer effects should also be representative. On these bases, the designers of LOFT believe that its behavior will be comparable with a typical PWR. However, surface-to-volume ratios cannot be matched at the same time; one also cannot match hydraulic resistances, as can be seen from Table XXX. The core area/break area ratios also cannot be matched, and the vessel-volume/system-volume ratio differs by roughly a factor of two. While arguments have been presented (Ybarrondo et al., 1975) that these mismatches in scaling do not introduce serious effects, one must realize that LOFT will be qualitatively representative of PWRs in characteristic behavior, but many points are compromises whose effects are hard to estimate at the present state of knowledge. For example, one expects that during the blowdown the fluid pressures should be comparable since the initial temperature distribution would be selected for the test, and blowdown time scale would be made representative by choice of break aperture.

Refill poses a less felicitous situation. The two-inch downcomer width in LOFT, combined with a vessellength/diameter ratio larger than typical of a PWR, makes LOFT downcomer flow relatively more one-dimensional, with the effect that ECCS bypass phenomena and the refill time scale may be atypical. In addition, steam binding phenomena, important for reflood rate, will not be truly representative. Also, break flow conditions, slip flow in pipes, steam-water phase separation, two-phase frictional effects, possible large nonlinear oscillatory behavior, and momentum effects at flow splits and direction changes are examples of the phenomena that are expected to be dependent on size and other dimensionless parameters previously mentioned. It is difficult to estimate their effects accurately. Finally, possible core blockage will escape simulation in LOFT because of planned use of unpressurized fuel. Core cross flow effects are also unlikely to be representative.

Thus in view of these scaling uncertainties, we believe that it is fair to regard LOFT not as a proof test of PWR ECCS behavior, but rather as an important test of our understanding of the various separate effects in interaction with each other, with basic thermodynamic variables and LOCA time scale which will probably be roughly typical of PWRs. For example, LOFT affords an excellent chance to examine the extent to which we understand the crucial critical-heat-flux behavior; LOFT will certainly throw light on ECC bypass phenomena and reflood heat transfer behavior so important in determining the temperature history of the core, even though results may not be completely typical of operational PWRs.

LOFT also provides the type of facility in which a variety of LOCA conditions can be created with tests that can 


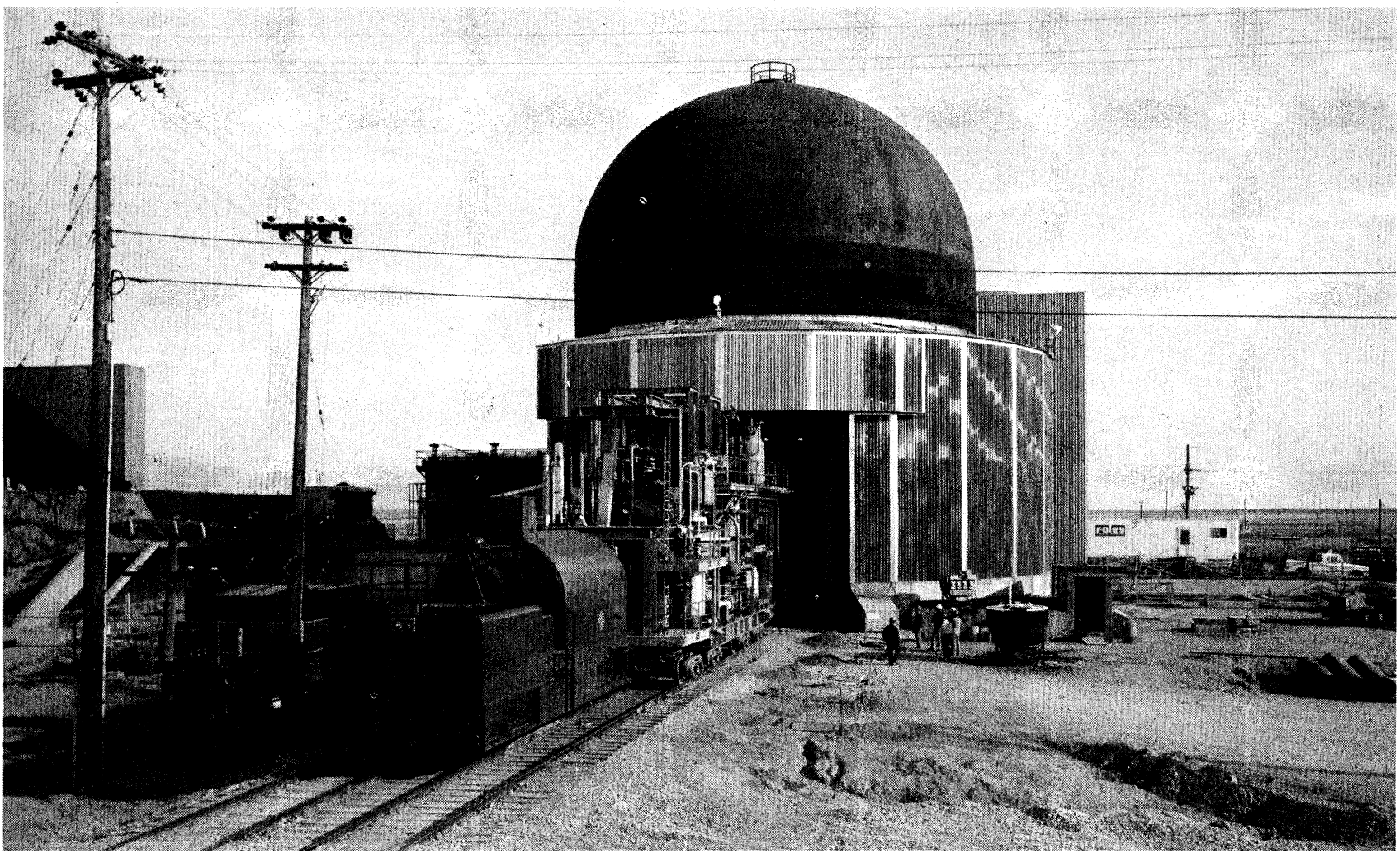

(a)

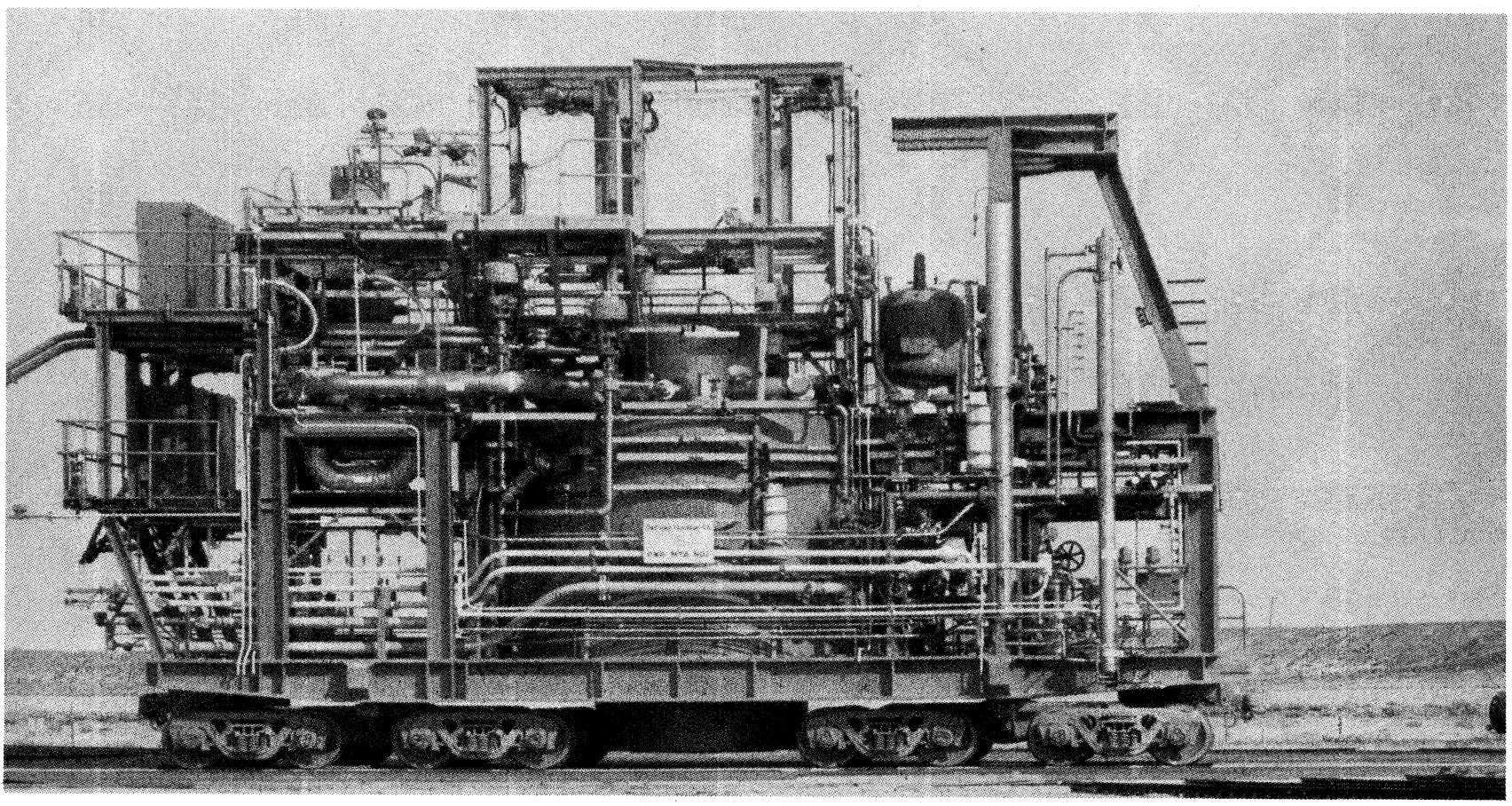

(b)

FIG. 11. (a) LOFT mobile test assembly entering the test chamber. (b) Side view of LOFT mobile test assembly. 


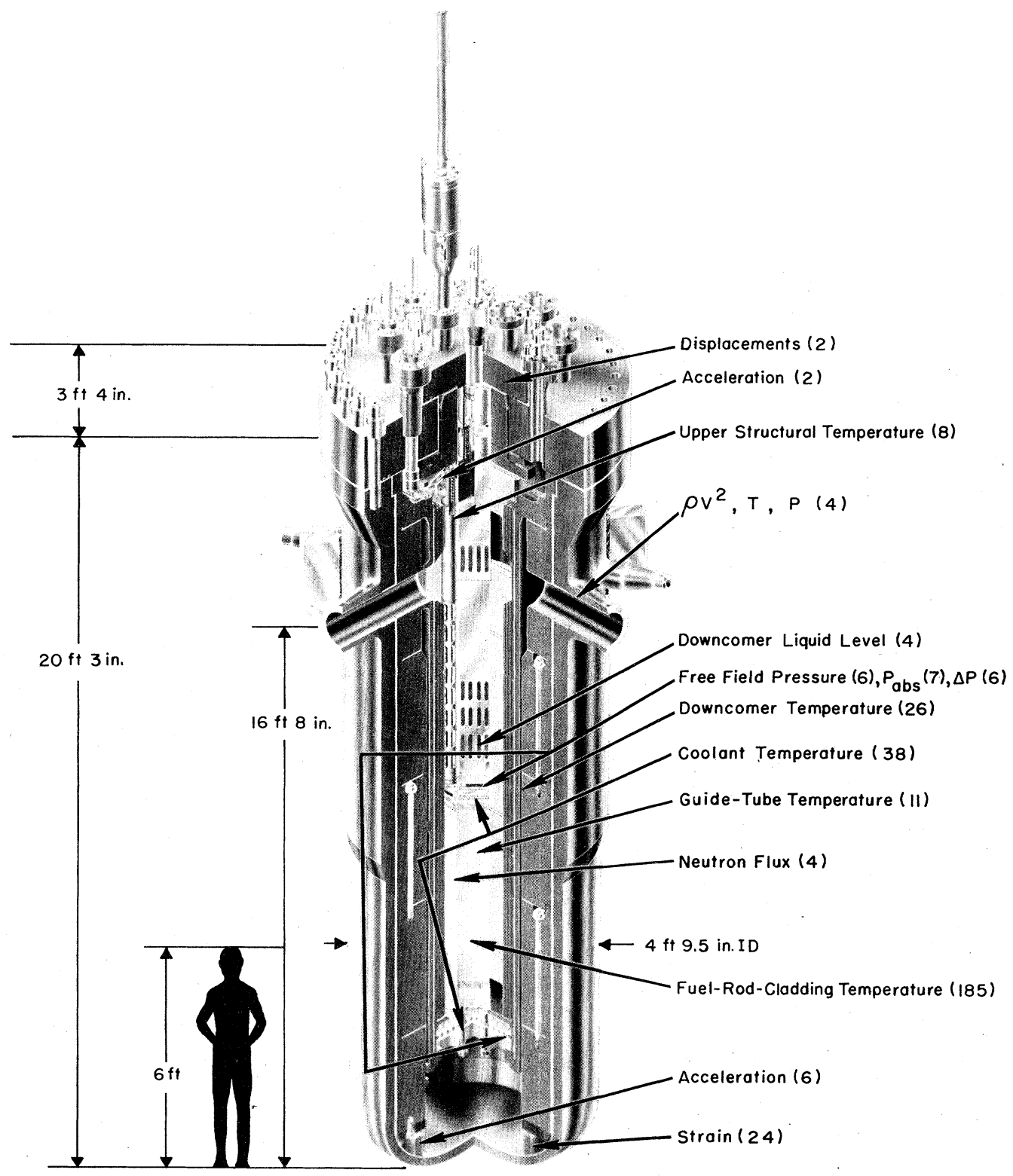

FIG. 12. Schematic of LOFT reactor vessel with associated experimental instrumentation.

take into account previous test results. We strongly urge that a flexible and responsive test approach be maintained. LOFT also provides a unique test bed for study of the efficacy of alternate approaches to ECC (e.g., direct injection of fluid into the lower and/or upper plenum) in comparison with the present approach. We strongly recommend that emphasis be given to such examination of alternate ECCS approaches in LOFT.

In the LOFT planning, one again sees the pervasive nature of the design-basis-accident approach to LWR safety. The LOCA design-basis-accident emphasizes a major pipe break without much reference to other abnormal conditions that could accompany such a break. In the LOFT program this can easily result in a tendency to choose typical normal PWR conditions as initial conditions for the LOFT LOCA, ignoring the possible role of abnormal PWR conditions in inducing the LOCA in an actual situation. Thus we recommend that a wide range of initial conditions, including those corresponding to abnormal LWR conditions, be included in the LOFT series. Such a range is also important to put computer code calculations to a better test.

Because not all the phenomena can be scaled in the same way, LOFT scaling is necessarily a compromise, which makes the issue of code calculation and verification, using LOFT data, extremely complex. As amplified in Sec. VI.I, our evaluation of the present lumpedflow calculations is that they are not likely to provide a satisfactory quantitative comparison of the various separate effects experiments vis-a-vis the same effects 


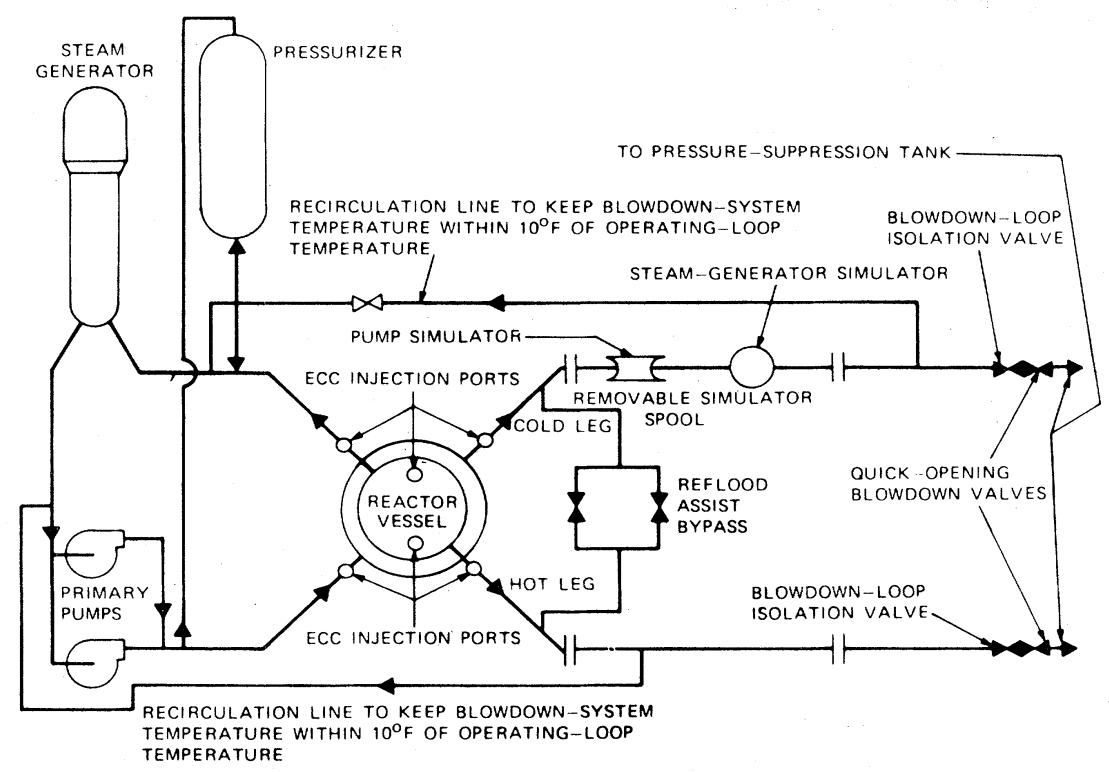

FIG. 13. LOFT primary coolant system with blowdown loop (see Table XXVI). as they occur in LOFT, although with sufficient adjustment of parameters one may get a reasonable fit. We believe that better codes, with a more comprehensive treatment of the physics and incorporating a minimum of arbitrary parameters, are a necessity for this job of careful data comparison. This is particularly true for the more problematic job of scaling such results to full PWR conditions.

Overall, we believe that LOFT can provide a very valuable test of the understanding of PWR LOCA phenomena on an integral system basis, including effects of abnormal initial conditions. Comparisons of the relative effectiveness of alternate ECCS approaches can be of particular value. Yet we have serious reservations about the extent to which one can expect to achieve a complete quantitative understanding of system behavior at full PWR size, based on scaling LOFT tests, even

TABLE XXV. Some important LOFT system parameters.

\begin{tabular}{ll}
\hline \hline Nominal full reactor power & $55 \mathrm{MWt}$ \\
Nominal primary coolant pressure & $2250 \mathrm{psia}$ \\
Average primary coolant temperature & $543^{\circ}$ to $577^{\circ} \mathrm{F}$ \\
$\begin{array}{l}\text { No-load system generator pressure } \\
\quad \text { (secondary side) }\end{array}$ & $930 \mathrm{psia}$ \\
Maximum reactor vessel outlet & \\
$\quad$ temperature & $610^{\circ} \mathrm{F}$ \\
Reactor core length & $5.5 \mathrm{ft}$ \\
Peak linear power density & $19 \mathrm{~kW} / \mathrm{ft}$ \\
Typical number of fuel rods & 1300 \\
Effective core diameter & $\sim 30 \mathrm{in}$ \\
Reactor vessel total volume & $92 \mathrm{ft}^{3}$. \\
\hline \hline
\end{tabular}

Rev. Mod. Phys., Vol. 47, Suppl. No. 1, Summer 1975 given currently planned separate effects tests and advanced codes. It is also sobering to realize that nothing comparable to LOFT exists for the BWR reactor configuration. We observed in Sec. $V$ that there is greater confidence that the ECCS in a BWR will function as designed because of reduced complexity in the primary system compared to a PWR and because of an ECC fluid injection procedure that eliminates steam binding as a problem in BWRs. One must realize, however, that without a system level test, BWR analysis rests heavily on the same type of code calculations whose limitations we have discussed at length. We will return to all these points in our overall evaluation in Sec. VI.J.

\section{c. Instrumentation and measurements}

All of the above discussion has assumed an ability to make all the desired measurements on LOFT to the desired accuracy. It is now appropriate to describe what may be expected from the program. We will summarize the situation as of August 1974, when the detailed study was carried out describing both core measurements and primary system hydraulic measurements. A list and evaluation of measurements for the two regions is presented in Table XXX.

Core measurements: The core has been instrumented with 185 thermocouples to determine the cladding temperature at various core locations and at many positions along rods. There are 11 more thermocouples on guide tubes, and 35 more to measure coolant temperature in representative locations. For pressure determinations there are four measurements of absolute pressure at different locations, and measurement of differential pressure at two locations. Vertical mass flow measurements are attempted by an instrument consisting of a drag disk, to measure $\rho v^{2}$, and a turbine meter, to measure velocity. Core liquid level 
TABLE XXVI. Comparison of LOFT and PWR primary coolant system volume distribution and volume/power ratios.

\begin{tabular}{|c|c|c|c|c|c|}
\hline Item & Component & \multicolumn{2}{|c|}{$\begin{array}{l}\text { Fraction } \\
\text { of total volume }\left(\mathrm{ft}^{3} / \mathrm{ft}^{3}\right) \\
\text { Four- } \\
\text { loop PWR }\end{array}$} & \multicolumn{2}{|c|}{$\begin{array}{l}\text { Volume/power } \\
\text { ratio }\left(\mathrm{ft}^{3} / \mathrm{MWt}\right) \\
\text { Four- }\end{array}$} \\
\hline 1. & Reactor vessel & 0.380 & $0.366^{b}$ & 1.36 & $1.81^{\mathrm{b}}$ \\
\hline 2. & Steam generator ${ }^{c}$ & 0.352 & 0.252 & 1.26 & 1.25 \\
\hline 3. & Primary coolant pump $\mathrm{d}$ & 0.026 & 0.037 & 0.09 & 0.18 \\
\hline 4. & Pressurizer & 0.147 & 0.125 & 0.53 & 0.62 \\
\hline 5 . & Intact loop (s) volume & $0.355^{\mathrm{e}}$ & 0.340 & 1.27 & 1.68 \\
\hline 6. & Ruptured loop volume from reactor vessel to break plane & 0.118 & 0.170 & 0.43 & 0.82 \\
\hline $\begin{array}{l}\text { a } \text { A se } \\
\text { b Base } \\
\text { C Tota } \\
\text { d Tota } \\
\text { e Thre }\end{array}$ & $\begin{array}{l}\text { ed, typical design. } \\
\text { n downcomer gap of } 2.0 \text { in. } \\
\text { lume in all steam generators. } \\
\text { lume in all primary coolant pumps. } \\
\text { four loops in PWR including the three steam generators }\end{array}$ & & & & \\
\hline
\end{tabular}

detectors, which were originally planned, have been deleted. Finally, neutron flux is measured by four fixed power measurements and four scanned power measurements (as function of core axial position).

Our assessment of core instrumentation is that the temperature measurements utilize an appropriate technique and are reasonably sufficient in number. There will clearly be difficulty in accurate calculations of heat transport for the fuel rods without direct measurement of conditions inside the rods. Since the present rods are unpressurized to avoid ballooning and rupture, the gap conductance must be evaluated separately. PBF tes ts are scheduled to study these problems. With unpressurized rods there is risk of collapse during the LOCA cycle, which could create an uncertain thermal conduction geometry that could make reflood heat transfer measurements hard to interpret. While these problems are recognized, we have seen no data that convinces us that changes in fuel rod gap conductance and rod geometry will not be significant. More work needs to be done in this area to insure interpretable quantitative measurements.

The pressure measurements appear adequate in number. However, neither carefully analyzed calibration data nor an accurate determination of errors was available as of August 1974. Thus we view this as a matter to be clarified as soon as possible. The absence of direct core water level measurement is disturbing since a direct check could be made against the time variation of the temperature measurements. One also loses a direct measurement of core reflood oscillations. We gather that such measurements were originally planned, using the technique described below for the other primary system locations, but were dropped for "safety reasons." The AEC should seriously consider their reinstatement. Neutron flux measurements appear sufficient in number and in range; but, as with the pressure measurements, no carefully analyzed calibration data, hence no careful error determination, was available to us. It should be possible to get reasonable neutron measurements with the measurement techniques planned.

The mass flow measurements, using drag disks and turbine meters, pose questions of accuracy and range.
For use in the core, they may give semiquantitative results of vertical flow over roughly a factor of 10 in velocity, provided calibration taking into account shadowing effects shows the gauge useful (see below). There are still major reservations concerning its use in two-phase flow. Again, the absence of carefully analyzed calibration data and an absence of careful error analysis do not reassure us of the performance of this type of measurement, given the importance of mass flow to the testing of our understanding of core hydraulics.

Primary System Thermal Hydraulic Measurements: The measurements to ascertain the important hydraulic variables that characterize the ECCS behavior occur in many locations and are not as easy to summarize as those for the core. Rather than going point by point over the system, we will present our conclusions for each type of measurement that is planned, along with some recommendations concerning some that are not planned at present.

There are many thermocouple locations at critical places in the system with concentration at the core inlet, the downcomer, the core outlet, and individual thermocouples in the upper plenum, steam generator, and at ECC fluid inlet and outlet locations. The choice of locations is a reasonable one. Calibration and time response data have not been processed in detail, and there is some doubt about ability to make good measurements with superheated steam; but with careful calibration and treatment of systematic errors, the prospects for useful temperature measurements are good.

With the pressure measurements (absolute and differential) there are potential problems. First, while there are many individual locations covered, there are many important points where only one gauge is located. In some cases it may be possible to estimate the pressure from other measurements, but one must conclude that there is insufficient redundancy in this basic thermodynamic measurement to insure that the data on each test can stand as a complete, hopefully overdetermined package. More gauges should be available for intercomparison, preferably with ranges which differ significantly enough to act as a proper check on each other and allow for the unexpected. Second, there may be 
TABLE XXVII. Comparison of LOFT and a large PWR (3411 MWt) primary coolant system volume distribution.

\begin{tabular}{|c|c|c|c|c|c|c|}
\hline \multirow{2}{*}{\multicolumn{2}{|c|}{ Item }} & \multicolumn{2}{|c|}{ PWR (Trojan) } & \multicolumn{2}{|c|}{ LOFT } & \multirow[b]{2}{*}{$\begin{array}{l}\text { "Ideal LOFT } \\
\text { volume" }\left(\mathrm{ft}^{3}\right.\end{array}$} \\
\hline & & Volume $\left(\mathrm{ft}^{3}\right)$ & $\%$ total & $\%$ total & Volume $\left(\mathrm{ft}^{3}\right)$ & \\
\hline \multicolumn{7}{|c|}{ 1. Reactor vessel } \\
\hline & a. Outlet plenum & 1959 & 15.95 & 11.7 & 31.8 & 31.6 \\
\hline & b. Core \& bypass & 920 & 7.50 & 4.0 & 11.1 & 14.8 \\
\hline & c. Lower plenum & 1050 & 8.58 & 9.22 & 25.12 & 16.9 \\
\hline & d. Downcomer \& inlet annulus & 721 & 5.89 & 8.91 & 24.25 & 11.6 \\
\hline & Subtotal & & 37.95 & 33.91 & & \\
\hline \multicolumn{7}{|c|}{ 2. Operating loop ${ }^{a}$} \\
\hline & a. Hot leg pipe & 237 & 1.94 & 4.88 & 13.28 & 3.82 \\
\hline & b. Cold leg pipe & 255 & 2.08 & 5.00 & 13.61 & 4.11 \\
\hline & c. Pump suction pipe & 378 & 3.09 & 4.41 & 12 & 6.09 \\
\hline & d. Steam generator & 3231 & 26.4 & 18.2 & 49.4 & 52.1 \\
\hline & e. Pump & 240 & 1.96 & $\underline{2.57}$ & 7.0 & 3.86 \\
\hline & Subtotal & & 35.47 & 35.06 & & \\
\hline \multicolumn{7}{|c|}{ 3. Blowdown loop } \\
\hline & a. Hot leg to break & 79 & 0.65 & 2.27 & 6.19 & 1.27 \\
\hline & b. Cold leg to break & 211 & 1.72 & 2.60 & 7.08 & 3.40 \\
\hline & c. Steam generator & 1077 & 8.80 & 7.12 & 19.39 & 17.3 \\
\hline & d. Pump & 80 & 0.65 & 0.84 & 2.3 & 1.28 \\
\hline & $\begin{array}{l}\text { e. Additional volume- } \\
\text { part of outlet plenum }\end{array}$ & N/A & N/A & N/A & 6.91 & N/A \\
\hline & $\begin{array}{l}\text { part of inlet plenum } \\
\text { padional voiume- }\end{array}$ & N/A & $\underline{N} / \mathrm{A}$ & $\underline{N} / A$ & 7.54 & N/A \\
\hline & Subtotal & & 11.17 & 10.56 & & \\
\hline \multirow[t]{2}{*}{4.} & Pressurizer & 1800 & 14.7 & 12.5 & 34.0 & 29.0 \\
\hline & Total & 12240 & 100 & 100 & 272 & 197 \\
\hline
\end{tabular}

Values for PWR for 3-loops combined.

"Ideal LOFT volume" $=$ PWR volume $\times \frac{\text { LOFT core power of } 55 \mathrm{MWt}}{\text { PWR core power of } 3411 \mathrm{MWt}}$.

serious systematic error problems in such pressure measurements. Many pressure transducers are separated from their orifice by long tubes containing one or two valves, thereby creating possible fluid traps and organ pipe effects that may seriously distort the signals. Such valves were put in for safety reasons which are no longer regarded as so restrictive. The way to fix the measurements seems to be well known but not easy to implement at this stage. Unfortunately, neither carefully reduced calibration data nor a careful error analysis was available in August 1974. Preliminary measurements presented later left serious doubt. It is very important to remedy these difficulties as soon as possible to ensure that credible and accurate pressure measurements can be made.

A relatively nice device has been developed to measure the liquid level and is used at a number of locations in the downcomer and other critical locations. Again, we would like to have seen measurements that assured us of its reliability and ability to respond to oscillations.

A gamma ray densitometer is being utilized to measure density at each of five locations where mass flow instrumentation is also located. The gamma in- strument features a source on one side of a pipe and three detectors at different locations on the other side, so that three line-integrals of density are obtained at the location. The line-averaged density can thus be obtained with response up to $100 \mathrm{~Hz}$. Some information about the type of two-phase flow regime can be obtained by comparing the three detectors at the given location. This technique has proven very useful in other laboratories for two-phase flow measurements. Unfortunately, since neither calibration data nor error analysis was available for the gamma densitometers planned for LOFT, it is impossible to know how accurately the measurements will determine the ratio of steam and liquid densities in typical situations anticipated. This information should be ascertained as soon as possible, since a number of other points in the system would benefit from the measurement, especially if the range can be pushed to low enough densities to obtain values where an appreciable fraction of steam is present.

Mass flow measurements are currently planned at four locations: the core outlet, the downcomer, the blowdown loop hot leg and the blowdown loop cold leg. As mentioned earlier, a drag disk is used to measure 
TABLE XXVIII. Surface area comparison.

\begin{tabular}{|c|c|c|}
\hline Item & LOFT & $\begin{array}{l}\text { Large } \\
\text { PWR }\end{array}$ \\
\hline Core surface area $\left(\mathrm{ft}^{2}\right)$ & 953 & 55630 \\
\hline $\begin{array}{l}\text { Unbroken loop steam generator } \\
\text { tubes }\left(\mathrm{ft}^{2}\right)\end{array}$ & 3530 & $137250^{a}$ \\
\hline Structure area $\left(\mathrm{ft}^{2}\right)$ & $1567^{\mathrm{b}}$ & 67931 \\
\hline Total $\left(\mathrm{ft}^{2}\right)$ & 6050 & 260811 \\
\hline$\frac{\text { Core surface area }}{\text { Actual PCS volumed }}\left(\mathrm{ft}^{-1}\right)$ & $3.5^{\mathrm{c}}$ & 4.54 \\
\hline$\frac{\text { Steam generator tube area }}{\text { Actual PCS volume }}\left(\mathrm{ft}^{-1}\right)$ & 12.97 & $11.2^{\mathrm{a}}$ \\
\hline$\frac{\text { Structure surface area }}{\text { Actual PCS volume }}\left(\mathrm{ft}^{-1}\right)$ & 5.67 & 5.54 \\
\hline$\frac{\text { Total primary surface area }}{\text { Actual PCS volume }}\left(\mathrm{ft}^{-1}\right)$ & 22.2 & 21.3 \\
\hline
\end{tabular}

a Three unbroken loop steam generators only.

b By difference, PWR value includes $48790 \mathrm{ft}^{2}$ for broken loop steam generator.

c If the "LOFT Ideal Volume" of $197 \mathrm{ft}^{3}$ is used, this value is 4.84 .

${ }^{\mathrm{d}}$ PCS is Primary Cooling System.

$\rho v^{2}$ and turbine meter to measure $v$. The gauge as currently designed has already received criticism for its limited range, its accuracy, and the fact that the drag disk and turbine meter shadow each other. In twophase flow it is not obvious how its measurement will be interpreted. We could not find evidence of a careful examination of other mass flow techniques, common in hydrodynamic measurement usage,${ }^{18}$ for possible application to LOFT. In view of the stated desire to test code calculations with such important variables, we believe that effort should be made now, while there is still time, to try to bring to bear additional methods to make point density mass flow and fluid velocity measurements.

Overall, we question whether the LOFT instrumentation is up to the task. Very little serious evaluation and calibration data were available to us as of August 1974 although more has no doubt been accomplished since then. There appear to be serious problems with several of the important measurements, and there is certainly insufficient attention to instrument redundancy and overlap of ranges to allow for the unexpected. In the case of mass flow, we feel that further development is likely to be necessary to obtain satisfactory measurements. We recommend a major effort to upgrade the LOFT instrumentation in quality, range, quantity and redundancy, as time is running short.

\section{d. Semiscale measurements}

Semiscale is currently configured as a 1-1/2 loop "little brother" of LOFT. It is shown in Fig. 14. Its present program objectives center on providing experi-

\footnotetext{
${ }^{18} \mathrm{See}$, for example, G. F. Hewitt, "Role of Experiments in Two-Phase Systems with Particular Reference to Measurement Techniques" (Hewitt, 1972).
}

TABLE XXIX. LOFT blowdown system resistance and a large PWR broken loop resistance comparison for an inlet break.

\begin{tabular}{lcc}
\hline \multicolumn{1}{c}{ Item } & \multicolumn{2}{c}{ " $\mathrm{K}$ " factor } \\
& LOF T & PWR \\
\hline 1. Broken cold leg & 0.071 & 0.043 \\
2. Hot leg to steam generator & 0.12 & 0.087 \\
3. Steam generator & 3.87 & $3.5 \rightarrow 4.00$ \\
4. Steam generator to pump & 0.14 & 0.48 \\
5. Pump locked rotor resistance & 18 & 18 \\
6. Pump to break & 0.76 & 0.071 \\
\hline \hline
\end{tabular}

mental data on LOCA phenomena for basic model development and testing, for LOFT design assessment, and for evaluation of LOFT instrumentation methods. It utilizes an electrically heated core typically 5.5 feet long, of $1.07 \mathrm{MWt}$ maximum, and it is designed to operate up to $2200 \mathrm{psi}$ and $500-600^{\circ} \mathrm{F}$. In a typical (changeable) configuration the vessel is 90 inches in length below the cold leg inlet with an inner diameter much smaller than its length. The downcomer width is typically $1 / 2$ to 1 inch. Thus it has a very one-dimensional appearance and performance relative to LOFT. It is adequately equipped to make the same sort of measurements previously described for LOFT and is well adapted for fairly rapid changes. Transparent vessels can be introduced for special flow observations, but sturdy metal ones are the norm for measurements at high pressure and temperature.

Historically, Semiscale measurements provided the first experimental indications that ECC fluid bypass effects could be important. Measurements of subcooled decompression processes have been useful. Extensive measurements of interactions of injected fluid with twophase fluid have also been important and are continuing in additional configurations. Several series of measurements of critical heat flux phenomena during blowdown have been and are being carried out for correlation with other separate effect measurements conducted

TABLE XXX. Principal LOFT instrumentation.

\begin{tabular}{|c|c|c|c|}
\hline Instrument & Purpose & Core $^{a}$ & $\begin{array}{l}\text { Ptions } \\
\text { Primary } \\
\text { system }^{\text {a }}\end{array}$ \\
\hline Thermocouples & Temperature & OK & $\mathrm{OK}$ \\
\hline Pressure transducers & Absolute pressure & $\cdots$ & M, IR \\
\hline Pressure transducers & Relative pressure & $\mathrm{M}, \mathrm{IR}$ & $\mathrm{M}, \mathrm{IR}$ \\
\hline Gamma ray sensor & Fluid average density & $\cdots$ & $\mathrm{OK} ?, \mathrm{IR}$ \\
\hline Liquid level detector & Fluid level & $\cdots$ & $\mathrm{OK} ?$ \\
\hline $\begin{array}{l}\text { Drag disk }\left(\rho v^{2}\right) \\
\text { Turbine meter }\end{array}$ & Mass flow & $\mathrm{Q}, \mathrm{IR}$ & $\mathrm{Q}, \mathrm{IR}$ \\
\hline $\begin{array}{l}\text { Neutrons-fixed } \\
\text { Neutrons-scanned }\end{array}$ & Neutron flux & OK & $\cdots$ \\
\hline
\end{tabular}

a Comments on Instrumentation:

OK-Instrumentation adequate in type and number Q-Questionable design (significant systematic errors) M-Marginal design (unevaluated possible errors) IR-Inadequate redundancy and/or range OK?-Appears adequate but data on behavior unavailable 


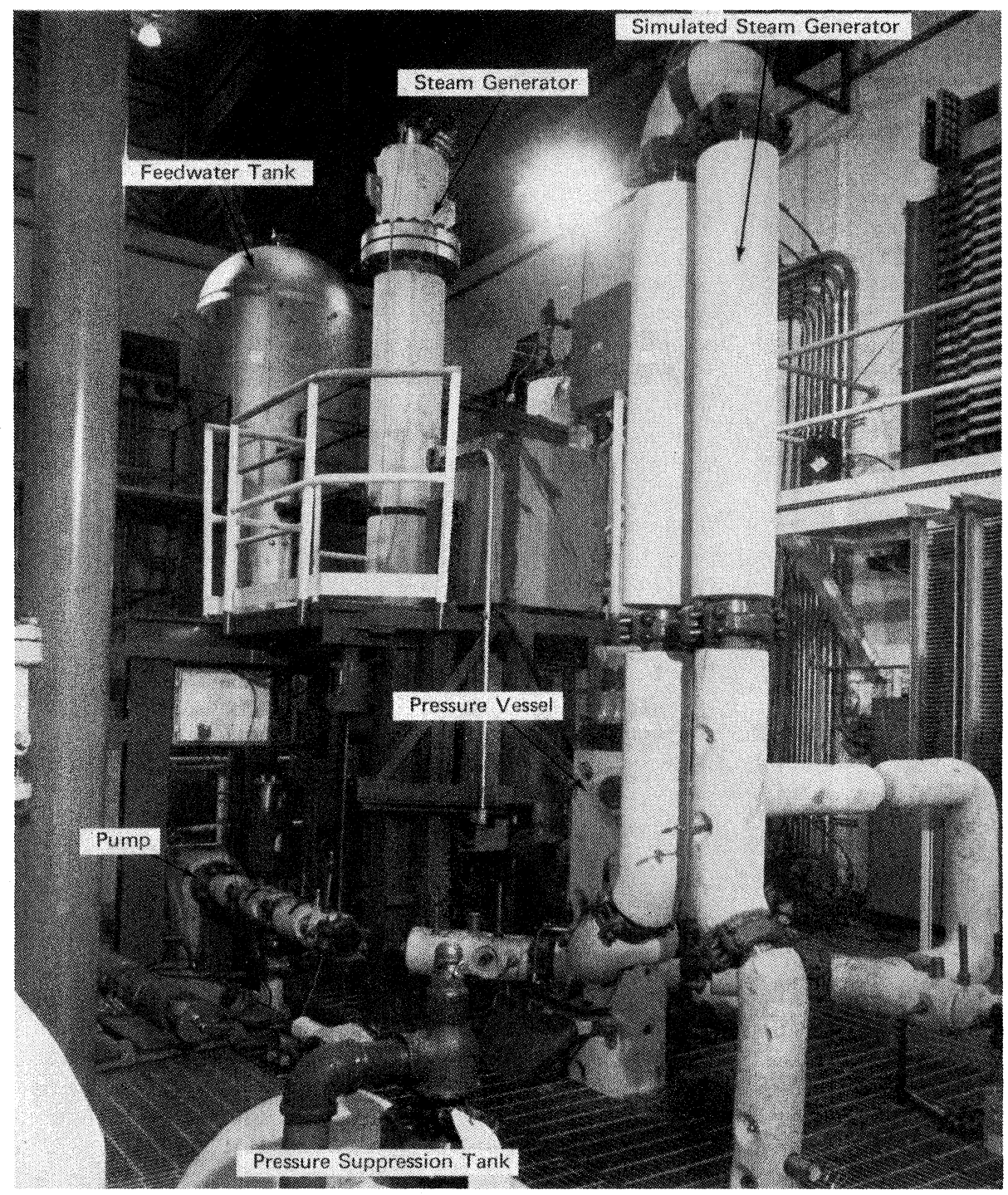

FIG. 14. Thermal reactor safety program: Semiscale Mod-1.

els ewhere. The delay observed in departure from nucleate boiling (DNB) in the Semiscale experiments has already been important in identifying highly desirable potential DNB delay effects in LOFT and by implication perhaps in operational PWRs as well.

Overall, Semiscale's flexibility and adaptability make it an extremely useful tool in studying ECC thermohydraulic behavior, and its instrumentation is sufficient to give code predictions a good test. For example, sensitivity studies of various break locations are a natural for Semiscale. Of course, Semiscale's small size relative to an operational PWR ( 1/3000 in power) and its very one-dimensional character rule out direct applicability of much of its results to operational size reactors. Nevertheless, the program laid out for the next few years, with a variety of blowdown and reflood heat transfer tests, should provide valuable parametric study data when compared with the LOFT results.

\section{G. Containment research}

As part of its safety philosophy of multiple barriers between the public and the radioactivity contained in nuclear reactor fuel in a power plant, the $\mathrm{AEC}$ requires that the entire reactor primary system, and much of the secondary (steam) system, be installed in a large reinforced concrete containment building, along with special systems intended to control radioactivity and maintain suitable building pressure conditions. A brief description is provided in Sec. III and V as important background for the discussion presented here. In normal plant operation, the containment building retains the low-level routine radioactive emissions for processing into holdup tanks for decay before release and for venting through specially designed filters.

In the event of a LOCA, the expelled primary coolant would flash to steam and pressurize the containment 
volume. Containment building design must take into account the maximum temperature and pressure expected from the steam that would be released initially by the primary system. There are often subsystems, using either water or ice, which are intended to quench and condense this steam, along with heat exchangers to control the temperature and hence the pressure over a long term. The heat exchanger must provide for removal of the heat released by the radioactive decay of the reactor fission products, otherwise the heat would increase the steam pressure and might eventually rupture the containment. Condensing the steam, thereby lowering the containment pressure, also reduces the driving force for possible outward leakage. This is important since in a major LOCA the significant radioactivity held in the primary coolant would be released into the containment. There are a number of subsystems, which vary from one reactor to another, intended to control this radioactivity. Among the more important are water sprays containing chemicals to increase retention of radioactive fission products, especially radioactive iodine, and blowers with special recirculation filters and absorption beds to remove or reduce airborne radioactive particles.

There are two possible major complications. First, in a PWR, the LOCA might also release into the containment volume part of the steam contained in the secondary system; this could result in a much higher internal containment pressure than would be the case for steam flashed from the primary system alone. Second, in the unlikely event that the accident is so severe that the core melts, a very large amount of radioactivity would be released into the containment volume, much more than present radioactive removal systems are designed to cope with. Section $\mathrm{V}$ notes that, in the event of actual core melt, there is also the possibility of a steam explosion from interaction of melted fuel and ECCS fluid. Such an explosion could conceivably stress the containment to failure. Under core meltdown, concrete decomposition becomes a possibility leading to even higher pressure inside the containment volume due to noncondensible gaseous products $\left(\mathrm{CO}_{2}\right)$ of the decomposition.

In general, containment systems have worked well in controlling routine and minor radioactive emissions, but have not yet been subjected to definitive test by a major accident or large-scale experiment. It is likely that present heat-removal/pressure-reduction systems and radioactive removal systems would be reasonably effective in ameliorating effects of a minor accident, so that containment would be effective. However, in the event of a major accident, there would be many uncertainties and unknowns concerning pressure and temperature in the containment volume; and there are major uncertainties in the way present heat removal and pressure reduction systems would work. There are also major uncertainties in the effectiveness of systems intended to reduce radioactivity if a severe accident were to occur.

Currently, the main thrust of the U. S. containment research program is in the area of computer codes. AEC RSR is planning to spend about $\$ 150 \mathrm{~K}$ in FY1975 to develop a "best estimate" computer code to model containment behavior during the early and later stages of a loss-of-coolant accident. In FY1976. RSR plans to con- tinue with studies of BWR and ice containment systems.

The vendors are working on containment pressure analysis for their own designs-Westinghouse on the ice condenser system, and General Electric on the BWR double-compartment containment. Westinghouse has also just completed testing a model of their ice condenser system while General Electric is testing a model of one segment of their double-compartment torus design. EPRI was supporting no containment research as of August 1974.

Experimental containment research is taking place in foreign countries. The U. S. has joined with Sweden, Denmark, Norway, Finland, West Germany, France, and Japan in tests at the Marviken power station, originally designed as a $140 \mathrm{MWe}$ boiling heavy-water reactor, located about 100 miles southwest of Stockholm on the Baltic Sea. The nuclear core has never been installed, and steam and heat are supplied by an oil-fired boiler. The U. S. contribution has been $\$ 100 \mathrm{~K}$ for participation in the first set of large-pipe blowdown, containment, and isolation valve tests. The Marviken containment test has had 16 blowdown runs and more are planned to observe the following:

- containment response to ruptures in pipe systems connected to the pressure vessel;

- component behavior under post accident conditions; and

- containment leakage and iodine transport. Marviken is most similar to the General Electric BWR Mark III containment system, but the important link between Marviken and light-water reactors is the data on mass flows of steam and water from a reactor pressure vessel when a pipe bursts and on the subsequent pressure suppression. These data should be useful to check existing computer calculations to see if they give a good representation of containment stresses and response phenomena. Preliminary analysis and reports based on the 16 blowdowns indicate that pressure-suppression systems did work qualitatively as expected. Although the full test reports are not completed, there are several aspects that appear encouraging, including the following:

(1) Preliminary analysis indicated leakage far below allowable levels when minute amounts of radioactive iodine were included in the blowdowns to check on leakage through the concrete containment walls and isolation valves.

(2) The pressure suppression appeared to be adequate. Since the area/volume ratio for the Marviken containment is roughly the same as for the larger U. S. light-water reactors, it will be a relatively straightforward extrapolation to estimate the steam condensation on the drywell walls for small pipe breaks or into the wet well pool for large breaks.

We encourage RSR to publish these data along with an evaluation of their significance to U. S. light-water reactor containment systems. In addition, we encourage the U.S. to pay close attention to future Marviken tests and to the possibility that they may be altered to test containment spray and other improved systems. RSR is presently negotiating to participate in the Marviken II experiments, at a cost of $\$ 250 \mathrm{~K} / \mathrm{yr}$. The U. S. will 
also exchange information with the German containment study being conducted at Battelle, Frankfurt. The Frankfurt effort is on drywell containments with subcompartments and blowdown tests to observe stress on the primary system.

We believe that much more can and should be done with respect to containment research in the U. S. program, emphasizing better methods and subsystems as well as better understanding of the important uncertainties in phenomena that can potentially lead to higher containment pressures. We recommend:

(1) Particular emphasis on research on containment systems immune to loss of power. Some of the worst loss-of-coolant accident scenarios involve loss of power and subsequent loss of the containment functions.

(2) Improvement in pressure suppression and heat removal devices. Once again, the main emphasis should be on systems that are independent and passive.

(3) More research into the mechanism of steam explosions, in particular, behavior of a molten mixture of steel, zirconium, and uranium dioxide when contacted by water. In addition, it is important to understand better the extent to which the containment building and subsystems could resist the effects of a steam explosion should one occur.

(4) Study of whether it might be beneficial to provide relief through a filtered stack (soft failure) should the pressure limit on the containment be reached.

(5) Improvement and better verification of the reliability of filters under abnormal conditions and improvement in the efficiency and reliability of scrubbing sprays, which must remove the bulk of radioactive materials in the event of a major accident.

(6) Re-examination of "core catcher" concepts to augment present types of containment in the event of a meltdown accident (see Sec. VI.H).

Coordination of effort here among vendors, national laboratories, universities, and others can only be achieved effectively by a single agency, clearly the AEC. We note that the present system of incentives does not seem to stimulate much effort in the industry. We believe that a concerted research effort in these areas, with judicious use of engineering hardware and better testing, is likely to be fruitful.

\section{H. Research on radiological consequences, accident mitigation, and recovery}

In analyzing the societal cost-benefit balance of commercial nuclear reactors, one needs to know the probability of accidents as well as the consequences. We have noted that RSR has mounted an internal program to continue the probability analysis utilized in Draft WASH -1400. We addressed in Sec. V the difficulties in estimating the consequences should a major accident occur and containment fail. A principal conclusion of that discussion is that the phenomena important in the consequences and recovery from a major reactor accident are among the least well understood of all those involved in the safety and operation of nuclear reactors.

Immediate deaths from high doses, in the unlikely event of a major accident, are rather localized geo- graphically. Such acute effects are highly dependent on local meteorological conditions and on locations of population centers relative to the reactor; they are also sensitive to release conditions specific to the accident. Moreover, a variety of radionuclides must be considered, and there are many uncertainties in dose-effect coefficients. Thus, for acute effects, siting is a very important consideration. However, many of the expected deaths, illnesses, and property denial in such an accident would be related to total dose (man-rem) effects which are likely to be long term and extend over large distances (hundreds of miles). This category of consequences is less sensitive to vagaries of weather and location, but is quite sensitive to long-term doseeffect coefficients for genetic and cancer effects and to the effectiveness of decontamination, which must contend with radionuclides removed from the air by various fixing mechanisms. The discussion in Sec. $V$ points out that radioiodine in the air would be a principal cause of latent thyroid cancers that would occur over a long time period, and long-lived radioactive cesium, deposited and fixed in the soil, would be very important for land contamination thresholds for evacuation and agriculture withdrawal.

The research program on these phenomena falls into four broad categories dealing with fission product release, meteorology, biological effects, and decontamination effectiveness. The AEC is sponsoring research on fission product chemistry at Oak Ridge ( $\$ 100 \mathrm{~K}$, FY1975) and a study by Sandia ( $\$ 150 \mathrm{~K}, \mathrm{FY} 1975)$ of core meltdown. The AEC is also doing research on the mechanisms of release of important radionuclides (such as $\mathrm{Xe}, \mathrm{Ce}, \mathrm{I}, \mathrm{Te}$ ) from molten fuel. The RSR is negotiating with the West German government for use of a crucible to melt down some two to four kilograms of simulated irradiated nuclear fuel and then actual irradiated fuel to observe the fission product release and measure heat conduction in molten fuel. While this research is a good start, there are other areas that need attention, for example, the effect of $\mathrm{CO}_{2}$ sparging on the molten core as a result of concrete decomposition and the magnitude of volatile and nonvolatile oxide releases.

The $\mathrm{AEC}$ now requires that at each reactor site the licensee must conduct meteorological monitoring and maintain records of conditions at and around the plant. These records are intended to allow analysis of radioactive release consequences on a plant-by-plant basis and to make site selection more quantifiable. What is lacking is long-distance meteorological data regarding bulk air transport for hundreds of miles. While there have been many studies and analyses of atmospheric dispersion and plume behavior for nonradioactive pollutants, even more data would be very useful. We recommend that RSR continue integrating these results and applying them to radioactive releases, taking the initiative to obtain the missing meteorological and dispersion information. Of particular interest are (1) the effects of wind wander and velocity changes with height and time on dispersion over long distances; (2) improved understanding of solubility and particle size in aerosols likely to be released in a core melt accident; 
and (3) the likely deposition rates of these aerosols and other airborne radioactive materials.

Much animal research has been conducted on biological and radiological consequences of various radionuclides and radiations. Unfortunately, not very much of these data is easy to apply directly to the question of accident consequences because of the great uncertainties in drawing conclusions concerning humans based on experiments conducted on animals. Better understanding is needed concerning the extent to which animal data on radiation-induced cancer and genetic effects can be used to predict the corresponding consequences in humans. Many uncertainties in all such areas center on the so far unanswered questions on long term, low-level radiation effects, including possible thresholds and cell damage and repair mechanisms.

The dose-exposure coefficient, which relates inhaled radioactive iodine to thyroid doses, should be understood better, especially for children. Analysis of consequences to the thyroid would be greatly aided by a systematic analysis of all data available on cancer and nodule production resulting from the diagnostic and therapeutic administration of radioactive iodine. For analysis of immediate deaths due to acute radiation damage, a critical question concerning the amount of self-shielding of the gastrointestinal tract against beta radiation $f$ rom different important radionuclides could be answered by experiments on large mammals.

As regards the mitigation of human consequences, research is needed to verify the usefulness of taking ordinary (nonradioactive) iodine tablets to block the thy roid from absorbing radioactive iodine, particularly with regard to the appropriate dose and possible side effects for children. There seems to be little knowledge readily available on the effectiveness of simple face masks for protection from inhalation of radioactive material and aerosols; research and experience at AEC plants and installations may be very relevant here.

Finally, another area of great uncertainty is that of decontamination and accident recovery. Thresholds for evacuation and agricultural withdrawal and plans for eventual reoccupation of contaminated areas all suffer from a lack of definitive knowledge concerning the fixing of radionuclides in the soil, buildings, etc., and their subsequent history. Uncertainty in such factors, especially for radioactive cesium, contributes a substantial portion of the uncertainty in our analysis of long-term consequences, presented in Sec. V.

There are also many questions regarding core melt during an accident followed by penetration through the bottom of the containment. F or example, how much filtering would take place in the overlying soil? Will there be blowouts? Would radioactive gases and material force venting channels to the surface? How much radioactive material would be leached into ground water or nearby surface water? Such questions strongly affect analysis of consequences as well as the significance of possible "core catcher" technology.

We think that the AEC should take action to improve our understanding of these areas and provide a firmer foundation for analysis of accident recovery and for more effective recovery planning.

\section{Research Techniques}

Every scientist knows that in addition to statistical errors, experiments have systematic errors, and that such er rors frequently set the limit of sensitivity of measurement. In a similar way theories (and computer codes) often have built-in errors due to approximations, uncertainties in parameters, and incorrect assumptions that limit their ability to make accurate predictions. To do research well, one must understand the inherent limitations of the techniques being applied. In this section, we shall discuss several of the potential sources of systematic error in the theory and experiments concerned with reactor accidents and how these systematic errors can be minimized.

\section{Relationship between computer codes and experiments}

The naive observer might think that, given the initial conditions (temperature, pressure, characterization of pumps, location and size of pipe break, etc.) an adequate code should be able to compute and to display the course of an accident as if the full-scale experiment were conducted and photographed with good time and space resolution at all internal points. Such a code would allow a single computer run to replace a very expensive experiment. Unfortunately, such an advanced code is not within the present capabilities of computers.

The design for the normal operating modes of power reactors puts no great strain on hydrodynamics calculations by computer. The flows are slow, so that acceleration terms and compressibility play negligible roles. The calculation of the course of a reactor incident following an assumed pipe break or other anomaly, however, is quite a different story. Now, acceleration terms may dominate friction or gravity forces; the flow may be two-phase (mixed or layered steam and water), with different velocities and temperatures for the two phases and with interconversion between the phases, and the flow is typically highly compressible.

The present "best estimate" computer codes were described in Sec. V. They break up the LWR system into several lumped parameter subsystems, each of which is modeled in a simplified, one-dimensional way, using averaged flow field variables, and incorporating a number of arbitrary parameters which must be adjusted. Nobody claims that the present best estimate codes are capable of predicting reactor response in a satisfactory way. More realistic codes require the results of the separate effects tests (including such items as pump behavior and plenum filling times) and more complex computer programming (inclusion of more physically reasonable models of two-phase flow, increase in number of nodal points, characterization of the reactor as a multidimensional rather than as a onedimensional device).

When a more realistic code is written, its predictive ability can be evaluated on a large-scale systems test. The planned test, LOFT, necessarily differs in many ways from a large commercial PWR, as was discussed at length in Sec. VI.F. One of the earliest jobs the LOCA computer codes were asked to perform was to help 
choose the scales of the various elements of LOFT in such a way that the response of LOFT to a loss-of-coolant accident would be as close as possible to the expected response of a PWR. RSR hopes that the advanced computer codes will be able to predict the response of LOFT in some detail and that the LOFT series will thus "verif $y$ " the codes. If the codes can in fact predict the response of LOFT, RSR believes there will be reason to hope that the codes represent a physically accurate simulation of what is happening, and that the same codes, when adjusted to represent a full scale PWR, will therefore be able to predict what happens within such a reactor during an accident.

The symbiotic relationship planned between codes and experiments is, in principle, an excellent one but depends on a delicate balance, so that one must be concerned with the pitfalls that lie on both sides. While these pitfalls are well known within the research community, we point them out here because of the importance of unbiased results in the reactor saf ety program and because of the difficulty of repeating the major experiments in order to provide independent checks.

There is disagreement within RSR as to whether the computer codes will be sufficiently well developed at the time of the LOFT experiments to be able to predict the results. RSR has recently expanded the coding effort in a significant way by initiating code developments at Los Alamos Scientific Laboratory and Brookhaven National Laboratory to attempt a more adequate realistic model, but it is almost impossible to judge the chances for success from the outside. It is encouraging that top people at RSR recognize the importance of having these codes developed in time for LOFT, but RSR appears to us to be preparing a fallback position, to the effect that it will be sufficient if the "evaluation model" codes can make "conservative" predictions of the LOFT experiments. As we explain in the following section, such a "verification of conservatism" may severely degrade the usefulness of LOFT into merely the sum of separate effects tests. If the goals of LOF T are to be lowered in this way, the cost of LOFT should be reconsidered, compared to an expanded program of separate effects tests. A genuine "verification" is critically important to our understanding of LWR safety.

There are limitations to what can be accomplished by computer simulation. In general, for systems sufficiently simple to permit the fundamental equations of physics to be invoked at every step and detailed conformance to these equations (including all phases, three dimensions, and all phenomena) to be required, there is a high probability of accurately modeling the behavior of the system. To the extent that it is necessary to build in experimental and empirical relations, there is inevitably some degradation of confidence in the potential success of the program. A large LWR may be too complex to simulate adequately on present-day computers; and it certainly is too complex, if by "adequately" we require complete three-dimensional two-phase nonequilibrium flow. Thus what an "adequate" approximation is and how difficult it is to achieve lie at the heart of our discussion.

An anecdote may illustrate these points. An experi- ment was being prepared at the SPERT facility in Idaho a number of years ago that differed only in minor ways from an experiment that had been run earlier that week. Since it was late Friday afternoon, the apparatus was set up but not run until the following Monday morning. When finally run, the experiment yielded startlingly different results, which could not be attributed to the minor changes made since the previous run. The differences were finally accounted for: microscopic bubbles, which had formed at the bulk and surface nucleation sites, had had time to dissolve over the weekend, significantly altering the boiling response of the water to sudden depressurization. It is hard to imagine any practical computer code that could have predicted this effect. The conclusions to be drawn are several. The first is the obvious but important conclusion that the result of a single experiment may be very different $f$ rom one repetition to another. The second is that although computer programs can supplement a vigorous experimental program, they cannot supplant it. The codes are best used as an aid to thought, as a means of discovering unexpected phenomena which are inherent in those equations. It is incumbent upon the AEC to study the limitations of their research procedures and to be able to argue the sufficiency of the present code-experiment research program to answer the major questions of. reactor safety. The present approach sounds reasonable, but comparison with previous programs using similar approaches would be helpful in determining the capabilities of the program. The computer code approach may be the best means of predicting the behavior of LWRs. However, there is the danger that the mere existence of extremely complicated computer codes (which few people understand) will lead to an overconfidence in reactor safety, unless the limitations of such codes are clearly understood and explained to the technical community by those who use them.

The "production" codes used at present by the AEC (RELAP-4) and by the manufacturers treat only a locally homogeneous fluid (although they purport to handle compressible flow). Unfortunately, all these codes have errors or ambiguities in the physics of flow through abrupt enlargements of flow path (Zuber, 1974). Present reactor codes schematize a reactor-plus-plumbing system by a finite number (roughly 100) of lumped segments. Since one of these segments may contain or be bounded by a sudden change in geometry (enlargement, contraction, or change of direction), it is important to have an accurate means of deriving volume-averaged conservation equations. Zuber has shown that the existing production codes use an averaged equation which is derived from an inapplicable stream-tube treatment. Five momentum-integrated equations discussed in detail by Zuber actually disagree with one another, but it is impossible to evaluate the magnitude of uncertainty introduced into full-scale LOCA calculations by this treatment of the hydrodynamics, in view of the tendency to introduce adjustment factors ${ }^{19}$ in the process of "maturation" of the code to fit small-scale experiments. These

\footnotetext{
${ }^{19} \mathrm{An}$ example of such a factor is the "break discharge coefficient" used in several of the present codes.
} 
adjustment factors may well fall short of accurately dealing with the large-scale flows. We have no argument with simplifying assumptions that are suitably justified; we do fault incorrect physics and arbitrary parameters.

There is no question that the better approach is to insist on a formal, scientifically justifiable, published derivation of the fundamental equations used in any numerical calculation. This would provide a forum for involvement of government, industry, and university talent and experience over a much broader range of interest than is involved at present.

Apart from its relation to the computer codes, the experimental program has inherent limitations. For example, in the LOFT series as presently planned, the fuel rods will not be pressurized with helium as they are in some LWRs. Thus there is no way that the LOFT program could have discovered the fact that ballooning can be more serious than a higher maximum cladding temperature, since unpressurized cladding doesn't balloon. A second example has to do with the problem of steam binding, i.e., the accumulation of steam in the reactor vessel which can prevent the ECCS from reflooding the core. Steam binding is critically dependent on the behavior of the steam generator and the pumps; a pressure difference of a few $1 \mathrm{~b} / \mathrm{in}^{2}$ can determine whether or not the core is cooled at a sufficient rate. In LOFT these elements in the broken loop are represented by purely resistive elements, so that it is unlikely that steam binding in LOFT can simulate the phenomena of steam binding in a commercial LWR.

All experiments have their limitations, and it is important to recognize what they are. Unless they are made clear whenever the results of LOFT are discussed, the casual observer might mistakenly assume that the LOF T series of experiments will represent in all important respects a LOCA in a commercial light-water reactor.

\section{Verification of computer codes: Double-blind techniques}

It is common practice in medical experimentation for neither the subject nor the experimenter to be aware of the parameters of the experiment until the data have been taken and the procedures for analysis specified. For example, during the testing of a new drug neither the physician nor the patient will know whether the medicine being administered is the true medicine or the control placebo until the patient's response has been recorded. Such experiments are called "double-blind." These procedures are essential to avoid the effect that the expectations of the physician or patient might have on the response of the subject.

The need for double-blind techniques even in physics and engineering research has been clearly demonstrated In nuclear reactor safety research, it is important that the predictions of the code be made before the experimental data are available (of course, using the actual conditions of the experiment in the calculations, not the nominal ones). If the re is disagreement, then the code can be modified for forced agreement, "maturation," a valid. and useful process. However, such a code can be used for extrapolation to a full-size LWR only with grave reservations since its predictive power has not been demonstrated. To verify the "matured" code, it is necessary to test its predictive ability on an experiment that differs substantially from the original experiment.

Although they have not been standard practice, doubleblind procedures have been used on occasion by both the AEC Office of Regulation (REG) and RSR. Clear examples are the "standard problems" used by REG to check the predictive power of the AEC-sponsored and vendor codes, of which there have been four run so far. F or each of these, the experimental results have not been released to the code developers until the predictions of the code were submitted to REG. Then the comparisons between experiment and theory were made by a third party (REG) and made available to the AEC. For the current type of codes used in licensing, REG required only that the conservative predictions be, in fact, conservative. A disagreement in the realistic predictions was used as a probe to indicate where the codes could be improved.

RSR also used double-blind techniques by requiring that predictions from realistic model codes be submitted prior to all experiments. Of course, for the procedure to be truly double-blind it would be necessary to "publish" the results regardless of the agreement or disagreement. This example demonstrates a difficulty in the use of such techniques in RSR since the results of the research program are not published in the usual sense of the word. However, we do strongly recommend that whenever experimental results are published as a verification of a code the author specify in detail the extent to which double-blind techniques were used. There are several examples of comparisons published in the open literature by vendors in which no such discussion was present. In fact, the implication of the articles is that there was post-experiment code adjustment. ("The code was not able to reproduce this event." Disagreements between theory and data were attributed to new phenomena.) [italics ours] There are obvious pitfalls here.

Those experimental code results used to demonstrate the safety of LWRs should be published in the open literature, where the procedures in both experimentation and analysis can be examined by the scientific and technical community. Such procedures are usually not.followed in engineering development; nor need they be. However, they are essential when the purpose of the research is verification of a hypothesis, especially when there may be vested interests in the outcome.

Double-blind procedures are not used at the lower levels of the experimental program, and some workers in the program have expressed the view that they are inappropriate at the separate effects level, or impossible at the LOFT level. Separate effects testing is considered to be engineering development rather than experimentation. They recognize that the code will not be ready in time to give realistic predictions of the LOFT experimental results, but that it would be unrealistic, and perhaps irresponsible, to lock up the results of the experiments until the code predictions are ready. We would agree that there is much that can be learned from an experiment such as LOFT even if there are not de- 
finitive predictions available, especially in terms of unexpected phenomena. A serious effort is needed and apparently now being made to instrument LOF $\mathrm{T}$ beyond the expected ranges of response, so that good data would be available for analysis even if LOF T behaved in an unexpected way. Yet some in the research program have expressed the view that the existence of many experiments (the LOFT sequence, Semiscale, and others) which must be explained by the same computer code would serve the same purpose as double-blind procedures. We disagree.

\section{Conservatism}

The present computer codes are incapable of simulating accurately the course of a LOCA in a commercial LWR. We remind the reader that the weaknesses of these codes arise from several sources: highly oversimplified physics equations (assumption of single-phase flow; inability to conserve momentum and energy); small number of nodal points; limited dimensionality (most codes assume one-dimensional flow); introduction of arbitrary adjustable parameters to allow for unknown response of the nodal points and system components (because the separate effects tests have not yet been done), and so on. However, RSR must respond to the needs of the AEC Office of Regulation (REG) and the needs of REG are immediate. This conflict between the state of the art of the codes, and the immediacy of the needs of REG is addressed by the creation of "conservative codes," also called "evaluation models" as opposed to "best estimate models." In a conservative code, all approximations and unknowns are fit into the code in such a way that the code should predict a worse accident than would actually occur. REG uses the conservative codes to help establish the safety of a reactor before it is licensed. If a commercial reactor is shown to be safe by a conservative code, then presumably it is safe in reality.

The important issue from the standpoint of safety is to what extent the "conservative" codes are truly conservative. The general procedure for establishing the conservatism of an assumption made in the code is to try other assumptions and to see the effect the various assumptions have on a critical safety parameter such as the "maximum cladding temperature."

The assumption that such procedures can lead to a truly conservative code may be the weakest link in the entire regulatory chain. To begin with, there is a fault in the logic alone. It does not follow that because an assumption is more conservative than another assumption, it is therefore conservative in an absolute sense. It is also not necessarily true that one can estimate the conservatism of an assumption by its effect on a single, albeit critical, parameter. For example, it was once thought that the assumption which led to the highest maximum cladding temperature was the most conservative. Later experience showed that a lower maximum cladding temperature reached at a later time (when the pressure differential across the cladding was greater) could lead to clad ballooning, and a more severe accident. There is an imbalance in the effort and detail taken in simulating reactor response, when compared to the way in which the "conservative assumptions" are chosen.
The computer codes are designed to recreate those interactions that the human mind might miss; the use of handwaving arguments to simplify these codes compromises the ability of the codes to find phenomena overlooked by the programmer.

Thus the predictive ability of codes is weakened when they are made "conservative." Since the "conservative codes" are considered "verified" even if the discrepancies in their predictions are $100 \%$ (but on the conservative side), it is dangerous to use them in a realm outside that in which they have been tested. A code which has made conservative predictions for the LOFT facility cannot be considered "verified," because it is not necessarily true that it will give conservative results for a large LWR.

Because of the inherent limitations of conservatism, we recommend that the assumptions used in these codes be examined in detail and that a justification in depth be made available to the broader technical community. We also recommend that realistic models play a greater role in the licensing procedure. RSR is, in fact, now emphasizing the development of realistic codes. We applaud this program and suggest that all possible support be given to the development of these codes and to the requisite separate effects and integral system tests.

\section{Outside review}

The AEC has recognized the contributions made by concerned citizens toward the safety of nuclear reactors, to the extent that it now regularly requests their participation in review meetings and has welcomed the creation of independent committees (such as this one) to study the safety program. RSR has recently created review groups to oversee some of the code and experiment planning and implementation, in order to stimulate new ideas, promote discussion, and better coordinate experiment and calculation. The Advisory Committee on Reactor Safeguards has often played the role of a review committee.

Despite this record, the amount of outside review in the research program has probably not been sufficient. We recommend that the small review groups which oversee the experimental and theoretical programs have greater participation by outside scientists and engineers, preferably those with no other connection to the nuclear reactor community, but with strong backgrounds in basic experimental and theoretical research. However, it is important that all outside review be done in such a way that it does not dilute the responsibility and accountability of the principal investigator in a particular project. And, as we have said repeatedly in preceding sections, we strongly recommend that all experiments and theories used to evaluate reactor safety be published in the open literature and where possible in refereed journals.

\section{J. Overall evaluation of the light-water reactor safety program}

In the preceding sections we have presented a detailed review of the present safety research program, mainly that of the AEC Division of Reactor Safety Research (RSR), which is intended to provide an improved foundation on which to base evaluations and regulatory operations. In this section we summarize our view of the safe- 
ty research program as a whole and address the questions of its overall quality, timeliness, integration, and resource balance.

Since its formulation in mid-1973, RSR has clearly made major strides in the formulation of a coherent LWR safety research program. In the experimental program, growing emphasis is being given to the study of separate effects intended to get an engineering understanding of the response of system components over wide ranges of conditions with applicability to both BWRs and PWRs. We strongly endorse this initiative. In carrying out the program, RSR is trying to involve scientists and engineers at universities and national laboratories much more broadly than in the past; again we endorse this trend and think that safety research can benefit from even more such involvement.

The quality of the separate effects tests seems reasonably high, although their scale and scope have been limited to date. Much more can and should be done (see Sec. VI.D), especially on heat transfer behavior, two-phase flow, perhaps even core blockage, as well as on phenomena that strongly influence reflood time and rate. We believe that it is essential to push separate effects tests on a broader basis at large scale to establish the upper range of our engineering knowledge. This calls for parameter ranges and sizes that are much closer to those typical of operating, as well as abnormal, reactor conditions. With regard to tests of nuclear fuel behavior, we are concerned about the slow progress in the Power Burst Facility (PBF) program and recommend a major push to make it successful, in order to get the necessary large-scale fuel data in the near future.

In trying to launch LOFT as its principal intergral systems test of LOCA behavior of ECCS concepts, RSR hopes to lay to rest much of the uncertainty that remains concerning PWR ECC effectiveness. Of course, LOFT at $55 \mathrm{MWt}$ differs in detail as well as scale from a typical $3300 \mathrm{MWt}$ PWR. (See Sec. VI.F for detailed considerations.) Our overall assessment is that LOFT cannot be regarded as a proof test of PWR ECCS behavior. Instead, LOFT represents a very important test bed to examine the behavior of the individual and coupled components during a range of LOCA conditions, as well as to examine our ability to calculate LOCA behavior with advanced codes. LOFT can be equally important, in our view, as a test bed to study the feasibility of possible alternate ECCS schemes for PWRs and to compare their effectiveness. However, as pointed out, the LOFT instrumentation needs to be upgraded in numbers, range, redundancy, and quality if we are to learn what we should from this important test series.

RSR hopes that advanced computer codes, now under development, will be able to predict the response of LOFT in some detail and that the LOFT series will thus "verify" the codes for PWRs. If advanced codes do, in fact, accurately predict LOFT behavior without excessive use of adjustable parameters, perhaps with the help of double-blind procedures to insure more independence of code calculations and experimental results, then one may achieve a better understanding of the extent to which "conservative" codes, developed for regulatory evaluation, are genuinely conservative in a well understood quantitative sense. Yet two very sobering aspects of our assessment must be emphasized.

First, one must face the fact that important results of the separate effects tests and of the LOFT series are years away. Moreover, the new series of advanced computer codes are only being started and will themselves require a period of years for maturation. We estimate that the cycle to accomplish a reasonably selfconsistent comparison between data and codes (namely, to obtain solid experimental results, develop the advanced codes, make detailed comparisons, repeat selected experiments, and/or alter codes in light of discrepancies) will require a minimum of several years, perhaps five to eight at the presently planned level of program resources, before the scientific and engineering community can reach consensus on its quantitative accuracy. More resources could probably shorten the period somewhat, especially in the more important separate effects tests previously mentioned, in more manpower and computer time to push code development, and in maintaining a flexible and responsive LOFT schedule. Nevertheless, a careful, convincing comparison will require time.

Secondly, having carried out this task, we will be in the position of making experiment/code comparisons only on integral system effects at a scale well below that typical of operational LWRs. For the PWR case, one may have more confidence if ECCS behavior goes as expected in LOFT, especially if the cycle of separate effects test/system test/computer code maturation is successful on a LOFT scale. Yet the scaling involves phenomena that are nonlinear and complex, and it is likely to involve engineering approximations that will be difficult to understand completely. Scaling compromises in LOFT, which have an influence that is hard to evaluate, may introduce additional uncertainty vis-a-vis full-scale PWR behavior. For the BWR case, the ECCS is thought to function with more certainty because of simpler primary system organization and absence of steam binding limitations. However, there is no BWR system-level experiment at the LOFT size, much less at full scale. Thus the case for BWRs rests almost completely on the degree of calculational ability, albeit for a somewhat simpler calculation, using the same general class of codes whose limitations we treated at length. (See Sec. VI.I.) Of course, matters can be improved for both PWRs and BWRs by study of important phenomena through large-scale separate effects tests; but three-dimensional effects, large nonlinear oscillations, major core blockage, and cross flow effects and the like are very difficult to address in that way.

Given the results from planned separate effects tests, LOFT data, and the advanced codes as now envisaged, our group is very skeptical about the extent to which one can expect to scale quantitatively our understanding of PWR LOCA and the transient system behavior to full size and conditions. We have similar, perhaps less firm, reservations about the BWR case. It is true that we are applying very high standards; however, we believe that the situation warrants such standards.

Thus, we conclude that the research program is at a critical juncture. We believe that the following avenues, 
which are not mutually exclusive, are possible for this program.

\section{Option A: Limit scope to the present program}

This option implies somewhat expanded and largerscale separate effects tests, PBF experiments, and LOFT series plus development of advanced codes, a situation that has been thoroughly discussed in our report. It is impossible to tell at present whether or not scaling by such a large factor (e.g., from LOFT to large PWRs) will prove sufficiently conclusive to provide a complete quantitative evaluation of the margin of conservatism in LWRs.

Within this alternative, the only substitute would be field experience which would have to be gained over an extensive time period with well-instrumented LWRs. The overall experience to date has been very encouraging with nuclear reactors of the LWR type. There have been no accidents with large consequences, and we note very intensive efforts in the AEC and in the nuclear industry to emphasize good engineering and sound procedures to maintain this record. However, a risk is hidden in the uncertainty of scaling our understanding of the present ECC concept and transient behavior. It is conceivable that one may lack convincing evidence of quantitative understanding at full scale to settle an issue to the satisfaction of the technical public, in the event that an incident of large possible consequences occurs during operation of the increasing number of LWR plants. Such a situation would be a very serious matter for the power industry and the public, since it might be difficult to refute strong pressures to operate plants at less than full rating. The U. S. power capacity could be placed in jeopardy by lack of definitive and demonstrable under standing.

\section{Option B: Augment the research program by pushing the investigation and development of alternative concepts to cope with LOCAs and with other transients of concern}

This means emphasis on concepts that are analyzable and scalable, or that can be shown conclusively to overwhelm the problems. We believe that there are very real possibilities here, especially for PWRs, suggested by members of the nuclear reactor community. These concepts which center on injection of fluid directly into the lower and/or upper plenum have not yet received a critical test. Testing such concepts is an important part of the LOFT series since they have features which may get around the technical uncertainties inherent in the present ECCS approaches.

It is not clear what such an approach, if successful technically, would do to reactor costs. In the short run, there might be pressure for retrofitting existing reactors and those under construction. In the long run, the stability to the nuclear industry, brought about by a convincing ECCS concept, would surely pay off. It is quite possible that costs associated with "analyzability as a design criterion" would be sufficiently offset by lower computing costs, faster licensing, more confidence in margin of safety, and the like.

It is likely that serious pursuit of alternate ECCS concepts would require additional resources for the RSR re- search program, but the costs would be amply justified if a successful new concept emerged. However, optimism here must be tempered with the realization that alternative concepts for ECCS have yet to be proven. This course of action requires a number of years for evaluation and has no guarantee of success. Therefore, Option B entails some of the risks associated with Option A.

\section{Option C: Augment the present program by larger inte- gral system tests combined with a move toward some standardization of $L W R$ designs}

The main thrust of this approach is to obviate basing the case so heavily on the scaling question. In its simplified form, one designs a sufficiently large integral system test program so that scaling is not in doubt. In reality, such larger-scale integral test results must be combined with results from separate effects tests and utilized with advanced codes in order that the integral tests have broad applicability. To have a convincing application of what is learned and, at the same time, a reasonable measure of control over design changes, some standardization of reactor designs would likely be necessary. By this we do not mean a single frozen reactor design, but rather a limited number of verified designs. New features would be introduced only after they were studied with advanced codes backed up where necessary with the large-scale tests.

This is a conservative approach similar in spirit to that pursued in aircraft system or weapon system programs. In the case of aircraft systems, for example, a design is based on careful calculation and scaled experimental studies. A full-scale system based on this design is then constructed and subjected to fairly exhaustive testing, which usually results in design changes. The process is iterated until a satisfactory first production series is undertaken, all members of which are basically alike. Test and evaluation continues with the result that modified production series of the aircraft which incorporate design improvements appear from time to time. One must not push the aircraft analogy too far, but it does have a number of instructive features: the problems of fully calculating and scaling the behavior of highperformance designs, the need to encourage and at the same time control design innovations, and the problem of ensuring adequate public safety.

What is especially pertinent here is the question of design maturation through large-scale testing (full-scale in the case of aircraft). For the LWR situation, there are surely non-nuclear as well as nuclear experiments which could be utilized at scales much closer to full scale than LOFT, and which can contribute to a more solid understanding of the safety of LWR technology.

As commented above, a likely price for this option is acceptance of some standardization of systems. Standardization itself raises many issues, some felicitous and some troublesome. It would certainly have benefits in terms of less complex quality assurance, more reliable maintenance, more effective inspection, better operator training, perhaps even lower overall costs. It would no doubt lead to more efficient and expeditious li- 
censing, as well as a better experience base that could make possible more confident and efficient operation within the margin of safety, with many benefits to power utilities. Of course, standardization has its possible penalties. Overstandardization could leave the program susceptible to an Achilles heel in the technology. Standardization may also slow the pace of change and innovation; if the bureaucratic procedures became too rigid, it could smother good ideas before they have a chance to be heard. Again, the aircraft industry provides an instructive example of how design improvements can be encouraged and at the same time controlled through a series of standardized production models. In any case, we are convinced that the spirit of innovation does not have to be stifled by the requirement that thorough largescale testing be used to test ideas before putting them into widespread practice.

Option D: Augment the research program by placing additional emphasis on better containment, consequence mitigation, and accident recovery

In addition to research intended to provide an under standing of LOCA behavior and ECCS effectiveness, we believe that considerable initiative should be directed toward better methods for containment, consequence mitigation, and accident recovery. On the side of improvement in containment, Sec. VI.G and VI.H pointed to a number of topics well deserving of attention. Among the more important of these are better methods to reduce pressure and remove heat inside the containment, and improved radioactive particle scrubbing technology. "Core catcher" concepts appear worth re-examination. Also, the concept of controlled filtered release (soft failure) seems not to have received serious consideration as a means of mitigating consequences of containment overpressure during an accident. We believe that it is important to put additional emphasis on careful investigation of such improvements, with judicious use of engineering hardware-level experiments. In addition, as discussed in Sec. VI.G and VI.H, we are disturbed by the lack of understanding of mechanisms of steam explosions. Experiments should be conducted to clarify this problem. Underground siting has been talked about (pro and con) a great deal, but we are not aware of a comprehensive study. Several of these topics are also very pertinent to the sabotage problem. Finally, phenomena important in radiological consequences and ground decontamination are among the least understood of all those pertinent to reactor safety. Much more needs to be learned (see Sec. VI.H), so that possible steps for better accident consequence mitigation and recovery can be analyzed.

To make progress in any of these areas in the present situation requires stimulation and coordination by a single responsible agency, clearly the AEC. However, we find that very limited AEC resources are directed to this end (see Sec. VI.B) and that the present system of incentives does not seem to stimulate much effort in the industry. We believe that an augmented research program in these areas is likely to be quite fruitful and is surely prudent.
Option E: Complement the present program through emphasis on remote and other conservative types of siting

Remote siting is often bandied about without much analysis as an "obvious" solution to all of our problems with power reactors. It is frequently countered, again often without much analysis, as impractical because of the costs of long-distance power transmission. We have not examined this type of alternative in any detail, but must report that we could not locate a comprehensive study of its pros and cons. The payoff in terms of increased public safety is not as simple as might be supposed. As we have observed in Sec. V, immediate deaths from high doses in the unlikely event of a large, uncontained accident are rather localized geographically. However, much of the expected total death, illness, property damage, and denial would come from total dose (man-rem) effects which are likely to be long-term and spread out over considerable distance (hundreds of miles). Therefore, it is important to evaluate the real safety advantage from more remote locations.

The economic analysis is also complicated by the need to take into account land, reactor system, and power transmission costs, along with relative costs of security measures, waste heat removal (or utilization), ecological impact, and the like. Safety, and possibly costs, may be favorably affected by grouping reactors in nuclear parks where mutual support among reactor auxiliary systems becomes possible and collective security measures are feasible for better control of sabotage.

We strongly recommend that remote siting options receive serious, careful study from the cost/risk/benefit point of view. We understand that an AEC-sponsored study of nuclear parks is already underway and may provide some of the needed perspective.

\section{Summary of options and resource allocation}

Full evaluation of any combination of these or other program alternatives really involves a cost/benefit $/ \mathrm{risk}$ analysis that is beyond the scope of anything we have done. As far as we have seen, it is beyond anything the $\mathrm{AEC}$ has done. What we can say is that (1) we are not convinced by the available experimental data and code calculations that a complete quantitative assessment of LOCA and transient behavior can be made at present; (2) we are skeptical that, given the results from the presently planned experiments and the advanced codes, the difficult scaling question can be unequivocally resolved. We view it as extremely important that means be found to bring about a convincing resolution of these issues.

Within the presently planned level of resources, the AEC's current program gives evidence of a shift toward limited investigation of parts of Option B (alternate ECCS), Option D (containment), and Option E (siting). If the budgets are to be restricted to this level, we would advise more emphasis on engineering hardware-level studies of Options B and D with funds diverted if necessary from Option A (present program), even though this would slow the main thrust of the present program in investigation of the current ECCS behavior. 
TABLE XXXI. Projected costs using lowest forecast of LWR capacity presented by the AEC.

\begin{tabular}{cccc}
\hline \hline Year & $\begin{array}{c}\text { Installed LWR } \\
\text { capacity (MWe) }\end{array}$ & $\begin{array}{c}\text { Cumulative capital costs, } \\
\text { (billions of \$) }\end{array}$ & $\begin{array}{c}\text { Annual cost of generated electricity } \\
\text { (billions } \$ / \mathrm{yr} \text {.) }\end{array}$ \\
\hline 1975 & 42000 & 25 & 2.8 \\
1980 & 84000 & 50 & 5.6 \\
1985 & 220000 & 130 & 14.5 \\
1990 & 380000 & 230 & 25.0 \\
\hline \hline
\end{tabular}

We believe that, in view of the urgency of the situation, the Safety Research Program is underfunded to do the job required of it. Assuming more resources are available, we recommend that Options B (alternate ECCS) and $D$ (containment) be fully implemented without slowing down investigation of the current ECCS concept. Furthermore, to provide an alternative to continuing dependence on ECCS design for which unequivocal conservatism may not be demonstrated, we recommend that immediate steps be taken to implement detailed analysis and planning for a large-scale integral system test program, Option C. If at the end of several years' study of alternative ECCS concepts, using LOFT tests and other studies, evidence cannot be seen for imminent success, the AEC will be in a better position to carry out the large-scale integral test program and will better understand its possible contribution.

To keep in perspective the costs of research and development programs associated with these options, it is helpful to estimate the present and prospective capital and operating costs associated with the LWR commercial electric power systems to which this research is relevant. To get a rough idea of these costs, we assume that the total (uninflated) capital costs of LWR power plants are $\$ 600 / \mathrm{kWe}$, that the costs of electricity are $10 \mathrm{mills} / \mathrm{kW}$ hour., and that the average load factor is $75 \%$. Using the lowest for ecast of installed LWR capacity presented by the AEC (AEC, WASH-1139, 1974), the resulting projected costs are presented in Table XXXI.

The total U. S. reactor safety program budget for FY1975 is about $\$ 100$ million, of which about half is accounted for by the AEC/RSR program, and the remainder by EPRI and vendors (assuming about $\$ 10$ million for the latter). This corresponds to about $3.5 \%$ of the present annual costs of electricity generated by LWR systems, and less than $1 \%$ of that projected for 1985 . Uncertainties in the capital and operating costs of nuclear power within the next decade are far greater than these precentages. It is clear that from an overall national standpoint substantial increases in the LWR safety research program budget in the next few years would not significantly increase total nuclear power costs.

Completely omitted from the above discussion is any reference to safety research on advanced types of nuclear systems. We have not conducted an extensive study of the safety problems of these systems, in view of the prior need for an extensive look at the LWR problems. However, we believe that it would be most unfortunate if these systems failed to receive sufficient attention in the near future to identify and understand early possible safety problems. The design and development of these advanced systems would certainly benefit, and the AEC could obtain more reliable cost/risk estimates for them. We believe it is particularly important to continue to push the experiments and advanced codes with the help of the best qualified scientific personnel in the country. Managing such an enterprise is no easy task. RSR has made laudable strides toward a program more open to scrutiny and to ideas, for example, by instituting review groups for RSR programs. More conscientious effort and interest within such organizations as the American Physical Society could be stimulated and utilized in an open program to good advantage. Specific means should be sought to bring this about.

$\mathrm{K}$. Organization and management of reactor safety research

Recent legislation which replaces the AEC by the Nuclear Regulatory Commission (NRC) and Energy Research and Development Administration (ERDA) sharpens the questions of the proper locus for reactor safety research and conditions for its successful management.

The obligations of the NRC can be listed briefly:

(1) Apply present regulatory system.

- review application and issue licenses

- apply acceptance criteria and inspect construction of new reactor installations

- conduct inspection, audit, and review of licensed reactor operations

- evaluate and respond to incidents, etc.

(2) Develop improved regulatory systems.

- take less time in the regulatory process for certain reactor designs

- modify its internal operation so that the NRC functions are not manpower limited

- work out new and better criteria for present design reactors (which could both increase assurance and reduce the necessity for de-rating reactors out of ignorance)

- develop criteria for new reactor designs

- evaluate possible alternative fuel and waste safeguarding requirements and procedures, etc.

(3) Serve both as an informed critic and customer of ERDA.

The above sketch recognizes the importance of the NRC and the high cost to our society of excessive delay and of unnecessarily stringent regulation on the one hand and of too loose regulation on the other. It recognizes also that reactors, reactor licensing criteria, inspection methods, and reactor siting opportunities are still evolv- 
ing and, further, that the NRC will have to develop quickly to be able to perform its role not only now and over the next few years but in the decades to come.

ERDA also has an important role to play with respect to the safety of nuclear reactors:

(1) Analyze alternative designs and safety questions relevant to new types of reactors it is considering for development.

(2) Explore new opportunities for reactor testing and for designs, including development of new computer codes, both for its own work and to provide NRC with improved capability for more realistic evaluation.

(3) Perform some of NRC's research, as well as technical development of inspection measures and computer codes, provided NRC decides to spend much of its reactor-safety-related funds with ERDA.

(4) See opportunities for improvement in performance and safety of existing reactors in view of its knowledge of existing reactors.

(5) Identify aspects of NRC's operation that need strengthening or changing as a result of ERDA's involvement in satisfying NRC's technical needs.

(6) Provide a perspective on alternatives to nuclear power which may be of broad utility in decisions of the power industry.

At one extreme, if all reactor safety research were lodged in ERDA, NRC might evolve into an automaton which, although initially effective, would be unable to improve its capabilities or to meet new opportunities and challenges as they arose. At the other extreme, if NRC had all the reactor safety responsibility, ERDA would be unable to guide responsibly its own investigations of future alternative reactor systems.

At the time of writing our report, the division of responsibility for reactor safety research between the two organizations is not yet clear. We offer the following observations based on our study. Considering the needs and the hazards, it seems preferable that most of the reactor safety research should be lodged with NRC, with sufficient money for staff, for development of codes and tests, for new techniques of inspection, for improving efficiency of operation, and for the occasional performance of a necessarily costly large-scale test to confront realistically the predictions of the analytical/com- puter techniques. To do its job, the NRC will also have to have a better idea of both the probability and the consequences of accidents involving reactors of both present and future design. It would also be natural and beneficial for NRC to spend a substantial portion of its reactor safety funds in the laboratories of ERDA. It is a matter for organizational invention in setting up this arrangement to see to it that NRC does much of its research and development in this way.

A division of responsibility that would honor these needs would be to assign to NRC primary safety research responsibility for those types of reactor moving into large-scale production (in the present case LWRs) and to ERDA primary safety research responsibility for those systems still in development, albeit in a different organization within ERDA than that actually carrying out the development, to avoid direct conflict of interest. Each should retain a secondary responsibility in areas of the other's primary responsibility in order that each be able to provide the necessary critique of the other's operations as well as to accommodate an orderly shift of responsibility when new types of systems develop and move into production. The needs of the public for information and independent evaluation could be served by review boards which could conduct reviews of the operations of both organizations at regular intervals with appropriate public documentation.

Regardless of how the matter is settled, it is imperative that the open program, now instituted, be maintained. If we are correct in our basic assessment of the complex LWR behavior (namely, that the ultimate goal of confident and unequivocal design evaluation must be founded on a much stronger code development program combined with a much improved experimental data base than presently exists, especially in system level tests), RSR has a difficult road ahead of it. NRC has an equally difficult task of utilizing the results of continuing safety research to adjust its licensing and inspection procedures to fit the evolving level of understanding and confidence. Issues of this sort are sure to arise with the passage of each new type of reactor concept from development into test, production, and operation. Only an open program can generate public confidence and fully utilize the many talents of the scientific and technical community in these tasks. 


\section{APPENDIX I: THE LOSS-OF-COOLANT ACCIDENT}

This appendix consists of several loosely knit sections generally related to the Loss-of-Coolant Accident (LOCA). The first two sections, I-A ("Generalized Description of Loss-ofCoolant Accident for PWRs and BWRs") and I-B ("Loss-ofCoolant Accident Without Effective Emergency Core Cooling"), have been reproduced wholly, or in part, from earlier definitive studies on the subject. Section I-C ("Relationship of LOCA Phenomena to the ECCS Acceptance Criteria") represents the results of a detailed review of the AEC's criteria for the evaluation of ECCS performance conducted by members of the Study Group during our Summer 1974 exercise at Los Alamos. This section presents a brief qualitative evaluation of the more important portions of the ECCS Acceptance Criteria as they are related to physical modeling of LOCA phenomena.

\section{A. Generalized description of loss-of-coolant accident for PWRs and BWRs}

The events and processes of an LOCA are developed to illustrate the system behavior and phenomena which must be accounted for by the calculational methods in order to prescribe the design and performance requirements for ECC systems.

\section{PWR-LOCA behavior}

Figure 15 depicts the generalized LOCA behavior for a postulated large break in one loop of a multiloop PWR primary coolant circuit. This characterization of the accident is derived from many calculations carried out for different pipe break locations for current PWR designs. The numbers on the figure are indexed to the following description of the course of the loss-of-coolant process.

Immediately following the pipe break, as the primary coolant is expelled from the rupture, the system experiences a rapid subcooled depressurization (1) causing the flow within the reactor core to accelerate for an outlet break (2) or decelerate for an inlet break (3). As the system depressurization contin-

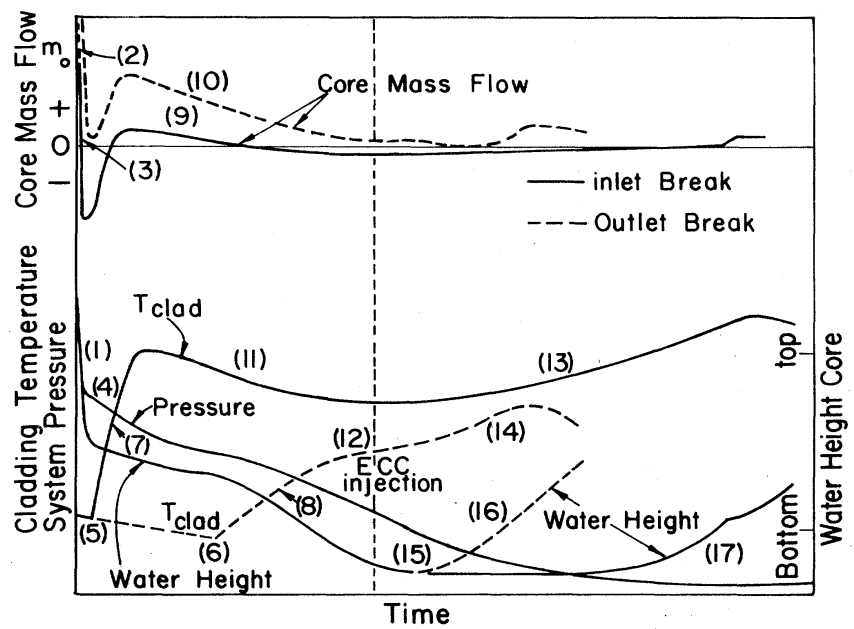

FIG. 15. Generalized loss-of-coolant behavior for large pipe breaks in a PWR.

\footnotetext{
${ }^{20}$ Reproduced, by permission of authors and American Nuclear Society, from Brockett, G. F., R. W. Griebe, R. W. Shumway, and J. O. Zane, "Loss of Coolant: Control of Consequences by Emergency Core Cooling," Proceedings of the International Conference on Nuclear Solutions to World Energy Problems, Joint A.NS/AIF Conference, November 13-17, 1972, Washington, D.C.
}

ues (4) the local fluid saturation pressure is reached and fluid flashing, with an attendant fluid density decrease, occurs in the core as steam bubble growth is initiated. Within the core region the decreasing fluid density (moderator loss) causes the core power generation to decline within a few hundred milliseconds to the fission product decay heat power level (approximately $6 \%$ of the operating power).

For the inlet break conditions the reduced core flow commensurate with coolant voiding in the core can cause a large abrupt decrease in heat transfer from the fuel to the coolant and initiate the critical heat flux (CHF), or departure from nucleate boiling (DNB) (5). For the outlet break condition the core flow increase (2) tends to offset the density decrease and high heat transfer is preserved for an extended period until the local fluid conditions within the core are degraded sufficiently that CHF (6) ultimately is reached. For either break, the abrupt decrease in heat transfer (5) (6) allows the large amount of stored energy within the fuel to redistribute with a resultant rapid increase in fuel cladding temperatures (7) (8).

For the inlet break condition, at several seconds into the depressurization process the core mass flow rate (9) is significantly reduced because of nearly balanced fluid resistance paths to the break. For the outlet break condition, the fluid resistance to the break from the core region is markedly lower and results in a continued significant upward core flow (10). These differences in the core flow histories (9) (10) respectively influence the cladding temperature histories for the inlet and outlet break conditions.

As the stored thermal energy within the fuel becomes redistributed the cladding temperature rise may terminate or the temperature may decline slightly (11) (12) as the competitive effects of continued fission product decay heating and some limited amount of heat transfer exist for a few seconds. As the coolant conditions within the core continue to deteriorate the cladding temperature rises (13) (14) commensurate with adiabatic conditions dictated by the local fission product decay heat rate.

When the cladding temperature exceeds $\sim 1200^{\circ} \mathrm{F}$ for either break condition, structural distortion, such as ballooning of the cladding, may develop. Ballooning is postulated to result from a combination of the decreased strength of the cladding (as the temperature increases) and the increasing differential pressure between the internal fuel rod pressure and the decreasing external system pressure.

As the coolant is expelled into the containment structure surrounding the reactor, the primary system continues to depressurize with an accompanying decrease of liquid level within the reactor vessel (15). When the system pressure decreases below the gas dome pressure within the ECC accumulators (or core flooding tanks), relatively cold auxiliary coolant is injected into the appropriate inlet piping (or upper core barrel region) in an attempt to replenish the liquid inventory in the bottom plenum of the reactor vessel.

For an outlet break condition, soon after accumulator injection begins, the liquid inventory in the bottom plenum is replenished to the bottom of the core (16). Core flooding is maintained by the low pressure coolant injection systems when the accumulator inventory is spent.

For an inlet break, some backflow from the core and continued boiloff of the liquid in the lower plenum cause steam flow up the downcomer which tends to inhibit the entry of auxiliary coolant to the lower plenum. In addition, the steam flow in the inlet pipes of the unbroken loops tends to entrain some of the injected coolant and this entrained coolant is then carried around the downcomer annulus to the break. These conditions lead to the postulated "accumulator ECC bypass" situation. As decompression continues and the system steam flow rates decrease, the influence of gravity overcomes the entrainment forces and the lower plenum begins to fill (17).

As the lower plenum fills and coolant reaches the bottom of the core, steam begins to be generated. The steam, entraining 
some liquid, rises in the core and cools the cladding. For the inlet break, the steam must escape from the system by passing through the steam generators and pumps in order to reach the system vent, or pipe break. The steam, in passing through the various system components and particularly the steam generator where additional energy is added from the secondary system, is impeded by friction. The frictional pressure drop can reach a value of several pounds per square inch causing a backpressure on the reflooding process which competes against the head of water in the downcomer attempting to drive coolant into the core. The downcomer head in most current reactor designs can develop to a maximum head of $\sim 7.5$ psi if the flooding process is relatively steady-state. If oscillatory effects occur as a result of the coupling between the inertance and the force of gravity on the liquid in the downcomer and the coupling between the inertance of the liquid in the downcomer and the compliance of the compressible steam volume above the flooding front, the average driving head could be less than the steady-state driving head, thus lowering still further the timeaveraged flooding rate within the core. The oscillatory flooding front could, however, provide heat transfer in the early part of the flooding process.

The competing effects of the limited driving head for flooding and the backpressure from the exiting steam give rise to the postulated steam binding problem. The limited flooding rate for the inlet break, resulting from steam binding, causes decreased heat transfer in the core relative to that which would exist for higher flooding rates for an outlet break. Where these competing effects are involved for the inlet break, additional subtleties, such as the effects of containment backpressure on entrainment and on heat transfer and such as the compressibleflow acceleration-pressure drops due to energy being transferred from the secondary side of the steam generator to escaping steam from the primary system, become important.

The temperature that the fuel cladding can attain without loss of structural integrity is determined, for zirconium-clad fuels, by the amount of oxygen taken up by the cladding during metalwater reactions which become significant at temperatures above $1800^{\circ} \mathrm{F}$. At $1800^{\circ} \mathrm{F}$ the reaction rate is low but as the temperature increases to $2000^{\circ} \mathrm{F}$ and above, the reaction rate increases rapidly. At $2300^{\circ} \mathrm{F}$, for example, the oxygen uptake is such that reaction durations exceeding several tens of seconds cause sufficient embrittlement that upon quenching of the fuel cladding by ECC, the structural integrity of the cladding is insufficient to assure a definable heat transfer geometry within the hotter regions of the core.

The foregoing, intended to depict the general system behavior during an LOCA for a PWR, has emphasized the DBA conditions which are expected to establish the ultimate requirements for ECC system design. The magnitude of the calculated effect of break size and location on the DBA is shown in Fig. 16. The calculations for developing the figure include those by the reactor manufacturers and those performed independently by Aerojet Nuclear Company in conjunction with the design and program planning for the Loss-of-Fluid Test (LOFT). Apparent from the figure is the dominant influence of the large inlet break in determining the requirements of ECC designs for PWRs. However, the very largest break should not be concluded to be the most demanding on ECC design for all PWRs.

\section{BWR-LOCA behavior}

Figure 17 depicts the generalized loss-of-coolant accident behavior for a postulated break in either the liquid recirculation lines or the steam outlet lines of a contemporary boiling water reactor system. The numbers on the figures are indexed to the following description of the course of the loss-of-coolant process.

Immediately following a steam line or recirculation line break of a BWR the system experiences a very limited subcooled de-
FIG. 16. Generalized comparison of maximum cladding temperature for various primary system pipe break conditions in a PWR.

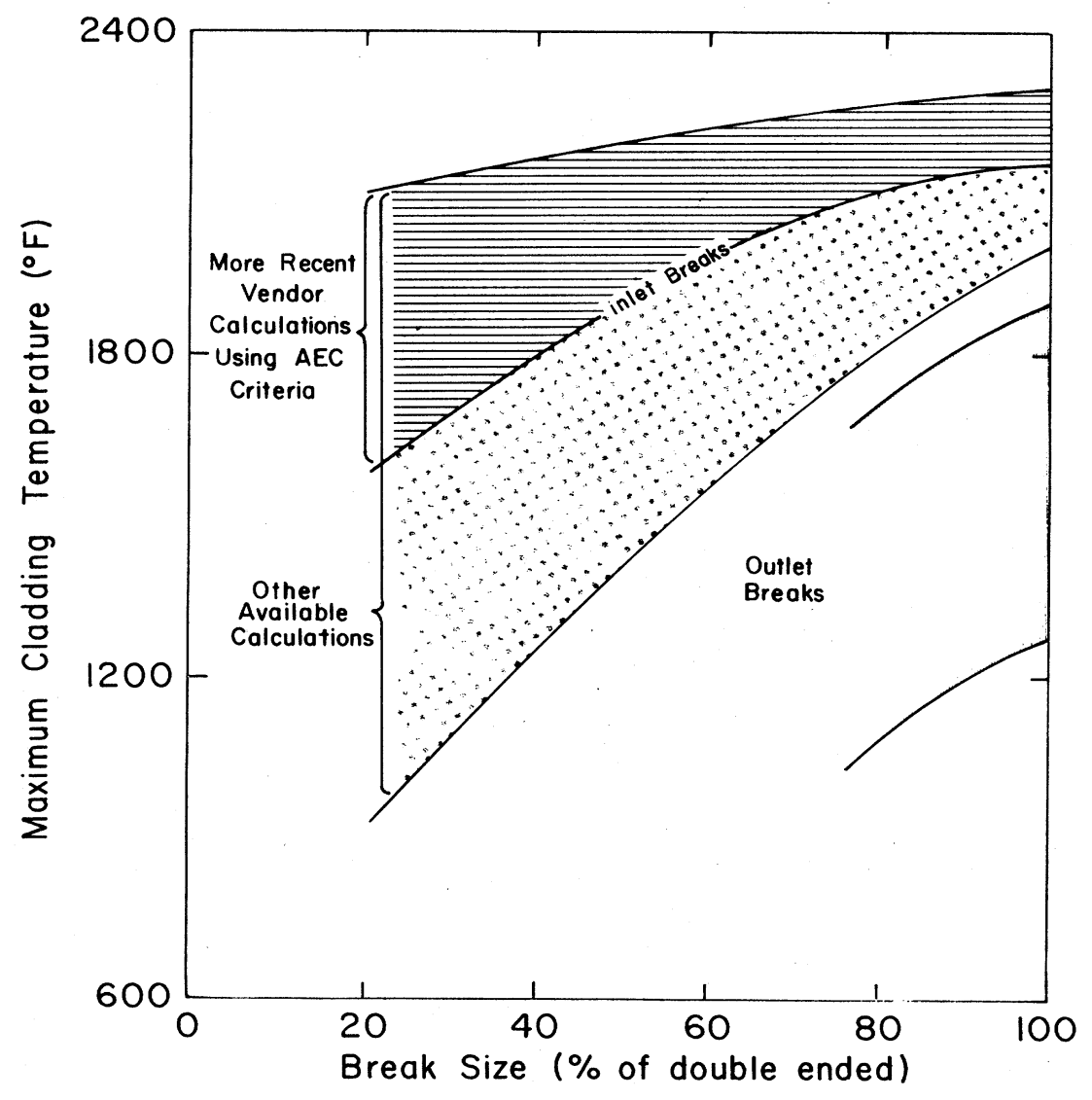




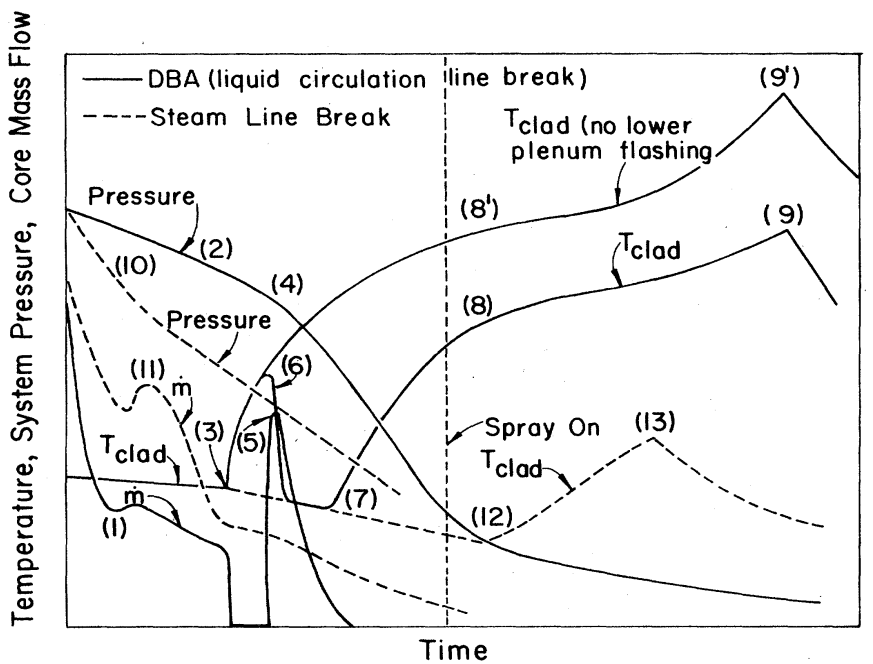

FIG. 17. Generalized loss-of-coolant behavior for large pipe breaks in a BWR.

pressurization because a significant amount of the fluid in the system during operation is at saturation conditions, with the remainder being slightly subcooled. The loss of one recirculating loop causes the core mass flow to drop rapidly to about one-half the initial value (1) as the other systems continue to provide coolant supply to the lower plenum, since a large volume of the vessel contains steam, at the outset, the depressurization process is relatively slow (2), and at several seconds into the transient, the steam isolation valves in the outlet line close requiring that all system coolant exit from the pipe break region. Since the contemporary version of the BWR incorporates the internal jet pump design, all pipe breaks, including recirculation and steam line breaks, in general, produce the effect of an outlet break in a PWR; that is, the depressurizing coolant flows in the normal upward direction through the core as illustrated in the figure.

At the approximate time the liquid level within the reactor drops to an elevation at which the jet pumps become uncovered, the mechanical pumps in the recirculation line are coasting down and shortly cavitate dropping the core mass flow to nearly zero. These conditions promote coolant starvation within the reactor core and initiate $\mathrm{CHF}$ in the hotter regions of the core (3). As the liquid level in the outer annulus around the core barrel drops to the elevation of the recirculation line outlet, the flow out the break becomes steam and the depressurization rate is increased (4). Simultaneously, the saturation pressure of fluid in the lower plenum of the reactor vessel is reached and a process called lower plenum flashing is initiated (5). During this process the fluid tends to flash violently and surges into the core region. The potential for significant cooling exists such that the cladding temperature rise may be terminated (6) and the cladding temperature may be restored to the fluid saturation temperature. As the coolant inventory in the lower plenum is spent from flashing, the system pressure continues to decline and the cladding temperature again rises in the hotter zones of the core and experiences DNB a second time (7). The cladding temperature rises rapidly until the energy redistribution within the fuel pin is complete at which time decay heat limits the rate of the temperature rise (8). Shown in the figure for completeness is the continued temperature rise from the early event of CHF (3) on through to the temperature limit $\left(9^{\prime}\right)$ assuming no cooling due to lower plenum flashing. As the system pressure continues to drop, a high pressure spray system above the reactor is initiated and top spray flow is developed at about 260 psia. The spray tends to wet the fuel canister walls providing a radiation sink for heat removal from the fuel pins. The resulting steam from canister wetting also provides some convective heat removal from the cladding surface. This cooling process tends to slow the heatup rate until the lower reactor vessel plenum is filled by the accumulated spray and LPCI system coolant inventories and a core reflooding process similar to that for the PWR is initiated.

For the lower cladding temperature history (8), the cladding temperature turnaround (9) results from the initiation of flooding at the bottom of the core. For the upper cladding temperature history $\left(8^{\prime}\right)$, the effects of metal-water reaction energy are seen to cause a significantly increased rate of temperature rise prior to the event of flooding $\left(9^{\prime}\right)$.

For the steam line break, the various events are depicted by dashed lines in Fig. 17. The pressure is seen to decrease considerably more rapidly (10) than for the recirculation line break. Since steam venting is taking place at a higher region of the reactor vessel the liquid fraction in the system remains high and all recirculation line systems continue to operate. Significant core flow is thus seen to continue (11); however, the flow eventually decreases as the pressure decay causes the recirculating mechanical pumps to cavitate. The flashing process continues to provide reasonable core flow and at least sufficient steam cooling to the core. The attendant cladding temperature indicates that nearly all the stored energy within the fuel is removed until, at the worst case, the coolant conditions can no longer support the heat transfer required to keep the cladding temperatures near the coolant saturation temperature (12). At this point the cladding temperature begins to rise as a result of the small amount of remaining stored energy and decay heat energy. Up to this time most of the fluid lost from the system as a result of a steam line break has been steam and some two-phase mixture; that fraction of liquid having insufficient enthalpy to flash remains in the lower plenum. The additional inventory necessary to fill the lower plenum to the bottom of the core and effect early turnover of the cladding temperature rise (13) is, therefore, considerably less than for the recirculation line break. The general behavior of the fuel cladding, effect of metal-water reaction, and embrittlement are sufficiently similar to those of a PWR that additional discussion is unwarranted.

Unlike the process for the PWR, the effect of steam binding does not appear to inhibit the rate of flooding because the steam need pass only through relatively small frictional pressure drop paths on its way to the break.

Figure 18 presents the calculated peak cladding temperature as a function of break area for steam line and recirculation line breaks for two separate single failure conditions in a contemporary BWR (General Electric Co., 1970). One case considers failure of the HPCS; the other considers failure of a diesel generator. These graphs are considered representative of a single-failure criterion approach to maximum cladding

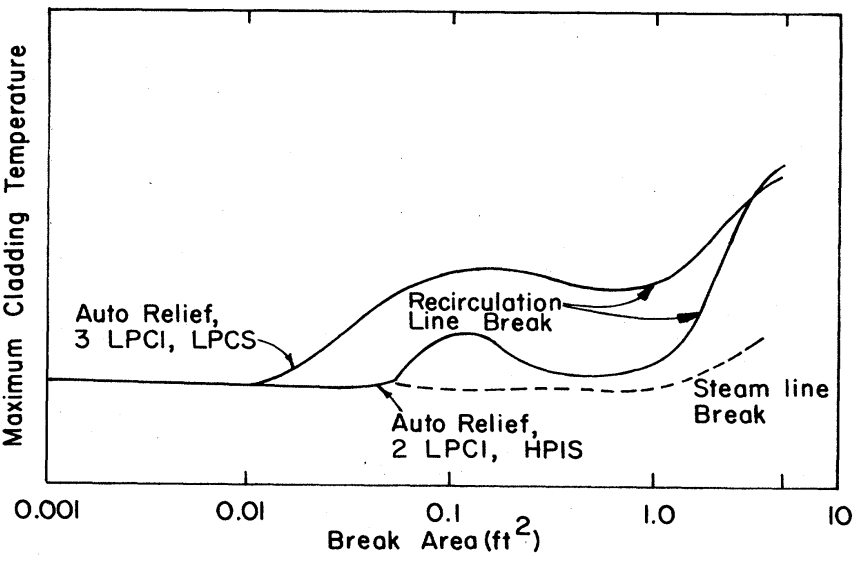

FIG. 18. Generalized comparison of maximum cladding temperature for various pipe break conditions in a BWR (from General Electric Co., 1970). 
temperature and should not be considered to be restrictive in defining the capability of a system or combination of systems. As would be expected, a general trend toward higher peak cladding temperatures occurs as break areas increase. For the smallest breaks, no core heatup occurs. The exact shapes and magnitudes of the temperature curves for this type of representation depend to a large extent on such factors as the analytical techniques used in the calculations, assumptions on heat transfer correlations, and the particular single failure condition considered.

\section{B. Loss-of-coolant-accident without effective emergency core cooling}

A probable sequence of events describing the core history during such a large LOCA without effective emergency core cooling can be divided into seven periods (adapted from AEC/ ECCS, 1967).

\section{Period 1: Blowdown}

Following the double-ended rupture of a large primary system pipe, there is a violent blowdown during which most of the water originally in the reactor pressure vessel is expelled into the containment. Three features of the blowdown influence subsequent core behavior. These are the blowdown forces, fuel element cooling during blowdown, and the rate of escape and the quantity of water remaining in the reactor pressure vessel after blowdown. Current estimates are that the blowdown forces will not seriously disrupt the core, that the fuel element cooling during blowdown could result in removal of about one-half of the stored energy in the fuel rods, and that about $10 \%$ of the water originally in the reactor vessel would remain after blowdown. At the completion of blowdown, which takes at least 5 to 10 seconds, for the largest breaks, core temperatures may range from about $600^{\circ} \mathrm{F}\left(320^{\circ} \mathrm{C}\right)$ to about $1500^{\circ} \mathrm{F}\left(820^{\circ} \mathrm{C}\right)$ at the hottest portion of the core. However, these estimates are fairly uncertain as we shall discuss below.

\section{Period 2: Core heatup-decay heating on/y}

Core temperatures begin to rise after blowdown, because of decay heat, at rates of approximately $20^{\circ} \mathrm{F} / \mathrm{sec}\left(11^{\circ} \mathrm{C} / \mathrm{sec}\right)$ for PWRs and $12^{\circ} \mathrm{F} / \mathrm{sec}\left(6.7^{\circ} \mathrm{C} / \mathrm{sec}\right)$ for BWRs at the hottest portion of the core. If they start from $1500^{\circ} \mathrm{F}\left(920^{\circ} \mathrm{C}\right)$, local core regions could reach temperatures greater than $2000^{\circ} \mathrm{F}\left(1100^{\circ} \mathrm{C}\right)$ within 30 to 50 seconds. Then the zircaloy-steam reaction begins to become a significant energy source. It is therefore important that the ECCS begin cooling the core before this critical juncture.

\section{Period 3: Core heatup-decay heating plus chemical heating}

As portions of the core reach temperatures greater than $2000^{\circ} \mathrm{F}\left(1100^{\circ} \mathrm{C}\right)$, the zircaloy-steam reaction begins to become significant. The rate of reaction initially increases parabolically with time. As cladding temperature increases, the reaction rate becomes dependent upon the availability of steam.

The time required to reach the melting point of the cladding, $3360^{\circ} \mathrm{F}\left(1850^{\circ} \mathrm{C}\right)$, at the hottest portion of the reactor in the absence of effective cooling, could be on the order of one minute. The beginning of localized clad melting may not mark the effective beginning of core collapse. Fuel rods may require temperatures greater than $3360^{\circ} \mathrm{F}\left(1850^{\circ} \mathrm{C}\right)$ before any clad dripping or fuel rod slumping can occur.

\section{Period 4: Core collapse and collection as a "Heap" in the reactor vesse/ without effective emergency core cooling}

The time from the pipe rupture to the collection of the core as a "heap" in the reactor vessel is very dependent upon the model chosen for core meltdown and the core structural grid plate failure. Core meltdown models range from melting and immediate dripping of zircaloy from the core directly into the bottom of the pressure vessel, to total holdup of a nearly fully molten core until such time as grid plate weakening or melting is achieved. The former model results in the gradual collection of the core in the lower pressure vessel head, while the latter model results in sudden dumping of a major portion of the core in a molten state into the lower pressure vessel head.

A time range from 10 minutes to 60 minutes from pipe break to collection of heap appears to cover the uncertainties. The lower limit, 10 minutes, derives from a heat accumulation consideration. With extensive metal-water reaction $(50 \%$ of cladding) and the accumulated decay heat, there is probably enough energy to bring the entire core on the average to the melting point of $\mathrm{UO}_{2}$.

The actual process of a core collapse and collection in the bottom of the reactor vessel is likely to be a very complicated matter, involving: (i) the formation of eutectics, (ii) dripping, molten flow, and refreezing, (iii) blockages of flow and steam for chemical reaction, and (iv) many other effects.

\section{Period 5: Pressure vessel melt-through}

The nature of a melt-through of the pressure vessel head is somewhat unknown. As noted in the above, it is not known whether the fuel would dribble through the grid plate slowly as the fuel melted, or if it would form a pool and melt through suddenly in a delayed action.

From $50 \%$ to $80 \%$ of the fuel might fall into the pressure vessel bottom. However, this part of the fuel would include that portion of the core having the greatest decay heating.

The first effect of the core material's falling into the vessel bottom would be to evaporate rapidly any water in the vessel. A rapid production of steam might affect the flow of core material through the grid plate and might blow molten core material up, through, and out of the pressure vessel.

Radioactive decay and possibly some further metal-water reaction would result in continued heating of the fuel and pressure vessel bottom.

The weight of core material of about 250 tons would cause forces in the vessel of only a few percent of those due to normal operating pressure, hence the vessel bottom would not be apt to fail until its melting point were approached.

A melt-through of the pressure vessel could be expected to occur in from 20 to 60 minutes after core collapse (30 to 120 minutes after pipe rupture), if there is no extra cooling.

\section{Period 6: Pressure vessel melt-through to collection as a heat at bottom of containment}

Pressure vessel melt-through might be expected in 30 to 120 minutes after the pipe rupture. It seems certain that meltthrough will be a catastrophic event, in that large quantities of molten material will be discharged suddenly. Most of the core, in addition to a melted portion of vessel bottom, would come through very quickly. A major question concerns the interaction of the molten stream with the water pool likely to be at the bottom of the containment vessel.

The molten core material, in falling into a water pool, may cause steam explosions. Our predictive ability of steam explosions is very limited. About all that can be stated is that localized explosions yielding local pressures of the order of 3000 psi (200 atm) might occur.

The direct damaging effects of localized explosions are probably rather minor because of the size and strength of the struc- 
tures in the lower regions of containment vessels. The most serious effect of such explosions might be the tendency to scatter molten core materials to high regions of the containment wall where localized melt-through might occur.

Another serious effect of localized explosion is to promote subdivision of the molten core material and increase the overall rate of the reaction of water with the metallic constituents of the molten core material. The increased production of hydrogen is the serious feature which leads to containment failure by gradual hydrogen overpressure in spite of the successful operation of containment sprays or suppression pools.

\section{Period 7: Behavior of molten core mass at the bottom of the containment}

On failure of the pressure vessel bottom, it is estimated that as much as 45 to $75 \%$ of the core would fall into the containment vessel and that the self-heating of this material for a total elapsed time of from 1 to 2 hours would be of the order of $0.5 \%$ to $0.6 \%$ of the reactor power or 15 to $18 \mathrm{Mw}$ ( $50 \%$ of the total decay heating).

The core material, together with the molten vessel material, grid plate, etc., would weigh about 100 to 200 tons. The molten core material would displace any water into which it fell and commence to heat the concrete and other materials between it and the containment vessel. The rapid boiling of any water into which the molten core fell might scatter the molten core. The nature of the molten core and its flow behavior are unknown. Whether it would rapidly spread out into a thin layer or remain more in a heap is uncertain.

The concrete would spall and disintegrate as it was heated by the molten core. Since the molten fuel-steel mix is heavier than the concrete ingredients, the concrete residue would either mix with the core or float on the top.

Ordinary dried concrete has a density of about 145 to $155 \mathrm{lbs} /$ $\mathrm{ft}^{3}$ (specific gravity of 2.32 to 2.48 ) and contains about $2 \%$ by weight of incorporated water, or about $20 \mathrm{lbs}$ of water per 100 lbs of Portland cement used. Its thermal conductivity is about 1.2 to $2.0 \mathrm{Btu} / \mathrm{hr}$ ft ${ }^{\circ} \mathrm{F}$ or 0.005 to $0.008 \mathrm{cal} / \mathrm{sec} \mathrm{cm}{ }^{\circ} \mathrm{C}$ and its specific heat is about 0.20 to $0.24 \mathrm{Btu} / \mathrm{lb}{ }^{\circ} \mathrm{F}$ or cal $/ \mathrm{g}{ }^{\circ} \mathrm{C}$. It virtually disintegrates at about $932^{\circ} \mathrm{F}\left(500^{\circ} \mathrm{C}\right)$.

If there is an average thickness of 5 feet of concrete between the pressure vessel and the bottom of the containment vessel, the disintegration of from 40 to 400 tons of concrete might be required for the molten fuel to reach the containment vessel.

It is estimated that the molten core might reach and penetrate the containment vessel in from a few hours to a few days after pipe rupture.

If there is no water at the lower part of the containment, or if the water is boiled away from that region and condensed elsewhere, then the dry mass would be expected to vaporize into higher areas of the containment at a very significant rate. Recondensation of vaporized fuel and fission products would occur throughout the volume of the containment.

\section{Relationship of LOCA phenomena to the ECCS acceptance criteria}

The critical LOCA phenomena are displayed in Table XXXII in direct relationship to the corresponding elements of the AEC's Acceptance Criteria (AC) (See Appendix III). The material presented in Table XXXII is divided into phenomena occurring during three time periods: Blowdown, Refill, and Reflood. The relative significance of each phenomenon has been identified in the Table in a qualitative fashion (as being of firstorder importance, $\mathrm{O}(1)$; or $\mathrm{O}(2)$, second-order importance). Qualitative evaluations of the conservatism of the elements of the AC presented are also given in the Table along with brief comments qualifying the estimates of conservatism. A supplemental discussion of the material presented in the Table is given below.

\section{Blowdown phenomena}

The energy sources, most of which act over the entire LOCA to a varying extent, are listed in the blowdown period. Those sources which represent basic energy inputs to the problem, the initial stored fuel energy (which depends upon the steady-state operating conditions of the reactor immediately preceding the accident), the fission heat (associated with shutdown reactivities and reactor kinetics), the decay heat of the neptunium, plutonium, and other heavier actinides, and the fission product decay, are all required to be calculated in a relatively conservative fashion in the AC. In the case of the initial stored fuel energy, although the AC specification is aimed at maximizing estimates of this parameter, the data base supporting the time and temperature dependence of the $\mathrm{UO}_{2}$ conductivity and gap conductance is weak. Moreover, since the AC do not prescribe conservative estimates of the parameters influencing the initial stored energy, the conservatism of this criterion in practice may not be very substantial. In practice, application of the initial stored energy criterion might produce values only slightly greater than "best estimates" of this aspect of the energy to be dissipated during the LOCA.

In the case of the fission product decay, examination of the best current literature in this field [e.g., Shure (1972), and Perry et al. (1973)], as well as discussions with prominent workers in the field, has led us to conclude that the AC prescription of utilizing the proposed ANS standard for fission product decay heat, ANS 5.1, plus an additional $20 \%$, will produce an estimate of decay heat which is conservatively high by about one standard deviation from mean estimates. Whether this conservatism, associated with one of the most significant energy sources, is adequate or not depends upon one's point of viewand was a subject of some debate within the review group. However, irrespective of the conservatism of the proposed ANS 5.1 standard, taken as an individual separate effect, it is probable that the overall AC specifications for plant operating characteristics are sufficiently conservative (cf., footnote, Table XXXII), as initial conditions for the LOCA calculations to assure that predictions for the net effect of these input energy sources will also be conservative. Thus, taken as a whole, the input energy sources appear to be generally conservatively prescribed within the AC.

Other energy sources entering into the problem are a function of the thermal history of the LOCA itself. Of these sources, the reactor internals heat transfer and PWR primary-to-secondary heat transfer prescriptions appear to be adequately prescribed within the AC. That is, following the AC prescription would lead to energy estimates for these sources which are nearer "most probable" or "best estimate" than particularly conservative values. However, it should be observed that the metal-water reaction rate prescription, for the high-temperature reaction of zirconium alloy cladding with steam, is very conservative with respect to energy release estimates. The AC requirement for use of the Baker-Just (B-J) equation for oxidation estimates can be shown to provide estimates which approach the upper limits of empirical observations for oxidation in the metal-water reaction. (cf. Scatena, 1972)

As a general observation, the LOCA derived energy sources are reasonably, or strongly, conservatively estimated. However, the $\mathrm{AC}$ have excluded from consideration a heat source of potentially overwhelming importance to PWRs-ruptured steam generator tubes. Based upon evidence of substantial tube corrosion problems with frequent associated leakage in most PWRs (Babcock \& Wilcox reactors seem to be exceptional cases, with few or no corrosion problems), it was the consensus of the group that steam generator tube failure during a severe LOCA could occur frequently. Moreover, it appears that rupture of a few tubes (on the order of one to ten) dumping secondary steam into the depressurized primary side of the reactor system could exacerbate steam binding problems and induce essentially uncoolable conditions in the course of a LOCA, for PWRs with ECCS of current design. 
TABLE XXXII. Critical LOCA phenomenological behavior and related ECCS criteria.

\begin{tabular}{|c|c|c|c|}
\hline LOCA phenomena & Related criteria (source) & $\begin{array}{l}\text { Influence } \\
\text { coefficient }\end{array}$ & $\begin{array}{l}\text { Criteria conservatism } \\
\text { and commentary }\end{array}$ \\
\hline
\end{tabular}

I. Blowdown period

A. Energy sources (input

Parameters

1. Initial stored fuel energy (steady statebefore accident)

2. Fission heat (shutdown reactivities and reactor kinetics)

3. Decay of actinides ( $\mathrm{Np}, \mathrm{Pu}$, U-isotope decay)

4. Fission product decay

B. Energy sources (LOCA derived)

1. Reactor internals heat transfer (piping, vessel walls, nonfuel internal hardware)

2. PWR primary-to-secondary heat transfer (steam generator/primary fluid)

3. Metal-water reactions (rate of energy release, $\mathrm{H}_{2}$ generation, clad oxidation)

C. Structural behavior

1. Pipe whip

2. Core loads

3. Steam generator loads/

tube failures

D. Primary system coolant behavior

1. Break flow

a. Break characteristic spectrum

b. Discharge model

2. Break location (determines system flow stagnation points)

3. Frictional $\Delta p$ (losses in pipes, core, other components)

4. Conservation of momentum equations in codes

5. Pump $\Delta p$

6. ECC fluid "bypass" of core

7. Core flow distribution (Blockage and radial flow)
Appendix K-Sec. IA

(IA1) ${ }^{b}$ Use case of highest stored energy considering: $\mathrm{UO}_{2}$ conductivity, gap conductance, fuel densification, gases within fuel rod, clad creep, etc. (IA2)

Actinide decay heat for fuel cycle time yielding highest calculated fuel temps (IA3) ANS $5.1+20 \%$ (IA4)

First order $\mathrm{O}(1)$

Strong (S)

(1) - BWR

$\mathrm{O}(2)$ - PWR

Second order

$O(1)$

$\mathrm{O}(1)$

Conservative by one $\sigma$ above mean

Heat transfer shall be accounted for (IA6)

Second order $\mathrm{O}(2)$

Adequate (OK)

Shall be accounted for (IA7)

(IA5) Use B-J equation; no steam limiting; if clad rupture, consider inside clad surfaces also

Not treated (NT) (see $10 \mathrm{CFR}$ 50-Appendix A)

NT

NT

(IC1)

Double ended/longitudinal splits-up to largest pipes. Moody-after $2 \phi$ attained.

Vendor must demonstrate highest temp. achieved.

§50.46-10 CFR 50

(IC2) Use "realistic" friction factors and $2 \phi$ friction multipliers (permitted models

specified)

(IC3) Neglect of elements or effects shall be justified.

(IC6) Pump model requirements specified. $2 \phi$ flow data required.

(IC1) "End-of-bypass" definition. Fluid injected before end-ofbypass must be subtracted from reactor vessel inventory.

(IC7-PWR's) Flow calcs. must account for cross-flow induced by fuel rod swelling blockage.
$\mathrm{O}(1)$

$\mathrm{O}(2)$

$\mathrm{O}(1)$

$\mathrm{O}(1)$

$\mathrm{NT}-(\mathrm{NC}) \mathrm{c}$ $\mathrm{NT}-(\mathrm{NC})$

$\mathrm{O}(1)$

OK-but steam generator tube leaks neglected)

S (B-J approaches upper limit for oxidation)

$O(1)$

S

$\mathrm{O}(1)$

OK

OK

OK-but limited data available

OK-but weak data base for "end-ofbypass" evaluation.
2. Homogeneous

flow $-\mathrm{S}$

3. $\mathrm{UVUT}^{\mathrm{c}}-\mathrm{NT}($ (?) 
TABLE XXXII. (Continued)

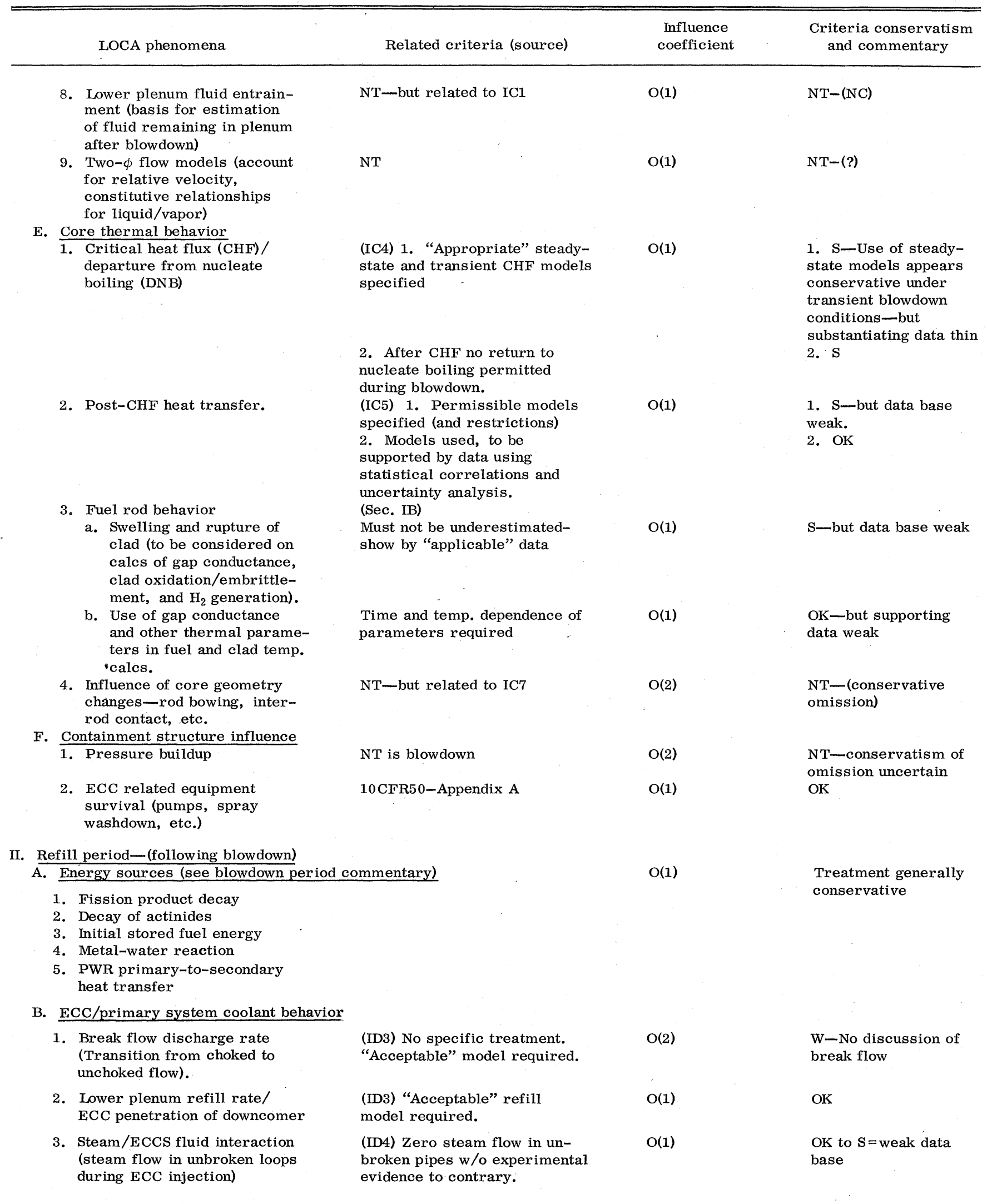


TABLE XXXI. (Continued)

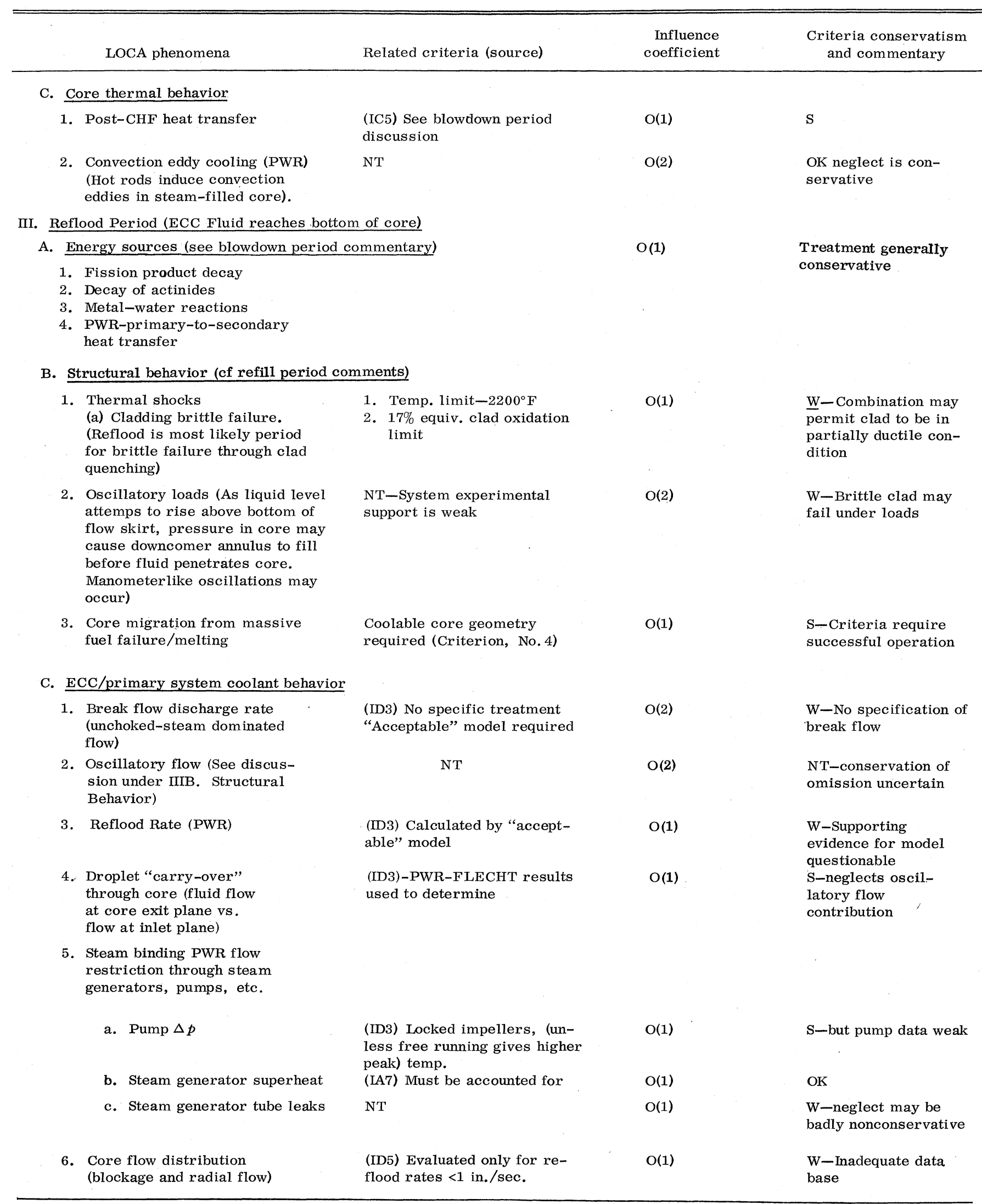


TABLE XXXII. (Continued)

\begin{tabular}{|c|c|c|c|}
\hline LOCA phenomena & Related criteria (source) & $\begin{array}{r}\text { Influence } \\
\text { coefficient }\end{array}$ & $\begin{array}{l}\text { Criteria conservatism } \\
\text { and commentary }\end{array}$ \\
\hline \multicolumn{4}{|l|}{ D. Core thermal behavior } \\
\hline 1. PWR reflood heat transfer & $\begin{array}{l}\text { (ID5-PWR) Based upon } \\
\text { FLECHT results }\end{array}$ & $\mathrm{O}(1)$ & OK \\
\hline a. Oscillatory flow (PWR) & $\begin{array}{l}\text { (ID5 and commission } \\
\text { opinion) } \\
\text { (Oscillatory flow benefits } \\
\text { disallowed) }\end{array}$ & $\mathrm{O}(2)$ & $\begin{array}{l}\text { S-Inclusion might } \\
\text { improve heat transfer }\end{array}$ \\
\hline $\begin{array}{l}\text { b. PWR low reflood rate } \\
\text { restrictions }\end{array}$ & $\begin{array}{l}\text { (ID5) Rates }<1 \mathrm{in.} / \mathrm{sec} \text {, heat } \\
\text { transfer by steam only, flow } \\
\text { blockage accounted for }\end{array}$ & $\mathrm{O}(1)$ & $\begin{array}{l}\text { OK-but data base } \\
\text { weak }\end{array}$ \\
\hline $\begin{array}{l}\text { c. Containment pressure } \\
\text { influence }\end{array}$ & $\begin{array}{l}\text { (ID2) Lowest calculable pres- } \\
\text { sure to be used for evaluation } \\
\text { of heat transfer coefficients } \\
\text { (PWR only) }\end{array}$ & $\mathrm{O}(2)$ & $\begin{array}{l}\text { S-but data base is } \\
\text { weak. (Increased } \\
\text { pressure improves } \\
\text { heat transfer.) }\end{array}$ \\
\hline $\begin{array}{l}\text { 2. BWR core spray/reflood heat } \\
\text { transfer }\end{array}$ & $\begin{array}{l}(\mathrm{ID} 6,7) \text { Based upon FLECHT } \\
\text { results }\end{array}$ & $\mathrm{O}(1)$ & OK \\
\hline $\begin{array}{l}\text { 3. Core migration and pressure } \\
\text { vessel melt-through }\end{array}$ & $\begin{array}{l}\text { Coolable geometry required } \\
\text { (Criterion No. } 4 \text { ) }\end{array}$ & $\mathrm{O}(1)$ & $\begin{array}{l}\text { NT-Criteria requires } \\
\text { successful ECCS } \\
\text { operation }\end{array}$ \\
\hline
\end{tabular}

a Energy input terms must assume: continuous reactor operation at 1.02 times licensed power level; max peaking factor allowed by tech. specs. Range of power distribution shapes and peaking factors which could occur over core lifetime must be investigated and the one used must result in "most severe calculated consequences" for spectrum of pipe breaks and single failures analyzed.

${ }^{b}$ Numbers in parentheses, e.g. (IA1) refer to specific numbered sections of the Acceptance Criteria.

${ }^{\mathrm{c}}$ Not treated in AC, (NT); Nonconservative omission, (NC); Unequal Velocities, Unequal Temperatures (UVUT) in multiphase fluid flow.

The specifications of criteria pertaining to structural behavior in the LOCA is not treated within the AC. Some of the reactor equipment, especially those elements external to the nuclear supply system (pipes, pumps, equipment within the containment building such as spray, cooling, and washdown piping etc.) are covered by other portions of the reactor criteria (e.g., $10 \mathrm{CFR}$ 50-Appendix A). But internal elements of the nuclear steam supply (the core, steam generator, and tubes) apparently are not covered within any of the specifications. Although structural behavior is not overlooked in reactor design and appears to be generally well engineered, the omission of structural loading criteria for the critical internal elements of the nuclear steam supply system seems like poor engineering, if not a nonconservative omission.

During blowdown, the AC specification of elements of the hydrodynamics of the primary system coolant behavior are generally adequate. The requirement to analyze the spectrum of potential break sizes within the nuclear steam system for double-ended breaks and longitudinal splits of any pipe in the system in determining the most severe LOCA conditions is characteristic of those phenomena which have been treated with strong conservatism. However, there are some potentially troublesome weaknesses which may more than counterbalance the strong points. In modeling the break flow, the AC prescribes the use of the Moody correlation (AEC Commissioners, 1974, p. 1112). The Moody method was developed upon an idealized model of isentropic, equilibrium flow in a pipe. As such it models homogeneous, equilibrium, two-phase flow in an apparently conservative fashion. It does not account for metastable flow of subcooled liquids which occurs at the immediate outset of flow from the break. Though such flows are probably of short duration, they are associated with flow rates which are generally over a factor of two greater than equilibrium flow. The Commission acknowledged in its Opinion on the AC (AEC Commissioners, 1974, p. 1112) that such transient effects may have an important effect on peak cladding temperatures. An ANC transient calculation was reported to have predicted peak temperatures about $100^{\circ} \mathrm{F}$ greater than a calculation using a fixed Moody discharge coefficient, as specified in the AC. In addi-in tion, the Moody model does not describe conditions where unequal velocities and temperatures may exist between liquid and vapor within the two-phase mixture. The effect of mistreatment of conditions of flow of this type is uncertain, as no calculations of system response with an adequate model of these conditions are known to have been made. However, in general, it appears that exclusive use of the Moody model may not be conservative.

that exclusive use of the Moody model may not be conservative.

In estimating core flow distribution under conditions where blockage may induce radial flow within the core, it has not been demonstrated that the current one-dimensional analysis methods used by the AEC and vendors are capable of conservatively predicting these effects. In very preliminary calculations, Gambill (1973) has shown that even limited local blockages - as well as substantial ones (from $20 \%$ to $90 \%$ ) -within a variety of overall core blockage conditions (especially from $0 \%$ to $70 \%$ ) can induce increases in the peak cladding temperatures of from hundreds to thousands of degrees F. Potential code weaknesses in properly modeling flow under these conditions may make estimates of overall ECCS performance conservatism suspect.

The AC do not specifically treat the problem of entrainment of fluid in the lower plenum during blowdown. The quantity of fluid remaining in the lower plenum, is directly related to the time interval (the refill period) between the end of blowdown and the beginning of reflood, during which very limited cooling of the fuel rods can be developed. Under these limited cooling conditions, temperatures would increase rapidly (perhaps as much as $20^{\circ}-40^{\circ} \mathrm{F}$ per second). The lack of specific AC treatment of this problem tends to be nonconservative. However, the requirement of the AC, Section IC.1, that all ECC fluid in- 
jected into the reactor vessel or inlet lines during the bypass period be subtracted from the calculated reactor vessel inventory, may be so strong that little or no fluid may be calculated to remain in the lower plenum at the end of the bypass. If this is true, then the question of lack of AC treatment of the entrainment of fluid in the lower plenum may be moot, so far as the conservatism of the lower plenum fluid entrainment model is concerned.

The AC also do not attempt the evaluation of nonhomogeneous two-phase flow models - in which there could be differences in relative velocity between liquid and vapor-nor provide for relationships between the constituents of heterogeneous mixtures. Without doing a numerical analysis of such models it is not possible to evaluate whether this AC omission is conservative or nonconservative.

In specifying the criteria to apply to core thermal behavior during blowdown, a serious attempt has been made to require conservative treatment of these phenomena. In particular, the specification precluding a return to nucleate boiling throughout the blowdown period after critical heat-flux (CHF) first occurs represents a strong conservatism.

Also the specification that the swelling and rupture of cladding during the LOCA must not be underestimated (AC, Sec. IB) implies strong conservatism in calculations of gap conductance, cladding oxidation, and embrittlement, and hydrogen generation. The same section requires that these parameters shall be utilized in estimating fuel and cladding temperature, accounting for such effects as swelling and rupture. In performing calculations of the temperatures of the hottest rods in the core, such requirements should be strongly conservative-especially if axial heat conduction along the fuel rod is neglected in the calculational method (as it is in many codes).

The unfortunate aspect of most of the methods specified by the $\mathrm{AC}$ for treating core thermal behavior during the blowdown is that the experimental data base supporting most of the semiempirical models is weak. As a consequence, though the criteria have been directed toward achieving assured conservatism, the empirical basis for demonstrating the conservatism appears to be weak.

\section{Refill period phenomena}

During the refill period, the dominant source of energy in the system is that due to radioactive decay of fission products and actinides. By this stage of the LOCA, it is probable that the initial stored energy would have been largely transferred away from the fuel and cladding with the expelled system coolant, so that it would no longer be a major energy source. Metal-water reactions, however, become an increasingly important source of energy as cladding temperatures increase. As a result of very low coolant flow rates, especially in the unbroken legs of the system, transfer of heat from the secondary to the primary side of the system will also be a relatively minor contribution to input energy during this period-assuming there are no steam generator tube leaks. As was discussed in connection with the description of these energy sources in the blowdown period, their AC specification appears to be generally conservative.

During the refill period, the principal hydrodynamic effects are associated with refilling the lower plenum and the effects of ECCS fluid plugging of steam flow in the unbroken steam generator loops of the system. The criteria in both of these instances are adequate or strong.

The core thermal behavior during this period is dominated by heat transfer to slowly moving saturated or slightly superheated steam. The AC specify the use of allowable post-critical-heatflux $(\mathrm{CHF})$ heat transfer correlations-as discussed under the blowdown period section. These models appear to be at least adequately conservative-and perhaps strongly conservativeespecially when utilized in PWR codes which do not consider inter-rod/coolant radiant heat transfer. It is also probable that the thermal gradients existing within a PWR core would induce convection eddy-currents which would aid the cooling of the hot central rods. This aspect of the core thermal behavior has not been treated in the AC. Its omission is apparently conservative though probably of second order in importance.

\section{Reflood period phenomena}

The energy input sources during reflood are generally the same as in previous periods. As in the previous periods, the AC treatment of energy sources remains generally conservative during the reflood period. The relative importance of the sources is somewhat different during this period, however. If cladding temperatures are relatively low, the dominant energy source is the decay heat. The influence of initial stored energy would be essentially negligible in this period. However, metalwater reactions can become important during reflood, since the highest cladding temperatures are generally expected to occur during this period. If temperatures exceed about $2000^{\circ} \mathrm{F}$, metal-water reactions would become a significant source of energy and would provide a positive feedback mechanism which would be hard to control: high temperature would induce the steam-metal reaction which would in turn cause even higher temperatures. This would be difficult to control without adequate reflood rates.

Heat transfer from the secondary steam forming loop of the system to the primary reactor loop becomes a very important energy source of PWRs during reflood. Fluid "carryover" through the core during reflood passes through the steam generators where the hot tubes (from the secondary fluid) dry and superheat the steam. The increased volume of heated steam produced in the steam generators of the unbroken loops would then attempt to displace the steam in the reactor core, in order to escape through the break. The resistance to this steam flow path provided by the fluid reflooding the core, as well as frictional effects in the unbroken steam generator loops, results in an increase in pressure. This pressure increase in the system is referred to as steam binding-a process which restricts flow through the core, potentially reducing the reflood rate. Steam binding apparently may cause hazardously low reflood rates, reducing them from intended values of 6 to $11 \mathrm{in} / \mathrm{sec}$ to rates of from 1 to $2.5 \mathrm{in} / \mathrm{sec}$ (AEC Commissioners, 1974, p. 1092). The influence of such low reflood rates on core heat transfer will be discussed below.

Structural behavior is especially important during the reflood period. The reflooding process has the potential for producing substantial thermal shocks to the fuel rod cladding, as quenching occurs. If metal-water oxidation has been excessive, brittle failure of the rods may occur during quench-or when they are subject to other loading mechanisms after they have cooled further. If the fuel rods are maintained at temperatures near the AC limits of $2200^{\circ} \mathrm{F}$ for periods on the order of two minutes or more, the oxidation produced by the metal-water reaction may leave the rods in a sufficiently embrittled condition so that quenching and thermal shock could result in brittle fracture of the rods. The $17 \%$ equivalent cladding oxidation limit (equivalency based upon stoichiometric estimates of oxidation using the Baker-Just oxidation relationships) of the AC is designed to prevent oxidation induced embrittlement. However, experimental observations of the influence of metal-water reaction induced oxidation on cladding strength (cf., Hobson and Rittenhouse, 1972) suggest that the AC limits do not assure ductile response of the cladding. The results imply that the oxidation considered acceptable under the criteria may leave the cladding in a partially ductile condition so that some fraction of the rods exposed to the limiting conditions may suffer brittle failure during quenching, at temperatures at or below $900^{\circ} \mathrm{F}$ (cf., Finlayson, 1975, Appendix 7). Thus the AC limits of $2200^{\circ} \mathrm{F}$ and $17 \%$ equivalent stoichiometric oxidation of the cladding do not provide assurance that the rods will be sufficiently ductile under these loads. The most that can be said with confidence is that if the rods reach the limiting conditions permitted under the $\mathrm{AC}$, experiments indicate some probability that embrittlement may substantially increase the probability of failure during the 
quenching and cooldown processes. A substantial reduction in the probability of brittle failure would be achieved by reducing the limits to approximately $12 \%$ equivalent stoichiometric oxidation of the cladding thickness (Finlayson, 1975, Appendix 7).

Oscillatory fluid motion in the core and downcomer during the reflood period may contribute additional loading problems to oxidation embrittled cladding. Fluid oscillations may occur as the ECC fluid attempts to enter the core. Steam binding may inhibit coolant penetration of the core until a relatively large head of standing water is achieved in the downcomer. At some fluid height in the downcomer, manometerlike fluid oscillations are expected to occur. The possibility of oscillatory loading is not specifically treated in the criteria. In fact, the inclusion of what might prove to be potentially beneficial effects of the oscillatory motion on heat transfer is specifically excluded by the criteria, as will be discussed subsequently. The omission of oscillatory loading of the core and fuel elements from the criteria, though second-order in relative importance, is potentially nonconservative, since such loadings may contribute to brittle cladding failure.

The most important hydrodynamic feature of the coolant behavior during the reflood period is, of course, the reflood rate. As previously indicated, analysis of the steam binding effect has caused estimates of reflood rates to fall to hazardously low values, of the order of $1 \mathrm{in} / \mathrm{sec}$. The factors contributing to steam binding are droplet "carryover" through the core into the steam generators, as a result of intense reflood boiling in the lower portions of the core. The droplets are boiled to steam and superheated as they pass through the hot steam generator. The steam generators, pipes, and pumps, as well as the reflooding fluid in the core itself, act as restrictions to flow of the expanding steam, which produce the general increase in system pressure associated with the steam binding. As previously noted, the increased pressure acts as a negative system feedback mechanism, reducing the reflooding rate, which reduces carryover, etc.

The AC require that droplet carryover be estimated on the basis of PWR-FLECHT experiments (Codek et al., 1971). These were extensive, but controversial, tests on reflooding in fulllength, electrically heated rod bundles. It is probable that the one-dimensional nature of the tests produced an overestimate of the amount of "carryover" fluid that would be expected in the open lattice core of an actual PWR. Overprediction of carryover is probably conservative in estimating reflood rates, as it should result in overprediction of steam generation and hence increased steam binding and decreased reflooding rates. The effects of oscillatory flow have been excluded in evaluating carryover. The effect of such neglect on conservatism is uncertain.

In evaluating steam binding, the $\mathrm{AC}$ require that the effect of steam generators on superheating the "carryover" produced steam be accounted for. Such a prescription provides adequate consideration of the effect. Also, in evaluating the influence of the pumps as restrictions, the $\mathrm{AC}$ have been prescribed conservatively. They require that the pumps be assumed to have locked impellers-unless a calculation shows that a free-running impeller would induce higher peak cladding temperatures. Whichever mode of pump operation gives the highest temperatures must be used in the peak cladding temperature calculation. Though this criterion is clearly designed to be conservative, data on pump performance under these conditions is weak. Consequently the proposed models of pump impedance are currently uncertain.

There are two primary problems associated with evaluation of the reflooding rate. The first is a very fundamental problem. The AC require that the reflood rate be calculated by an "acceptable" model. Unfortunately the supporting evidence for model adequacy is weak. As will be discussed in more detail subsequently, the coupled system thermal-hydraulic codes used in evaluating reflooding are far from having demonstrated validity. Consequently it is difficult to be sure that reflood rates are being calculated adequately. This may be a serious problem, since the current estimates give critically low reflooding rates.

The second major problem with reflooding rate estimates is associated with the potential for steam generator tube leaks and ruptures. It has been stated that tube breaks with an area of only $0.003 \mathrm{ft}^{2}$ would reduce flooding rates by $17 \%$ (Brockett $\boldsymbol{e t}$ al., 1972). At the current low flooding rates predicted for PWRs, even a minor break of this sort might reduce the rates to values so low that the core would not be adequately cooled. Thus the potential for steam generator tube leakage appears to be a serious problem which was precluded from evaluation at the ECCS hearings. The problem will no doubt be a topic for future review in connection with licensing of new reactors.

As discussed in more detail in the section on Blowdown Period Phenomena, blockage induced by swollen and ruptured rods can be a significant contributor to core flow distribution problems. In the reflood period, consideration of blockage and radial flow effects is only required by the $\mathrm{AC}$ when the reflood rates are less than $1 \mathrm{in} / \mathrm{sec}$. Then cooling must be evaluated as though there were only steam in the core. Though this requirement was clearly an attempt to introduce conservatism into the criteria, the heat transfer at reflood rates less than $1 \mathrm{in} / \mathrm{sec}$ is so poor that such rates probably should simply have been disallowed, as a more conservative criterion.

The core thermal behavior in the reflood period represents a most critical problem area in the thermal history of the core. Both the PWR and BWR depend upon experimental results of the "FLECHT" programs to provide an empirical base for the heat transfer coefficients to be used during the reflood period. The FLECHT programs were the subject of substantial controversy during the ECCS hearings. Space does not permit a thorough presentation of the associated claims and counter-claims. However, it should be recognized that both PWR-FLECHT and BWR-FLECHT programs had many problems and weaknesses. Critical tests were marred by problems with malfunctioning test equipment, indeterminate and/or poorly evaluated data, and inadequacies in the analysis of results. Consequently, instead of providing final resolution of the questions associated with reflooding and core spray heat transfer, the tests have produced almost unending controversy. On balance, however, in spite of the controversy, the results are complete enough to provide at least an adequate description of the reflood heat transfer processes.

For reflood rates greater than $1 \mathrm{in} / \mathrm{sec}$, the AC require that PWR heat transfer be based upon the PWR-FLECHT results (Codek et al., 1971). In their opinion on the AC, the Commission noted that although approving the use of PWR-FLECHT data in reflood heat transfer calculations, they felt required to observe that the results obtained with this data would represent "more nearly" best estimate calculations than bounding calculations. They concluded that an allowance of "up to $20 \%$ " might be needed to bound the data "due to experimental and inferential errors" (AEC Commissioners, 1974, p. 1124).

The reflooding rate has a very critical effect upon heat transfer. Figure 19 summarizes the results of the PWR-FLECHT program in terms of the effects of flooding rate. It can be seen that for flooding rates less than $2 \mathrm{in} / \mathrm{sec}$, the reflooding process is dramatically less effective than for flooding rates greater than $6 \mathrm{in} / \mathrm{sec}$. In fact, for flooding rates less than $1 \mathrm{in} / \mathrm{sec}$, temperature turnaround was achieved with great difficulty, if it could be achieved at all.

For reflood rates less than $1 \mathrm{in} / \mathrm{sec}$, the $\mathrm{AC}$ requires that the heat transfer analysis be conducted on the assumption that cooling is by steam only, taking into account any flow blockage due to swelling and rupture of the cladding. Although there is some question as to whether there is sufficient data to support an analysis of the core under conditions of convection cooling by steam only, the basic approach is probably adequately conservative as a method of estimating the heat transfer process. In view of the FLECHT results for reflooding rates less than 1 
FIG. 19. Summary of PWRFLECHT results as a function of flooding rate: effect on temperature rise, turnaround time, and quench time (from Codek et al., 1971).
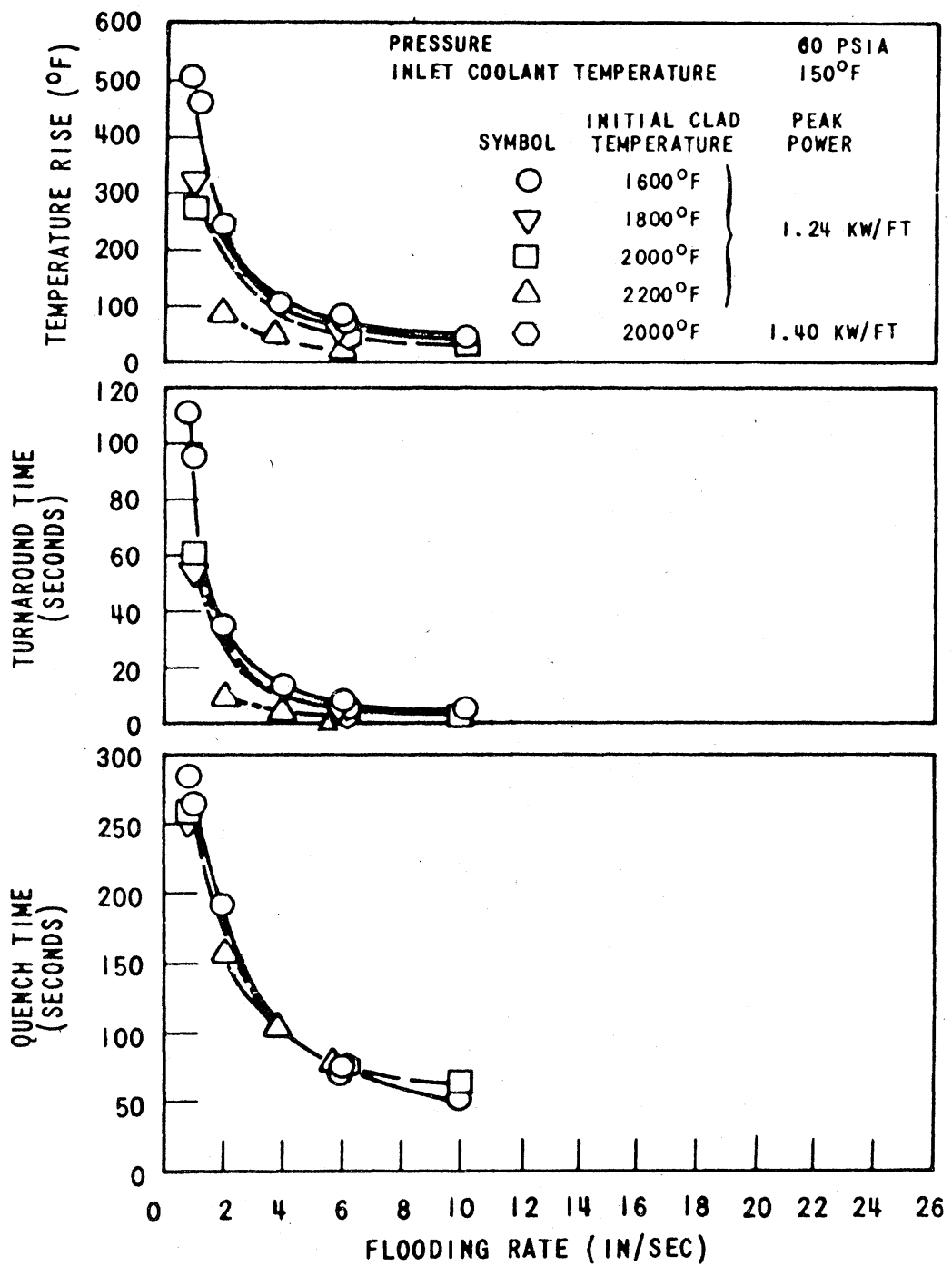

in/sec, there is reason to believe that a more acceptable criterion might simply have been to exclude consideration of rates less than $1 \mathrm{in} / \mathrm{sec}$ as being simply unacceptably low reflooding rates.

In estimating the reflood heat transfer, FLECHT test results have indicated that heat transfer coefficients increase with increasing containment pressure. Though this is not as prominent an effect in improving heat transfer rates as increasing the reflood rate, it is at least a beneficial second-order effect. The AC require that the lowest calculable pressure be used for estimating heat transfer during the reflood period, considering the effects of all installed pressure-reducing systems and processes within the containment structure. Such a requirement is definitely conservative. However, the precise amount of improvement in heat transfer with increasing pressure is somewhat uncertain as a result of a paucity of supporting data.

As previously noted, the BWR heat transfer models for core spray and reflood are based empirically on the results of the BWR-FLECHT tests (Duncan and Leonard, 1971). Essentially the same criticisms can be made of the BWR-FLECHT program as were made for PWR-FLECHT. Again, however, without going to extended discussions of FLECHT results (cf., Finlayson, 1975, Appendix 8), the program (in spite of numerous problems) has provided sufficient data to support a verdict of "adequacy" for the quality of core spray and reflood heat transfer coefficients derived from the tests. The Commission, in its opinion on the AC, acknowledged problems with the statis- tical data for BWR-FLECHT, but also confirmed the "reasonable" nature of the FLECHT specified values. They said: "The values obtained [FLECHT derived heat transfer coefficients] have always been known to have a high statistical error; furthermore the values are low and reasonable, and there seems little to be gained by renewing the controversy over the manner of conducting and interpreting all features of the test.... The high but inevitable statistical error of the coefficients of the inner rods $\left(1.5 \pm 1.0 \mathrm{BTU} / \mathrm{hr}-\mathrm{ft}^{2}-{ }^{\circ} \mathrm{F}\right)$ is bothersome and leads to an estimated error band of as much as $\pm 200^{\circ} \mathrm{F}$ in the calculated peak temperature in some circumstances (AEC Commissioners, 1974, p. 1126).

In spite of these uncertainties, the values specified do look "low and reasonable" and the heat transfer is about what would be expected for natural convection and radiation to steam. Furthermore, the heat transfer coefficients specified are those associated with cooling at atmospheric pressure, while actual conditions in the reactor may be expected to be at somewhat higher pressures, where heat transfer processes would be improved. Thus calculations based upon FLECHT results are felt to be adequate in conservatism.

\section{Adequacy of analytical methods}

Review of the elements of Table XXXII shows a considerable effort on the part of the Commission and AEC staff to develop conservative Acceptance Criteria for the ECCS. The listed 
phenomena demonstrate attempts to prescribe the individual separate effects of the LOCA in a conservative manner. The difficult question to answer is whether the individually conservatively prescribed relationships for the LOCA separate effects can yield a conservative systems analysis. At present there is no experimental data which can demonstrate unequivocally that the integrated systems effects are conservatively prescribed, even if all the individual pieces are independently conservative. Therefore any meaningful evaluation of systems effectiveness, or the conservatism of its evaluation, must currently depend upon the adequacy of the systems analysis codes.

Considerable reliance is placed on computer codes to predict the course of postulated reactor accidents-particularly the LOCA. These codes have not been verified by substantive comparison with experimental data because the data required do not yet exist. (A number of separate effects and systems tests, culminating in LOFT, are currently underway or planned.) Because the codes have not been thoroughly verified, the precise quantitative predictions of accident behavior must be regarded as uncertain. In licensing evaluation, attempts are made to compensate for uncertainties by introducing the "conservative" assumptions of the $\mathrm{AC}$, which have been designed to yield reasonable upper limits on the predicted accident severity. Much of the controversy in LWR safety is derived from differing opinions about the degree of conservatism introduced by the Criteria.

In order to test the validity of the codes, and ultimately to improve their realism, the $\mathrm{AEC}$ has recently instituted a "standard problem" approach in which each of the reactor manufacturers, fuel suppliers, and Aerojet Nuclear Co. have been asked to predict the results of experiments, prior to their performance (or at least without prior knowledge of the results). The predictions made with the LOCA/ECCS analysis codes are then compared in detail with the experimental results.

To date, three such comparisons have been made. The first such experiment, which consisted of the rapid blowdown of a simple straight pipe performed by Edwards and O'Brien (1970) in England, is about as simple and undemanding a test as could reasonably be envisioned for codes intended to predict the complex events during and following blowdown of a power reactor. The results of all participants were reasonably good, but by no means perfect (Gamer, 1973). The inadequacy of the results is particularly significant when it is observed that the participants were given results of the test in advance of the performance of

\section{Core Inlet Flow}

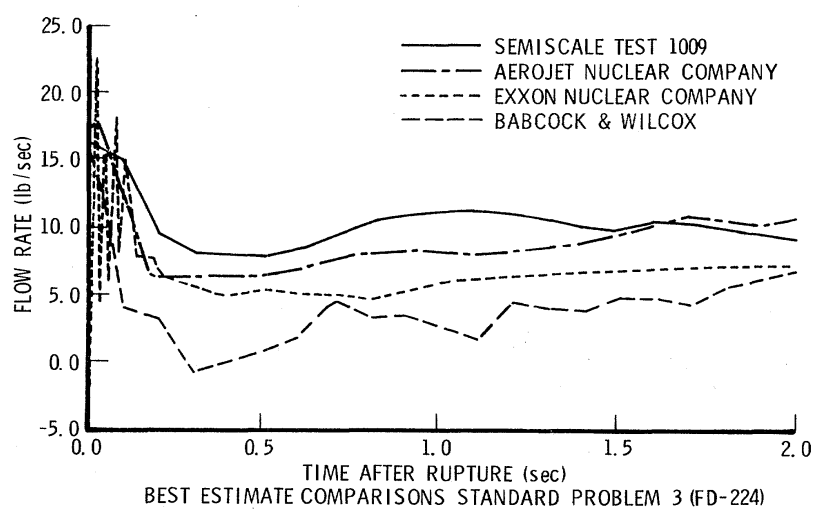

FIG. 20. Core inlet flow. their calculations and allowed to manipulate their codes to attain the best possible solutions.

The second and third experiments consisted of blowdowns of an apparatus at the INEL, known as 1-1/2 Loop Semiscale. The Semiscale apparatus is an approximated $1 / 30$ scale representation of the LOFT reactor, and is essentially one dimensional. While Semiscale will ultimately incorporate an electrical simulation of a reactor core, the standard problem tests did not employ a heat source. Consequently, at initiation of the blowdown, the water was at essentially uniform temperature throughout the apparatus. Thus, Semiscale blowdowns, while providing a considerably more rigorous test of the codes than the simple pipe blowdown, were still considerably less complex than those which would occur in a reactor blowdown, (i.e., one-dimensional, no heat input during blowdown, etc., and in one test, no ECC injection). Typical comparisons of the (unheated) core inlet flow and the downcomer flow (two of the most important variables), as measured during the experiment and computed by several best estimate models, are shown in Figs. 20 and 21 . The results are not encouraging, and tend to indicate that the physical processes are not well modelled. ${ }^{21}$

The lack of agreement ${ }^{22}$ between prediction and experimental results for systems which are simple in comparison to a large power reactor, casts considerable doubt on the ability of the codes to predict the course of a LOCA realistically. Further, because the lack of agreement indicates that the important physical processes involved are apparently improperly modelled in the codes, the "conservatism" produced by the evaluation models (which are identical to the best estimate models but employ "conservative" inputs) cannot be proven.

\section{Downcomer Flow}

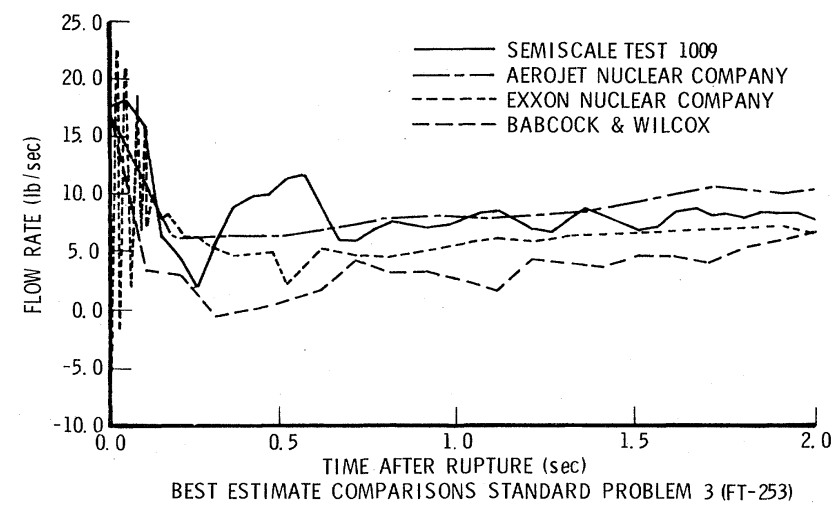

FIG. 21. Downcomer flow.

\footnotetext{
${ }^{21}$ During discussion with AEC personnel and others, repeated implications were made that the vendor codes were superior to the RELAP code developed for the AEC by Aerojet. The standard problem comparisons do not appear to support their view, in that RELAP (Aerojet) appears to yield results which are as good or better than those produced by any of the other participants.

${ }^{22}$ The only reason given for the lack of agreement was that, because of time and budget pressures, best efforts were not employed in preparing input for the various codes. Thus it appears that the standard problem approach, which is essential to the improvement and verification of the codes, is not being seriously pursued by the industry. If this is indeed the case, then the entire AEC approach to the resolution of the LOCA problems must be re-examined.
} 


\section{APPENDIX II: CONTAINMENT AND CONSEQUENCES}

In this Appendix we provide basic data, analyses, and references for the discussion of Sec. V. Specifically, we present an independent calculation ${ }^{23}$ of the numbers of cancers, genetic defects, and nonfatal thyroid cancers from a particular major release of radioactivity. We also estimate the magnitude of costs associated with ground contamination following a core melt accident at a nuclear power plant. The release of radioactivity we consider is described in Draft WASH-1400 as the "reference accident" or PWR2, and is the accident for which the most data are given. In order to provide as transparent a calculation as possible, we use a simple approximations to the dispersion and deposition of the radioactivity, and we then attempt to verify these results by comparison with detailed results of Draft WASH-1400 in areas of overlap. We are then in a position to estimate the magnitude of some effects which were not calculated in Draft WASH-1400. We find that some of these effects would be large and would indeed be the dominant longterm effects on health in the reference accident. The most important of such neglected effects are the consequences of the long-term radiation dose received by the population at large distances from such a major reactor accident, resulting in a small probability of cancer per person for a large number of people. We then discuss means for mitigating such potential consequences of reactor accidents.

\section{A. Radioactive release from core}

\section{Time scale}

For specificity, we discuss the PWR reactor considered in Draft WASH-1400 (Table XXXIII).

Taking specific heats of $0.07,0.07$, and $0.12 \mathrm{BTU} / \mathrm{lb}^{\circ} \mathrm{F}$ for $\mathrm{UO}_{2}$, Zircaloy, and steel, respectively (Glasstone and Sesonske, $1967, \mathrm{p} .814)$, the heat capacity of the core is approximately $16000 \mathrm{BTU} /{ }^{\circ} \mathrm{F}$. The ANS standard heat rate from fission products is,

$$
\begin{aligned}
P / P_{0} & =0.0766 t^{-0.181} \quad 10<t<150 \mathrm{sec} \\
& =0.130 t^{-0.283} \quad 150<t<4 \times 10^{6} \mathrm{sec},
\end{aligned}
$$

where $t=0$ is the time when the fission chain reaction stops, i.e., when the reactor is "turned off" (Draft WASH-1400, Appendix VIII, pp.A-2, A-3). This heat rate is valid for a reactor in which all fission products have reached their equilibrium levels. This approximation is good to within $25 \%$ until one hour after shutdown for a reactor which has been operating at power for at least a week (Glasstone and Sesonske, 1967, p. 101). This averages to approximately $2 \%$ over the first hour. To this

\footnotetext{
${ }^{23}$ In order to reduce the risk of publishing a report containing major misunderstandings, we submitted in writing to personnel of the Nuclear Regulatory Commission a set of questions covering the technical basis for the differences in our result from those of Draft WASH-1400. We received replies to these questions at a meeting in Washington, March 17, 1975, and in a communication, dated March 27, 1975. NRC is working to convert Draft WASH-1400 to a final report, and many refinements and corrections have been addressed by the group doing this work. Several of the discrepancies between our results and those of Draft WASH-1400 calculated for the same assumptions turn out to arise from a lack of consistency between the input to the computer program used in deriving the results of Draft WASH-1400 and the text of the Draft itself. In general, we continue to refer to the data presented in Draft WASH-1400, calling attention to important changes that have been called to our attention by the indication, "(NRC, 1975a)" or "(NRC, 1975b)." Draft WASH-1400 was a Draft, and we hope that our work will help the authors in their efforts to produce as accurate and authoritative a document as possible.
}

TABLE XXXIII. PWR data (selected from Draft WASH-1400, Appendix VIII, pp. 53-56).

\begin{tabular}{lr}
\hline \hline Nominal power & $\begin{array}{r}2441 \mathrm{mw} \text { (thermal) } \\
\left(8331 \times 10^{6} \mathrm{BTU} / \mathrm{hr}\right)\end{array}$ \\
\hline Core weight (pounds) & \\
$\mathrm{UO}_{2}$ & 175600 \\
Zircaloy & 36300 \\
Miscellaneous & \\
$\quad$ (We assume steel) & 14300 \\
\hline \hline
\end{tabular}

must be added perhaps another $0.5 \%$ due to the decay of actinide isotopes made radioactive by neutron capture. Two and a half percent of $8.3 \times 10^{9} \mathrm{BTU} /$ hour is $5.8 \times 10^{4} \mathrm{BTU} / \mathrm{sec}$, corresponding to an adiabatic heatup rate of the core of $3.6^{\circ} \mathrm{F} / \mathrm{sec}$. Thus in a loss-of-coolant accident without emergency core cooling it would take the core approximately 18 minutes to go adiabatically from an average temperature of $1000^{\circ}$ to $5000^{\circ} \mathrm{F}$, the melting temperature of $\mathrm{UO}_{2}$.

\section{Radioactive inventory}

We have divided the radioactive inventory of the core into groups according to chemical and biological properties. Table XXXIV gives a partial shutdown inventory of isotopes which have half-lives of the order of one hour or longer, for a $1000 \mathrm{MWe}$ PWR with an average fuel exposure of $17.6 \mathrm{MWday} / \mathrm{kg}$ (Draft WASH-1400, Appendix VI, pp. 6, 60).

\section{Carrying capacity of the containment atmosphere}

The weight of the air in a $1.8 \times 10^{6} \mathrm{ft}^{3}$ PWR containment volume is approximately 50 tons at Standard Temperature and pressure (STP); and the fissile content of the reactor core never exceeds 3 tons. There is thus no question of volume considerations limiting the release of the fission products such as $\mathrm{Xe}, \mathrm{Kr}$, and I which will be in gaseous or vapor form. The other fission products with higher boiling temperatures would exist as aerosols, however, so we must turn to the question of the weight of aerosols which could be suspended in this volume.

The Stokes settling velocity

$$
v=\rho g d^{2} / 18 \eta
$$

for a spherical particle of diameter $d=2 \mu$ and with a density $\rho$ of 8 (steel) is about $0.1 \mathrm{~cm} / \mathrm{sec}\left(\eta_{\text {air }} \approx 2 \times 10^{-4} \mathrm{gm} / \mathrm{cm}-\mathrm{sec}\right)$. In 1000 seconds such a particle would "fall" one meter. Since the Stokes settling velocity varies as the square of the diameter, one would expect an aerosol of smaller particles to remain suspended for a long time.

Aerosol particles also coagulate as the particles undergo collision in the gas phase. The number density, $n$, of an aerosol of identical particles with radii of the order of one micron will decrease due to coagulation approximately as

$$
d n / d t=-K_{0} n^{2}=0.5 \times 10^{-9} n^{2}
$$

(Green and Lane, 1964, Chap. 5), with the solution

$$
1 / n-1 / n_{0}=0.5 \times 10^{-9} t \text {. }
$$

(In Eq. AII.3, a simple tîeory gives $K_{0}=4 k T / 3 \eta$, with $\eta$ the viscosity of air. The numerical value for $K_{0}$ includes some small corrections). For any large $n_{0}$ (i.e., $n_{0}>10^{9}$ ), $n$ will, in a few seconds, become independent of $n_{0}$, and the number of aerosol particles per $\mathrm{cm}^{3}$ will decrease like

$$
n \approx 2 \times 10^{9} / t \text {. }
$$

For $t$ of the order of 1000 seconds, we have $n$ about $2 \times 10^{6}$ particles $/ \mathrm{cm}^{3}$.

Thus it is possible for the containment to be filled with par- 
ticles of the order of about 2 micron diameter with a density of about $2 \times 10^{6} / \mathrm{cm}^{3}$. For a particle mass density of $8 \mathrm{~g} / \mathrm{cm}^{3}$, this would come to two tons or approximately two percent of the total mass of one PWR core.

It is instructive to calculate how long it would take to evaporate this much mass once the core is molten. Iron boils at $5400^{\circ} \mathrm{F}$ and has a heat of vaporization of $2700 \mathrm{BTU} /$ pound. Decay heat at the rate 2.5 percent of full power could boil off steel at the rate of 40 tons per hour. Obviously, there is plenty of heat available to generate the aerosol.

If the containment atmosphere spray and filtration systems function, the loading of the containment atmosphere with radionuclides other than the noble gases could be reduced by an order of magnitude or so (Draft WASH-1400, Appendix VII, pp. 26-53).

\section{B. Atmospheric dispersion}

\section{Containment failure time and rate of release}

If one of the series of failures that would lead to loss of coolant and meltdown were to occur, the containment would be breached within a period of the order of a day. In a large PWR containment, if the containment atmosphere cooling and isolation systems work, the failure will be a result of the molten core's penetrating the concrete base mat of the containment, and the release would occur into and through the soil under the containment. Other containment failure modes would release the containment atmosphere directly into the outside atmosphere. This could occur as a result of:

- failure of containment isolation valves;

- a "steam explosion" violent enough to rupture the containment; or

- the overpressuring of the containment by steam or noncondensible gases. Below we offer a simplified calculation of the energetics involved in the case of overpressure by steam.

The threshold assumed for containment failure in Draft WASH-1400, Appendix VII, is 100 psi. The energy required to generate a cubic foot of saturated steam at this pressure is approximately $250 \mathrm{BTU} / \mathrm{ft}^{3}{ }^{3}$ For the full PWR containment volume, this corresponds to about $0.45 \times 10^{9} \mathrm{BTU}$. The stored heat in the primary coolant water and core is of the order of $0.25 \times 10^{9}$ BTU (Draft WASH-1400, Appendix VIII, p. 53) and, at $2.5 \%$ decay heat, the core is producing of the order of $0.21 \times 10^{9} \mathrm{BTU} / \mathrm{hr}$. Complete oxidation of the zirconium in the core at $4200 \mathrm{BTU} /$ pound will release a total of $0.150 \times 10^{9} \mathrm{BTU}$.

Therefore, in the absence of effective containment heat removal, one would expect the PWR containment to fail due to overpressure (i.e., if it has not already failed as a result of a steam explosion or failure of the isolation valves) within a period of the order of an hour. This result is consistent with that in Draft WASH-1400, Appendix VIII. A small BWR containment could be overpressured by noncondensible gases in a similar length of time.

\section{The plume}

There are several meterological factors that govern the dispersion of the radioactivity after an assumed breach of containment.

\section{a. Plume rise}

Because the atmosphere in the containment is hot, the released plume would be buoyant. The radioactivity carried by the plume would add more heat. Gifford (1967) has derived a simplified plume-rise equation from these considerations and the assumption that the plume radius grows with height as
TABLE XXXIV. Radioactive inventory at shutdown of water reactor (from Draft WASH-1400, Appendix VI, pp. 6, 60).

\begin{tabular}{|c|c|c|}
\hline Is otope & $\begin{array}{l}\text { Half-life } \\
\text { days } \quad \text { (years) }\end{array}$ & $\begin{array}{c}\text { Inventory } \\
\left(10^{6} \text { curies }\right)\end{array}$ \\
\hline \multicolumn{3}{|l|}{ Noble gases } \\
\hline${ }^{85} \mathrm{Kr}$ & $10.76(\mathrm{yr})$ & 0.6 \\
\hline${ }^{85} \mathrm{Kr}$ & 0.18 & 26 \\
\hline${ }^{87} \mathrm{Kr}$ & 0.053 & 52 \\
\hline${ }^{88} \mathrm{Kr}$ & 0.116 & 76 \\
\hline${ }^{133} \mathrm{Xe}$ & 5.3 & 170 \\
\hline${ }^{135} \mathrm{Xe}$ & 0.38 & 26 \\
\hline \multicolumn{3}{|l|}{ Iodines } \\
\hline${ }^{131} \mathrm{I}$ & 8.05 & 85 \\
\hline${ }^{132} \mathrm{I}$ & 0.1 & 120 \\
\hline${ }^{133} \mathrm{I}$ & 0.875 & 170 \\
\hline${ }^{134} \mathrm{I}$ & 0.036 & 200 \\
\hline${ }^{135} \mathrm{I}$ & 0.28 & 150 \\
\hline \multicolumn{3}{|l|}{ Telluriums } \\
\hline${ }^{129} \mathrm{Te}$ & 0.048 & 28 \\
\hline${ }^{129} \mathrm{Te}^{*}$ & 34.1 & 10 \\
\hline${ }^{131} \mathrm{Te}^{*}$ & $1: 25$ & 15 \\
\hline${ }^{132} \mathrm{Te}$ & 3.25 & 120 \\
\hline \multicolumn{3}{|l|}{ Cesiums } \\
\hline${ }^{134} \mathrm{Cs}$ & $2(\mathrm{yr})$ & 1.7 \\
\hline${ }^{136} \mathrm{Cs}$ & 12.9 & 6 \\
\hline${ }^{137} \mathrm{Cs}$ & $30(\mathrm{yr})$ & 5.8 \\
\hline \multicolumn{3}{|l|}{ Volatile oxides } \\
\hline${ }^{99} \mathrm{Mo}$ & 2.8 & 160 \\
\hline${ }^{99} \mathrm{Tc}$ & 0.25 & 140 \\
\hline${ }^{103} \mathrm{Ru}$ & 40 & 100 \\
\hline${ }^{105} \mathrm{Ru}$ & 0.18 & 58 \\
\hline${ }^{106} \mathrm{Ru}$ & $1(\mathrm{yr})$ & 19 \\
\hline${ }^{105} \mathrm{Rh}$ & 1.5 & 58 \\
\hline \multicolumn{3}{|c|}{ Alkaline earths } \\
\hline${ }^{140} \mathrm{Ba}$ & 12.8 & 160 \\
\hline${ }^{89} \mathrm{Sr}$ & 50.6 & 110 \\
\hline${ }^{90} \mathrm{Sr}$ & $27.7(\mathrm{yr})$ & 5.2 \\
\hline${ }^{91} \mathrm{Sr}$ & 0.4 & 130 \\
\hline \multicolumn{3}{|c|}{ Nonvolatile oxides } \\
\hline${ }^{90} \mathrm{Y}$ & 2.7 & 5.2 \\
\hline${ }^{91} \mathrm{Y}$ & 59 & 140 \\
\hline${ }^{95} \mathrm{Zr}$ & 65.5 & 160 \\
\hline${ }^{97} \mathrm{Zr}$ & 0.7 & 160 \\
\hline${ }^{95} \mathrm{Nb}$ & 3.5 & 160 \\
\hline${ }^{140} \mathrm{La}$ & 1.66 & 160 \\
\hline${ }^{141} \mathrm{Ce}$ & 32.8 & 160 \\
\hline${ }^{143} \mathrm{Ce}$ & 1.37 & 150 \\
\hline${ }^{144} \mathrm{Ce}$ & 285 & 110 \\
\hline${ }^{143} \mathrm{Pr}$ & 13.6 & 150 \\
\hline${ }^{147} \mathrm{Nb}$ & 11 & 60 \\
\hline${ }^{147} \mathrm{Pm}$ & $2.65(\mathrm{yr})$ & 17 \\
\hline${ }^{149} \mathrm{Pm}$ & 2.2 & 40 \\
\hline${ }^{238} \mathrm{Pm}$ & $86.4(\mathrm{yr})$ & 0.1 \\
\hline${ }^{239} \mathrm{Pm}$ & $24390(\mathrm{yr})$ & 0.01 \\
\hline
\end{tabular}

$$
r=\epsilon h, \epsilon=0.44,
$$

where $h$ is the height to which the plume has risen. The resulting equation may be written as

$$
d^{2} s / d t^{2}+\beta s-3(F+F * t) / \epsilon^{2} u=0,
$$

where

$$
s=h^{3}
$$


$u$ equals the wind velocity, and

$$
\beta=(g / T)[(d T / d h)+9.8] \text {. }
$$

In Eq. (AII.4) $g$ is the gravitational constant and $T$ the atmospheric temperature in ${ }^{\circ} \mathrm{K}$. The adiabatic lapse rate (i.e., rate of decrease of temperature with altitude) for dry air is $d T / d h$ $=-9.8^{\circ} \mathrm{C} / \mathrm{km}$. In the plume equation

$$
F=g G / \pi \rho C_{p} T, F *=g G * / \pi \rho C_{p} T,
$$

where $G$ is the rate at which the plume carries heat away from the containment, $G *$ is the rate at which heat is added by radioactivity to a segment of the plume which has been released in a second, and $C_{p}$ is the heat capacity of air per unit volume at STP. For initial conditions, $h=d h / d t=0$ at $t=0$, and $\beta>0$ (stable atmosphere) the solution to the plume-rise equation is

$$
h^{3}=\left(3 / \beta \epsilon^{2} u\right)\left\{F\left(1-\cos \beta^{1 / 2} t\right)+F * t\left[1-\sin \left(\beta^{1 / 2} t\right) /\left(\beta^{1 / 2} t\right)\right]\right\} .
$$

The sinusoidal terms are a reflection of the physically unrealistic assumption implicit in Eq. AII.6-that the plumes shrink when they sink. They should therefore be taken only as an indicator of time scale for plume rise. For an isothermal atmosphere with $u=5 \mathrm{~m} / \mathrm{sec}(11$ miles $/ \mathrm{hr})$, and a containment release over a period of 1 hour of a saturated steam atmosphere at 100 psi carrying radioactivity equivalent to $0.5 \%$ of full power, the solution of Eq. AII.11, neglecting the sinusoidal terms, is

$$
h=170\left(1+2.6 \times 10^{-5} t\right)^{1 / 3} \text { meters, }
$$

with an initial rise time to 170 meter height of the order of

$$
\beta^{-1 / 2}=55 \text { sec }
$$

\section{b. Vertical dispersion}

There is a question whether a plume released at ground level would actually rise in the above fashion. For this reason plume rise from ground-level releases has been treated in Draft WASH-1400, Appendix VI, as a contributor to the vertical dispersion in the exposure formula,

$$
\chi=Q \exp \left(-z^{2} / 2 \sigma_{z}{ }^{2}\right) /\left[\theta(\pi / 2)^{1 / 2} \sigma_{z} u\right] .
$$

Here $Q$ is the total amount of radioactivity in the plume (curies), $\theta$ is the plume width in radians, $u$ is the wind velocity,

$$
\sigma_{z}=\left[\left(\sigma_{z}{ }^{A}\right)^{2}+h^{2}\right]^{1 / 2}
$$

(where $\sigma_{z}{ }^{A}$ is a dispersion parameter associated with the atmospheric stability), and $\chi$ is a measure of integrated exposure to the plume (measured in curies $-\mathrm{sec} / \mathrm{m}^{3}$ ). Terms associated with cloud depletion by radioactive decay and deposition are not shown. ${ }^{24}$

\section{c. The wedge model}

Immediate health effects associated with high doses of radiation would be confined to within a few tens of miles from the reactor under most weather conditions. Within this distance, doses would be affected significantly by atmospheric stability, inversion heights, height of the release, and plume rise. Immediate health effects are also quite sensitive to the magnitude of the doses, since threshold phenomena are involved. For all these reasons, calculated immediate effects will be sensitive to the detailed modeling of the meterological effects, radionuclide release fractions, biological dose-response models, evacuation assumptions, etc. and are not only variable but also quite uncertain at the current level of sophistication for these calculations.

\footnotetext{
${ }^{24}$ For the lore on atmospheric dispersion estimates, see Turner (1970) and Slade (1968).
}

While it is difficult to calculate the total immediate radiation effects on health where it is necessary to calculate exact doses to specific individuals, we can compute the average total longterm man-rem dose received as a result of a given release using a simplified model, the "wedge model."

In our applications of this approximate model the distances of concern are hundreds of miles. Over this distance the plume will ordinarily have diffused fairly completely through the vertical height of the mixing layer, usually a few thousand feet. We therefore make the approximation that the concentration of the plume is uniform through this layer. Also, instead of using a Gaussian distribution for concentration in the horizontal direction cross-wind, we use (as does Draft WASH-1400) a mean constant concentration within a wedge. The effective wedge angle $\theta$ is typically 0.13 to 0.7 radians depending on prevailing atmospheric stability. The final result for man-rem population doses is found to be independent of $\theta$ and of the detailed form of the angular distribution.

Ignoring fallout and radioactive decay for the time being, it is easy to see that within the wedge $\chi(r)=Q / H u \theta r$ where $\chi$ is the time-integrated concentration in curies-sec $/ \mathrm{m}^{3}, Q$ is the total release in curies, $r$ the distance from the reactor, $H$ the mixing-layer height, and $u$ the wind velocity.

The radioactivity falls out at a rate proportional to the product of the ground level concentration and the vertical, or "deposition," velocity of the radioactivity, $v_{d}$. Assuming that the plume remains well-mixed vertically, and including the effect of depletion by fallout, we get

$$
\chi(r)=\left(Q e^{-\lambda_{f} r}\right) / H u \theta r, \text { where } \lambda_{f}=v_{d} / u H
$$

in our approximation. We shall use Draft WASH-1400 values for $v_{d}: v_{d}=0$ for noble gases, $0.5 \mathrm{~cm} / \mathrm{sec}$ for iodine, and 0.2 $\mathrm{cm} / \mathrm{sec}$ for other isotopes. These deposition velocities have been established experimentally only within an order of magnitude (Slade, 1968, Chapters 4 and 5).

Using the Draft WASH-1400 figures above, we get $\lambda_{F}{ }^{-1}=770$ $\mathrm{km}$ for iodine, and $1900 \mathrm{~km}$ for other isotopes, for $H=1100$ meters and $u=3.5 \mathrm{~m} / \mathrm{sec}$. These values of $H$ and $u$ are averages over values used in Draft WASH-1400. (See below.) This distance scale $\lambda_{F}{ }^{-1}$ is much larger than the distance beyond which the wedge model approximation to more exact Gaussian plume models becomes reasonable for most atmospheric conditions, so that there is a considerable range of distances over which the wedge model is valid.

For an isotope with decay constant $\lambda_{d},\left(\mathrm{sec}^{-1}\right)$ we have

$$
\chi(r)=(Q / u H r \theta) \exp \left[\left(-\lambda_{F}-\lambda_{d} / u\right) r\right] \text { curies }-\mathrm{sec} / \mathrm{m}^{-3} \text {. }
$$

\section{Radiation doses}

\section{a. Inhalation doses}

To get an estimate of the total population dose by inhalation to the whole body or to a specific organ (doses arising from radioactivity inhaled and retained) integrated out to a maximum distance, $R$, we have:

$$
\begin{aligned}
D=\int_{-\theta / 2}^{\theta / 2} d \theta \int_{0}^{R} & \chi(r) p b F_{\mathrm{DC}} r d r=\frac{p Q b F_{\mathrm{DC}}}{v_{d}+\lambda_{d} H} \\
& \times\left[1-\exp \left(-\lambda_{f}-\lambda_{d} / u\right) R\right] \text { man-rem, }
\end{aligned}
$$

where $p$ is the average population density, $b$ is the breathing rate, and $F_{\mathrm{DC}}$ is the dose conversion factor in rem per curie inhaled.

In Table XXXV we list some of the dose conversion factors given in Draft WASH-1400, Appendix VI, p.57. These are doses to the whole body, lungs, and GI tract due to the inhalation of a millicurie of each of the listed isotopes. (The numbers for 50-year lung doses are our own, calculated from the corresponding Draft WASH-1400 30-day numbers by a prescription which will be explained below.) 
TABLE XXXV. Doses due to inhalation of a radioactive aerosol (in rem/m curie). [ From Draft WASH-1400, Appendix VI, p. 57 (except for the 50-year lung doses)].

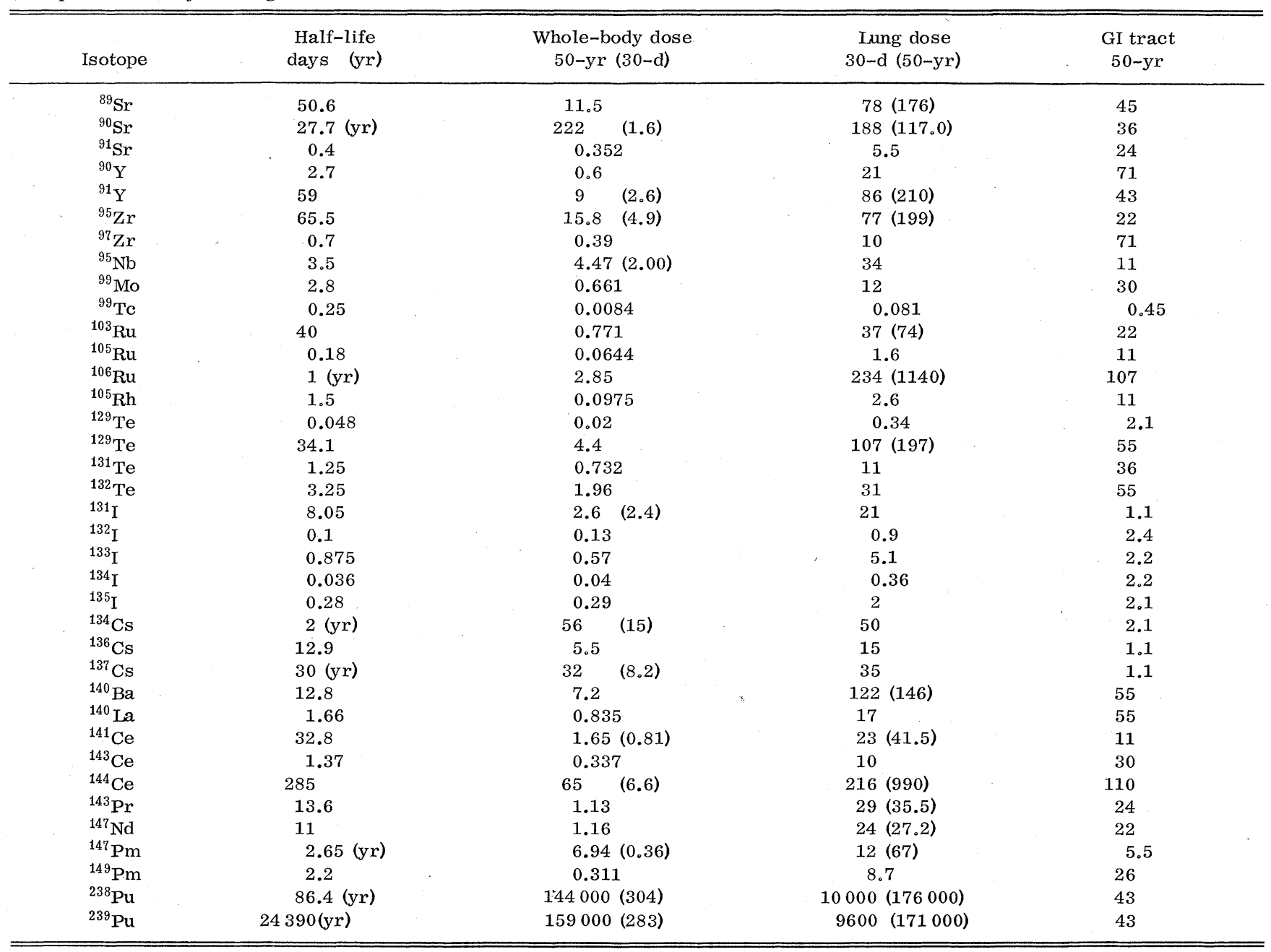

${ }^{a}$ Draft.

The lung doses were calculated using the assumptions that $25 \%$ of the aerosol inhaled is exhaled, that $50 \%$ of it is deposited in the upper respiratory passages and subsequently swallowed, and that $25 \%$ is deposited in the lower respiratory passages. Of the last $25 \%$, it is assumed that one-half the insoluble component is eliminated from the lungs and swallowed within the first 24 hours and that the other half has a residence half-life in the lungs of 120 days before being absorbed into the body (except for plutonium which is assumed to have a residence half-life of one year). ${ }^{25}$

These inhalation dose conversion factors should be used only with several reservations:

(1) They depend upon assumptions as to whether the isotopes are in soluble or insoluble form. These assumptions are not made explicit in the reference.

(2) For purposes of calculating exposures contributing to lung cancer it is inappropriate to truncate the integration at 30 days. This fact was recognized in Draft WASH-1400 in the case of the whole-body exposures where both 30-day (for acute effects) and 50-year values (for cancer) are offered where they differ

${ }^{25} \mathrm{All}$ but the last assumption for plutonium are taken from ICRP, 1959, p. 153. substantially. We have calculated the corresponding 50-year lung doses for the insoluble isotopes using the following dependence of integrated dose on integration interval $T$ :

$$
\left.D(T)=\frac{1}{8} \int_{0}^{T} d t \exp (-t / \tau)[1-t) U(1-t)+\exp \left(-t / \tau_{L}\right)\right] .
$$

Here $\tau$ is the exponential decay lifetime of the isotope corresponding to its half-life and $\tau_{L}$ is the exponential time corresponding to the biological half-life in the lungs assumed for the $1 / 8$ of the inhaled insoluble aerosol radioactivity which has the longest residence time in the lungs (120-day half-life for all insoluble compounds except plutonium where 1 year is used, Draft WASH-1400, Appendix VI, p.56). The first term in the square brackets corresponds to the dose from the $1 / 8$ of the inhaled radioactivity which is assumed to be cleared from the lungs within the first day. $(U(\chi)$ is the step function with value 1 for $\chi>0$ and 0 for $\chi<0$.) We have indicated to 50-year lung doses in Table XXXV in parentheses next to the 30-day lung doses in those cases where the difference is significant.

(3) No calculations of bone doses have been offered although this dose can be relatively very large in the long term-particularly in the case of ${ }^{90} \mathrm{Sr}$. 
TABLE XXXVI. Thyroid inhalation doses averaged over age groups (from EPA, 1973).

\begin{tabular}{|c|c|c|c|c|c|c|c|c|}
\hline \multirow[t]{2}{*}{$\begin{array}{l}\text { Age group } \\
\text { (Percentage } \\
\text { of } \\
\text { population) }\end{array}$} & \multirow[t]{2}{*}{$\begin{array}{l}\text { Fraction of } \\
\text { inhaled iodine } \\
\text { reaching } \\
\text { thyroid }\end{array}$} & \multirow[t]{2}{*}{$\begin{array}{l}\text { Biological } \\
\text { half-life } \\
\text { for iodine } \\
\text { (days) }\end{array}$} & \multirow[t]{2}{*}{$\begin{array}{l}\text { Thyroid mass } \\
\text { (grams) }\end{array}$} & \multicolumn{2}{|c|}{$\begin{array}{c}\text { Effective } \\
\text { decay energy } \\
(\mathrm{MeV})\end{array}$} & \multicolumn{2}{|c|}{$\begin{array}{c}\text { Dose conversion } \\
\text { factors } \\
\text { (rem/m curies } \\
\text { inhaled) }\end{array}$} & \multirow[t]{2}{*}{$\begin{array}{l}\text { Breathing } \\
\text { rate } \\
\text { (cc/sec) }\end{array}$} \\
\hline & & & & ${ }^{131} \mathrm{I}$ & ${ }^{133} \mathrm{I}$ & ${ }^{131} \mathrm{I}$ & ${ }^{133} \mathrm{I}$ & \\
\hline $\begin{array}{c}0-1 \text { year } \\
(1.79)\end{array}$ & 0.38 & 20 & 1.9 & 0.18 & 0.40 & $\begin{array}{r}15000 \\
(4800\end{array}$ & $\begin{array}{l}4800 \\
1200)^{a}\end{array}$ & $\begin{array}{l}37 \\
(35)^{a}\end{array}$ \\
\hline $\begin{array}{c}1-9 \text { years } \\
(16.47)\end{array}$ & 0.38 & 29 & 2.7 & 0.18 & 0.40 & $\begin{array}{r}12.000 \\
(4800\end{array}$ & $\begin{array}{l}3400 \\
1000)^{a}\end{array}$ & $\begin{array}{l}110 \\
(81)^{a}\end{array}$ \\
\hline $\begin{array}{c}10-19 \text { years } \\
(19.57)\end{array}$ & 0.28 & 70 & 12 & 0.19 & 0.42 & $\begin{array}{r}2400 \\
(1700\end{array}$ & $\begin{array}{l}600 \\
420)^{a}\end{array}$ & $\begin{array}{l}200 \\
(155)^{a}\end{array}$ \\
\hline $\begin{array}{r}20 \text { years } \\
(62.17)\end{array}$ & 0.23 & 100 & 20 & 0.19 & 0.42 & $\begin{array}{r}1200 \\
(1500\end{array}$ & $\begin{array}{l}300 \\
370)^{\text {a }}\end{array}$ & $\begin{array}{l}230 \\
(232)^{\mathrm{a}}\end{array}$ \\
\hline
\end{tabular}

${ }^{a}$ Values used in Draft WASH-1400.

\section{b. Thyroid inhalation dose}

We treat the dose to the thyroid in somewhat greater detail because this is a subject which in previous calculations has received inadequate attention. Our principle reference in an EPA analysis ${ }^{26}$ which divides the population into four groups as shown in Table XXXVI. The table also shows the age dependence of the various factors which go into computing the thyroid dose from the amount of radioactivity inhaled and the final conversion factors for ${ }^{131} \mathrm{I}$ (8-day half life) and ${ }^{133} \mathrm{I}$ (21 hours). The EPA report has neglected the thyroid doses from the shorter-lived iodine isotopes: ${ }^{132}$ I ( 2 hours), ${ }^{134}$ I (1 hour), ${ }^{135}$ I (6.5 hours). This seems to be a reasonable approximation. (In our calculations we have simply taken the child dose conversion factors for these isotopes equal to the ICRP adult dose conversion factors.)

The EPA dose conversion factors differ somewhat from the factors used in Draft WASH-1400. We have learned that the latter report uses figures taken from a previous AEC study, WASH-1258, 1973, Vol. 1, p.6B-35. The agreement between the EPA and these Draft WASH-1400 thyroid dose conversion factors is within $30 \%$ except in the case of children aged 1-9 where the EPA dose conversion factors are about 2.5-three times larger. Part of this discrepancy (a factor of about 1.7) seems to be associated with the assumption in WASH-1258 (p.6B-10) that the fraction of the inhaled iodine reaching the thyroid is the same for children as for adults. Experiments indicate a wide spectrum of fractional uptakes for children. ${ }^{27}$ Not enough information is offered in WASH-1258 for us to determine the source of the remaining discrepancy.

We note, however, that in the AEC study entitled, The Potential Radiological Implications of Nuclear Facilities in the Upper Mississippi River Basin in the Year 2000, WASH-1209, 1973 , the mass of the "four-year-old child's thyroid" is given as 5 grams (p. IX-4), whereas the value used by the EPA is only half as large (see Table XXXVI). The computed dose varies roughly in inverse proportion to this mass. The only study of which we are aware, Mochizuki et al. (1963), gives the following thyroid mass averages: $1-2$ years, $1.8 \pm 0.6 \mathrm{gm}$; $3-4$ years, $2.6 \pm 0.9 \mathrm{gm} ; 5-9$ years, $4.6 \pm 1.5 \mathrm{gms}$.

The breathing rate for children used in WASH-1258 is also smaller than the EPA number by a factor 1.35. The net result is that the child thyroid dose from radioiodine inhalation computed with the AEC numbers would be 3.5 times smaller than those computed by the EPA prescription.

We are not in a position to resolve this discrepancy. We

\footnotetext{
${ }^{26}$ Our principal reference here is the detailed discussion presented in EPA, 1973, pp. 133-118.

${ }^{27}$ See the summary by Cole (1972), Appendix E.
}

will, therefore, treat the AEC and EPA dose conversion factors as the limits of a range of uncertainty. The subject should obviously be addressed by an expert group-as should the relationship between adult and child doses for other organs. We understand that the authors of Draft WASH-1400 propose to revise their numbers in the final report. Their tentative revised number for the 1-9 year thyroid dose conversion factor is in the middle of the range between the published Draft WASH-1400 values and the EPA numbers (NRC, 1975a).

\section{c. External doses}

In addition to the inhaled radioisotopes, the gamma emissions from radioactive nuclei in the passing cloud and from those deposited on the ground would also contribute to the whole-body dose. The dose from "cloud shine" for an integrated exposure of 1 curie-sec $/ \mathrm{m}^{3}$ and the hourly dose rate from "ground shine" for a contamination level of 1 curie $/ \mathrm{m}^{2}$ are given in Draft WASH-1400, Appendix VI, page 60. They are reproduced in Table XXXVII. These doses have been calculated for $5 \mathrm{~cm}$ tissue depth, without additional shielding factors. The contribution of the cloud shine was calculated in the approximation that the cloud is very thick in comparison with the mean free path of the gammas (which is of the order of 100 meters).

For purposes of orientation, let us compare the doses from cloud and ground shine assuming a deposition velocity of 0.2 $\mathrm{cm} / \mathrm{sec}$. The relationship between the integrated exposure $\chi$ and the ground contamination $\sigma$ is given by

$$
\sigma=v_{d} \chi \text { curies } / \mathrm{m}^{2}
$$

or

$$
\sigma=2 \times 10^{-3} \chi \text { curies } / \mathrm{m}^{2} .
$$

From Table XXXVII, it will be seen that for this deposition velocity, it will take of the order of 5 to 10 hours of exposure for the whole-body dose from radionuclides deposited on the ground to equal that due to the corresponding cloud dose.

It is instructive to compare these doses with the whole-body inhalation doses which we have tabulated in Table XXV. The curies inhaled for an exposure of $\chi$ curies $-\mathrm{sec} / \mathrm{m}^{3}$ and an adult breathing rate of $230 \mathrm{cc} / \mathrm{sec}$ are

$$
\mathrm{I}=2.3 \times 10^{-4} \chi \text { curie }
$$

From Table XXXV (Doses Due to Inhalation), it will be seen that the whole-body dose due to inhalation will exceed that which had been received by external gamma radiation during the passage of the cloud-typically by an order of magnitude. If evacuation occurs reasonably rapidly, we therefore expect the large short-term radiation doses to be incurred dominantly via the inhalation route. At greater distances, where doses 
are lower and evacuation is not carried out, the integrated dose due to ground contamination can be the dominant wholebody dose.

\section{Dose effects}

\section{Immediate}

Calculations of immediate deaths, due to acute radiation damage, either internal or external, are very difficult to make. Acute lethal doses are in the ranges of hundreds (whole-body) to thousands (lung, GI-tract) of rem. We cannot use the wedge model to estimate acute effects because it is inapplicable at distances short compared with an atmospheric mixing length. As already noted, the number of doses in the lethal range will depend sensitively on plume rise, atmospheric stability, evacuation, shielding by houses, population density close to the site, and assumed biological threshold doses. We did not have the resources or time to do an independent calculation of the immediate deaths expected under various conditions. For this reason we concentrated on the cancer induction and genetic defects associated primarily with the lower doses experienced at great distances (which are, of course, in addition to the immediate fatalities, although they take place over decades rather than weeks).

However, we note that certain effects seem not to have been discussed adequately in the previous estimates of acute damage and these effects may make substantial differences to the results in this area:

We note that there is apparently great uncertainty as to the thresholds for acute biological effects resulting from the inhalation of radionuclides. The authors of Draft WASH-1400, for example, have modified the standard ICRP values for doses to the GI tract by introducing large shielding factors associated with the mucous coating and epithelial lining of the intestines (Appendix VI, p. 43). We have been unable to find any basis in the references given there for the large shielding factors invoked, but unlike experiments on radiation-induced cancer and genetic defects, experiments to determine the approximate thresholds for acute radiation effects due to the inhalation of an appropriate mixture of important radionuclides can be performed relatively rapidly using large mammals. Such experiments might substantially reduce the uncertainties involved in calculations of acute effects on humans. We urge that they be done. [Final WASH-1400 will clarify this issue. The authors assert that even without the assumed shielding, immediate deaths due to GI-tract irradiation are not larger than immediate deaths due to lung and whole-body irradiation (NRC, 1975a).]

\section{Cancer dose-response coefficients}

We will use as our guide in this discussion a standard reference for the dose-response coefficients, "The Effects on Populations of Exposures to Low Levels of Ionizing Radiation"

(NAS, 1972).

The numbers in this reference are intrinsically uncertain since they are based on a rather small number of well-established cases where human cancers were produced by radiation exposure. Because the statistics are small, the exposures uncertain, and the followup incomplete in these cases, it has been impossible to use them to explore, in any but the roughest manner, the dependence of the dose-response coefficient on age-at-exposure, sex, race, length-of-time after exposure, or dose rate. Also, most analyzed experiences have necessarily been associated with relatively high doses (hundreds of rem) because smaller effects associated with smaller doses would not have been detected.

For this reason, extrapolation is necessary in order to calculate the effects of the individual doses (of a few rems to tensof-rems, often delivered over a period of years) which would dominate the total population dose from a major reactor accident. In the absence of a basis for a verifiable extrapolation, it is ordinarily assumed that the relationship between dose and the probability of cancer induction is linear below the hundredsof-rem region where the data exist. Although some animal data indicate a nonlinear relationship, the NAS report concludes that:

"At this time, the linear hypothesis, which allows the mean tissue dose to be used as the appropriate measure of radiation exposure, provides the only workable approach to numerical estimation of the risk in a population. Further, since there are no means at present of determining the value of the dose-effect slope in the low dose region of interest, use of the linear extrapolation

TABLE XXXVII. Direct doses from the plume and from fallout.

\begin{tabular}{|c|c|c|c|}
\hline Is otope & $\begin{array}{l}\text { Half-life } \\
\text { days (yr) }\end{array}$ & $\begin{array}{c}\text { Ground dose } \\
\text { (rems/hr)/ } \\
\left(\text { curie } / \mathrm{m}^{2}\right)\end{array}$ & $\begin{array}{c}\text { Cloud dose } \\
(\text { rems } / \mathrm{hr}) / \\
\left(\text { curie sec } / \mathrm{m}^{3}\right)\end{array}$ \\
\hline${ }^{85} \mathrm{Kr}$ & $10.760(\mathrm{yr})$ & $\cdots$ & $\cdot 0$ \\
\hline${ }^{85} \mathrm{Kr}^{\mathrm{a}}$ & 0.180 & $\ldots$ & 0.036 \\
\hline${ }^{87} \mathrm{Kr}$ & 0.053 & $\cdots$ & 0.36 \\
\hline${ }^{88} \mathrm{Kr}$ & 0.116 & ... & 0.42 \\
\hline${ }^{89} \mathrm{Sr}$ & 50.6 & 0.0 & 0 \\
\hline${ }^{90} \mathrm{Sr}$ & 27.7 & 0 & 0 \\
\hline${ }^{91} \mathrm{Sr}$ & 0.4 & 28.4 & 0.16 \\
\hline${ }^{90} \mathrm{Y}$ & 2.7 & 0.02 & 0.002 \\
\hline${ }^{91} \mathrm{Y}$ & 59 & 0.02 & 0.002 \\
\hline${ }^{95} \mathrm{Zr}$ & 65.5 & 20.0 & 0.19 \\
\hline${ }^{97} \mathrm{Zr}$ & 0.7 & 23.8 & 0.06 \\
\hline${ }^{95} \mathrm{Nb}$ & 3.5 & 20.4 & 0.18 \\
\hline${ }^{99} \mathrm{Mo}$ & 2.8 & 7.2 & 0.06 \\
\hline${ }^{99} \mathrm{Tc}$ & 0.25 & 4.0 & 0.035 \\
\hline${ }^{103} \mathrm{Ru}$ & 40 & 14.4 & 0.11 \\
\hline${ }^{105} \mathrm{Ru}$ & 0.18 & 18.0 & 0.20 \\
\hline${ }^{106} \mathrm{Ru}$ & 1 & 6.0 & 0.05 \\
\hline${ }^{105} \mathrm{Rh}$ & 1.5 & 2.8 & 0.005 \\
\hline${ }^{129} \mathrm{Te}$ & 0.048 & 3.0 & 0.018 \\
\hline${ }^{129} \mathrm{Te}$ & 34.1 & 3.0 & 0.025 \\
\hline${ }^{131} \mathrm{Te}$ & 1.25 & 33.6 & 0.375 \\
\hline${ }^{132} \mathrm{Te}$ & 3.25 & 6.8 & 0.05 \\
\hline${ }^{131} \mathrm{I}$ & 8.05 & 11.2 & 0.09 \\
\hline${ }^{132} \mathrm{I}$ & 0.1 & 68.0 & 0.55 \\
\hline${ }^{133} \mathrm{I}$ & 0.875 & 14.8 & 0.12 \\
\hline${ }^{134} \mathrm{I}$ & 0.036 & 64.0 & 0.60 \\
\hline${ }^{135} \mathrm{I}$ & 0.28 & 48.0 & 0.42 \\
\hline${ }^{133} \mathrm{Xe}$ & 5.3 & $\ldots$ & 0.007 \\
\hline${ }^{135} \mathrm{Xe}$ & 0.38 & . & 0.06 \\
\hline${ }^{134} \mathrm{Cs}$ & $(y r)$ & 48.0 & 0.36 \\
\hline${ }^{136} \mathrm{Cs}$ & 12.9 & 60.0 & 0.46 \\
\hline${ }^{137} \mathrm{Cs}$ & 30 & 16.8 & 0.13 \\
\hline${ }^{140} \mathrm{Ba}$ & 12.8 & 8.4 & 0.06 \\
\hline${ }^{140} \mathrm{La}$ & 1.66 & 60.0 & 0.52 \\
\hline${ }^{141} \mathrm{Ce}$ & 32.8 & 2.2 & 0.016 \\
\hline${ }^{143} \mathrm{Ce}$ & 1.37 & 8.8 & 0.085 \\
\hline${ }^{144} \mathrm{Ce}$ & 285 & 1.2 & 0.004 \\
\hline${ }^{143} \mathrm{Pr}$ & 13.6 & 0.0 & 0 \\
\hline${ }^{147} \mathrm{Nd}$ & 11 & 4.0 & 0.045 \\
\hline${ }^{147} \mathrm{Pm}$ & $2.65 \quad(y r)$ & 0.0 & 0 \\
\hline${ }^{149} \mathrm{Pm}$ & 2.2 & 0.1 & 0.012 \\
\hline${ }^{238} \mathrm{Pu}$ & 86.4 & 0.004 & 0 \\
\hline${ }^{239} \mathrm{Pu}$ & 24390 & 0.004 & 0 \\
\hline
\end{tabular}

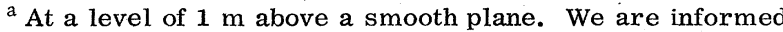
that the coefficients used in Draft WASH-1400 are low by a factor of 2 (NCR, 19756). Our values for the dose include this correction. 
from data obtained at high doses and dose rates may be justified on pragmatic grounds as a basis for risk estimation" (NAS, 1972, p. 89).

Draft WASH-1400 also uses the assumption that the probability of cancer or genetic abnormality in the population is linearly proportional to the man-rem exposure, independent of its distribution in the population (at subacute levels). Perhaps, when an improved theoretical understanding of the mechanisms of cancer induction is arrived at, it may be possible to devise a more sophisticated extrapolation procedure. The other basic document in the field (UNSCEAR, 1972, p. 431) states:

"The Committee wishes to reemphasize that all the estimates apply to short-term exposure at high-dose rates and, as discussed in Annex G, are likely to be over-estimates of the risk per unit dose that may result from protracted irradiation at low-dose rates and with low-LET radiation."

\section{Cancer from whole-body dose}

"Despite many uncertainties, an approximate estimate of over-all cancer mortality can be made on the basis of followup studies on Japanese atomic bomb survivors and patients treated with radiation for diseases other than cancer. In those populations, the excess mortality from all forms of cancer corresponds to roughly 50-165 deaths per $10^{6}$ persons per rem during the first $25-27$ years after irradiation" (NAS, 1972, p. 91).

There are several methods of extrapolating this range to the expected cancer induction rate for the U.S. population as a whole (i.e., a population with age distribution of the U.S.).

On the basis of an "absolute-risk model," taking into account latent periods, lifetime risk, and increased sensitivity to radiation at certain stages, we can estimate for the U.S. population an excess mortality due to cancer of about 130 per million man-rem.

Certain sensitive organs, in particular lungs and thyroid, receive large beta doses. This contribution to the whole-body dose is small because of the short range of the betas. Since lung cancers comprise about $20 \%$ of the cancers due to wholebody dose from external radiation, there may be many more from the localized beta dose, and these should be estimated separately.

\section{Lung cancer from lung dose}

"For cancer of the lung, the mortality at high doses has been observed to approximate one death per $10^{6}$ exposed persons per year, per rem." (NAS, 1972, p. 87.) The results range from 0.6 to 1.61 , and the years in question range from 7 to 50 years after exposure (typically only an average of ten years per subject in the sample, however). (NAS, 1972, pp. 150-151.)

\section{Thyroid cancer from thyroid dose}

Iodine is readily absorbed into the body following inhalation and is concentrated to a large degree in the relatively small thyroid gland. (See Table XXXVI.) The thyroid is also quite susceptible to radiation-induced tumors-especially for persons exposed as children. These two reasons account for the fact that, of the 19 Marshallese children under the age of 10 on Rongelap Island who were accidentally exposed to fallout from a nuclear test on Eniwetok Island in 1954, all but two suffered thyroid damage: 14 cases of benign tumors, one malignant thyroid cancer, 2 thyroid atrophies with consequent stunted growth (Conard, 1975).

The best statistics for the dose-effect relationship for thyroid cancer come from the Marshallese data (as analyzed by R. Con- rad) and from followup investigations (by L. Hempelman and collaborators at the University of Rochester) on a group of persons subjected to large $\mathrm{x}$-ray doses to the thymus gland (and nearby thyroid) in infancy. For the incidence of benign thyroid tumors in persons exposed to $x$-rays before age ten, we will use Hempelman's number: " 38 to 52 cases per $10^{6}$ persons exposed per rad thyroid dose per year" (Hempelman, 1968). Approximately one-third of these benign tumors were treated surgically. In Hempelman's latest survey (Hempelman et al ., 1975), the reported increased incidence of both benign and malignant thyroid tumors has been found to continue more than 30 years after exposure. The latest reviews of the Rongelap children (we defined children here as persons exposed before the age of ten) do not change the nodule data for children much from the data in the summary by Hempelman in his 1968 review paper. By 1966, all but two of these nineteen children had developed tumors or had had their thyroids atrophy. The fifteen who developed tumors were operated on, and an attempt has been made to keep the entire group on medication since 1965 (Conrad, 1975).

Regarding the incidence of nonfatal thyroid tumors in persons exposed after age 9, we have only Conard's data. Taking his full Marshall Island sample, the incidence of benign nodules among persons exposed after the age of 9 seems somewhat higher than among the unexposed population-but the effect is much less significant than for the persons exposed at higher doses as children, and we shall not attempt to extract a number.

As regards malignant thyroid tumors, the NAS report estimates a risk of 1.6 to 9.3 cases per year per million childhood rem averaged over 25 to 30 years following exposure (NAS, 1972 , pp. 120-125). The upper end of the range is derived from Hempelman's data. In Hempelman's latest survey, using a new and more plausible averaging procedure, he obtains an average value for the corresponding coefficient (with an assumed latent period of 5 years) of 3.0. The Marshallese data, based on one carcinoma, are consistent with this number. We take the best value for the coefficient now to be $1.6-3.0$ per year per million man-rem.

Finally, we come to thyroid cancer in persons exposed after age 9. Here we have the Nagasaki, Hiroshima, and Marshall Island data. The Hiroshima-Nagasaki data appear to show an elevated incidence of thyroid cancer for women exposed as adults-perhaps one-half of the excess rate per rem for women exposed as children-but the statistics are marginal (UNSCEAR, 1972, p. 437).

Conrad reports two carcinomas (identified in 1965 and 1967, respectively) among his sample of 34 persons on Rongelap exposed after age 9 to an average thyroid dose of $379 \mathrm{rad}$. Conard obtains from this group an incidence of about 6 per million thyroid rad per year averaged over 20 years. If one averages only the years before medication began in 1965, the incidence would approximately double and subtraction of a latent period of 5 years might lead to yet another doubling. And, of course, with 2 cases, the average incidence might well be half or twice as large as estimated. Such calculations are a large burden to put on such small statistics so we will conclude only that the combined message of the Hiroshima-Nagasaki and Rongelap data is to suggest that, per rem, the likelihood of thyroid cancer induction in adults is of the same order of magnitude as that in persons exposed before age 10 .

One key question is whether a rem from radioiodine $\left({ }^{131} \mathrm{I}\right.$ in particular) will be as effective in producing thyroid tumors as a rem from x-rays. The NAS report gives a reference to an experiment with rats where the carcinogenic effect of ${ }^{131}$ I at very high doses appears to be approximately one-tenth that of $x$ rays (Doniach, 1963). Russian workers seem to have found similar effects and attribute them to the fact that the most sensitive cells in the thyroid receive much less than the average thyroid dose due to the uneven distribution of the iodine in the thyroid and the short range of the beta-rays emitted by ${ }^{131} \mathrm{I}$. 
The other radioiodines emit more energetic beta-rays, however, and the Russians find their radiation at least as effective biologically as x rays, rem-for-rem (Klassovskii, 1967, pp. 40-42; Ilin et al ., 1972, p.48).

Since slightly more than one-quarter of the population thyroid dose calculated in Draft WASH-1400 is due to these other radioiodines (see below), the average biological effectiveness of the iodine dose would, according to these results, be about onethird that of $\mathrm{x}$-rays. (The dose to the Rongelap group thyroids was dominated by short-lived radioiodines so that the consequences for this group do not contradict the possibility of a lower relative effectiveness of ${ }^{131} I_{0}$ ) The NAS report notes that the evidence for humans is quite complex and the report does not draw any conclusion on the relative effectiveness of the radioiodines and $\mathrm{x}$-rays in humans.

In view of this situation, we have decided to assign an uncertainty of a factor in the range from $0.3-$ to- 1 to the effectiveness of the radioiodines in the production of thyroid nodules and thyroid cancers, relative to the corresponding effectiveness of $\mathrm{x}$-rays per rem.

The mortality rate from radiation-induced thyroid cancer seems to be quite low, but the mortality rate from spontaneous thyroid cancer for persons less than 45 also seems to be quite low-96\% 5-year relative survival rate (NCI, 1972, p. 164)。 The best-studied populations, the Rochester group which received thymus x-ray irradiation as children, and the Marshallese children who were exposed to fallout in 1954, are still typically in their $30 \mathrm{~s}$ and early $40 \mathrm{~s}$. Beyond age 45 , the mortality for "spontaneous" thyroid cancer not attributable to radiation increases, and the age-averaged 5-year relative survival rate is reduced to $84 \%$ (NCI, 1972, p. 162). (Data for patients diagnosed during the later period 1965-1969 are also given in the same reference-but not broken down by age and with no five-year relative survival rate. The three-year relative survival rate has improved, however-from $85 \%$ to $88 \%$.)

\section{Genetic defect dose-effect coefficients}

"We take the risk of chronic radiation at low doses relative to the spontaneous mutation rate to be 0.005 to 0.05 per rem. The information on the radiation-induced effect comes almost entirely from mouse data." (NAS, 1972, p. 59.) "The current incidence of new known dominant genetic defects is estimated at about 2,000 per million live births" (NAS, 1972, p. 54).

We will assume that the genetically effective man-rem are equal to half the whole-body dose, since only the radiation received by individuals prior to procreation will be effective. The increased number of added genetic defects per million whole-body man-rem (scaled from mouse-rem) will, therefore, be 5 to 50. Each of these dominant genetic defects is assumed to have an average persistence of five generations (NAS, p. 23). Multiplying by this factor would give 25 to 250 additional genetically defective persons per million whole-body man-rem. (This may overstate the perceived costs, since a defect expressed in the fourth generation might be discounted substantially in present value.) To this must be added 12.5 noninheritable congenital anomalies and 42 spontaneous abortions (NAS, 1972 , p. 55). The NAS report also speculated concerning the possible genetic component of constitutional and degenerative diseases and general ill health, expressing concern that "there is danger that the previous sections, by concentrating only on fairly-well-defined genetically-associated diseases, have dealt with only the exposed part of the iceberg" (NAS, p. 56). An estimate of 0-50 additional constitutional and degenerative diseases is offered, per million.whole-body man-rem with an average persistence of 10 generations, hence $0-500$ total extra constitutionally or degeneratively diseased persons (an average 100 years hence), (NAS, 1972, p. 57).

In Table XXXVIII we summarize the numbers discussed above for the man-rem effects.

\section{Land and property contamination}

A reactor accident on the scale of the reference accident would result in contamination of land and property. Public authorities would have the problem of setting action thresholds for the evacuation (and later the return) of populations, for condemnation of crops and milk, for decontamination efforts, etc. There are several pathways by which radiation can affect the population:

- direct gammas from radionuclides which have plated out on the ground and onto or inside buildings;

-resuspension and inhalation of radioactive material;

- ingestion of agricultural products produced on contaminated land;

-consumption of contaminated water.

We discuss some of the more important pathways below.

It will turn out (Table XLI) that iodine is the dominant source of direct radiation initially following an accident. To calculate the dose over longer periods, we need to know the effective half-life of the radionuclides, as natural attrition rates may be greater than the radioactive decay rate of the long-lived isotopes of concern here. Over the long term, cesium dominates the external gamma dose. We describe this calculation in some detail, as we regard it as an important omission from Draft WASH-1400.

Draft WASH-1400 suggests taking as an evacuation threshold an integrated whole-body dose of the order of $10 \mathrm{rem}$ over the first year of exposure. The corresponding contamination levels for agricultural land and property are given (Draft WASH-1400,

TABLE XXXVIII. Some man-rem dose-effect coefficients for delayed effects (per million person rem).

A. Whole body effects

About 130

$25-250$

Cancer deaths nant genetic defects over an average of five generations following exposure

12.5

$0-500$ Noninheritable congenital defects Total extra constitutionally or degeneratively diseased persons over an average of ten generations following exposure 42 Spontaneous abortions

B. Lung dose effects $0.6-1.6$

Cancer deaths per year for the period 5 to at least 27 years following exposure

C. Thyroid dose effects Children under 10 $0.5^{\mathrm{a}}-3.0$

Cancer cases per year from 5 years till at least 30 years following exposure. (times about 0.04 for mortality during this period)

$11^{a}-52$ Cases of thyroid nodules per year from 5 years till at least 30 years following exposure

Adults (persons over 10) $0.5^{\mathrm{a}}-3.0$

Cancer cases per year from 5 years till at least 25 years following exposure. (times about 0.15 for mortality)

${ }^{a}$ (The lower value would hold if ${ }^{131}$ I were rem-for-rem 0.3 times as effective in producing thyroid cancer than $x$ rays.) 
Appendix VI, p. 66) as shown in Table XXXIX. Of course, the "allowable dose" might be saturated by any one of these isotopes at its threshold level-if two or more are important, the thresholds for particular isotopes may be reduced.

In order to estimate damage from this contamination, we must know the duration of exposure. There is some evidence on the persistence of ${ }^{90} \mathrm{Sr}$ and ${ }^{137} \mathrm{Cs}$ in soil from the study of fallout from atmospheric nuclear tests (UNSCEAR, 1972). For ${ }^{90} \mathrm{Sr}$ availability to crops, the natural half-life in soil is quoted as 5 to 7 years (p. 45). For ${ }^{137} \mathrm{Cs}$ it is stated that "The transfer from deposit to diet is normally characterized by high uptake during the first years after deposition and by relatively small uptake subsequently" (p. 52). External radiation from ${ }^{137} \mathrm{Cs}$ is of concern also, however, because of the associated gamma emission (the average gamma energy is $0.66 \mathrm{MeV} ;{ }^{90} \mathrm{Sr}$ emits only betas upon decay). For the purposes of this external exposure the U. N. report quotes an empirically determined twocomponent dose rate:

$$
D(t)=0.12[0.63 \exp (-1.15 t)+0.37 \exp (-0.03 t)]
$$

$(\mathrm{rad}$ in air $/$ year $) /\left(\mu\right.$ curies $\left./ \mathrm{m}^{2}\right), t$ in years, (p. 55). (AII.23)

At time $t=0$ this dose rate of $0.12 \mathrm{rad}$ per year compares with the value derivable from Table XXXVII $(0.148 \mathrm{rem} / \mathrm{year}$, which, however, is taken at $5 \mathrm{~cm}$ tissue depth). The time constant in the first term is due to the movement of the ${ }^{137} \mathrm{Cs}$ deeper into the soil with the result that the gammas are somewhat attenuated. The second time constant, however, 'corresponds roughly to the natural decay half-life of ${ }^{137}$ Cs. Equation AII.23 indicates, therefore, that leaching into the soil will reduce the dose rate by a factor of three after two years or so, but that at least some of the ${ }^{137} \mathrm{Cs}$ becomes fixed in the soil and its residence half-life becomes comparable with its decay half-life. Equation AII.23 integrates to an infinite-time dose in air of $1.55 \mathrm{rad}$ per microcurie per square meter.

If we take the contamination threshold level for ${ }^{137} \mathrm{Cs}$ at which evacuation is made to be $100 \mathrm{microcurie} / \mathrm{m}^{2}$ as given in Table XXXIX, then the dose rate in air will be initially $12 \mathrm{rad} / \mathrm{year}$ and the integrated dose will be approximately $155 \mathrm{rad}$. This dose will be reduced by the shielding effects of ground roughness and buildings and by the fact that in built-up areas much of the contamination might be washed away. On the other hand, in built-up areas, the portion of the aerosol not washed away will also not be shielded by a layer of soil. Small aerosol particles can become quite strongly bound to surfaces by van der Waals forces. [J. W. Healy of Los Alamos in an unpublished paper "Nuclear Reactor Accident-Damage Assessment, Draft" dated 1966, (quoted in Draft WASH-1400, Appendix VI, as Ref. 41) cites a decontamination factor of only 0.7 for the removal of fallout from a sea detonation of a nuclear weapon by the firehosing of an asphalt road in Naval Research Laboratory tests.] Aerosol particles adhering to walls, roofs, and internal surfaces could also result in "antishielding" effects for the occupants relative to radiation from a uniform deposition on a flat open area. On the other hand, since Cs salts are highly soluble, rain will leach some cesium from exposed surfaces. A detailed analysis supported by experiments is obviously called for in order to obtain a better estimate. The U. N. report assumes that the average shielding factor for whole-body dose from ground contamination by ${ }^{137} \mathrm{Cs}$ is 0.4 , and for dose to the gonads 0.32 . We shall, therefore, use the same shielding factor as Draft WASH-1400 (Appendix VI, p. 58)-0.33, so that the integrated shielded dose from ${ }^{13^{\prime \prime}} \mathrm{Cs}$ is $0.52 \mathrm{rad}$ per microcurie $/ \mathrm{m}^{2}$.

For ${ }^{90} \mathrm{Sr}$, the integrated dose from the atmospheric testing of nuclear weapons via the milk pathway (associated with population-weighted fallout of $0.0584 \mathrm{microcurie} / \mathrm{m}^{2}$ ) has been computed as $0.045 \mathrm{rad}$ to the bone marrow in the northern hemisphere (UNSCEAR, 1972, p. 92). For a population consuming milk from an area contaminated with ${ }^{90} \mathrm{Sr}$ up to the maximum value assumed allowable (before diversion of supply) in Draft WASH-1400, 2 microcurie $/ \mathrm{m}^{2}$, the corresponding integrated dose to the bone marrow would be about 2.6 rad.
TABLE XXXIX. Land contamination thresholds.

\begin{tabular}{cccc}
\hline \hline Isotope & Half-life & $\begin{array}{c}\text { Use for human } \\
\text { occupation } \\
\left.\text { (micro curie } / \mathrm{m}^{2}\right)\end{array}$ & $\begin{array}{c}\text { Use for } \\
\text { agriculture } \\
\left.\text { (micro curie } / \mathrm{m}^{2}\right)\end{array}$ \\
\hline${ }^{89} \mathrm{Sr}$ & 0.14 & 50 & 10 \\
${ }^{90} \mathrm{Sr}$ & 27.7 & 20 & 2 \\
${ }^{131} \mathrm{I}$ & 0.022 & 1000 & 5 \\
${ }^{134} \mathrm{Cs}$ & 2 & 20 & 10 \\
${ }^{136} \mathrm{Cs}$ & 0.035 & 200 & 100 \\
${ }^{137} \mathrm{Cs}$ & 30 & 100 & 20 \\
${ }^{144} \mathrm{Ce}$ & 0.78 & 500 & 100 \\
${ }^{238} \mathrm{Pu}$ & 86.4 & 0.5 & 0.2 \\
${ }^{239} \mathrm{Pu}$ & 24390 & 0.2 & 0.1 \\
\hline \hline
\end{tabular}

\section{E. Consequence calculations}

\section{Introduction}

Calculations of the potential consequencies of a major nuclear reactor accident have been undertaken by the AEC on three different occasions, in 1957, 1965, and 1974.

\section{a. 1957 study}

In 1957, the AEC released a study entitled Theoretical Possibilities and Consequences of Major Accidents in Large Nuclear Plants (AEC, WASH-740, 1957). A 500 MWt reactor was considered. The accident was assumed to occur 180 days after the reactor was loaded with fresh fuel. Two different population distributions were assumed, along with various meteorological conditions, three different degrees of fission product release, two different temperatures for the released plume, and two different particle sizes for the released aerosol. Only immediate health effects were considered-i.e., not cancer or genetic effects. A very large range of consequences was calculated-ranging from zero effects on health for even the largest release (if the plume is assumed to rise thousands of feet off the ground due to heat buoyancy effects) to 3400 deaths and 43,000 injuries (for the release of $50 \%$ of the fission product inventory in the form of an aerosol with average diameter of one micron in a cold ground-level plume released under inversion conditions.) Similarly, the property damage ranged from negligible to a long-term (months or more) evacuation of 760 square miles and restrictions on agriculture over an area of 150,000 square miles.

\section{b. 1965 study}

Many of the assumptions made in the 1957 study were very rough. In 1965 an effort was, therefore, mounted to improve it. After drafts of the new report had been circulated for review within the AEC and the industry, however, the decision was made to abort the study. In 1973 the draft became publicly available as a result of a freedom-of-information suit. The draft report concluded that: "In the highly unlikely event of a major reactor accident, we have found no reason to believe that the extent of radiation damages would be any less than those estimated in WASH-740; conceivably the damages could be substantially greater" (AEC, WASH-740 update file, document 113 , p. 5). Reasons cited for the increase in the upper limit of the damage spectrum were increased power levels and fuel life in reactors, more restrictive personnel-exposure and contamination criteria, and the expected trend toward reactor siting in metropolitan areas. An effect working in the opposite direction (i.e., toward lower consequences) was data on fission-product release which indicated that the $50 \%$ release model in the 1957 study was unrealistically high. 
The authors of the 1965 study draft drew attention to the basic dilemma which faced them in conducting the risk assessment for nuclear reactors:

"Operational reactor safeguards would reduce the radiation damages resulting from accidents of all types and especially of the most catastrophic ones. However, from an analysis of safeguards now available, it cannot be assumed that these safeguards would be 100 percent effective in every circumstance.

"It should be pointed out that analysis of a major accident involving the juxtaposition of the most pessimistic values of every one of the numerous parameters leads to enormous potential damages. This amounts to taking full account of developments which tend to increase potential damages while discounting safeguards completely. Estimates of damages arrived at in this manner, without regard for improbability, must inevitably be unrealistic, but the only course available for a consistent study (AEC, WASH-740 update file, document 113, pp. 5-6).

\section{c. 1974 study}

In 1974 the AEC issued a draft report, "Reactor Safety Study: An Assessment of Accident Risks in U. S. Commerical Nuclear Power Plants," (Draft WASH-1400) which for the first time attempts to calculate the probabilities of accidents as well as their consequences. It concludes that the probabilities of the largest accident consequences calculated in WASH-740 are exceedingly small. The probability of a core meltdown is calculated to be of the order of $10^{-4}$ per reactor year (Appendix VI, p. 9) but it is calculated that in only one in 100,000 of these meltdown accidents (so $10^{-9}$ per reactor year) does the number of immediate deaths approach 3,000. Long-term evacuation of more than 100 square miles is calculated as necessary only once in every 30 meltdowns.

In this section we will not discuss the probabilities calculated in Draft WASH-1400, but we will discuss the assumptions on which some of the consequences have been calculated. We will concentrate in particular on the man-rem effects (i.e., cancer and genetic defects) and land-contamination effects, since they are less sensitive to details of the meteorological conditions and close-in population densities than are immediate deaths.

\section{Man-rem doses-comparison with wedge model calculations}

For specificity we consider the fission-product release characterized in Draft WASH-1400 as the "Reference Accident." (See Table XL.) 'This release (also denoted as PWR2) is assumed to result from an accident at the large PWR reactor described in Table XXXIII above and is described in Draft WASH1400 (Appendix VI, p. 10) as follows:

This category includes failure of core cooling systems, and core melting concurrent with a loss of containment spray and heat removal systems. Failure of the con-

TABLE XL. Fission product releases in Draft WASH-1400 reference accident (Appendix VI, p. 9).

Fractional release to atmosphere

\begin{tabular}{ll} 
Principal chemical groups & $\begin{array}{c}\text { from core } \\
\text { Noble gases }\end{array}$ \\
\hline Iodines & 0.9 \\
Cesiums & 0.7 \\
Telluriums & 0.5 \\
Alkaline earths & 0.3 \\
Volatile oxides & 0.06 \\
Nonvolatile oxides & 0.02 \\
\hline
\end{tabular}

tainment barrier occurs through over-pressure causing a substantial fraction of the containment atmosphere to be released in a "puff" from containment.

This release is estimated in Draft WASH-1400 to occur with an average probability of about six percent in all PWR meltdown accidents (Appendix VI, p. 9). The quoted uncertainty on the absolute probability is a factor of 10 (Summary, p. 124), and it is argued that upgraded maintenance procedures could reduce the average probability by a factor of five (Appendix V, pp。76-78). This release category is exceeded in severity only by the release associated with a steam explosion which causes the containment to fail.

We have used our simplified wedge model to make the same man-rem calculation as Draft WASH-1400 for the reference accident release using the parameters from Draft WASH-1400 given in Table XLI. The radial integral cutoff, the shielding factor, the release time, and the deposition velocities are identical to the values stated in Draft WASH-1400, Appendix VI; the other parameters represent weighted averages of the corresponding parameters used there. (See Draft WASH-1400, pp. 15-21.) In calculating the whole-body dose, the authors of Draft WASH-1400 make an additional approximation of truncating the time integral for the gamma dose from ground contamination at one day. We shall see that this results in the neglect of an important fraction of the long-term damage to health and life.

The whole-body doses obtained from the wedge model calculation with these approximations are shown in Table XLII and compared with the available Draft WASH-1400 results. It will be seen that the population doses computed from the wedge model are in good correspondence with the corrected Draft WASH-1400 results (except that Draft WASH-1400 does not calculate the long-term ground dose). ${ }^{28}$

Draft WASH-1400 also gives the contributions to whole-body dose of various elements (Appendix VI, p. 81). We list these values also in Table XLII along with the corresponding results obtained from the wedge model. It will be seen that the agreement with the corrected Draft WASH-1400 numbers is again quite reasonable. The only important discrepancy occurs in the case of iodine which also dominates the short-term ground doses calculated from the wedge model. (This may be due to a poorer approximation to Gaussian plume models by the wedge model for the uniquely high deposition velocity assigned to iodine in Draft WASH-1400.)

One additional comparison which is possible is that between the wedge model result and the Draft WASH-1400 result in the case of the average value of the 30-day lung population dose for PWR2. Again, the results are shown in Table XLII and the agreement is quite reasonable.

In Draft WASH-1400, partial evacuation is assumed within a 20 -mile radius of the reactor. This has been ignored in the wedge model. In order to test the sensitivity of the wedge model to such an evacuation, we have overstated the effects of evacuation by setting the population to zero within the $20-$ mile radius. Overall, total evacuation within 20 miles reduces the whole-body dose computed from the wedge model by $8 \%$ (in comparison to 6 percent in the Draft WASH-1400 calculation, Appendix VI, p. 81).

\section{Sensitivity to changes in assumptions}

The above results establish the wedge model as a useful-although not perfect-device for testing the sensitivity of the

\footnotetext{
${ }^{28}$ It should be noted, however, that the population density assumed in Draft WASH-1400 averages 165 per square mile (NRC, 1975a), while our detailed calculations are for 300 per square mile. See Footnote 15 (Sec. V.I.1) for a more extensive discussion of this point.
} 
TABLE XLI. Wedge model calculation of average Draft WASH-1400 reference accident population dose assumptions.

\begin{tabular}{ll}
\hline \hline & \\
Wind speed $(u)$ & $3.5 \mathrm{~m} / \mathrm{sec}$ \\
Inversion height $(H)$ & $1100 \mathrm{~m}$ \\
Plume angular width $(\theta)$ & $0.25 \mathrm{radians}$ \\
Deposition velocities $\left(v_{d}\right)$ & $\mathrm{cm} / \mathrm{sec}$ \\
$\quad$ Iodines & 0.5 \\
$\quad$ Noble gases & 0.0 \\
$\quad$ Other isotopes & 0.2 \\
Uniform population density & $300 \mathrm{mi}^{-2}$ \\
Cutoff on radial integrals & $500 \mathrm{miles}^{-2}$ \\
Shielding factor for ground and & $\frac{1}{3}$ \\
$\quad$ cloud dose & \\
Time between shut down of fission & 2.5 hours $^{\mathrm{a}}$ \\
$\quad$ and release &
\end{tabular}

A. Population doses from inhalation

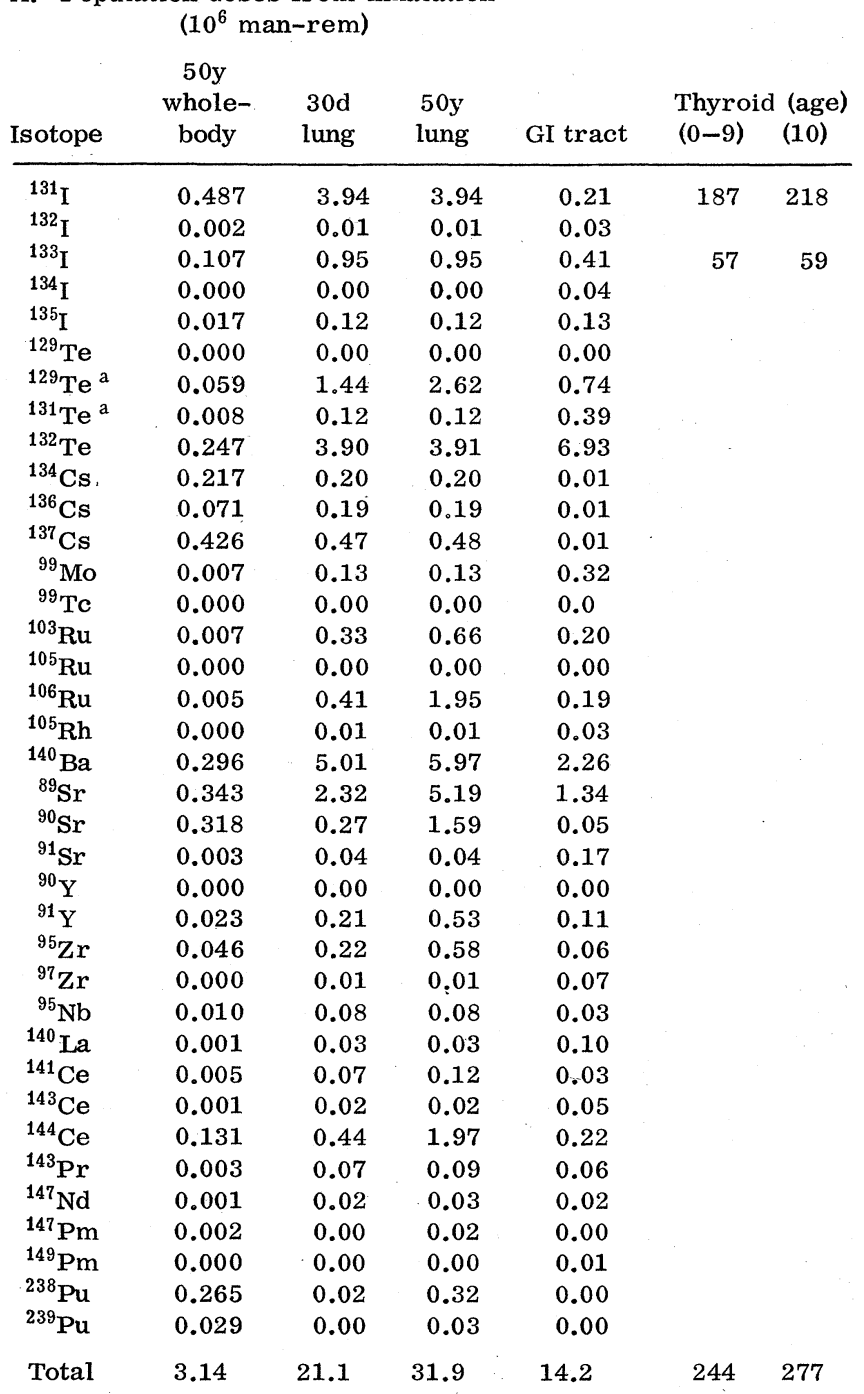

B. External gamma doses

\begin{tabular}{llll} 
& & \multicolumn{2}{c}{ Ground dose } \\
\cline { 3 - 4 } Isotope & Cloud dose & $(1$ day $)$ & Total \\
\hline${ }^{131} \mathrm{I}$ & 0.025 & 0.35 & 4.24
\end{tabular}

TABLE XLI. (Continued)

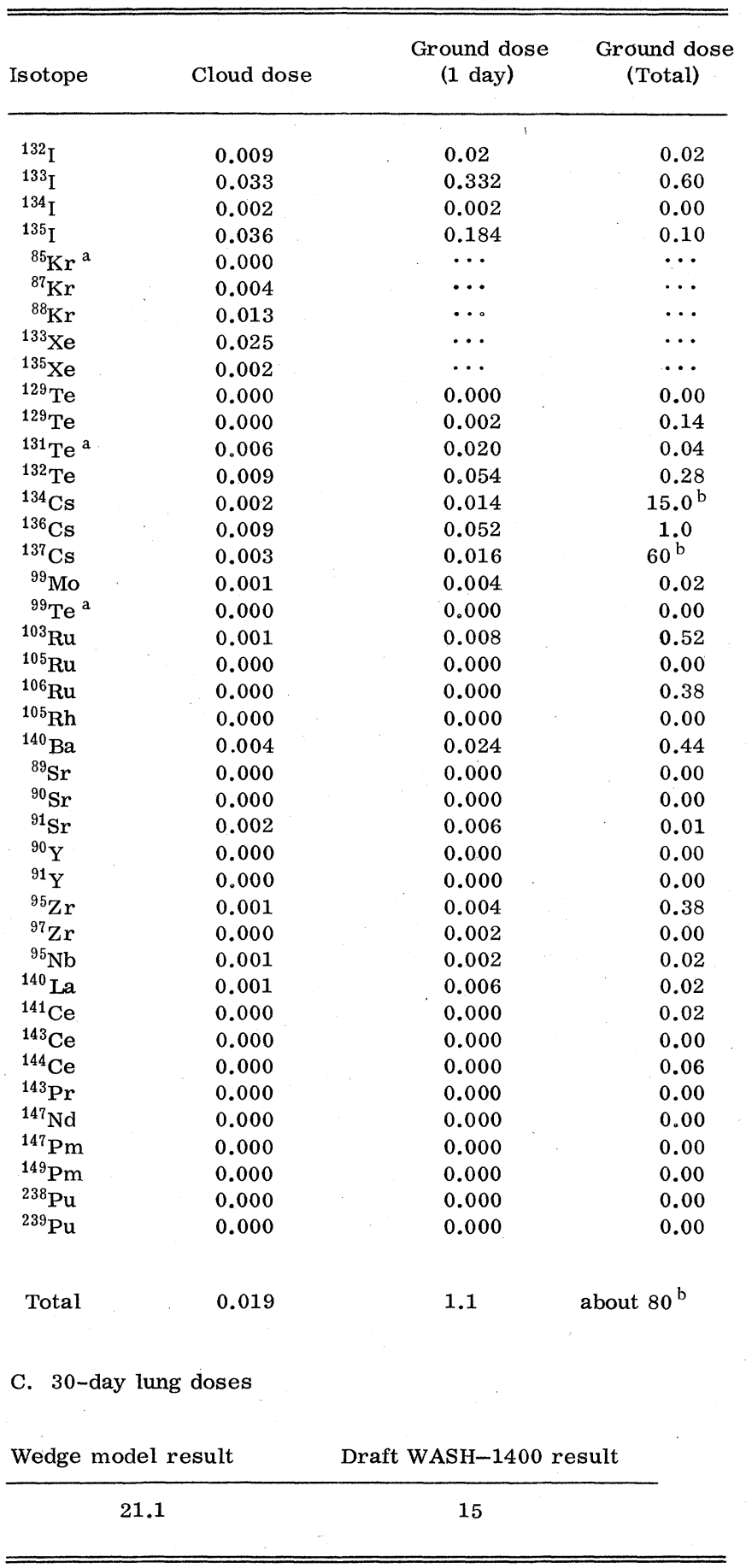

${ }^{a}$ But see (NRC, 1975b)

b The ${ }^{137} \mathrm{Cs}$ dose is calculated with an attrition as represented for ${ }^{137} \mathrm{Cs}$ by Eq. AII-23 and with that initial dose rate per microcurie $/ \mathrm{m}^{2}$ for ${ }^{137} \mathrm{Cs}$. ${ }^{134} \mathrm{Cs}$ dose uses the same attrition (accelerated by the 2-year half-life) but the initial dose rate is taken as $0.36 \mathrm{rad} / \mathrm{yr}$. per microcurie $/ \mathrm{m}^{2}$, in view of the total $1.58 \mathrm{MeV}$ gamma ray energy vs. the $0.66 \mathrm{MeV}$ gamma ray for ${ }^{137} \mathrm{Cs}$. 
TABLE XLII. Comparison of Draft WASH-1400 reference accident population doses with corresponding wedge model results (millions of man-rem).

A. Total whole body doses

Wedge model

Draft WASH-1400

\begin{tabular}{llll} 
& Wedge model & Draft WASH-1400 \\
\hline Inhalation dose & 3.1 & 2.9 & $2.9^{\mathrm{a}}$ \\
Cloud dose & 0.19 & 0.09 & $0.16^{\mathrm{a}}$ \\
1 day ground dose & 1.1 & $0.13 \quad 0.46^{\mathrm{a}}$ \\
Long-term ground dose & 80.0 & (Not calculated) \\
\hline
\end{tabular}

B. Contributions to whole body doses from particular elements

\begin{tabular}{|c|c|c|c|c|c|c|}
\hline \multicolumn{5}{|c|}{ Wedge model results } & \multicolumn{2}{|c|}{ Draft WASH-1400 results } \\
\hline $\begin{array}{c}\text { Element } \\
\text { (classes) }\end{array}$ & Inhalation & $\begin{array}{l}\text { Ground } \\
\text { (1 day) }\end{array}$ & Cloud & Total & \multicolumn{2}{|c|}{ Total } \\
\hline $\mathrm{Kr}$ & $\cdots$ & $\cdots$ & 0.017 & 0.017 & 0 & $0^{\mathrm{a}}$ \\
\hline $\mathrm{Sr}+\mathrm{Ba}$ & 0.96 & 0.03 & 0.006 & 0.996 & $0.85^{b}$ & $1.1^{\mathrm{a}}$ \\
\hline$R u+M o+T c+R h$ & 0.019 & 0.012 & 0.002 & 0.033 & $0.14^{\mathrm{c}}$ & $0.03^{a}$ \\
\hline $\mathrm{Te}$ & 0.314 & 0.076 & 0.015 & 0.405 & 0.22 & $0.31^{\mathrm{a}}$ \\
\hline I & 0.613 & 0.888 & 0.08 & 1.581 & 0.33 & $0.82^{\mathrm{a}}$ \\
\hline $\mathrm{Xe}$ & $\ldots$ & $\cdots$ & 0.027 & 0.027 & 0.01 & $0^{\mathrm{a}}$ \\
\hline $\mathrm{Cs}$ & 0.715 & 0.082 & 0.014 & 0.811 & 0.65 & $0.7^{\mathrm{a}}$ \\
\hline $\mathrm{Ce}$ & 0.137 & 0.000 & 0.000 & 0.137 & 0.12 & $0.12^{\mathrm{a}}$ \\
\hline $\mathrm{Pu}$ & 0.294 & 0.000 & 0.000 & 0.294 & 0.26 & $0.26^{\mathrm{a}}$ \\
\hline
\end{tabular}

${ }^{a}$ Corrected for programming and other errors (NRC, 1975b).

${ }^{b}$ Listed in Draft WASH-1400 as dose for Sr alone.

${ }^{c}$ Listed in Draft WASH-1400 as dose for Ru alone.

Draft WASH-1400 results to the variation of different assumptions. We consider the effects of these variations below:

\section{a. 500-mile cutoff}

Integrating the whole-body population dose to infinity rather than merely to 500 miles increases it by approximately a factor of 2.1 (still assuming average population density of $300 \mathrm{mi}^{-2}$ ). (The corresponding factors for cloud and long-term ground doses are 1.3 and 3 , respectively. The factors for the GI tract, thyroid, and long-term lung doses are $1.3,2$, and 2.3 , respectively.) This is not consistent with the statement in Draft WASH-1400 (Appendix VI, p.53) that "An analysis showed that there was only a very small contribution to the man-rem doses beyond this distance."

This result does not necessarily mean that the Draft WASH1400 results should be doubled for this reason. For most current reactor sites it appears to us quite probable that beyond 500 miles the plume would be over a region of population density which is low in comparison to that assumed here $\left(300 / \mathrm{mi}^{2}\right)$ or over the ocean. On the other hand, these results do indicate that it may be quite difficult to site a reactor so remotely as to reduce the average expected population dose from a major release of radioactivity by as much as an order of magnitude.

\section{b. Higher release fractions}

Meltdown release fractions for the volatile and nonvolatile oxides much higher than those assumed for the reference accident are compatible with the experiments cited in Draft WASH1400 (Appendix D of Appendix VII). To determine the sensitivity of the consequences to those assumptions, we have considered a case for which the release fractions of the volatile and nonvolatile oxides increase to $50 \%$ and $2 \%$, respectively, from the values given in Table XL. This is probably near the upper limit of the range allowed by the experimental data. The result is to increase the Draft WASH-1400 whole-body dose by a factor of 1.8 , the long-term ground dose by a factor of 1.1 , the 30-day lung dose by a factor of 2.2 , the long-term lung dose by a factor of 3.5 , and the GI-tract dose by a factor of 2.5 .

\section{c. Truncation of time integra/s}

In the absence of contrary evidence, the probability of cancer induction is ordinarily assumed to be proportional to the total integrated radiation dose. The long-term integrated dose was calculated in Draft WASH-1400 for the whole-body inhalation dose and for the thyroid, GI-tract, and cloud doses (which by their nature are given over relatively short periods)-but not for the lung doses and ground doses.

It may be seen from Table XLI that the long-term lung dose is in the wedge model increased by approximately a factor of 1.5 relative to the 30 -day dose. For the ground dose (relative to the one-day dose) the effect is much larger-by a factor of 70 .

Draft WASH-1400 integrates the ground dose only for the first day because "people will be evacuated from highly radioactive areas" (Appendix VI, p. 58). Elsewhere the assumed threshold for evacuation action in the case of contamination by ${ }^{137} \mathrm{Cs}$ is taken to be at a contamination level of $100 \mathrm{microcurie} / \mathrm{m}^{2}$ (see Table XXXIX). In the wedge-model approximation, the average level of land contamination will fall below this threshold value at a distance of approximately 37 miles. This corresponds to an average contaminated land area in the sector within this radius of $170 \mathrm{mi}^{2}$. The corresponding figure is not given in Draft WASH-1400, but, in Appendix VI on p. 79, the average land area contaminated at the probability level assigned to the PWR2 release $\left(5 \times 10^{-6}\right.$ per reactor year $)$ is shown as about $30 \mathrm{mi}^{2}$. At a probability of $10^{-5}$ the area is shown as about $3 \mathrm{mi}^{2}$ and at a probability of $10^{-6}$ per reactor year, the contaminated area shown as $200 \mathrm{mi}^{2}$. In all these cases (with a plume width of 0.25 radians) the area contaminated above evacuation threshold has a radius of less than 40 miles. Within this limited area the long-term integrated population ground dose-as calculated by the wedge model-accounts for less than 10 percent of the total population dose integrated out to 500 miles. Thus evacuation of the regions deemed "highly radioactive" in Draft WASH-1400 does not prevent most of the long-term population exposure.

It is possible that one might evacuate out to a greater distance, but a rather delicate cost-benefit calculation would be involved, in which the benefit would be the elimination of the 
small increased individual possibility of cancer death for many individuals and the cost would be the loss of land and buildings for some years or decades. At a level of ${ }^{137} \mathrm{Cs}$ ground contamination of $100 \mathrm{microcurie} / \mathrm{m}^{2}$ we get from Eq. AII.23 a lifetime dose of 155 rem without shielding, or 52 rem with the assumed shielding factor of 0.33 . (The long-term ground doses calculated in Table XLI were calculated using the prescription of Eq. AII.23 for ${ }^{137} \mathrm{Cs}$ and an analogous form for ${ }^{134} \mathrm{Cs}$.) Using the whole-body cancer death coefficients in Table XXXVIII, we see that the associated lifetime cancer death risk from remaining on the land at this contamination level would be about $0.7 \%$. For a person at 250 miles, where the integrated population dose has reached one-half the 500-mile value, the additional cancer death risk is about $0.1 \%$, and the average area which would have to be evacuated to avoid that level of risk has increased to almost $8000 \mathrm{mi}^{2}$. The authors of Draft WASH-1400 assign an average value of $\$ 2000 /$ acre (or about $\$ 4000$ /person for an average population density of $300 / \mathrm{mi}^{2}$ ) or a value of $\$ 10$ billion for $8000 \mathrm{mi}^{2}$. On an individual basis, therefore, the average person (living in the area contaminated by the plume from such a severe accident) would be faced with the choice of a $\$ 4000$ dollar loss or an extra probability of the order of 0.1 percent of dying of cancer. For society, the choice would be between of the order of 10,000 additional eventual cancer deaths (in excess of the $2,000,000$ or so which will occur in the same population from causes having nothing to do with nuclear reactors) and the long-term evacuation of $\$ 10$ billion worth of land and property $(\$ 1,000,000 /$ death avoided)-and the decision would still only involve the avoidance or acceptance of one-half the total population dose from ground contamination.

We have thus far left out a third option-that of decontamination of thousands of square miles-or at least of the more populated areas within the larger area. Unfortunately, there is not adequate information on which to base estimates of the costs or effectiveness of various decontamination procedures. [In Draft WASH-1400, Appendix VI, the statement is made that "a reasonably extensive effort, which may take a few weeks to 3 or 4 months, should be capable of a decontamination factor of between 10 and 200 or more," and reference is made to "J. W. Healy, Los Alamos Scientific Laboratory, internal publication." The document cited: "Nuclear Reactor Accident-Damage Assessment, Draft" (Healy, 1966) does not support this assertion. Since the paper is an unpublished draft, we will not discuss it further here.] Work on this subject should be pursued-not only to establish costs, but also to establish the effectiveness of various decontamination techniques. The provision of additional shielding should also be considered.

\section{Man-rem consequences}

For the reference accident which we have been discussing, Draft WASH-1400 obtains an average number of delayed deaths due to cancer of $310\left(3.1 \times 10^{6}\right.$ whole-body man-rem times 100 cancer deaths per $10^{6}$ whole-body man-rem). Other consequences considered, but not explicitly calculated, for this release are genetic defects, which would also total 310 (100 genetic defects per $10^{6}$ whole-body man-rem), and thyroid nodules. The average thyroid population dose associated with the reference accident release is not given in Draft WASH-1400, so we will use the wedge model result in Table XLI $\left(244 \times 10^{6}\right.$ thyroid rem for children under age 10) in order to estimate the dose Draft WASH-1400 would have calculated. For this comparison, the wedge model result should be reduced by a factor of approximately 3.5 because of the difference between the AEC assumptions for child thyroid dose conversion factor and breathing rates and the EPA numbers which we have used-see the discussion in connection with Table XXXVI. The resulting thyroid population dose is then $70 \times 10^{6}$ child thyroid rem which, when multiplied by the Draft WASH-1400 thyroid-nodule case probability of 333 per $10^{6}$ child thyroid rem (Appendix VI, p. 54) would give approximately 25,000 thyroid nodule cases.

In Table XLIII, we summarize the effects on the Draft WASH1400 man-rem consequences of various changes in the assumptions used there. The changes which we discuss are motivated by the preceding discussion.

From Table XLIII it will be seen that the average consequences of the reference accident assumed in Draft WASH1400 can be much larger than calculated there.

It should be apparent to the reader that there are many uncertainties in the calculation, but the additional effects which we have pointed out:

- whole-body dose from long-term ground contamination,

- lung cancer from lung beta-dose, and

- thyroid cancer deaths from thyroid doses

should be discussed in any comprehensive study of reactor accident consequences. Moreover, the effect of the uncertainties of the parameters which we have considered should also be displayed:

- child thyroid-dose conversion factor,

- cancer dose-effect coefficients,

- radionuclide release fractions from the melt, and

-genetic-consequence dose-effect coefficients.

We have not discussed here all the uncertainties in the calculation of the consequences of the Draft WASH-1400 reference accident. It would appear that more effort is needed in the exploration-of-consequences calculations before we can be certain that all important effects have been considered. The parallel and difficult task of narrowing the ranges of uncertainties of the important parameters should also be undertaken. Because the art of consequence modeling appears to be at such a rudimentary level of development, our results should not be regarded as definitive. We hope that we have contributed by having pointed out the importance of some of the effects which were not included in the calculation of Draft WASH-1400 and some of the uncertainties in the parameters used there.

\section{Other accident release categories}

The reference accident radionuclide release fractions indicated in Table XL are associated with a particular accident scenario: "failure of core cooling systems, and core melting concurrent with a loss of containment spray and heat removal systems. Failure of the containment barrier occurs through overpressure..." (Draft WASH-1400, Appendix VI, p. 10). The authors of Draft WASH-1400 conclude that this release scenario will occur in about $6 \%$ of all core melts (ibid, p. 9). Both larger and smaller releases are postulated as possible.

\section{a. Steam explosions}

The largest release of radionuclides is postulated to occur if a substantial portion of the molten core falls into a pool of water at the bottom of the reactor pressure vessel and causes a steam explosion violent enough to rupture the pressure vessel and the containment, and to disperse a portion of the core and its radioactivity as an aerosol into the atmosphere. The authors of Draft WASH-1400 assign the probability of such a steam explosion as one percent per reactor core meltdown, but this probability is repeatedly acknowledged by the authors to be a highly subjective judgement based on inadequate knowledge of the phenomena involved and uncertain by at least an order of magnitude. (Appendix VIII, pp. 27-29. A review of industrial accidents and experiments involving steam explosions is contained in Appendix B of Appendix VIII.) Obviously this is a subject in urgent need of research, especially since there may be ways to reduce the probability of steam explosions. 
TABLE XLIII. Effect of changed assumptions on Draft WASH-1400 reference accident average man-rem consequences.

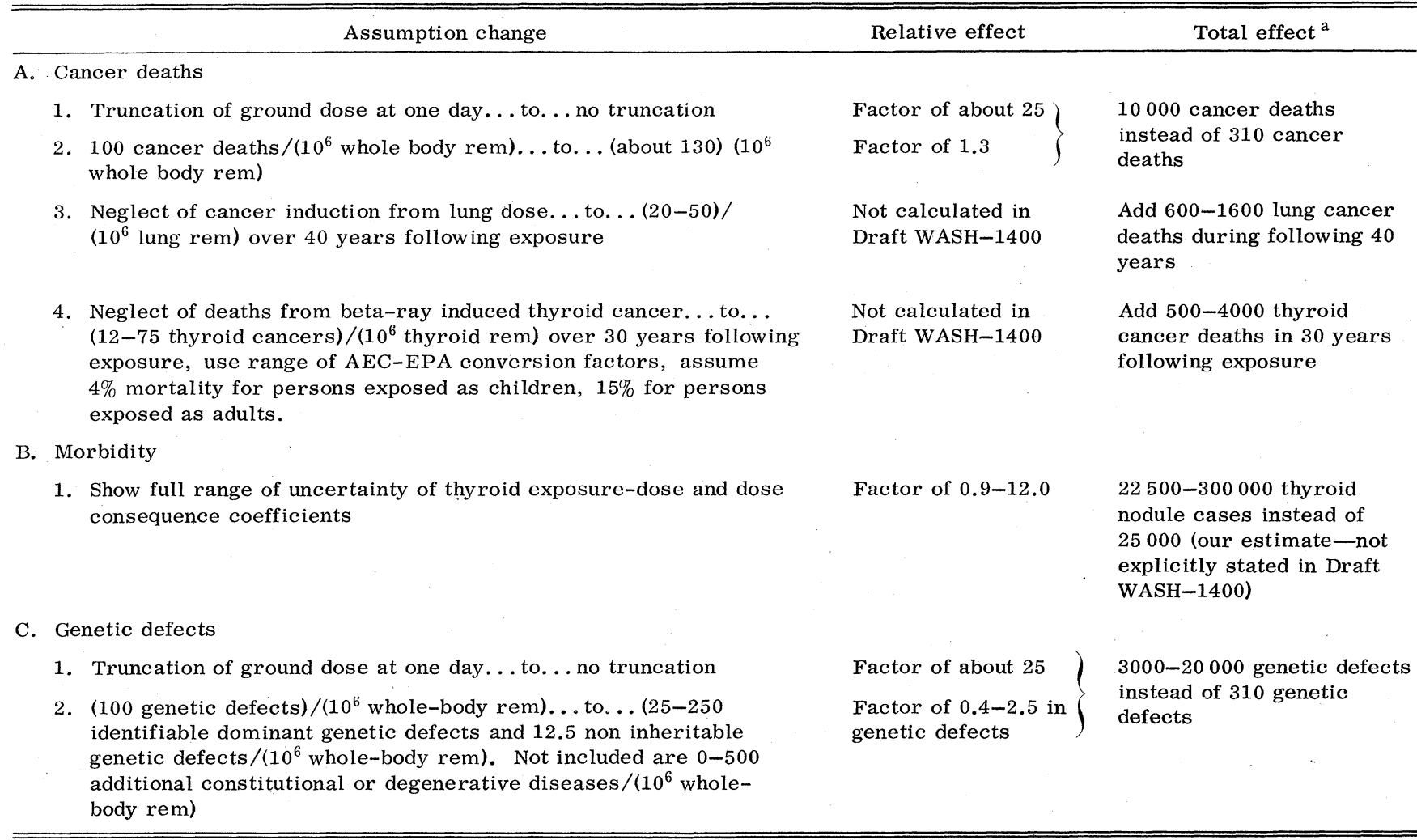

${ }^{a}$ It is essential to note that these incremental cancer deaths and morbidity would occur over natural lifetimes of a very large exposed population. These calculations are based on a population density of $300 / \mathrm{mi}^{2}$ which results in an exposure of a population of about 10 million. A population density of $165 / \mathrm{mi}^{2}$ would multiply all total consequence estimates by 0.55 (see footnote, page $\mathrm{V}-59$ ). Independent of assumed population density, there would be an additional risk of cancer to the average exposed individual of one chance in a thousand, with the risk distributed over a substantial fraction of his natural lifetime.

\section{b. Melt-through}

The authors of Draft WASH-1400 judge that for a PWR meltdown, the most probable release is much less than occurs in the reference accident, because they expect that $85 \%$ of the time the containment would not fail until the core melted through the base of the containment. (See Appendix VIII.) Radioactive gases and aerosols would, therefore, not reach the atmosphere until they had percolated through the soil to the surface beyond the containment building. A reduction by a factor 100 to 1000 , relative to the reference accident, in the atmospheric release of radionuclides is assumed to result for all fission products (other than the noble gases) as a result of the filtering action of the soil. The consequences are, therefore, expected to be relatively minor.

That the soil would do a substantial amount of filtering in such a melt-through seems plausible, but we are concerned about the possibility that blowouts or channeling might partially short-circuit this filtration system. The driving forces which could cause such a blowout are:

(1) the gas pressure within the containment which, it is assumed, can rise to $100 \mathrm{psi}$ in a PWR before the containment fails above ground (Draft WASH-1400, Appendix VIII) and

(2) pressure pulses from steam explosions as the molten core contacts water in the soil. (An explosion occurred when molten steel fell onto damp ground at Armco Steel in 1967. See Draft WASH-1400, Appendix VIII, p. B-2.) These pressures would have to work against the hydrostatic pressure of the soil-about 33 psi for an average soil density of $118 \mathrm{1b} / \mathrm{ft}^{3}$ and a depth of 40 feet (Draft WASH-1400, Appendix VII, Appendix K). The authors of Draft WASH-1400 argue that channeling will occur because "the interfaces between several layers of different materials should act as crack arrestors..." (Appendix VII, p. 54). However, it is our understanding that many plants are based on alluvial material without such a layered structure. Also, channeling might occur along the interface between the cofferdam surrounding the containment building and the soil. Before credit for ground filtration factors as large as 100 to 1000 can be taken, these questions will have to be addressed and reliably answered. It seems reasonable that some credit for such filtration should be taken, however, and that this protection could be augmented by attention to it during reactor design and construction.

\section{Property damages}

We have thus far discussed the health consequences of land contamination. However, contamination with radionuclides can make land unsuitable for agriculture even if it is considered suitable for human occupation. In particular, Table XXXIX indicates that the authors of Draft WASH-1400 have suggested agriculture contamination thresholds, for ${ }^{90} \mathrm{Sr}$ and ${ }^{137} \mathrm{Cs}$ respectively, 10 times and 5 times lower than for human occupation. For the reference accident release the wedge model gives the corresponding average contamination threshold radius as approximately 160 miles. The area within this sector is about $3200 \mathrm{mi}^{2}$. Draft WASH-1400 does not give the corresponding cost numbers. It is stated there, however, that "The area over which one year's crops would need to be monitored 
for possible confiscation is approximately 10 times larger than the evacuation area" (Appendix VI, p. 77). We previously calculated the evacuation area for the PWR2 accident to be approximately $200 \mathrm{mi}^{2}$. We perform another check as follows: In Appendix VI on p。 70 (Draft WASH-1400), two-thirds of the cost of the "average accident" is attributed to land contamination. If this applies to the reference accident whose total cost is given as $\$ 1.8 \times 10^{9}$, then land contamination accounts for $\$ 1.2 \times 10^{9}$ of damage. The authors of Draft WASH-1400 assume a half-life for this land contamination of one year, indicating that most of this land will be out of use for only one year (Appendix VI, p. 66). For each year out of use up to 10 years they assume a corresponding loss of about $\$ 1.3 \times 10^{5} / \mathrm{mi}^{2}$. The cost $\$ 1.2 \times 10^{9}$, therefore, corresponds to the loss for one year of approximately $10,000 \mathrm{mi}^{2}$.

However, it should be recalled here that the natural decontamination half-lives for crop-land contamination by ${ }^{90} \mathrm{Sr}$ and ${ }^{137} \mathrm{Cs}$, for which we have found documentation (See Sec. V above), are of the order of 5-6 years and a few years, respectively (and not just 1 year). For the ${ }^{137} \mathrm{Cs}$ ground dose the half-life is of the order of 10 years. The reference cited in Draft WASH-1400 for the 1 year value used there does not contain it. The damage estimates cited in Draft WASH-1400 should, therefore, probably be increased by corresponding factors. The thresholds are so arbitrary and the possibilities for land decontamination or reassignment so unevaluated, however, that we cannot here provide a definitive estimate. It is apparent that more careful estimates must be made, and a less dichotomous choice made between "agricultural use" and "non-agricultural" use. For instance, one could grow timber on some contaminated land, which would reduce its value, but not to zero.

\section{Ground water contamination}

Since reactors are typically located near the edges of rivers, lakes, and estuaries and these bodies of water are often important sources of drinking water or food, the potential for water contamination from a reactor accident becomes an important issue.

This problem has been discussed in Draft WASH-1400 Appendix VII, pp. 55-63. It is assumed there that the molten core cools to form a cylindrical glasslike mass approximately 70 feet in diameter and 60 feet high in the ground beneath the reactor containment. After the outside of the mass has cooled below the boiling temperature of water, leaching is assumed to begin.

The integrated diffusion out of a sphere of radius $R$ of an element uniformly distributed in the sphere with a diffusion coefficient $D$ is given for small times by (Crank, 1956, p. 87)

Integrated Fraction Release $\approx 6\left(\frac{D t}{\pi R^{2}}\right)^{1 / 2}, t \ll \frac{R^{2}}{D}$.

The authors of Draft WASH-1400 assume that the diffusion constant for ${ }^{90} \mathrm{Sr}$ out of the glass corresponds to that measured for basalt. The diffusion constants measured for basalt lie in a range of approximately $10^{-16}$ to $10^{-14} \mathrm{~cm}^{2} / \mathrm{sec}$. depending upon temperature (factor of 4 to 30 between $30^{\circ}$ and $90^{\circ} \mathrm{C}$ ) and author (up to a factor 3) (Saidl and Rakova, 1966). The leaching of ${ }^{90} \mathrm{Sr}$ for the peak year (following the time at which the cooling is sufficient for leaching to become significant) is calculated in Draft WASH-1400 to be of the order of one part in $10^{5}$. Using this value and a value of $D=10^{-15}$ and $t=3.15 \times 10^{7}$ seconds (1 year) in Eq. VI-1, we may obtain a rough estimate of the equivalent radius used in Draft WASH-1400. We find $R=60 \mathrm{~cm}$. This equivalent radius indicates to us that the authors of Draft WASH-1400 must have assumed that the cracks in the melt caused by thermal stresses, inhomogeneities, steam explosions, etc. during cooling had an average spacing of the order of one meter. It would be appropriate to have documen- tation of this assumption-it seems to us to be somewhat large. We have no alternate value to offer, but note that if $R$ were reduced to $1 \mathrm{~cm}$ the leach rate would increase by two orders of magnitude. It is important that a more refined analysis be undertaken including a review of the appropriateness of using the diffusion constant of basalt.

A serious problem with this analysis is that it neglects the water contamination due to all of the radionuclides which were assumed in another part of Draft WASH-1400 (see above) to be released in gaseous and aerosol form from the melt. In some cases, these gases and aerosols might well follow the core through the base mat and be trapped in the soil around the base of the containment. These aerosols are assumed to contain about 6 percent of the core inventory of strontiums. It seems likely that this fraction of these radionuclides will be much more mobile than that trapped in the melt.

The movement of ${ }^{90} \mathrm{Sr}$ in ground water is likely to be retarded by ion-exchange phenomena but its movement may be significant. In a study of the ground water under the Idaho Chemical Processing Plant at the Idaho National Engineering Laboratory in Idaho it was found that ${ }^{90} \mathrm{Sr}$ injected into an aquifer through a deep well had migrated approximately a kilometer in 8 years (Robertson and Barraclough, 1973).

The average distance of a reactor from a nearby large body of water assumed in Draft WASH-1400 (Appendix VII, p. 56) is 1500 feet. The above observations indicate that water-supply contamination might be a very serious hazard in case of a reactor core meltdown. Obviously a more sophisticated treatment of this problem is called for. The feasibility of measures to mitigate ground water contamination, such as pumping the water out and treating it or treating the soil to reduce its permeability, should also be explored.

\section{F. Mitigating the consequences}

The preceding discussion focuses on the consequences of a reactor accident without considering how these consequences might be mitigated. We are confident that methods could be found to reduce some of these consequences substantially. We discuss four possibilities here:

\section{a. Jodine blocking}

One method to reduce the uptake of radioiodine by the thyroid already seems to have been rather well documented in a Civil Defense study (Cole, 1972). It appears from this review that the uptake of radioiodine by adult thyroids could be reduced by an order of magnitude if a "blocking". or diluting dose of $100 \mathrm{mg}$ or so of stable iodine were taken at the time of, or within a few hours before, exposure. For an exposure stretching over days or weeks, further blocking doses could be taken without a high incidence of serious side effects. Cole estimates that enough 100-mg KI pills to provide a week's worth of blocking dosage for the entire population of the U. S. (six 100-mg tablets per capita) would cost about $\$ 1$ million. This would obviously be cheap insurance if it would make possible a tenfold reduction of the tens of thousands of thyroid cancers and nodules which might result from a major reference accident type of release of radionuclides. How much reduction could be achieved in practice would depend upon an assessment of the potential thyroid radiation dose level at which the danger of side effects exceeds the danger of the thyroid dose. In this connection we note that the reference accident wedge-model child thyroid dose 200 miles downwind is about $500 \mathrm{rem}$ and brings along with it (see Table XXXVIII) a probability of thyroid nodules of 10 to $60 \%$. (All the Marshallese and about one third of the Rochester nodule cases were operated upon.) It seems likely that iodine blocking would be found beneficial out to considerable distances. We believe that a national policy of stockpiling thyroid blocking chemicals for possible emer- 
gency distribution should be established. More research will probably be required to establish the appropriate dose and potential side effects for children.

\section{b. Diversion of contaminated food supplies}

Another mitigating effect has already been assumed throughout our discussion above. This is that fallout-contaminated food and milk would be diverted until its contamination had dropped to low levels. Such diversion would not totally eliminate the population dose from this source but using the contamination thresholds set in Table XXXIX, we believe that the long-term integrated dose via this route would be considerably less than that due to direct gamma radiation from the contaminated ground. In the short term, however, and in the absence of provisions for diversion, the ${ }^{131}$ I dose to the child's thyroid via milk produced in the same area would be of the order of 50 times higher than directly through inhalation (EPA, 1973, p. 127).

\section{c. Decontamination and evacuation}

The major long-term contribution to population whole-body dose would come from land contamination. We are convinced that it is important to develop decontamination procedures which could decrease this whole-body exposure severalfold and to establish improved guidelines for evacuation from contaminated areas. The level suggested in the Draft WASH-1400 study, 10 rem in the first year, is 20 times higher than the maximum dose which it is currently assumed permissible for an individual member of the public to receive from peaceful uses of atomic energy. This may be realistic in view of the fact that the area which would have to be evacuated would go roughly inversely as the square of the assumed contamination threshold, but it is important that the question of evacuation thresholds be addressed seriously and openly.

\section{d. Air filtration}

Immediate deaths would be expected to result primarily from internal radiation doses received by persons within a few tens of miles of an accident, after inhalation of the radioactive aerosol. The authors of Draft WASH-1400 assume that these consequences can be substantially reduced by evacuation. However, rapid evacuation might not be possible in built-up areas, and the advantages of the availability of improved air filtration in large buildings and breathing masks in the neighborhood of nuclear power plants might be considered. In view of the nonlinear dependence of immedia te deaths on dose, a factor of 10 reduction in the inhaled dose could reduce the number of shortterm deaths by a far larger factor. Thus the possibility of reducing acute effects to a far lower level by use of breathing masks by the localized population exposed to the acute hazard, should be investigated.

\section{G. Improved containment systems}

The potential importance of improved containment concepts will be obvious from the above discussion of the potential consequences of an uncontained meltdown accident. Below we briefly discuss some of the possibilities for such improvement which we believe would be worth exploring.

\section{Controlled failure}

Draft WASH-1400 assumed that the containment would ultimately fail if the reactor core melts down. If failure did not occur through overpressure, it would occur when the core melted through the bottom of the containment shell, in a period of the order of a day (Draft WASH-1400, Appendix VIII).
The manner in which the containment failed, and the effectiveness with which the fission products would be scrubbed out of the containment before failure, are very important, however. Thus if the containment spray system in a PWR worked as designed, it would be expected to reduce the release of fission products by an order of magnitude in a core-melt accidenteven if the containment were inadequately isolated. (See the discussion in Draft WASH-1400, Appendix V-especially p. 147.) (A concern which we have been unable to resolve, however, relates to the ultimate fate of fission products which have been scrubbed out into water. Is the water a final resting place, or is there a possibility that the water will be evaporated and the fission products become airborne once again?) Similarly, in PWR accidents where the containment might fail due to melt-through by the core, rather than by overpressure, the total fission product release is assumed to be reduced greatly as a result of the filtering action of the soil (Draft WASH-1400, Appendix V).

These possible effects suggest that emphasis be placed on containment designs which ensure that, in case of containment failure, fission products would be scrubbed and/or filtered before their release. Thus, for example, a BWR containment might be designed to fail in a controlled way via pressure release valves which vented from the pressure-suppression pool or through large stabilized-bed filters. The remaining gases could then be filtered through the standby gas treatment system.

\section{Underground siting}

The advantages and disadvantages of underground siting have been vigorously debated over the years, but we believe that they have still not been studied in adequate depth. Studies which have been made, however, indicate that the extra costs might not be prohibitive and that the possibility merits serious consideration. We list a few of the reasons here:

(i) A Lawrence Livermore Laboratory study asserts that it is not necessary to find a large volume of solid rock out of which to mine the reactor containment. It suggests instead that it might be economic to dig an open pit 300 feet deep, build the containment, and then refill around and over (Blake et $a l_{.}, 1973$ ).

(ii) A United Engineers study suggests that the earthquake stresses on an underground structure might be much less severe than on a surface structure. This is because a surface structure has some of the properties of an inverted pendulum and can amplify the motions of the subsurface rock by more than an order of magnitude. An underground structure, on the other hand, is directly coupled into the motions of the subsurface rock (Crawley and McCreath, 1972). For a discussion of earthquake motion amplification in surface structures see, e.g., T. F. Lomanik (1970).

(iii) An obvious property of an underground containment is that, because of the load of the overburden, burying a containment a few hundred feet deep can raise the threshold for its failure due to internal overpressure by a factor of two or so. (See above two references and also Watson et al., 1972.) This advantage will be compensated in part, however, by the fact that the containment might be expected to have a volume smaller by a factor of 2 or so than that of a comparable surface plant. Consequently, it would take only twice as much energy (and twice as much water) to overpressure an underground containment which might have a failure pressure of 400 psi, than the surface containment which has a failure pressure of 100 psi. Sufficient energy could be deposited in the atmosphere of the underground cavity to generate 400 psi overpressure within a few hours. (See, e.g., Beck and Watson, 1973.) Undergrounding a containment would therefore not eliminate the need for providing a heat sink; it would only stretch out the time period before it is needed.

(iv) If a heat sink were provided, the melt-through of the 
base of the containment would be less serious than the corresponding failure of a surface containment because of the greater distance over which fission-product gases would have to seep before reaching the surface. In this connection it should be noted that the rate at which gas under pressure penetrates soil can vary by orders of magnitude. For a pressure gradient of one psi per foot (2400 dynes/c, ${ }^{3}$ ) the rate of penetration by air or similar gas will be of order $v=2.5 \times 10^{-5} k / u E \mathrm{~cm} / \mathrm{sec}$. (See Draft WASH-1400, Appendix VII, p. k-10.) Here $E$ is the porosity of the soil $(E \approx 0.5), \mu$ is the viscosity of air $\left(\approx 2 \times 10^{-4} \mathrm{gm} / \mathrm{cm}-\mathrm{sec}\right)$ and $k$ is the permeability of soil measured in "darcies" (one darcy $=0.987 \times 10^{-8} \mathrm{~cm}^{2}$ ). Substituting in the equation above for $E$ and $\mu$ we get

$$
v=0.25 k \mathrm{~cm} / \mathrm{sec}
$$

The value of $k$ can vary from of order 10 for sand down to of order $10^{-2}$ for clay. [See, e.g., DeWiest, (1969) pp. 80, 81.] For $k=10^{-2}$ and a depth of 100 meters it could take weeks for the first redioactive seepage to reach the surface, thus allowing time for decay, for chemical treatment of the surface, etc.

(v) A surface reservoir of water will have a head of the order of 100 psi relative to a cavity 200 feet underground. It might be possible to take advantage of this fact in the design of a passive heat exchanger for the containment (e.g., an arrangement by which the water flows down under the influence of gravity and flows back to the surface as steam). There need be no fear that the hydrostatic pressure will rupture the containment, since the lithostatic pressure will always be higher.

(vi) An underground containment would be protected against many natural and man-made threats: tornadoes, aircraft, missiles, and even, to some extent, nuclear weapons.

[For a general review of underground and off-shore siting proposals see Yadigaroglu and Andersen (1974).]

\section{Core catcher}

Since containments as currently designed would all fail by melt-through, one might well ask if it is possible to design a "core catcher" which would hold the molten core and prevent a melt-through. Studies of the possibilities for doing this have been done (AEC/ECCS, 1967; Morrison et al., 1971; Jansen and Stepnewski, 1973). In view of the relatively large probability (of the order of $10^{-4}$ per reactor year) of a reactor core meltdown given in Draft WASH-1400, we suggest that more advanced studies on a few of the more promising concepts be pursued.

\section{H. Siting policies}

If it is impossible to design a perfect containment, an alternative is that of siting the reactor far away from large human populations or valuable properties. How far away would one have to go? Our discussion indicates that it would be very difficult to locate a reactor so remotely that the hazard measured in population man-rem would be reduced by a large factor. One might consider another philosophy, however, and choose a site remote enough so that the hazard to individuals becomes small. The most stringent constraints on siting would be imposed by thyroid and ground contamination effects. This again emphasizes the importance of iodine blocking and decontamination countermeasures.

Siting at distances of the order of 500 miles from load centers is not impractical. Power is already routinely transmitted for such distances (from the Columbia River and Four Corners to California, for example). The option of remote siting seems worthy of further exploration.

If remote siting were adopted, it would seem reasonable to explore the possibility of siting a considerable number of power plants at one location. The advantages and disadvantages of such "nuclear energy centers" have been explored recently in a preliminary AEC study (AEC, WASH-1288, 1974) and are the subject of other current studies (see, e.g., "Assessment of Energy Parks vs. Dispersed Electric Power Generating Facilities," NSF-funded study at the General Electric Company, Center for Energy Systems, Washington, D. C.; Nuclear Regulatory Commission study). 
APS study group on light-water reactor safety

Rev. Mod. Phys., Vol. 47, Suppl. No. 1, Summer 1975 


\section{APPENDIX III. DESCRIPTION OF THE ECCS ACCEPTANCE CRITERIA}

The following reproduced directly from the Atomic Energy Commission's document REGULATORY ADJUDICATION ISSUANCES, RAI-73-12, January, 1974, pages 1129-1138.

It contains a description of the ECCS ACCEPTANCE CRITERIA.

On November 30, 1971, the Atomic Energy Commission published in the Federal Register (36 F.R. 22774) a notice scheduling a legislative-type public rule making hearing on January 27,1972 , before a hearing board consisting of Nathaniel H. Goodrich, Esq., Chairman, Dr. Lawrence R. Quarles, and Dr. John H. Buck, concerning its interim statement of policy establishing acceptance criteria for emergency core cooling systems for light water-cooled nuclear power reactors, published June 29, 1971 (36F.R. 12247). Amendments to the interim criteria were published in the Federal Register on December 18, 1971 (36 F.R. 24082) in a notice that stated that the amendments would also be considered at the rule making hearing.

Participation in the rule making hearing was extensive. The primary participants included the Commission Regulatory Staff, four reactor manufacturers, a consolidated group of electric utility companies, and the Consolidated National Intervenors (CNI), a group of about 60 organizations and individuals. In addition, three states, the Lloyd Harbor Study Group, and several individuals participated to a lesser degree. The hearings lasted a total of 125 days and generated a record of more than 22,000 pages of transcript and thousands of pages of written direct testimony and exhibits. Oral argument from the seven principal participants was heard by the Commission on October 9, 1973.

In implementation of the National Environmental Policy Act of 1969, (P.L. 91-190), a Draft Environmental Statement concerning the proposed rule making was forwarded to the Council on Environmental Quality on December 6, 1972, and circulated for comment to participants in the hearing and interested Federal Agencies on December 7, 1972. Notice of public availability of the Statement and an invitation for comment was also published in the Federal Register at that time. Comments on the Draft Statement were received and a Final Environmental Statement was published on May 9, 1973.

The Commission noted in the interim Policy Statement:

Protection against a highly unlikely loss-of-coolant accident has long been an essential part of the defense-in-depth concept used by the nuclear power industry and the AEC to assure the safety of nuclear power plants. In this concept, the primary assurance of safety is accident prevention by correctly designing, constructing, and operating the reactor. Extensive and systematic quality assurance practices are required and applied at every step to achieve this primary assurance of safety. Nevertheless, deviations from expected behavior are postulated to occur, and protective systems are installed to take corrective action as required in such events. Notwithstanding all this, the occurrence of serious accidents is postulated, in spite of the fact that they are highly unlikely, and engineered safety features are installed to mitigate the consequences of these unlikely events. The loss-of-coolant accident is such a postulated improbable accident; the emergency core cooling system is one of the engineered safety features installed to mitigate its consequences.

The Commission has adopted new regulations, set forth below, dealing with the effectiveness of ECCS. In a 140 page opinion issued on December 28, 1973, the Commission discussed the changes from the interim acceptance criteria and the technical reason for them. Copies of this opinion are available for inspection and copying at the Commission's Public Document Room, 1717 H. Street, N. W., Washington, D. C.

The principal changes from the Interim Policy Statement are as follows. The old criterion number one, specifying that the temperature of the Zircaloy cladding should not exceed $2300^{\circ} \mathrm{F}$, is replaced by two criteria, lowering the allowed peak Zircaloy temperature to $2200^{\circ} \mathrm{F}$ and providing a limit on the maximum allowed local oxidation. The other three criteria of the IAC are retained, with some modification of the wording. These three criteria limit the hydrogen generation from metal-water reactions, require maintenance of a coolable core geometry, and provide for long-term cooling of the quenched core.

The most important effect of the changes in the required features of the evaluation models is that swelling and bursting of the cladding must now be taken into consideration when they are calculated to occur, and that the maximum temperature and oxidation criteria must be applied to the region of clad swelling or bursting when the maximum temperature and oxidation are calculated to occur there. Another important change is the requirement that, in the steady state operation just before the postulated accident, the thermal conductance of the gap between the fuel pellets and the cladding should be calculated taking into consideration any increase in gap dimensions resulting from such phenomena as fuel densification, and should also consider the effects of the presence of fission gases. When these effects are taken into consideration a higher stored energy may be calculated. Other changes in the evaluation models are mostly in the direction of replacing previous broad conservative assumptions with more detailed calculations where new experimental information is available or where better calculational methods have been developed.

The wording of the definition of a loss-of-coolant accident has been modified to conform to its long-accepted usage, limiting it to breaks in pipes. The new regulations also require a more complete documentation of the evaluation models that are used. 
The Commission believes that the implementation of the new regulations will ensure an adequate margin of performance of the ECCS should a design basis LOCA ever occur. This margin is provided by conservative features of the evaluation models and by the criteria themselves. Some of the major points that contribute to the conservative nature of the evaluations and the criteria are as follows:

(1) Stored Heat. The assumption of $102 \%$ of maximum power, highest allowed peaking factor, and highest estimated thermal resistance between the $\mathrm{UO}_{2}$ and the cladding provides a calculated stored heat that is possible but unlikely to occur at the time of a hypothetical accident. While not necessarily a margin over the extreme condition, it represents at least an assumption that an accident happens at a tume which is not typical.

(2) Blow-down. The calculation of the heat transfer during blowdown is made in a very conservative manner. There is evidence that more of the stored heat would be removed than calculated, although there is not yet an accepted way of calculating the heat transfer more accurately. It is probable that this represents a conservatism of several hundred degrees $\mathrm{F}$ in stored energy after blowdown, most of which can reasonably be expected to carry over to a reduction in the calculated peak temperature of the Zircaloy cladding.

(3) Rate of Heat Generation. It is assumed that the heat generation rate from the decay of fission products is $20 \%$ greater than the proposed ANS standard. This represents an upper limit to the degree of uncertainty. The assumption that the fission product level is that resulting from operation at $102 \%$ of rated power for an infinite time represents an improbable situation, with a conservatism that is probably in the range of 5 to $15 \%$. The use of the Baker-Just equation for calculating the heat generation from the steam oxidation of zircaloy should also provide some conservatism, but the factor is uncertain.

(4) The Peak Temperature Criterion. The limitation of the peak calculated temperature of the cladding to $2200^{\circ} \mathrm{F}$ and the stipulation that this criterion be applied to the hottest region of the hottest fuel rod provide a substantial degree of conservatism. They ensure that the core would suffer very little damage in the accident.

Pursuant to the Atomic Energy Act of 1954, as amended, and Sections 552 and 553 of Title 5 of the United States Code, the following amendments to Title 10, Chapter I, Code of Federal Regulations, Part 50, are published as a document subject to codification to be effective on [30 days after publication in the Federal Register].

1. A new sentence is added to Section 50.34(a)(4) of 10 CFR Part 50 to read as follows:

$\S 50.34$ Contents of applications: technical information

(a) $* *$

(4) *** Analysis and evaluation of ECCS cooling performance following postulated loss-of-coolant accidents shall be performed in accordance with the requirements of $\S 50.46$ for facilities for which construction permits may be issued after December $28,1974$.

2. A new sentence is added to Section 50.34(b)(4) 10 CFR Part 50 to read as follows:

$\$ 50.34$ Contents of applications; technical information.

(a) $* * *$

(b) $* * *$

(4) *** Analysis and evaluation of ECCS cooling performance following postulated loss-of-coolant accidents shall be performed in accordance with the requirements of $\$ 50.46$ for facilities for which a license to operate may be issued after December 28, 1974

3. A new $\S 50.46$ is added to 10 CFR Part 50 to read as follows:

$\$ 50.46$ Acceptance Criteria for Emergency Core Cooling Systems for Light Water Nuclear Power Reactors.

(a)(1) Except as provided in subparagraphs (2) and (3) of this paragraph, each boiling and pressurized light-water nuclear power reactor fueled with uranium oxide pellets within cylindrical zircaloy cladding shall be provided with an emergency core cooling system (ECCS) which shall be designed such that its calculated cooling performance following postulated loss-of-coolant accidents conforms to the criteria set forth in paragraph (b). ECCS cooling performance shall be calculated in accordance with an acceptable evaluation model, and shall be calculated for a number of postulated loss-of-coolant accidents of different sizes, locations, and other properties sufficient to provide assurance that the entire spectrum of postulated loss-of-coolant accidents is covered. Appendix K, ECCS Evaluation Models, sets forth certain required and acceptable features of evaluation models. Conformance with the criteria set forth in paragraph (b), with ECCS cooling performance calculated in accordance with an acceptable evaluation model, may require that restrictions be imposed on reactor operation.

(2) With respect to reactors for which operating licenses have previously been issued and for which operating licenses may issue on or before December 28, 1974:

(i) The time within which actions required or permitted under this subparagraph (2) must occur shall begin to run on [ 30 days after publication of the rule in the Federal Register]

(ii) Within six months following the date specified in subparagraph (i) of this subparagraph (2), an evaluation in accordance with subparagraph (1) of this paragraph (a) shall be submitted to the Director of Regulation. The evaluation shall be accompanied by such proposed changes in technical specifications or license amendments as may be necessary to bring reactor operation in conformity with subparagraph (1) of this paragraph.

(iii) Any licensee may request an extension of the six-month period referred to in subparagraph (ii) of this subparagraph (2) for good cause. Any such request shall be submitted not less than 45 days prior to expiration of the six-month period, and shall be accompanied by affidavits showing precisely why the evaluation is not complete and the minimum time believed necessary to complete it. The Director of 
Regulation shall cause notice of such a request to be published promptly in the Federal Register; such notice shall provide for the submission of comments by interested persons within a time period to be established by the Director of Regulation. If, upon reviewing the foregoing submissions, the Director of Regulation concludes that good cause has been shown for an extension, he may extend the six-month period for the shortest additional time which in this judgment will be necessary to enable the licensee to furnish the submissions required by subparagraph (ii) of this subparagraph (2). Requests for extensions of the six-month period, submitted under this subparagraph, shall be ruled upon by the Director of Regulation prior to expiration of that period.

(iv) Upon submission of the evaluation required by subparagraph (ii) of this subparagraph (2) (or under subparagraph (iii), if the six-month period is extended) the facility shall continue or commence operation only within the limits of both the proposed technical specifications or license amendments submitted in accordance with this subparagraph (2) and all technical specifications or license conditions previously imposed by the Commission, including the requirements of the Interim Policy Statement (June 29, 1971, 36 F.R. 12248), as amended (December 18, 1971, 36 F.R. 24082).

(v) Further restrictions on reactor operation will be imposed by the Director of Regulation if he finds that the evaluations submitted under subparagraphs (ii) and (iii) of this subparagraph (2) are not consistent with subparagraph (1) of this paragraph (a) and as a result such restrictions are required to protect the public health and safety.

(vi) Exemptions from the operating requirements of subparagraph (iv) of this subparagraph (2) may be granted by the Commission for good cause. Requests for such exemption shall be submitted not less than 45 days prior to the date upon which the plant would otherwise be required to operate in accordance with the procedures of said subparagraph (iv). Any such request shall be filed with the Secretary of the Commission, who shall cause notice of its receipt to be published promptly in the Federal Register; such notice shall provide for the submission of comments by interested persons within 14 days following Federal Register publication. The Director of Regulation shall submit his views as to any requested exemption within five days following expiration of the comment period.

(vii) Any request for an exemption submitted under subparagraph (vi) of this subparagraph (2) must show, with appropriate affidavits and technical submissions, that it would be in the public interest to allow the licensee a specified additional period of time within which to alter the operation of the facility in the manner required by subparagraph (iv) of this subparagraph (2). The request shall also include a discussion of the alternatives available for establishing compliance with the rule.

(3) Construction permits may be issued after December 28, 1973 but before December 28, 1974 subject to any applicable conditions or restrictions imposed pursuant to other regulations in this chapter and the Interim Acceptance Criteria for Emergency Core Cooling Systems published on June 29, 1971 (36 F.R. 12248) as amended (December 18, 1971, 36 F.R. 24082): Provided, however, that no operating license shall be issued for facilities constructed in accordance with construction permits issued pursuant to this subparagraph, unless the Commission determines, among other things, that the proposed facility meets the requirements of subparagraph (1) of this paragraph.

(b)(1) Peak Cladding Temperature. The calculated maximum fuel element cladding temperature shall not exceed $2200^{\circ} \mathrm{F}$.

(2) Maximum Cladding Oxidation. The calculated total oxidation of the cladding shall nowhere exceed 0.17 times the total cladding thickness before oxidation. As used in this subparagraph total oxidation means the total thickness of cladding metal that would be locally converted to oxide if all the oxygen absorbed by and reacted with the cladding locally were converted to stoichiometric zirconium dioxide. If cladding rupture is calculated to occur, the inside surfaces of the cladding shall be included in the oxidation, beginning at the calculated time of rupture. Cladding thickness before oxidation means the radial distance from inside to outside the cladding, after any calculated rupture or swelling has occurred but before significant oxidation. Where the calculated conditions of transient pressure and temperature lead to a prediction of cladding swelling, with or without cladding rupture, the unoxidized cladding thickness shall be defined as the cladding cross-sectional area, taken at a horizontal plane at the elevation of the rupture, if it occurs, or at the elevation of the highest cladding temperature if no rupture is calculated to occur, divided by the average circumference at that elevation. For ruptured cladding the circumference does not include the rupture opening.

(3) Maximum Hydrogen Generation. The calculated total amount of hydrogen generated from the chemical reaction of the cladding with water or steam shall not exceed 0.01 times the hypothetical amount that would be generated if all of the metal in the cladding cylinders surrounding the fuel, excluding the cladding surrounding the plenum volume, were to react.

(4) Coolable Geometry. Calculated changes in core geometry shall be such that the core remains amenable to cooling.

(5) Long-Term Cooling. After any calculated successful initial operation of the ECCS, the calculated core temperature shall be maintained at an acceptably low value and decay heat shall be removed for the extended period of time required by the long-lived radioactivity remaining in the core.

(c) As used in this section:

(1) Loss-of-coolant accidents (LOCA's) are hypothetical accidents that would result from the loss of reactor coolant, at a rate in excess of the capability of the reactor coolant makeup system, from breaks in pipes in the reactor coolant pressure boundary up to and including a break equivalent in size to the double-ended rupture of the largest pipe in the reactor coolant system.

(2) An evaluation model is the calculational framework for evaluating the behavior of the reactor 
system during a postulated loss-of-coolant accident (LOCA). It includes one or more computer programs and all other information necessary for application of the calculational framework to a specific LOCA, such as mathematical models used, assumptions included in the programs, procedure for treating the program input and output information, specification of those portions of analysis not included in computer programs, values of parameters, and all other information necessary to specify the calculational procedure.

(d) The requirements of this section are in addition to any other requirements applicable to ECCS set forth in this Part. The criteria set forth in paragraph (b), with cooling performance calculated in accordance with an acceptable evaluation model, are in implementation of the general requirements with respect to ECCS cooling performance design set forth in this Part, including in particular Criterion 35 of Appendix A.

4. A new Appendix K is added to 10 CFR Part 50 to read as follows: Appendix K-ECCS Evaluation Models.

I. Required and Acceptable Features of Evaluation Models.

II. Required Documentation.

\section{REQUIRED AND ACCEPTABLE FEATURES OF THE EVALUATION MODELS}

\section{A. SOURCES OF HEAT DURING THE LOCA}

For the heat sources listed in Paragraphs 1 to 4 below it shall be assumed that the reactor has been operating continuously at a power level at least 1.02 times the licensed power level (to allow for such uncertainties as instrumentation error), with the maximum peaking factor allowed by the technical specifications. A range of power distribution shapes and peaking factors representing power distributions that may occur over the core lifetime shall be studied and the one selected should be that which results in the most severe calculated consequences, for the spectrum of postulated breaks and single failures analyzed.

1. The Initial Stored Energy in the Fuel. The steady-state temperature distribution and stored energy in the fuel before the hypothetical accident shall be calculated for the burn-up that yields the highest calculated cladding temperature (or, optionally, the highest calculated stored energy). To accomplish this, the thermal conductivity of the $\mathrm{UO}_{2}$ shall be evaluated as a function of burn-up and temperature, taking into consideration differences in initial density, and the thermal conductance of the gap between the $\mathrm{UO}_{2}$ and the cladding shall be evaluated as a function of the burn-up, taking into consideration fuel densification and expansion, the composition and pressure of the gases within the fuel rod, the initial cold gap dimension with its tolerances, and cladding creep.

2. Fission Heat. Fission heat shall be calculated using reactivity and reactor kinetics. Shutdown reactivities resulting from temperatures and voids shall be given their minimum plausible values, including allowance for uncertainties, for the range of power distribution shapes and peaking factors indicated to $t$ studied above. Rod trip and insertion may be assumed if they are calculated to occur.

3. Decay of Actinides. The heat from the radioactive decay of actinides, including neptunium an plutonium generated during operation, as well as isotopes of uranium, shall be calculated in accordanc with fuel cycle calculations and known radioactive properties. The actinide decay heat chosen shall be tha appropriate for the time in the fuel cycle that yields the highest calculated fuel temperature during th LOCA.

4. Fission Product Decay. The heat generation rates from radioactive decay of fission products shall b assumed to be equal to 1.2 times the values for infinite operating time in the ANS Standard (Propose American Nuclear Society Standard-"Decay Energy Release Rates Following Shutdown of Uranium Fueled Thermal Reactors", Approved by Subcommittee ANS-5, ANS Standards Committee, Octobe 1971). The fraction of the locally generated gamma energy that is deposited in the fuel (including th cladding) may be different from 1.0; the value used shall be justified by a suitable calculation.

5. Metal-Water Reaction Rate. The rate of energy release, hydrogen generation, and cladding oxidatior from the metal/water reaction shall be calculated using the Baker-Just equation (Baker, L., Just, L.C. "Studies of Metal Water Reactions at High Temperatures, III. Experimental and Theoretical Studies of the Zirconium-Water Reaction," ANL-6548, page 7, May 1962). The reaction shall be assumed not to be stean limited. For rods whose cladding is calculated to rupture during the LOCA, the inside of the cladding shal also be assumed to react after the rupture. The calculation of the reaction rate on the inside of the cladding shall also follow the Baker-Just equation, starting at the time when the cladding is calculated to rupture and extending around the cladding inner circumference and axially no less than 1.5 inches each way from the location of the rupture, with the reaction assumed not to be steam limited.

6. Reactor Internals Heat Transfer. Heat transfer from piping, vessel walls, and non-fuel internal hardware shall be taken into account.

7.Pressurized Water Reactor Primary-to-Secondary Heat Transfer. Heat transferred between primary and secondary systems through heat exchangers (steam generators) shall be taken into account. (Not applicable to Boiling Water Reactors.)

\section{B. SWELLING AND RUPTURE OF THE CLADDING AND FUEL ROD THERMAL PARAMETERS}

Each evaluation model shall include a provision for predicting cladding swelling and rupture from consideration of the axial temperature distribution of the cladding and from the difference in pressure between the inside and outside of the cladding, both as functions of time. To be acceptable the swelling and 
rupture calculations shall be based on applicable data in such a way that the degree of swelling and incidence of rupture are not underestimated. The degree of swelling and rupture shall be taken into account in calculations of gap conductance, cladding oxidation and embrittlement, and hydrogen generation.

The calculations of fuel and cladding temperatures as a function of time shall use values for gap conductance and other thermal parameters as functions of temperature and other applicable timedependent variables. The gap conductance shall be varied in accordance with changes in gap dimensions and any other applicable variables.

\section{BLOWDOWN PHENOMENA}

\section{Break Characteristics and Flow}

a. In analyses of hypothetical loss-of-coolant accidents, a spectrum of possible pipe breaks shall be considered. This spectrum shall include instantaneous double-ended breaks ranging in cross-sectional area up to and including that of the largest pipe in the primary coolant system. The analysis shall also include the effects of longitudinal splits in the largest pipes, with the split area equal to the cross-sectional area of the pipe.

b. Discharge Model. For all times after the discharging fluid has been calculated to be two-phase in composition, the discharge rate shall be calculated by use of the Moody model (F. J. Moody, "Maximum Flow Rate of a Single Component, Two-Phase Mixture," Journal of Heat Transfer, Transactions of the American Society of Mechanical Engineers, 87, No. 1, February 1965). The calculation shall be conducted with at least three values of a discharge coefficient applied to the postulated break area, these values spanning the range from 0.6 to 1.0. If the results indicate that the maximum clad temperature for the hypothetical accident is to be found at an even lower value of the discharge coefficient, the range of discharge coefficients shall be extended until the maximum clad temperature calculated by this variation has been achieved.

c. End of Blowdown. (Applies Only to Pressurized Water Reactors.) For postulated cold leg breaks, all emergency cooling water injected into the inlet lines or the reactor vessel during the bypass period shall in the calculations be subtracted from the reactor vessel calculated inventory. This may be executed in the calculation during the bypass period, or as an alternative the amount of emergency core cooling water calculated to be injected during the bypass period may be subtracted later in the calculation from the water remaining in the inlet lines, downcomer, and reactor vessel lower plenum after the bypass period. This bypassing shall end in the calculation at a time designated as the "end of bypass," after which the expulsion or entrainment mechanisms responsible for the bypassing are calculated not to be effective. The end-of-bypass definition used in the calculation shall be justified by a suitable combination of analysis and experimental data. Acceptable methods for defining "end of bypass" include, but are not limited to, the following: (1) Prediction of the blowdown calculation of downward flow in the downcomer for the remainder of the blowdown period; (2) Prediction of a threshold for droplet entrainment in the upward velocity, using local fluid conditions and a conservative critical Weber number.

d. Noding Near the Break and the ECCS Injection Points. The noding in the vicinity of and including the broken or split sections of pipe and the points of ECCS injection shall be chosen to permit a reliable analysis of the thermodynamic history in these regions during blowdown.

2. Frictional Pressure Drops. The frictional losses in pipes and other components including the reactor core shall be calculated using models that include realistic variation of friction factor with Reynolds number, and realistic two-phase friction multipliers that have been adequately verified by comparison with experimental data, or models that prove at least equally conservative with respect to maximum clad temperature calculated during the hypothetical accident. The modified Baroczy correlation (Baroczy, C. J., "A Systematic Correlation for Two-Phase Pressure Drop," Chem. Enging. Prog. Symp. Series, No. 64, Vol. 62, 1965) or a combination of the Thom correlation (Thom, J. R. S., "Prediction of Pressure Drop During Forced Circulation Boiling of Water," Int. J. of Heat \& Mass Transfer, 7, 709-724, 1964) for pressures equal to or greater than 250 psia and the Martinelli-Nelson correlation (Martinelli, R. C., Nelson, D. B., "Prediction of Pressure Drop During Forced Circulation Boiling of Water," Transactions of ASME, $695-702,1948)$ for pressures lower than 250 psia is acceptable as a basis for calculating realistic two-phase friction multipliers.

3. Momentum Equation. The following effects shall be taken into account in the conservation of momentum equation: (1) temporal change of momentum, (2) momentum convection, (3) area change momentum flux, (4) momentum change due to compressibility, (5) pressure loss resulting from wall friction, (6) pressure loss resulting from area change, and (7) gravitational acceleration. Any omission of one or more of these terms under stated circumstances shall be justified by comparative analyses or by experimental data.

\section{Critical Heat Flux}

a. Correlations developed from appropriate steady-state and transient-state experimental data are acceptable for use in predicting the critical heat flux (CHF) during LOCA transients. The computer programs in which these correlations are used shall contain suitable checks to assure that the physical parameters are within the range of parameters specified for use of the correlations by their respective authors. 
b. Steady-state CHF correlations acceptable for use in LOCA transients include, but are not limited to, the following:

(1) W 3. L. S. Tong, "Prediction of Departure from Nucleate Boiling for an Axially Non-uniform Heat Flux Distribution," Journal of Nuclear Energy, Vol. 21, 241-248, 1967.

(2) $B \& W-2$. J. S. Gellerstedt, R. A. Lee, W. J. Oberjohn, R. H. Wilson, L. J. Stanek, "Correlation of Critical Heat Flux in a Bundle Cooled by Pressurized Water," Two-Phase Flow and Heat Transfer in Rod Bundles, ASME, New York, 1969.

(3) Hench-Levy. J. M. Healzer, J. E. Hench, E. Janssen, S. Levy, "Design Basis for Critical Heat Flux Condition in Boiling Water Reactors," APED-5186, GE Company Private report, July 1966.

(4) Macbeth. R. V. Macbeth, "An Appraisal of Forced Convection Burnout Data," Proceedings of the Institute of Mechanical Engineers, 1965-1966.

(5) Barnett. P. G. Barnett, "A Correlation of Burnout Data for Uniformly Heated Annuli and Its Uses for Predicting Burnout in Uniformly Heated Rod Bundles," AEEW-R 463, 1966.

(6) Hughes. E. D. Hughes, "A Correlation of Rod Bundle Critical Heat Flux for Water in the Pressure Range 150 to 725 psia,: IN-1412, Idaho Nuclear Corporation, July 1970.

c. Correlations of appropriate transient CHF data may be accepted for use in LOCA transient analyses if comparisons between the data and the correlations are provided to demonstrate that the correlations predict values of CHF which allow for uncertainty in the experimental data throughout the range of parameters for which the correlations are to be used. Where appropriate, the comparisons shall use statistical uncertainty analysis of the data to demonstrate the conservatism of the transient correlation.

d. Transient CHF correlations acceptable for use in LOCA transients include, but are not limited to, the following:

(1) GE Transient CHF. B. C. Slifer, J. E. Hench, "Loss-of-Coolant Accident and Emergency Core Cooling Models for General Electric Boiling Water Reactors," NEDO-10329, General Electric Company, Equation C-32, April 1971.

e. After CHF is first predicted at an axial fuel rod location during blowdown, the calculation shall not use nucleate boiling heat transfer correlations at that location subsequently during the blowdown even if the calculated local fluid and surface conditions would apparently justify the reestablishment of nucleate boiling. Heat transfer assumptions characteristic of return to nucleate boiling (rewetting) shall be permitted when justified by the calculated local fluid and surface conditions during the reflood portion of a LOCA.

\section{Post-CHF Heat Transfer Correlations}

a. Correlations of heat transfer from the fuel cladding to the surrounding fluid in the post-CHF regimes of transition and film boiling shall be compared to applicable steady-state and transient-state data using statistical correlation and uncertainty analyses. Such comparison shall demonstrate that the correlations predict values of heat transfer coefficient equal to or less than the mean value of the applicable experimental heat transfer data throughout the range of parameters for which the correlations are to be used. The comparisons shall quantify the relation of the correlations to the statistical uncertainty of the applicable data.

b. The Groeneveld flow film boiling correlation (Equation 5.7 of D. C. Groeneveld, "An Investigation of Heat Transfer in the Liquid Deficient Regime," AECL-3281, revised December 1969), the Dougall-Rohsenow flow film boiling correlation (R. S. Dougall and W. M. Rohsenow, "Film Boiling on the Inside of Vertical Tubes with Upward Flow of the Fluid at Low Qualities," MIT Report Number 9079-26, Cambridge, Massachusetts, September 1963), and the Westinghouse correlation of steady-state transition boiling ("Proprietary Redirect/Rebuttal Testimony of Westinghouse Electric Corporation," U.S.A.E.C. Docket RM-50-1, page 25-1, October 26, 1972) are acceptable for use in the post-CHF boiling regimes. In addition the transition boiling correlation of McDonough, Milich, and King (J. B. McDonough, W. Milich, E. C. King, "Partial Film Boiling with Water at 2000 psig in a Round Vertical Tube," MSA Research Corp., Technical Report 62 (NP-6976), (1958) is suitable for use between nucleate and film boiling. Use of all these correlations shall be restricted as follows:

(1) The Groeneveld correlation shall not be used in the region near its low-pressure singularity,

(2) the first term (nucleate) of the Westinghouse correlation and the entire McDonough, Milich, and King correlation shall not be used during the blowdown after the temperature difference between the clad and the saturated fluid first exceeds $300^{\circ} \mathrm{F}$,

(3) transition boiling heat transfer shall not be reapplied for the remainder of the LOCA blowdown, even if the clad superheat returns below $300^{\circ} \mathrm{F}$, except for the reflood portion of the LOCA when justified by the calculated local fluid and surface conditions.

6. Pump Modeling. The characteristics of rotating primary system pumps (axial flow, turbine, or centrifugal) shall be derived from a dynamic model that includes momentum transfer between the fluid and the rotating member, with variable pump speed as a function of time. The pump model resistance used for analysis should be justified. The pump model for the two-phase region shall be verified by applicable two-phase pump performance data. For BWR's after saturation is calculated at the pump suction, the pump head may be assumed to vary linearly with quality, going to zero for one percent quality at the pump suction, so long as the analysis shows that core flow stops before the quality at pump suction reaches one percent.

7. Core Flow Distribution During Blowdown. (Applies only to pressurized water reactors.)

a. The flow rate through the hot region of the core during blowdown shall be calculated as a function of time. For the purpose of these calculations the hot region chosen shall not be greater than the size of one 
fuel assembly. Calculations of average flow and flow in the hot region shall take into account cross flow between regions and any flow blockage calculated to occur during blowdown as a result of cladding swelling or rupture. The calculated flow shall be smoothed to eliminate any calculated rapid oscillations (period less than 0.1 seconds).

b. A method shall be specified for determining the enthalpy to be used as input data to the hot channel heatup analysis from quantities calculated in the blowdown analysis, consistent with the flow distribution calculations.

\section{POST-BLOWDOWN PHENOMENA; HEAT REMOVAL BY THE ECCS}

1. Single Failure Criterion. An analysis of possible failure modes of ECCS equipment and of their effects on ECCS performance must be made. In carrying out the accident evaluation the combination of ECCS subsystems assumed to be operative shall be those available after the most damaging single failure of ECCS equipment has taken place.

2. Containment Pressure. The containment pressure used for evaluating cooling effectiveness during reflood and spray cooling shall not exceed a pressure calculated conservatively for this purpose. The calculation shall include the effects of operation of all installed pressure-reducing systems and processes.

3. Calculation of Reflood Rate for Pressurized Water Reactors. The refilling of the reactor vessel and the time and rate of reflooding of the core shall be calculated by an acceptable model that takes into consideration the thermal and hydraulic characteristics of the core and of the reactor system. The primary system coolant pumps shall be assumed to have locked impellers if this assumption leads to the maximum calculated cladding temperature; otherwise the pump rotor shall be assumed to be running free. The ratio of the total fluid flow at the core exit plane to the total liquid flow at the core inlet plane (carryover fraction) shall be used to determine the core exit flow and shall be determined in accordance with applicable experimental data (for example, "PWR FLECHT (Füll Length Emergency Cooling Heat Transfer) Final Report," Westinghouse Report WCAP-7665; April 1971; "PWR Full Length Emergency Cooling Heat Transfer (FLECHT) Group I Test Report," Westinghouse Report WCAP-7435, January 1970; "PWR FLECHT (Full Length Emergency Cooling Heat Transfer) Group II Test Report," Westinghouse Report WCAP-7544, September 1970; "PWR FLECHT Final Report Supplement," Westinghouse Report WCAP-7931, October 1972).

The effects on reflooding rate of the compressed gas in the accumulator which is discharged following accumulator water discharge shall also be taken into account.

4. Steam Interaction with Emergency Core Cooling Water in Pressurized Water Reactors. The thermal-hydraulic interaction between steam and all emergency core cooling water shall be taken into account in calculating the core reflooding rate. During refill and reflood, the calculated steam flow in unbroken reactor coolant pipes shall be taken to be zero during the time that accumulators are discharging water into those pipes unless experimental evidence is available regarding the realistic thermal-hydraulic interaction between the steam and the liquid. In this case, the experimental data may be used to support an alternate assumption.

5. Refill and Reflood Heat Transfer for Pressurized Water Reactors. For reflood rates of one inch per second or higher, reflood heat transfer coefficients shall be based on. applicable experimental data for unblocked cores including FLECHT results ("PWR FLECHT (Full Length Emergency Cooling Heat Transfer) Final Report," Westinghouse Report WCAP-7665, April 1971). The use of a correlation derived from FLECHT data shall be demonstrated to be conservative for the transient to which it is applied; presently available FLECHT heat transfer correlations ("PWR Full Length Emergency Cooling Heat Transfer (FLECHT) Group I Test Report," Westinghouse Report WCAP-7544, September 1970; "PWR FLECHT Final Report Supplement," Westinghouse Report WCAP-7931, October 1972) are not acceptable. New correlations or modifications to the FLECHT heat transfer correlations are acceptable only after they are demonstrated to be conservative, by comparison with FLECHT data, for a range of parameters consistent with the transient to which they are applied.

During refill and during reflood when reflood rates are less than one inch per second, heat transfer calculations shall be based on the assumption that cooling is only by steam, and shall take into account any flow blockage calculated to occur as a result of cladding swelling or rupture as such blockage might affect both local steam flow and heat transfer.

6. Convective Heat Transfer Coefficients for Boiling Water Reactor Fuel Rods Under Spray Cooling. Following the blowdown period, convective heat transfer shall be calculated using coefficients based on appropriate experimental data. For reactors with jet pumps and having fuel rods in a $7 \times 7$ fuel assembly array, the following convective coefficients are acceptable:

a. During the period following lower plenum flashing but prior to the core spray reaching rated flow, a convective heat transfer coefficient of zero shall be applied to all fuel rods.

b. During the period after core spray reaches rated flow but prior to reflooding, convective heat transfer coefficients of $3.0,3.5,1.5$, and $1.5 \mathrm{Btu}-\mathrm{hr}^{-1}-\mathrm{ft}^{-2}-{ }^{\circ} \mathrm{F}^{-1}$ shall be applied to the fuel rods in the outer corners, outer row, next to outer row, and to those remaining in the interior, respectively, of the assembly.

c. After the two-phase reflooding fluid reaches the level under consideration, a convective heat transfer coefficient of $25 \mathrm{Btu}^{-\mathrm{hr}^{-1}} \cdot \mathrm{ft}^{-2}{ }^{\circ} \mathrm{F}^{-1}$ shall be applied to all fuel rods.

7. The Boiling Water Reactor Channel Box Under Spray Cooling. Following the blowdown period, heat transfer from, and wetting of, the channel box shall be based on appropriate experimental data. For reactors with jet pumps and fuel rods in a $7 \times 7$ fuel assembly array, the following heat transfer coefficients and wetting time correlation are acceptable.

a. During the period after lower plenum flashing, but prior to core spray reaching rated flow, a convective coefficient of zero shall be applied to the fuel assembly channel box.

b. During the period after core spray reaches rated flow, but prior to wetting of the channel, a convective heat transfer coefficient of $5 \mathrm{Btu}-\mathrm{hr}^{-1}-\mathrm{ft}^{-2} \cdot{ }^{\circ} \mathrm{F}^{-1}$ shall be applied to both sides of the channel box.

c. Wetting of the channel box shall be assumed to occur 60 seconds after the time determined using the 
correlation based on the Yamanouchi analysis ("Loss-of-Coolant Accident and Emergency Core Cooling Models for General Electric Boiling Water Reactors," General Electric Company Report NEDO-10329, April 1971).

\section{REQUIRED DOCUMENTATION}

1.a. A description of each evaluation model shall be furnished. The description shall be sufficiently complete to permit technical review of the analytical approach including the equations used, their approximations in difference form, the assumptions made, and the values of all parameters or the procedure for their selection, as for example, in accordance with a specified physical law or empirical correlation.

b. The description shall be sufficiently detailed and specific to require significant changes in the evaluation model to be specified in amendments of the description. For this purpose, a significant change is a change that would result in a calculated fuel cladding temperature different by more than $20^{\circ} \mathrm{F}$ from the temperature calculated (as a function of time) for a postulated LOCA using the last previously accepted model.

c. A complete listing of each computer program, in the same form as used in the evaluation model, shall be furnished to the Atomic Energy Commission.

2. For each computer program, solution convergence shall be demonstrated by studies of system modeling or noding and calculational time steps.

3. Appropriate sensitivity studies shall be performed for each evaluation model, to evaluate the effect on the calculated results of variations in noding, phenomena assumed in the calculation to predominate, including pump operation or locking, and values of parameters over their applicable ranges. For items to which results are shown to be sensitive, the choices made shall be justified.

4. To the extent practicable, predictions of the evaluation model, or portions thereof, shall be compared with applicable experimental information.

5. General Standards for Acceptability-Elements of evaluation models reviewed will include technical adequacy of the calculational methods, including compliance with required features of Section I of this Appendix $\mathrm{K}$ and provision of a level of safety and margin of conservatism comparable to other acceptable evaluation models, taking into account significant differences in the reactors to which they apply. 


\section{REFERENCES}

AEC Commissioners,
1974

AEC/ECCS, 1967

AEC/OOE, 1972

AEC/OOE, 1974a

$\mathrm{AEC} / \mathrm{OOE}, 1974 \mathrm{~b}$

AEC/REG, 1973

AEC, WASH-740, 1957

AEC, WASH-1139, 1974

AEC, WASH-1209, 1973

AEC, WASH-1250, 1973

AEC, WASH-1258, 1973

AEC, WASH-1270, 1973
U.S. Atomic Energy Commissioners

(Ray, Larson, Doub, Kriegsman, and Anders), 1974, "Opinion of the Commission-In the Matter of Rulemaking Hearing-Acceptance Criteria for Emergency Core Cooling Systems for Light-Water-Cooled Nuclear Power Reactors," in Regulatory Adjudication Issuances, RAI-73-12.

U. S. Atomic Energy Commission, 1967, W. K. Ergen et al., "Emergency Core Cooling: Report of the Advisory Task Force Committee on Power Reactor Emergency Cooling."

U.S. Atomic Energy Commission Office of Operations Evaluation, 1972, "Evaluation of Incidents of Primary Coolant Release from Operating Boiling Water Reactors."

U. S. Atomic Energy Commission Office of Operations Evaluation, 1974, Report OOE-ES-002, "Diesel Generator Operating Experience at Nuclear Power Plants."

U. S. Atomic Energy Commission Office of Operations Evaluation, 1974, Report OOE-OS-001, "Summary of Abnormal Occurrences Reported to the AEC during 1973."

U.S. Atomic Energy Commission Regulatory Staff, 1973, "Final Environmental Statement Concerning Proposed Rulemaking Action: Acceptance Criteria for Emergency Core Cooling Systems for Light-Water-Cooled Nuclear Power Reactors."

U.S. Atomic Energy Commission, 1957, Report WASH-740, "Theoretical Possibilities and Consequences of Major Accidents in Large Nuclear Plants."

U. S. Atomic Energy Commission, 1974, Report WASH-1139, "Nuclear Power Growth, 1974-2000."

U.S. Atomic Energy Commission, 1973, Report WASH-1209, "The Potential Radiological Implications of Nuclear Facilities in the Upper Mississippi River Basin in the Year 2000.'

U.S. Atomic Energy Commission, 1973, Report WASH-1250, "The Safety of Nuclear Power Reactors (LightWater-Cooled) and Related Facilities."

U.S. Atomic Energy Commission, 1973, Report WASH-1258, "Final Environmental Statement Concerning Proposed Rule-Making Action: Numeral Guides for Design Objectives and Limiting Conditions for Operation to Meet the Criterion 'As Low as Practicable' for Radioactive Material in Light-Water-Cooled Nuclear Power Reactor Effluents."

U.S. Atomic Energy Commission, 1973 Report WASH-1270, "Technical Report on Anticipated Transients Without SCRAM for Water-Cooled Power Reactors."
Conard, 1975

AEC, WASH-1285, 1974

AEC, WASH-1288, 1974

AEC, WASH-1400, 1974

Aerojet, 1974

ANS, 1973

Beck and Watson, 1973

Bennett, 1973

Blake et al., 1973

Bond, 1975

Brockett et al., 1972

Code of Federal

Regulations

Codek et al . , 1971

Cole, 1972

Coplen et al., 1974
U.S. Atomic Energy Commission, Advisory Committee on Reactor Safety, 1974, Report WASH-1285, "Report on the Integrity of Reactor Vessels for Light-Water Power Reactors."

U. S. Atomic Energy Commission, 1974, Preliminary Report WASH-1288, "Evaluation of Nuclear Energy Centers."

U.S. Atomic Energy Commission, 1974, Draft of Report WASH-1400, "Reactor Safety Study: An Assessment of Accident Risks in U.S. Commercial Nuclear Power Plants."

Aerojet Nuclear Company, 1974 , "LOFT Integral Test System Design Basis Report," ANC, Idaho Falls, ID (unpublished).

American Nuclear Society, 1973, Symposium on Power Plant Dynamics, Control, and Testing (Amer. Nucl. Society, Knoxville, TN).

Beck, R. L., and M. B. Watson, 1973, "Safety Analysis of an Underground Nuclear Power Plant with an Inoperable ECCS," Draft Report ATR-73 (72778)-3, Aerospace Corp., Los Angeles (unpublished).

Bennett, D.J., 1973, The Elements of Nuclear Power (Wiley, New York).

Blake, A., V. N. Korpenko, E.W. McDawley, and C.E. Walter, 1973, "A Concept for Underground Siting of Nuclear Power Reactors," Lawrence Livermore Laboratory Report UCRL-51408, Livermore, California (unpublished).

Bond, V., 1975, private communication, Brookhaven National Laboratory.

Brockett, G.E., R.W. Griebe, R. W. Shumway, and J. O. Zane, 1972, "Loss of Coolant: Control of Consequences by Emergency Control Cooling," in Proceedings of the International Conference on Nuclear Solutions to World Energy Problems, Joint American Nuclear Society/Atomic Industrial Forum Conference, 13-17 November 1972, Washington, D. C.

Code of Federal Regulations, 10 CFR 55, Appendix A. Published by the Division of the Federal Register, the National Archives.

Codek, F. F., D. P. Domenicis, and R. H. Leyse, 1971, "PWR-FLECHT Final Report," Report WCAP-7665, Westinghouse Electric Corp., Pittsburgh, PA (unpublished).

Cole, R., 1972, Final Report: Inhalation of Radioiodine from Fallout: Hazards and Countermeasures (Environmental Science Associates, Burlingame, CA).

Conard, R.A., 1975, Report BNL-50424, Brookhaven National Laboratory, Upton, N.Y. (to be published).

Coplen, H. L., L. J. Ybarrondo, R. W. Barber, and R.E. Swanson, 
Crank, 1956

Crawley and McCreath, 1972

DeWiest, 1969

Doniach, 1963

Draft WASH-1400

Duncan and Leonard, 1971

Edwards and

O’Brien, 1970

EPA, 1973

Epler and Oakes, 1973

Finlayson, 1975

Gambill, 1973

Gamer, 1973

General Electric

Co. , 1970

Gifford, 1967

Glasstone and Sesonske, 1963
1974, "The LOFT Integral Test Facility and Program," Ninth Intersociety Energy Conversion Engineering Conference, San Francisco (unpublished).

Cottrell, W. B。, 1974, Nuclear Safety $15,52$.

Crank, J., 1956, The Mathematics of Diffusion (Clarendon Press, Oxford).

Crawley, J.H. , and D.R. McCreath, 1972, "The Underground: An Alternative Site for Nuclear Power Reactors," Report UEC-UNP-720207, United Engineers Co. and Acres American, Inc., Philadelphia, PA (unpublished).

DeWiest, R. J. M. , 1969, Editor, Flow through Porous Media (Academic Press, New York).

Doniach, I., 1963, Health Physics 9, 1357

Sec AEC, WASH-1400, 1974.

Duncan, J.D., and J.E. Leonard, 1971, "Emergency Cooling in BoilingWater Reactors under Simulated Lossof-Coolant Conditions (BWR-FLECHT Final Report)," Report GEAP-13197, General Electric Company, San Jose, CA (unpublished).

Edwards, A.R., and T.P. O'Brien, 1970 , J. Br. Nucl. Energy Soc. 9, 125.

Green and Lane, 1963

Healy, 1966

Hempelman, 1968

Hempelman et al., 1975

Hobson and

Rittenhouse, 1972

U. S. Environmental Protection Agency, 1973, "Environmental Analysis of the Uranium Fuel Cycle, Part II: Nuclear Power Reactors," Report EPA-520/974-003-C.

Epler, E.P., and L. C. Oakes, 1973, Nuclear Safety 14, 95.

Finlayson, F. C., 1975, "Emergency Core Cooling Systems for Light Water Reactors," Environmental Quality Laboratory, California Institute of Technology, Pasadena, Calif. (to be published).

Gambill, W.R., 1973, "Estimate of Effect of Localized Flow Blockages on PWR Clad Temperatures During Reflood," in Proceedings of Topical Meeting on Water Reactor Safety, Salt Lake City, Utah, March 26-28, 1973, published as US AEC/TIC Report CONF-7303040, pp. 221-240.

Gamer, R. W. , 1973, "Comparative Analyses of Standard Problems-Standard Problem (Straight Pipe Depressurization Experiments)," Interim Report 1-212, 74-5.1, Aerojet Nuclear Company, Idaho Falls, ID (unpublished).

"Core Study Cooling Systems for the General Electric 1969 BWR Standard Plants," Rept. NEDO-10183 General Electric Co., San Jose, Calif. (unpublished)

Gifford, F.A., 1967, J. Appl. Meteorol. 6, 644.

Glasstone, S. , and A. Sesonske, 1963, Nuclear Reactor Engineering (Van Nostrand, New York).

ICRP, 1959

Jansen and Stepnowski, 1973

Klassovskii, 1967

Kouts, 1974

Lomanik, 1970

Mochizuki et al., 1963

Morrison et al. , 1971
Green, H. L., and W.R. Lane, 1964 Particulate Clouds: Dusts, Smokes, and Mists (Belfast Universities Press, Belfast, Ireland).

Healy, J.W., 1966, "Nuclear Reactor Accident-Damage Assessment, Draft," Los Alsmos Scientific Laboratory, Los Alamos, New Mexico (unpublished).

Hempelman, L. H. , 1968, Science 160 , 159.

Hempelman, L. H., W. J. Hall, M. Phillips, R. A. Cooper, and W.R. Ames, 1975, "Neoplasms in Persons Treated with X-Rays in Infancy, Fourth Survey" (University of Rochester, Rochester, New York, to be published).

Hewitt, G. F., 1972, in Proceedings of the International Symposium on Two-Phase Systems, edited by G.

Hetsroni, S. Sideman, and J.P. Hartnett, published as Volume 6 of Progress in Heat and Mass Transfer (Pergamon, Oxford), p. 295.

Hobson, D. O. , and P. L. Rittenhouse, 1972, "Embrittlement of Zircaloyclad Fuel Rods by Steam During LOCA Transients," Report ORNL-4758, Oak Ridge National Laboratory, Oak Ridge, TN (unpublished)

International Commission on Radiological Protection, 1959, "Radiation Protection: Recommendations of the ICRP," ICRP Report No. 2 (Pergamon, London).

Ilin et al., $1972 \quad$ Ilin, L. A., G. V. Archangelskaya, Y. O. Kostentinov, and I. A. Llikhtarev, 1972, "Radioactive Iodine in the Problem of Radiation Safety," Atomizdat, Moscow (available from AEC, Washington, as AEC-tr-7536). Jansen, G. , and D. D. Stepnowski 1973, Nuclear Technology 17, 85.

Klassovskii, Iu. A., 1967, "Dependency of Irradiation Effect on Determination of Dose in Thyroid Histological Structures," Radiatsionnaia Endokinologiia, Akademiia Meditsinskikh Nauk SSSR, p. 40.

Kouts, H. J. C. , 1974, Nuclear Safety $15,127$.

Lomanik, T. F., 1970, "Earthquakes and Nuclear Power Plant Design," Report ORNL-NSIC-28, Oak Ridge National Laboratory, Oak Ridge, TN (unpublished).

Mochizuki, Y., R. Mowafy, and B. Pasternack, 1963, Health Physics $9,1299$.

Morrison, D. L. , R. L. Ritzman, R. O. Wooton, J. M. Genco, R.H. Barnes, J. M. Fackelmann, and W. A. Carbiener, 1971, "An Evaluation of the Applicability of Existing Data to the Analytical Description of a Nuclear Reactor Accident-Core Meltdown Evaluation," Report BMI-1910, Battelle Memorial Institute, Columbus, Ohio (unpublished). 
NAS, 1972

NCI, 1972

NRC, $1975 \mathrm{a}$

NRC, $1975 b$

Perry et al., 1973

Robertson and

Barraclough, 1973

Saidl and Rakova, 1966

Scatena, 1972
National Academy of Sciences, 1972, "The Effects on Populations of Exposures to Low Levels of Ionizing Radiation" (popularity known as the BEIR Report).

National Cancer Institute, 1972, End Results in Cancer, Report No. 4 Copy of briefing materials presented by the Nuclear Regulatory Commission at a meeting in Washington, March 17 . 1975 to a subgroup of the APS study group on light-water reactor safety.

Letter dated March 27, 1975 from

I. B. Wall of the Nuclear Regulatory Commission to $F$. von Hippel.

Perry, A.M. , F.C. Maienschein, and D. R. Vondy, "Fission-Product Afterheat: A Review of Experiments Pertinent to the Thermal-Neutron Fission of U-235," Report ORNL-TM4197, Oak Ridge National Laboratory, Oak Ridge, TN (unpublished).

Robertson, J. B., and J.T.

Barraclough, 1973, Underground

Waste Management and Artificial Recharge 1, 291.

Saidl, J., and J. Rakova, 1966, Collect. Czech, Chem. Commun。31, 871.

Scatena, G. J. , 1972, "Fuel Cladding Embrittlement During a Loss-ofCoolant Accident," Report NEDO-10674, General Electric Company, San Jose, CA (unpublished).
Shure, 1972

Slade, 1968

Turner, 1970

UNSCEAR， 1972

Watson et al. 1972

Yadigaroglu and Andersen, 1974

Ybarrondo et al. 1975

Zuber, 1974
Shure, K., 1972, "U-235 Fission Product Decay Energy--1972 Reevaluation," Report WAPD-TM-1119, Bettis Atomic Power Laboratory, Pittsburgh, PA (unpublished).

Slade, D.H. , 1968, editor, Meteorology and Atomic Energy, U. S. Atomic Energy Commission, Washington, D。C.

Turner, D. B. , 1970, Workbook of Atmospheri c Dispersion Estimates, U.S. Environmental Protection Agency, Washington, D。C.

United National Scientific Committee on the Effects of Atomic Radiation 1972, Ionizing Radiation: Levels and Effects (United Nations, New York).

Watson, H. B., W.A. Kammer, N. P. Langley, L. A. Selzer, and R. L. Beck, 1972, "Underground Nuclear Power Plant Siting," Report EQL-6, Environmental Quality Laboratory Number 6, Caltech.

Yadigaroglu, G., and S. O. Andersen, 1974, Nuclear Safety 15, 651.

Ybarrondo, L. J., S。 Fabic, P. Griffith, and G. D. McPherson, 1975, J. Am. Soc. Mech. Eng。 74, WA/HT 53.

Zuber, N. , 1974, "On the VolumeAveraged Conservatism Equations," U. S. Atomic Energy Commission, Division of Reactor Safety Research, Washington, D.C. , June 14, 1974 (unpublished). 


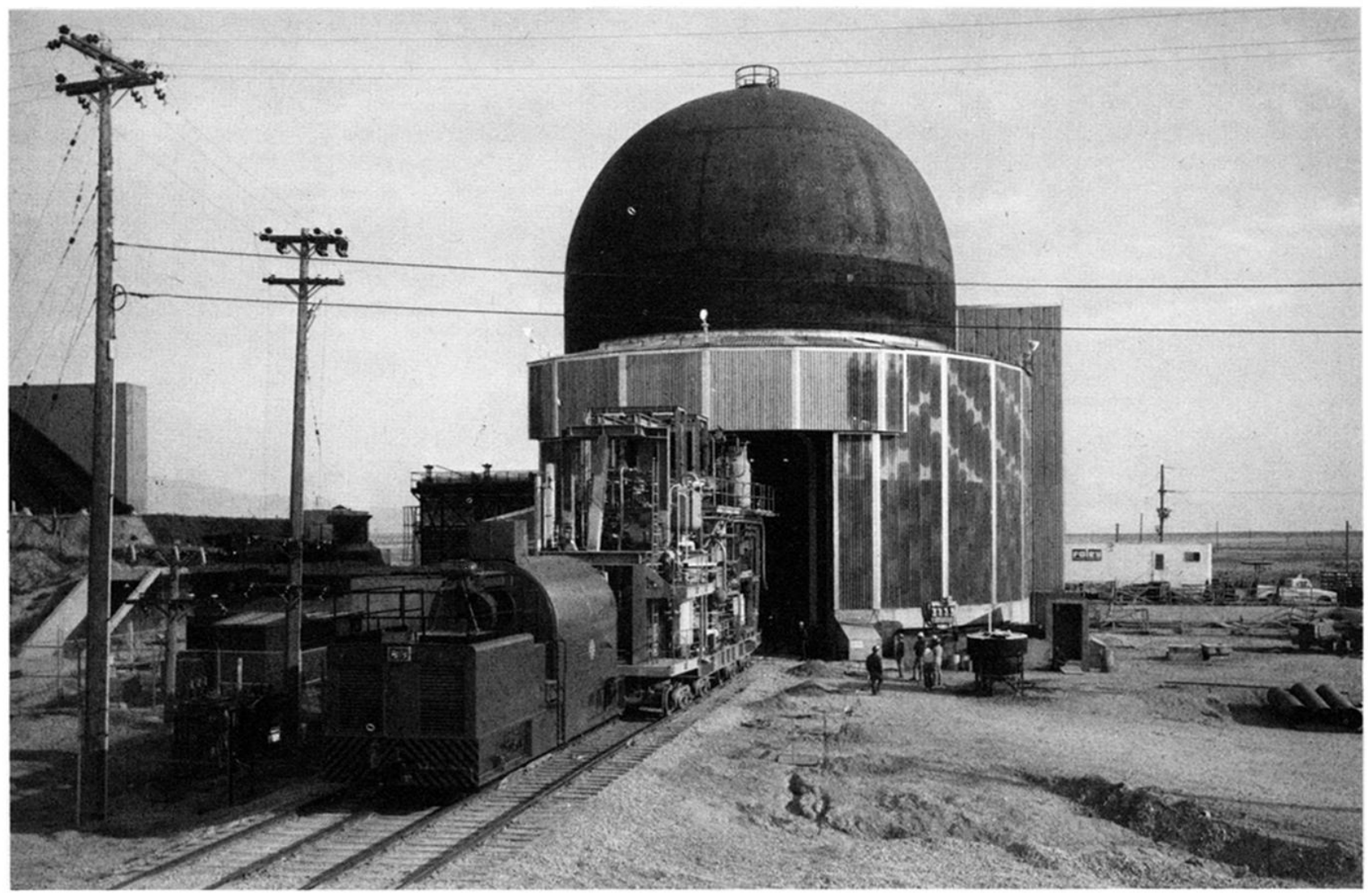

(a)

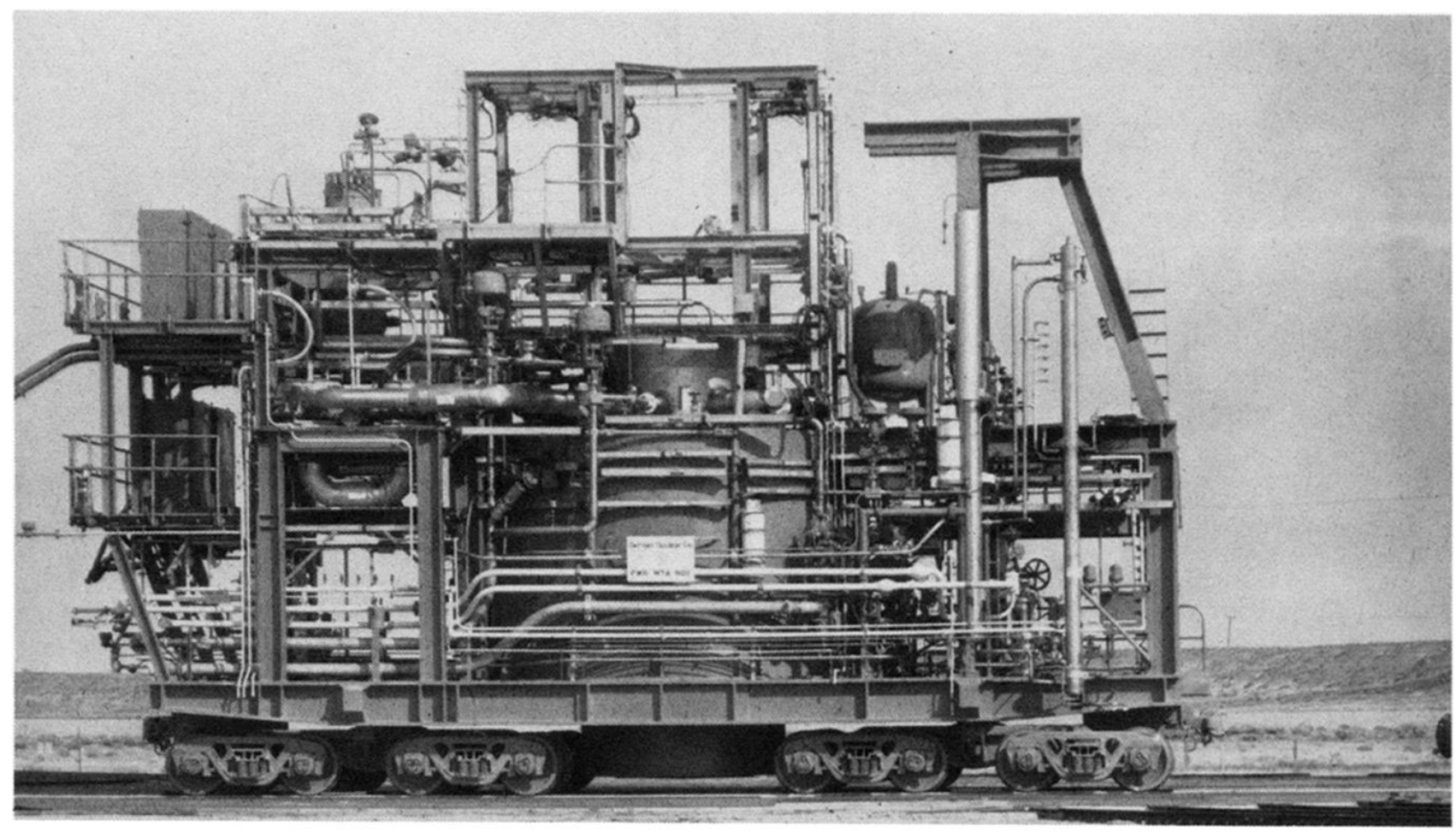

(b)

FIG. 11. (a) LOFT mobile test assembly entering the test chamber. (b) Side view of LOFT mobile test assembly. 


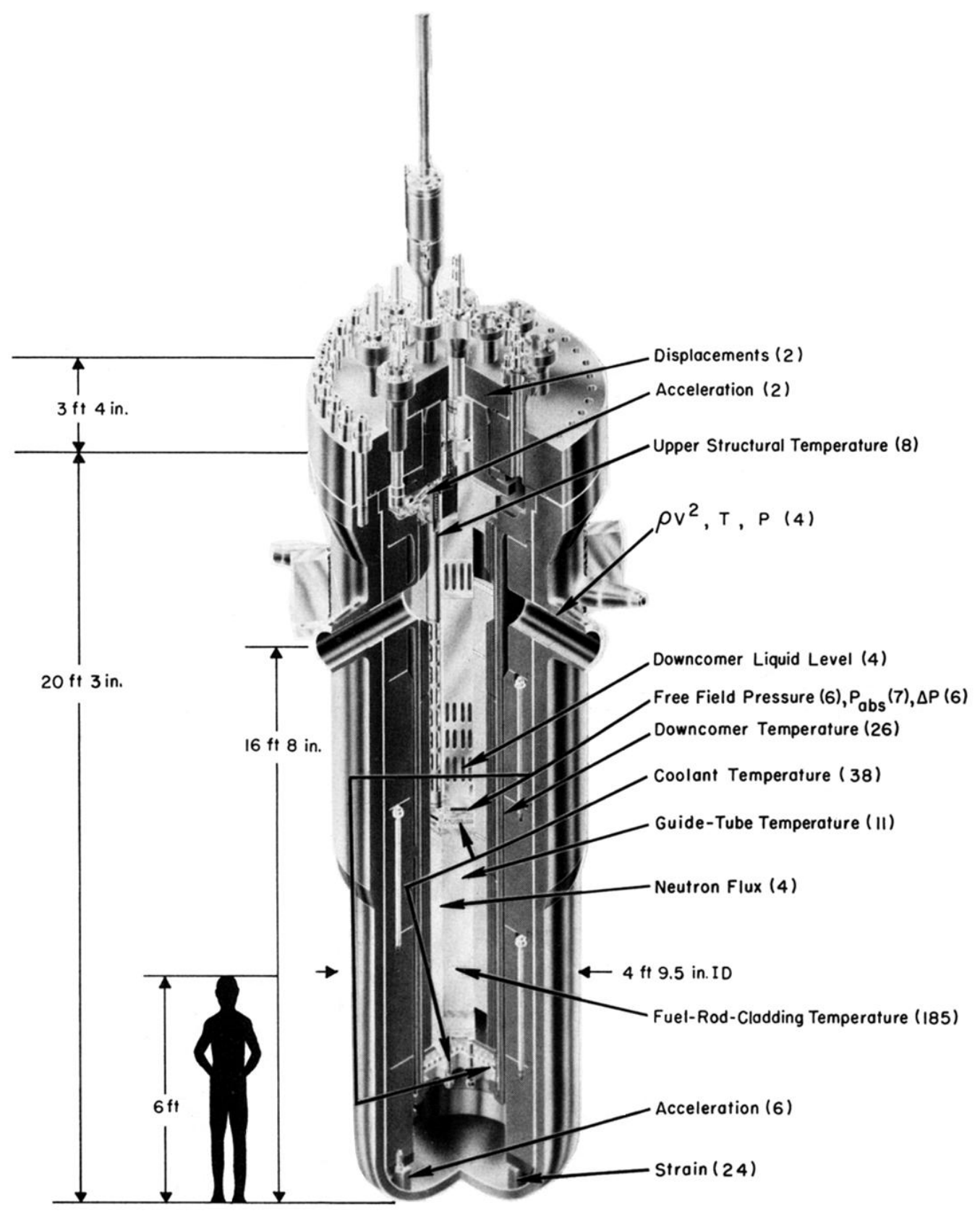

FIG. 12. Schematic of LOFT reactor vessel with associated experimental instrumentation. 


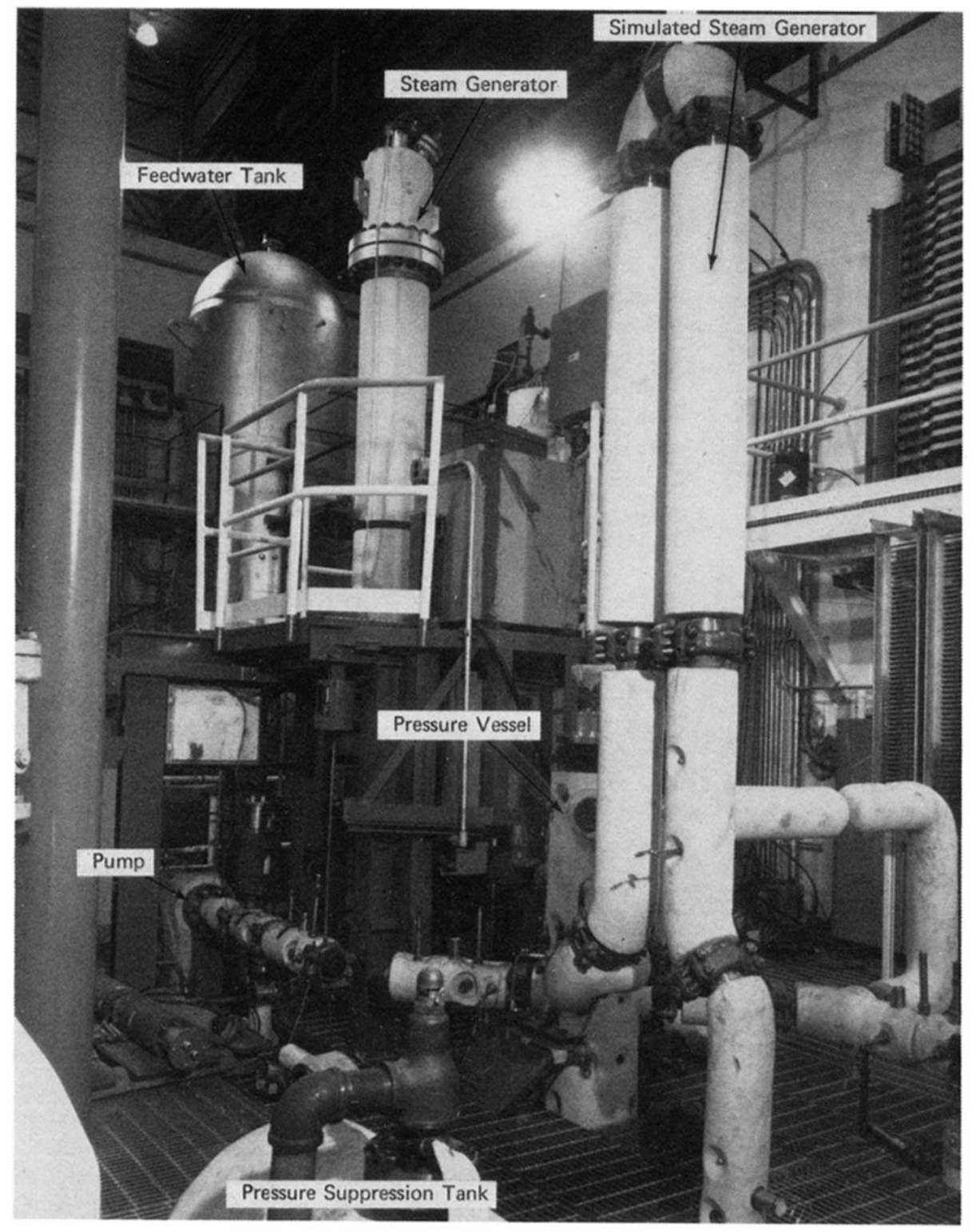

FIG. 14. Thermal reactor safety program: Semiscale Mod-1. 